\author{
Monograph \\ urn:1sid:zoobank.org:pub:18DB5F54-5CEB-498E-A6F1-E570E6A57833
}

\title{
Systematic revision of the parasitoid wasp genus Glyptapanteles Ashmead (Hymenoptera: Braconidae: Microgastrinae) for Australia results in a ten-fold increase in species
}

\author{
Erinn P. FAGAN-JEFFRIES ${ }^{\circledR 1, *}$, Alana R. McCLELLAND ${ }^{\circledR 2}$ Andrew J. BIRD ${ }^{\circledR 3}$, \\ Madalene M. GIANNOTTA ${ }^{\circledR 4}{ }^{4}$, Tessa M. BRADFORD ${ }^{\circledR 5}{ }^{5}$ \& Andrew D. AUSTIN ${ }^{\circledR} 6$ \\ 1,2,3,5,6 Australian Centre for Evolutionary Biology \& Biodiversity and School of Biological Sciences, \\ The University of Adelaide, Australia. \\ 1,5,6 South Australian Museum, Adelaide, Australia. \\ ${ }^{4}$ Australian National Insect Collection, CSIRO, Black Mountain, ACT, \\ Australia and Centre for Biodiversity Analysis, Research School of Biology, \\ Australian National University, Acton, ACT, Australia. \\ *Corresponding author: erinn.fagan-jeffries@adelaide.edu.au \\ ${ }^{2}$ Email: alana.mcclelland@adelaide.edu.au \\ ${ }^{3}$ Email: andrewbird@ozemail.com.au \\ ${ }^{4}$ Email: madalene.giannotta@gmail.com \\ ${ }^{5}$ Email: tessa.bradford@samuseum.sa.gov.au \\ ${ }^{6}$ Email: andrew.austin@adelaide.edu.au

\footnotetext{
${ }^{1}$ urn:lsid:zoobank.org:author:C724E269-029E-49E8-8D95-6F5A5DA6BAAF

${ }^{2}$ urn:lsid:zoobank.org:author:3FDC78D1-CDF3-472F-B4EE-63A43C1730AF

${ }^{3}$ urn:1sid:zoobank.org:author:DC97FEB2-1BB0-48CE-9178-0C5F98131CC0

${ }^{4}$ urn:Isid:zoobank.org:author:FF66BA72-4585-402F-AA42-61C9B7856048

${ }^{5}$ urn:lsid:zoobank.org:author:D018F430-ED59-47BA-BF6A-EF8C6675AC20

${ }^{6}$ urn:Isid:zoobank.org:author:DE71F924-750D-490D-84A7-F5960066F7CC
}

\begin{abstract}
Despite several decades of active research, there are still substantial gaps in the knowledge of parasitoid wasps in Australia, with many families and genera yet to be revised using modern approaches and only a fraction of the estimated fauna currently described. The genus Glyptapanteles Ashmead, 1904 is a member of the subfamily Microgastrinae (Hymenoptera: Braconidae) and all species in the subfamily are lepidopteran parasitoids. The genus previously contained only three species known from Australia: G. deliasa Austin \& Dangerfield, 1992, G. drioplanetus Fagan-Jeffries \& Austin, 2021 and G. mnesampela Austin, 2000. To undertake a revision of this morphologically-conserved group in Australia, we used a combination of molecular (cytochrome oxidase subunit one $(C O I)$ and wingless genes) and minimal morphological data to delimit and describe an additional 31 species: G. austini Fagan-Jeffries \& Bird sp. nov. and the following 30 species all authored by Fagan-Jeffries, Bird \& Austin: G. albigena sp. nov., G. andamookaensis sp. nov., G. arcanus sp. nov., G. aspersus sp. nov., G. austrinus sp. nov., G. baylessi sp. nov., G. bradfordae sp. nov., G. cooperi sp. nov., G. doreyi sp. nov., G. dowtoni sp. nov., G. eburneus sp. nov., G. erucadesolator sp. nov., G. ferrugineus sp. nov., G. foraminous sp. nov., G. goodwinnoakes sp. nov., G. guzikae sp. nov., G. harveyi sp. nov., G. kingae
\end{abstract}


sp. nov., G. kittelae sp. nov., G. kurandaensis sp. nov., G. lambkinae sp. nov., G. lessardi sp. nov., G. mouldsi sp. nov., G. niveus sp. nov., G. rixi sp. nov., G. rodriguezae sp. nov., G. ruhri sp. nov., G. sanniopolus sp. nov., G. vergrandiacus sp. nov. and G. wrightae sp. nov. We provide a key to species groups and to the species able to be identified on morphological characters alone. Additionally, we provide a brief discussion of the difficulties in describing small, morphologically conserved wasps and the challenges associated with revising the taxonomy of hyperdiverse taxa in the context of the planned mission of Taxonomy Australia to accelerate the documentation of Australia's biodiversity.

Keywords. Microgastrine, species delimitation, DNA barcoding, Australasia.

Fagan-Jeffries E.P., McClelland A.R., Bird A.J., Giannotta M.M., Bradford T.M. \& Austin A.D. 2022. Systematic revision of the parasitoid wasp genus Glyptapanteles Ashmead (Hymenoptera: Braconidae: Microgastrinae) for Australia results in a ten-fold increase in species. European Journal of Taxonomy 792: 1-116.

https://doi.org/10.5852/ejt.2022.792.1647

\section{Introduction}

Accelerating the documentation of Australia's biodiversity, in line with the decadal plan for Taxonomy in Australia and New Zealand (Taxonomy Decadal Plan Working Group 2018), requires the rapid description of new species of hyper-diverse groups of organisms. One such group are the parasitoid wasps, of which only a fraction are formally named (Yeates et al. 2003). Parasitoid wasps are important components of nearly all ecosystems, yet only fragmentary work has been completed on the systematics of most families in Australia. The Microgastrinae Foerster, 1962, a subfamily of the Braconidae Latreille, 1829, are thought to number in the tens of thousands of species (Rodriguez et al. 2013), with approximately 3000 species in 81 genera currently described worldwide (Fernández-Triana et al. 2020). In Australia, 143 species in 22 genera (Fagan-Jeffries \& Austin 2020; Fernández-Triana et al. 2020) are currently recognised, with 280 Barcode Index Numbers (BINs - operational taxonomic units which closely correspond to species) (Ratnasingham \& Hebert 2013) present on the Barcode of Life Database (BOLD) as of 24 July 2021. All microgastrines are parasitoids of lepidopteran larvae, are important in both regulating native caterpillar populations and as current and potential biological control agents of agricultural pests.

The genus Glyptapanteles Ashmead, 1904 has only three species described from Australia: G. deliasa Austin \& Dangerfield, 1992, G. drioplanetus Fagan-Jeffries \& Austin, 2021 and G. mnesampela Austin, 2000. Worldwide, there are 307 species described of an estimated 3000+, with the genus being found in all biogeographical regions (Fernández-Triana et al. 2020). The limits of the genus are controversial, with close morphological similarities to Cotesia Cameron, 1891, Distatrix Mason, 1981, Lathrapanteles Williams, 1985, Protapanteles Ashmead, 1898 and Sathon Mason, 1981 (Arias-Penna et al. 2019). In Australia, the genus, as currently recognised, is clearly paraphyletic with respect to Cotesia based on sequence data from cytochrome c oxidase subunit 1 (COI) and wingless genes (Fagan-Jeffries et al. 2018; Fagan-Jeffries \& Austin 2020). Despite the likelihood that the genus is not monophyletic, Glyptapanteles can normally be separated from Cotesia using morphology. Cotesia has the first metasomal tergite parallel-sided and the second metasomal tergite normally broad and rectangular, only ever slightly triangular, whereas Glyptapanteles often has a strongly narrowing first tergite and strongly triangular second tergite.

Of the other genera mentioned above, Distatrix has the median area on T2 less well-defined than in Glyptapanteles and has not been recorded from the Australasian region; Lathrapanteles is not a natural grouping and is not yet recorded from Australia and we see no reason to place more species here until a better classification of the subfamily is established; and Protapanteles is not yet recorded from Australia 
and has the first metasomal tergite parallel-sided except for a strongly rounded apex (Arias-Penna et al. 2019). Whilst Sathon in other parts of the world has the fore wing areolet absent (like in Glyptapanteles), in Australia all species currently placed in Sathon have a large closed areolet and are most likely unrelated to the northern hemisphere species (Austin \& Dangerfield 1992; Fagan-Jeffries et al. 2019). As such, Glyptapanteles in Australia is a relatively easily recognised group of species despite its paraphyletic nature and we feel it is untenable to split the genus into smaller units until a more thorough global phylogenetic study is conducted. Current ongoing projects utilising ultraconserved element bait sets and exon capture next-generation sequencing techniques will hopefully provide better resolution on the relationships and limits of microgastrine genera in the foreseeable future.

Using COI and wingless DNA barcoding data and key morphological characters, we here describe 31 new species of Glyptapanteles from Australia and provide a dichotomous key to species. All new species are authored by Fagan-Jeffries, Bird \& Austin other than Glyptapanteles austini, which is authored by Fagan-Jeffries \& Bird. Each new species description includes DNA barcode information, high-resolution images and a diagnosis against other Glyptapanteles described from Australia. Members of the genus from the broader Australasian region (Fiji, Samoa, New Zealand and Papua New Guinea) are listed, illustrated and diagnosed against the newly described Australian species where possible. We focus here on providing descriptions that are useful for end-user identification purposes and are as efficient as possible within the confines of what is generally accepted by the hymenopteran taxonomic community in terms of minimalist descriptions. As morphology is often not particularly useful for species delimitation in this genus, as it is not in many groups of Microgastrinae, we provide morphological diagnoses where possible, but also rely on COI and wingless sequences to delimit and diagnose species.

This paper includes important material from the 'Bush Blitz' surveys of regional Australia, a multiinstitutional partnership coordinated by the Federal Government's Australian Biological Resources Study (ABRS). The 'Bush Blitz' program brings together taxonomists, traditional landowners, property managers and park rangers to intensely survey the flora and fauna of reserves across the continent and describe the new species discovered during the process.

\section{Material and methods}

As the aim of the Taxonomy Australia decadal plan is to increase the number of species described in Australia in the most efficient and robust way possible, we use molecular information to inform the species prioritised for description in this study. From a large dataset of both published and unpublished $\mathrm{COI}$ and wingless sequences of Australian microgastrines, the Glyptapanteles sequences were extracted using a combination of BOLD identifications and the morphological examination of specimens. The previously unpublished DNA sequences used in this study were generated following the high-throughput methods outlined in Fagan-Jeffries et al. (2018) and are now uploaded to BOLD.

\section{Placing the Australian Glyptapanteles in the global context}

All of the available COI sequences of specimens identified as Glyptapanteles on BOLD as of 5 June $2021(\mathrm{n}=5289)$ were aligned using MAFFT ver. 7.308 (Katoh et al. 2002; Katoh \& Standley 2013). A Maximum Likelihood (ML) analysis was conducted on all of the unique sequences $(n=2558)$ using IQTREE ver. 1.6.12 (Nguyen et al. 2015) with models and partitions selected using the inbuilt ModelFinder (Kalyaanamoorthy et al. 2017) with the -TESTMERGE function, with a specimen of Microplitis demolitor Wilkinson, 1934 as the outgroup. Ultrafast bootstraps (1000 replicates) (Hoang et al. 2018) and SH-aLRT support (1000 replicates) were calculated for the tree. Full sequence details and the alignment are available as supplementary material (Supp. file 1, Supp. file 2). 


\section{Phylogenetics of the Australian Glyptapanteles}

BOLD was used to collate a dataset of all Glyptapanteles from Australia, Fiji and Papua New Guinea (as of the 9 June 2021), with sequences of Cotesia from Australia included for the contextual placement of this genus. A concatenated COI + wingless ML phylogeny of 321 specimens was constructed using IQTREE ver. 1.6.12 (Nguyen et al. 2015) with models and partitions selected using the inbuilt ModelFinder (Kalyaanamoorthy et al. 2017) with the-TESTMERGE function, with a specimen of Microplitis demolitor as the outgroup. Ultrafast bootstraps (1000 replicates) (Hoang et al. 2018) and SH-aLRT support (1000 replicates) were calculated for the tree. Full sequence details and the alignment are available as supplementary material (Supp. file 3, Supp. file 4).

\section{Species delimitation}

An integrative approach to species delimitation using a general lineage species concept (de Queiroz 1998) was employed, with species being delimited based on a combination of their COI pairwise difference, wingless haplotype and distinct morphological characters. All clades with greater than a COI $2 \%$ pairwise difference (calculated using the percent identity function on the alignment in Geneious ver. 9.1.8) from each other were examined morphologically and most were recognised as new species. Some provisional species were not described, often because they contained only male specimens. Some described species are represented by single specimens and/or contain only damaged or incomplete specimens, but we feel that this approach optimises the number of valid species recognised as part of Australia's named biodiversity and the available molecular data will allow additional specimens to be easily assigned to these species in the future.

We also treat the described species of Glyptapanteles from Australia and species from the broader Australasian region, diagnosing them against the new species where possible. Without molecular data for these species to guide the delimitations, diagnosing some of these species was extremely difficult. We feel that with the mission to describe Australia's biodiversity within 25 years and considering the fact that only a very small proportion of the microgastrine species have been described without molecular data, it is better to describe new species even when they cannot be conclusively separated from historically described species of the region using morphology. We only decide to do this where there is a very low probability that species are synonymous (based on knowledge of the general overlap of species distribution between countries or other information presented under each species), acknowledging that it is possible there may be some synonymy required in the future when improved technologies allow the non-destructive DNA extraction from old type material to be more cost-effective and guaranteed of success.

\section{Morphology}

Terms for general morphology and wing venation follow Fagan-Jeffries \& Austin (2020) and terms for sculpture follow Eady (1968). Nearly all images of specimens and morphological characters were generated using a Visionary Digital BK + imaging system with a Canon EOS 7D 18 megapixel camera, compiled in Zerene Stacker (Zerene Systems LLC), PMax software and edited for clarity in Adobe Photoshop 2021 (Adobe Systems Inc., San Jose, CA, USA).

Measurements of morphological characters were completed using an ocular micrometre on a Leica Mz16 microscope under a $10 \times$ or $100 \times$ magnification. Characters which we consider particularly important for diagnoses but are prone to variability within a species (colouration, shape of tergites, number of pits in the scutellar sulcus, ocelli measurements, flagellomere length/width ratios, ovipositor length and sculpture - including of the propodeum, anteromesoscutum and tergites) were coded for every available female specimen. Characters that are traditionally included in hymenopteran descriptions but that we consider very prone to inaccurate measurement and therefore not particularly useful for diagnoses between species in the context of the Australian fauna, we measured only on the holotypes (wing vein 
ratios, tergite length/width ratios) and note that these characters, unless extremely divergent, should not be used as diagnostic characters in the future for these species.

\section{Abbreviations}

The following abbreviations are used throughout the text:

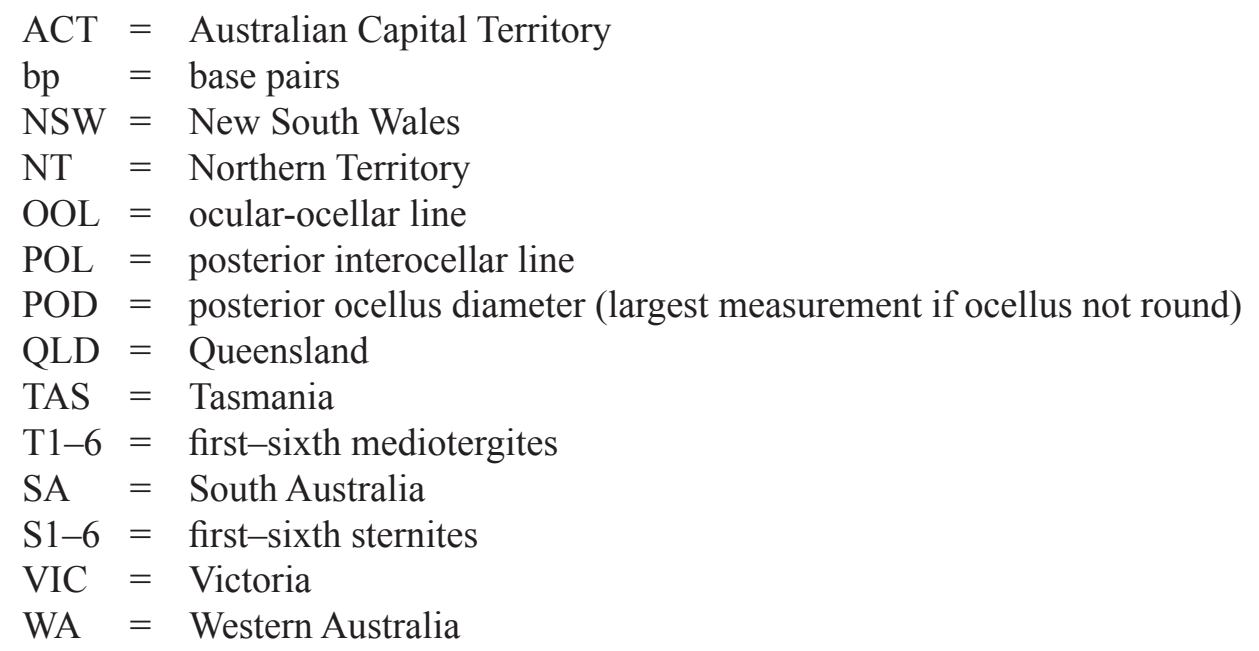

\section{Institutional abbreviations}

$\begin{array}{ll}\text { AM } & \text { Australian Museum, Sydney, Australia } \\ \text { ANIC } & =\text { Australian National Insect Collection, Canberra, Australia } \\ \text { BPBM } & \text { Bernice P. Bishop Museum, Honolulu, Hawaii, USA } \\ \text { NHMUK } & =\text { Natural History Museum, London, UK } \\ \text { QM } & \text { Queensland Museum, Brisbane, Australia } \\ \text { SAMA } & =\text { South Australian Museum, Adelaide, Australia } \\ \text { TMAG } & \text { Tasmanian Museum and Art Gallery, Hobart, Australia } \\ \text { WAM } & =\text { Western Australian Museum, Perth, Australia }\end{array}$

\section{Results}

\section{Molecular results}

A COI phylogeny of all sequences identified as Glyptapanteles on BOLD suggests that the Australian fauna are not a monophyletic group (Fig. 1). There are several clades that appear to be endemic to Australia or to Australia and Papua New Guinea, whilst there are also several species that are more closely related to specimens from Asia or South America than they are to other Australian lineages. Whilst COI alone does not necessarily provide reliable measures of relationships at these deeper levels, this preliminary phylogeny also suggests that the New Zealand fauna is a monophyletic group and not closely related to any sequenced Australian species (Fig. 1). The analysis also confirms that none of the Australian lineages described in this study are identical to previously sequenced specimens found in other regions, decreasing the possibility that the species described in this study overlap with taxa described from elsewhere. There is one Australian sequence sourced from BOLD (ASQAS186-11) that has the collection locality listed only as 'Australia', that is identical to sequences from Sweden, but we did not have access to this specimen and did not describe this species.

A COI + wingless ML analysis of the Glyptapanteles available on BOLD from Australia, Papua New Guinea and Fiji indicates there are more than 25 lineages yet to be described (Fig. 2) and highlights the paraphyletic nature of Glyptapanteles (as defined in this study for the Australian fauna) in relation to Cotesia. 


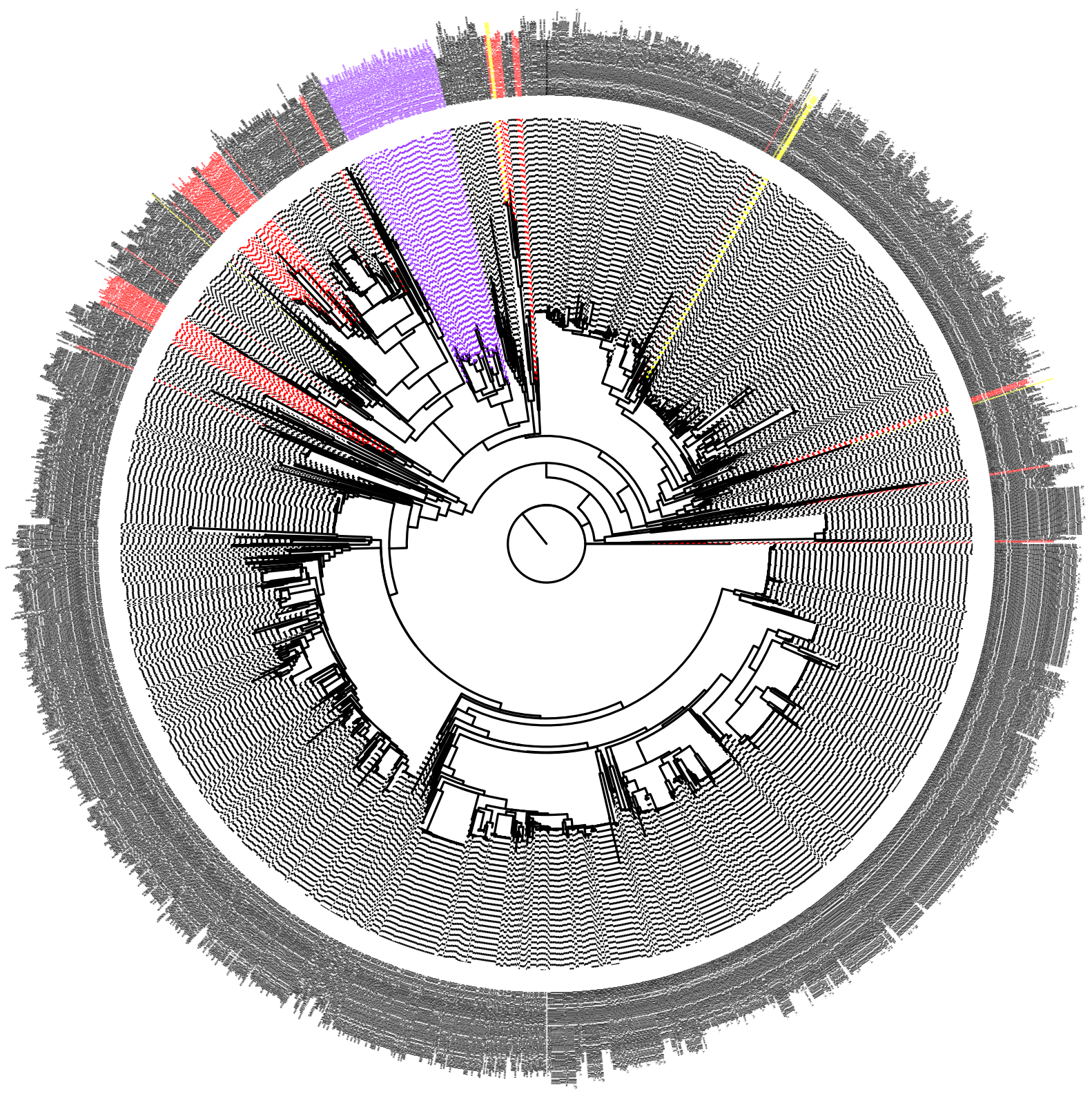

0.2

Fig. 1. COI ML phylogeny of all sequences identified as Glyptapanteles Ashmead, 1904 on BOLD as of 5 June 2021. Australian taxa are highlighted in red, New Zealand taxa in purple, Papua New Guinean taxa in yellow and taxa from Fiji in blue (only one lineage, difficult to discern in circular tree - see expanded phylogeny in Supp. file 5). Lineages in black are from elsewhere in the world. 


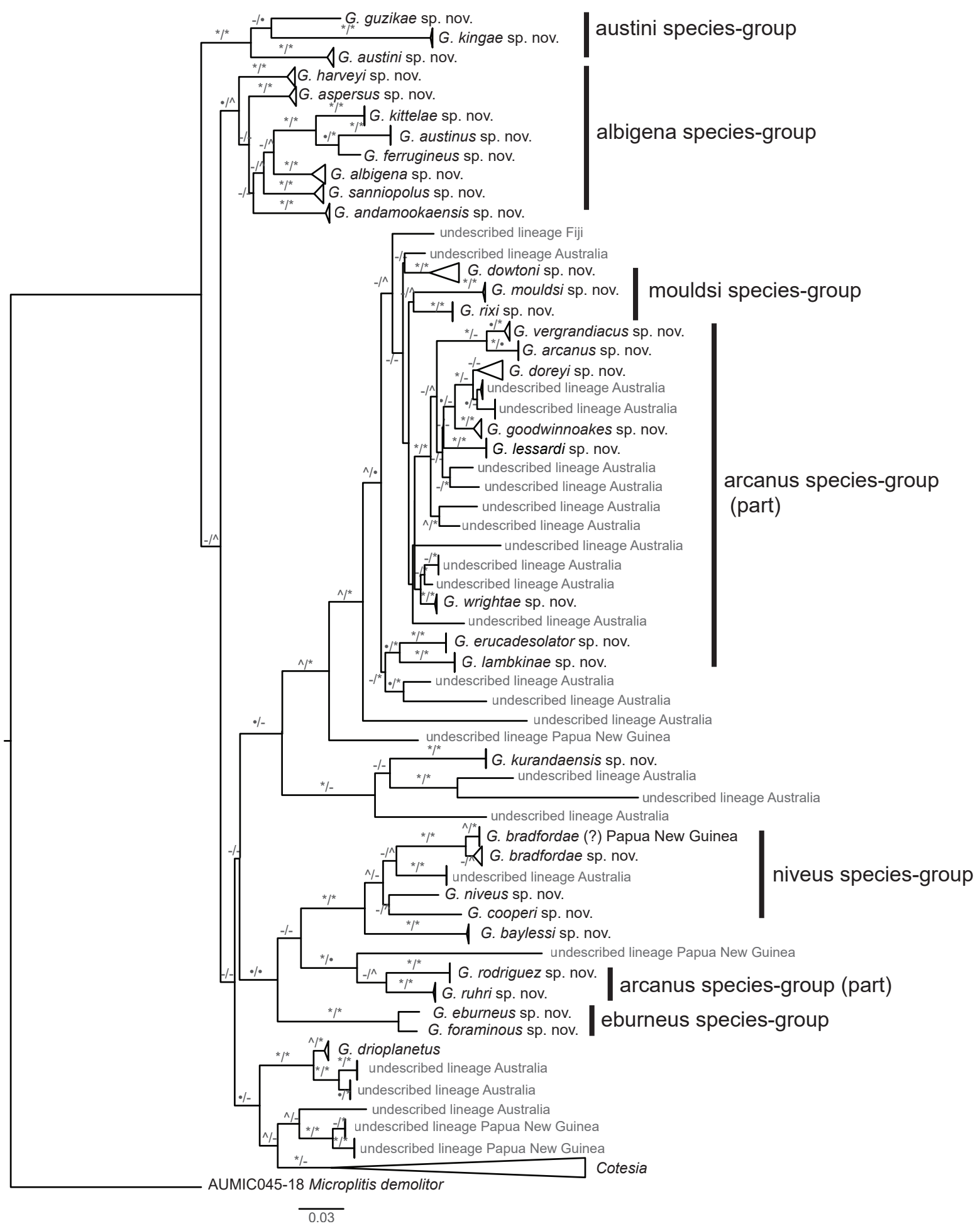

Fig. 2. Maximum likelihood phylogeny constructed using IQ-TREE ver. 1.6.12 of a concatenated COI and wingless alignment including Glyptapanteles Ashmead, 1904 from Australia, Papua New Guinea and Fiji, with specimens of Cotesia Cameron, 1891 from Australia included for contextual placement of the genus. Branch support values are given as SH-aLRT support (\%) / ultrafast bootstrap support (\%), with symbols representing value ranges as follows: $*=96-100 ; \bullet=91-95 ; \wedge=85-90 ;-=<85$. 

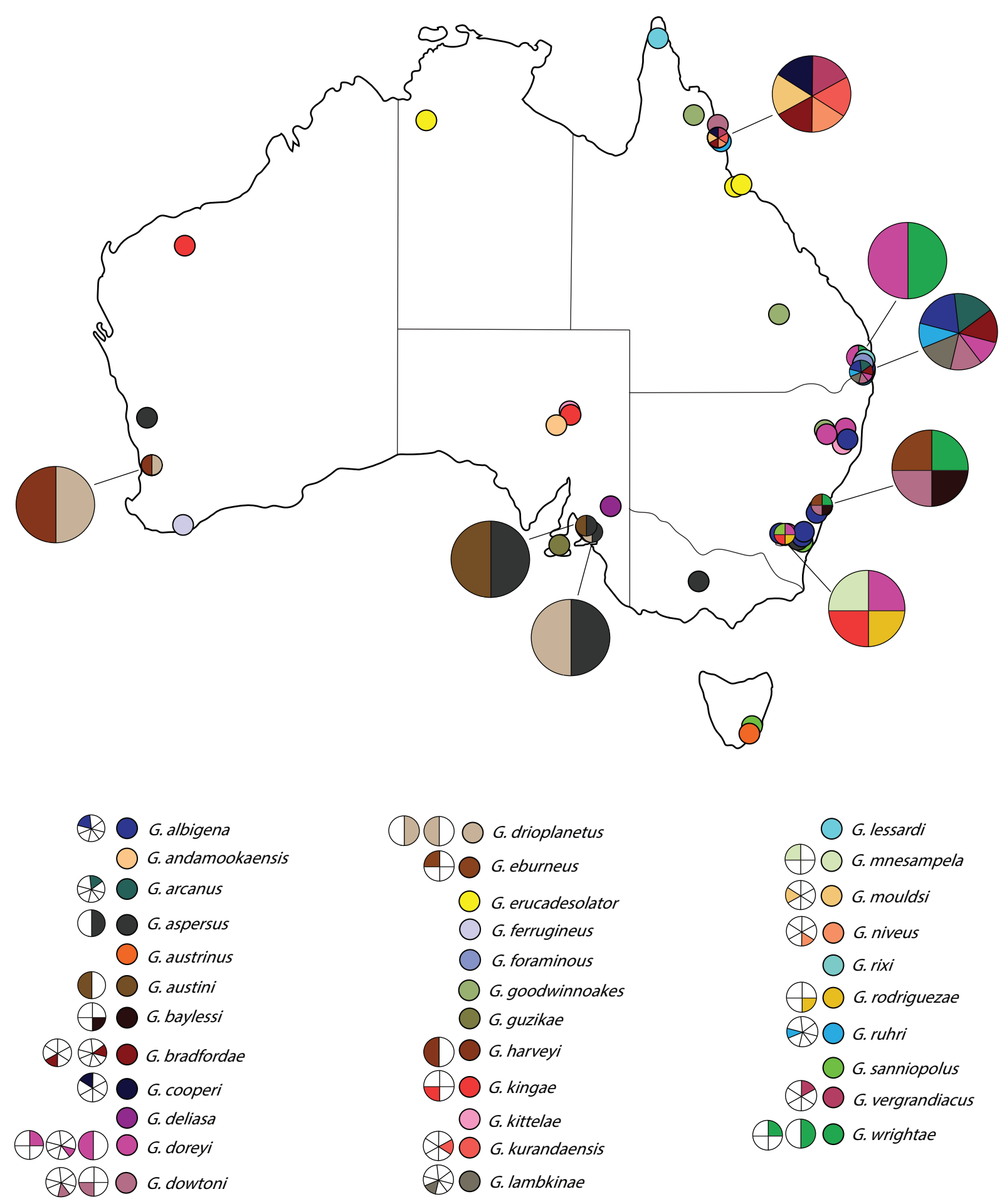

Fig. 3. Known distribution of the described species of Glyptapanteles Ashmead, 1904 from Australia, represented by coloured circles or part circles, with each species represented by a different colour (see key to colours below map). 


\section{Distribution of the described species of Glyptapanteles from Australia}

Sampling sites for this study were restricted to where there had been previous collections made into ethanol, allowing specimens to be DNA sequenced. The current known distribution of species of Glyptapanteles in Australia is therefore highly biased towards the recent field trips of researchers who deposit bulk samples into accessible state collections, or the field sites utilised by the study team and collaborators. The diversity of species at particular sites is also strongly biased towards collecting effort, with sites where long-term Malaise trapping occurred (e.g., Kuranda and Lamington National Park, both in Queensland; Fig. 3 represented by coloured wheels with at least six species) having a large number of species present. Sixteen of the 34 species of Glyptapanteles found in Australia are currently known from only one locality, which is most likely a reflection of limited sampling.

\section{Taxonomic treatment of species}

Class Insecta Linnaeus, 1758

Order Hymenoptera Linnaeus, 1758

Family Braconidae Latreille, 1829

Subfamily Microgastrinae Foerster, 1962

Genus Glyptapanteles Ashmead, 1904

Glyptapanteles Ashmead, 1904: 147.

Glyptapanteles - Mason 1981: 105. — Austin \& Dangerfield 1992: 32. — Arias-Penna et al. 2019a: 561. For complete list of earlier synonyms and bibliography, see Shenefelt (1972) and FernándezTriana et al. (2020).

\section{Type species}

By monotypy, Apanteles ashmeadi Wilkinson, 1928: 84, a replacement name for Glyptapanteles manilae Ashmead, 1904.

\section{Diagnosis}

The diagnosis by Fernández-Triana et al. (2020) works well for the Australian fauna and is as follows: "...fore wing without an areolet; propodeum that is either completely smooth (often) to more or less rugose (more rarely), with a median longitudinal carina that is entirely absent (often), partially defined posteriorly (often) to complete and strong (rarely), or no median carina but instead a series of very short carinae radiating from the nucha (rarely); T1 narrows towards the posterior margin, usually strongly (almost always), or more parallel-sided, or rounded at apex, as in some species of Protapanteles (rarely); T2 is almost always subtriangular or trapezoidal (rarely shaped differently); ovipositor and ovipositor sheaths are relatively short (usually) to moderately long (rarely); setae at apex of ovipositor sheaths relatively long (as long or longer than setae on hypopygium)" (Fernández-Triana et al. 2020: 36).

\section{Key to the described species groups and to the morphologically distinct species of Glyptapanteles from Australia}

This key is based on adult females. Due to there likely being many other undescribed species of Glyptapanteles in Australia, it should be treated with caution as undescribed species could possibly key to described species within this key. We feel that a morphological key is not particularly useful for this group, other than for separating the species groups which are quite distinct (e.g., the G. albigena species group, that has the gena with a pale spot), but we present one here for completeness. Ideally, morphological identifications should be supported with COI and wingless DNA barcodes. Species that come out together at a couplet are not reliably distinguished using morphology (e.g., couplet 7). 


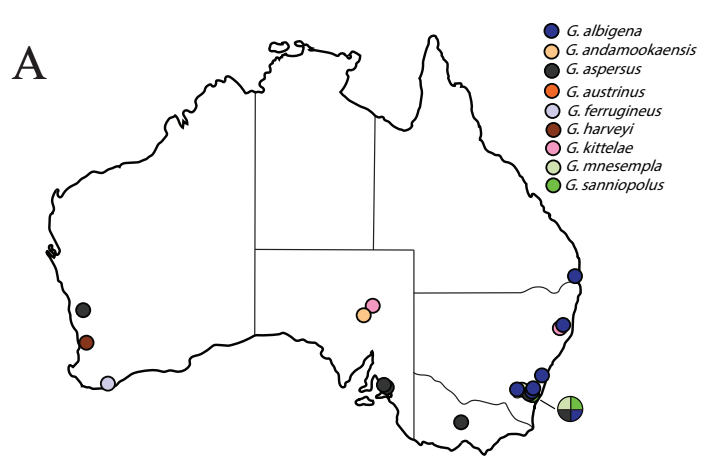

雨

C

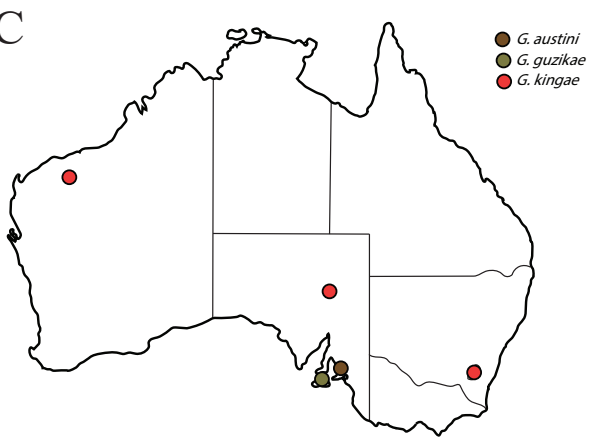

$\nabla$

$\mathrm{E}$

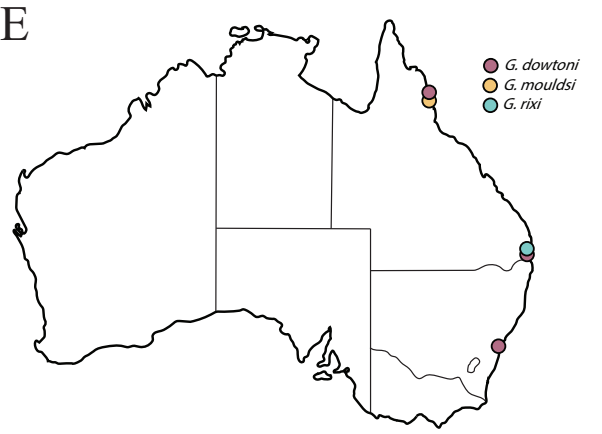

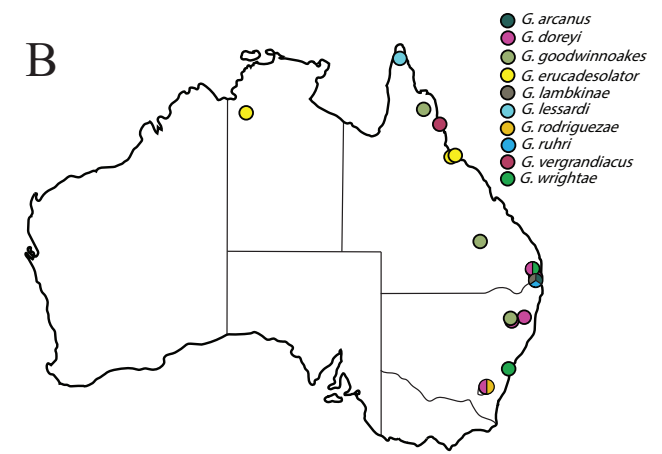

$\nabla$

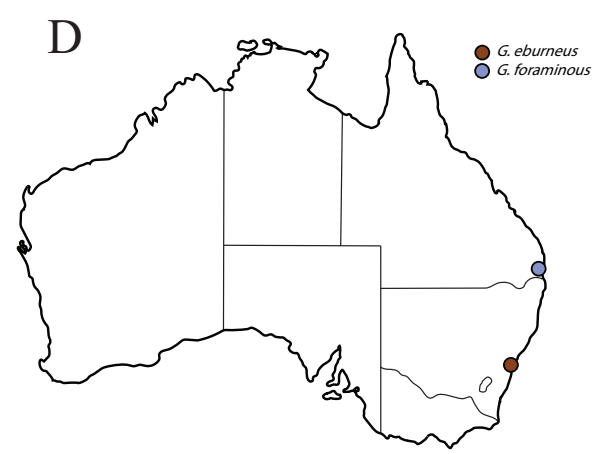

V

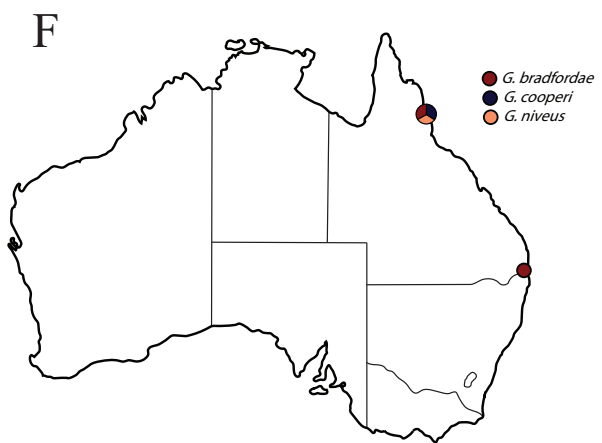

$\nabla$

G

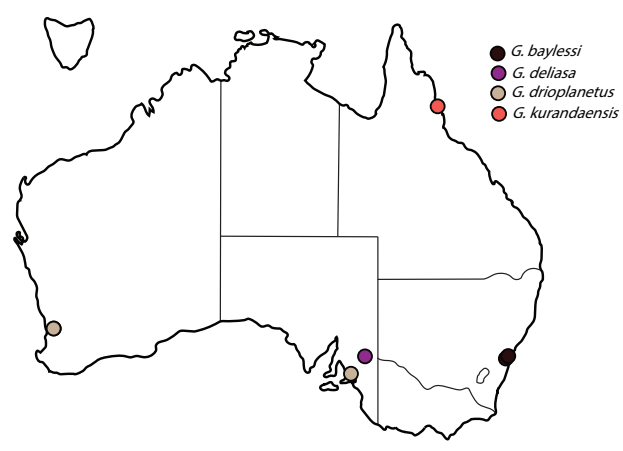

$\checkmark$

Fig. 4. Distribution of species groups of Glyptapanteles Ashmead, 1904 in Australia. A. G. albigena species group. B. G. arcanus species group. C. G. austini species group. D. G. eburneus species group. E. G. mouldsi species group. F. G. niveus species group. G. Unplaced species of Glyptapanteles in Australia. 
1. Gena with a pale spot (G. albigena species group) (Fig. 5A-D) .................................................. 2

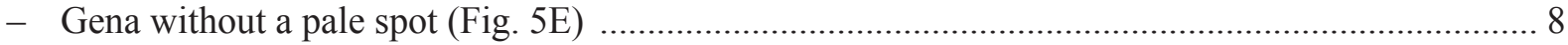

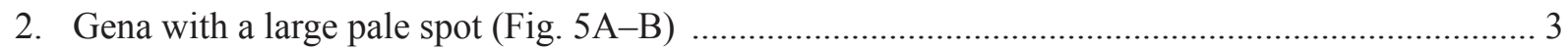

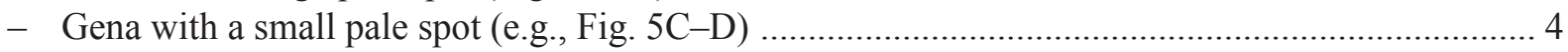

3. Gena with a pale spot measuring at least one third of face height (Fig. 5A)

Glyptapanteles albigena Fagan-Jeffries, Bird \& Austin sp. nov.

- Gena with a pale spot less than one third of face height (Fig. 5B)

Glyptapanteles sanniopolus Fagan-Jeffries, Bird \& Austin sp. nov.

4. T2 pale (e.g., Fig. 6A-E)

G. mnesampela Austin, 2000

- T2 dark (e.g., Fig. 6F)

5. Propodeum with median carina faintly indicated posteriorly and anteriorly (Fig. 7A); and gena with a barely visible pale spot (Fig. 5D) .......Glyptapanteles harveyi Fagan-Jeffries, Bird \& Austin sp. nov.

- Propodeum with median carina completely absent (e.g., Fig. 7B); gena with a clearly visible pale spot (e.g., Fig. 5C)

6. Hind femur mostly dark (sometimes with lighter area proximally) (Fig. 8A)

Glyptapanteles andamookaensis Fagan-Jeffries, Bird \& Austin sp. nov.

- Hind femur mostly light brown or pale (e.g., Fig. 8B)

7. Mesosoma with strong red tinge (Fig. 9A)

Glyptapanteles ferrugineus Fagan-Jeffries, Bird \& Austin sp. nov.

- Mesosoma dark, no strong red tinge (e.g., Fig. 9B)

Glyptapanteles kittelae Fagan-Jeffries, Bird \& Austin sp. nov.,

Glyptapanteles austrinus Fagan-Jeffries, Bird \& Austin sp. nov. and

Glyptapanteles aspersus Fagan-Jeffries, Bird \& Austin sp. nov.

These species cannot be reliably identified using morphology alone; see under the relevant species for molecularinformation which can be used to differentiate them
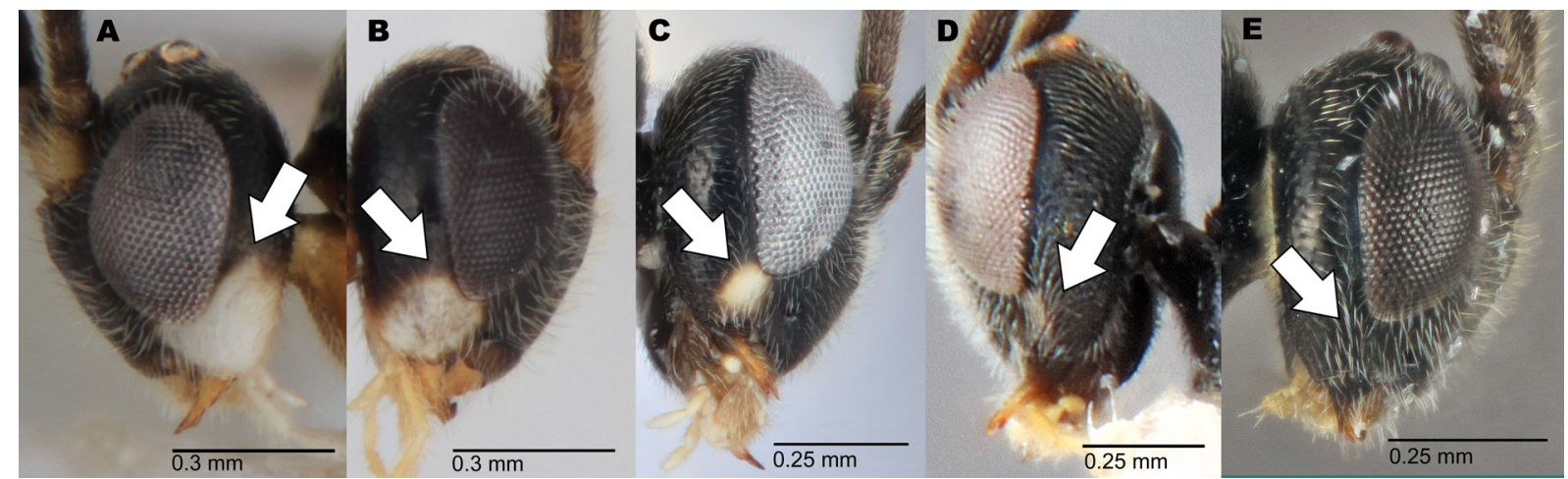

Fig. 5. A. Glyptapanteles albigena Fagan-Jeffries, Bird \& Austin sp. nov., holotype, $q$ (ANIC 32 130334), arrow indicating a large pale gena spot. B. G. sanniopolus Fagan-Jeffries, Bird \& Austin sp. nov., holotype, + (ANIC 32 130370), arrow indicating a large pale gena spot. C. G. kittelae FaganJeffries, Bird \& Austin sp. nov., holotype, $q$ (SAMA 32-46156) arrow indicating small (clearly visible) pale gena spot. D. G. harveyi Fagan-Jeffries, Bird \& Austin sp. nov., paratype, ㅇ (WAM E109889), arrow indicating small (faint, barely visible) pale gena spot. E. G. baylessi Fagan-Jeffries, Bird \& Austin sp. nov. paratype, $q$ (AM K.517936), gena without a pale spot. 

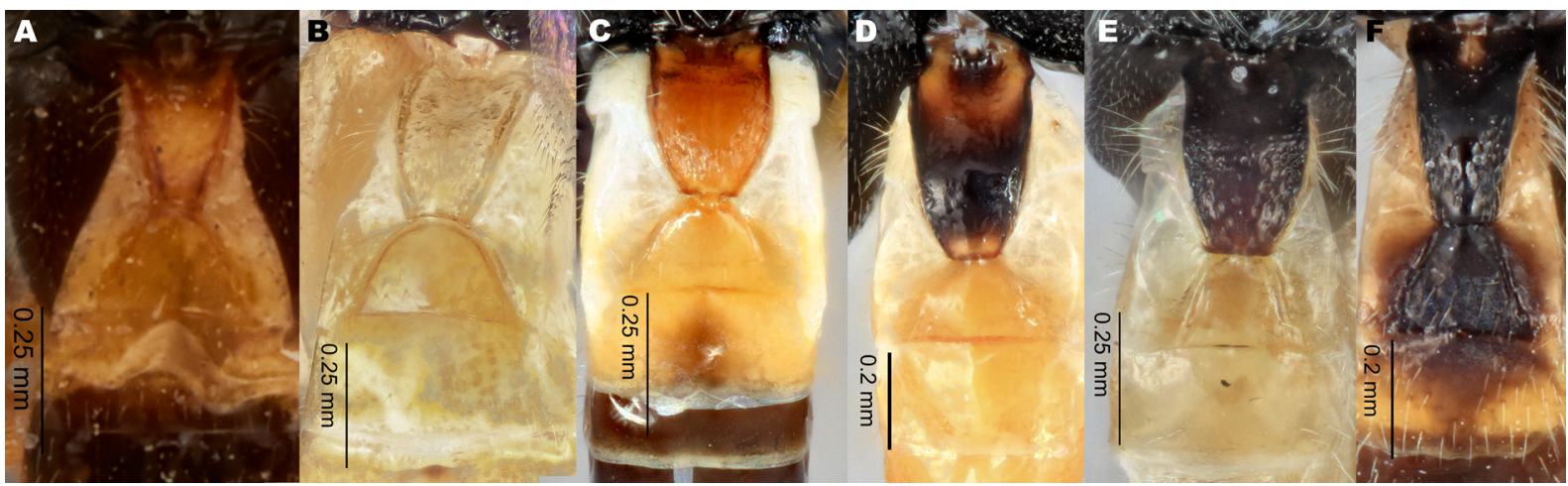

Fig. 6. A. Glyptapanteles mnesampela Austin, 2000, holotype, $q$ (ANIC 32-141445), T1 and T2 pale. B. G. eburneus Fagan-Jeffries, Bird \& Austin sp. nov., holotype, $ᄋ$ (AM K.517935), T1 and T2 pale. C. G. rixi Fagan-Jeffries, Bird \& Austin sp. nov., holotype, o+ (QM T250981), T2 pale, T1 darker than T2. D. G. mouldsi Fagan-Jeffries, Bird \& Austin sp. nov., paratype, ㅇ, (QM T250978), T1 dark, T2 pale. E. G. dowtoni Fagan-Jeffries, Bird \& Austin sp. nov., paratype, o (QM T250953), T1 dark, T2 pale. F. G. harveyi Fagan-Jeffries, Bird \& Austin sp. nov., paratype, + (WAM E109889), T1 dark, T2 dark.

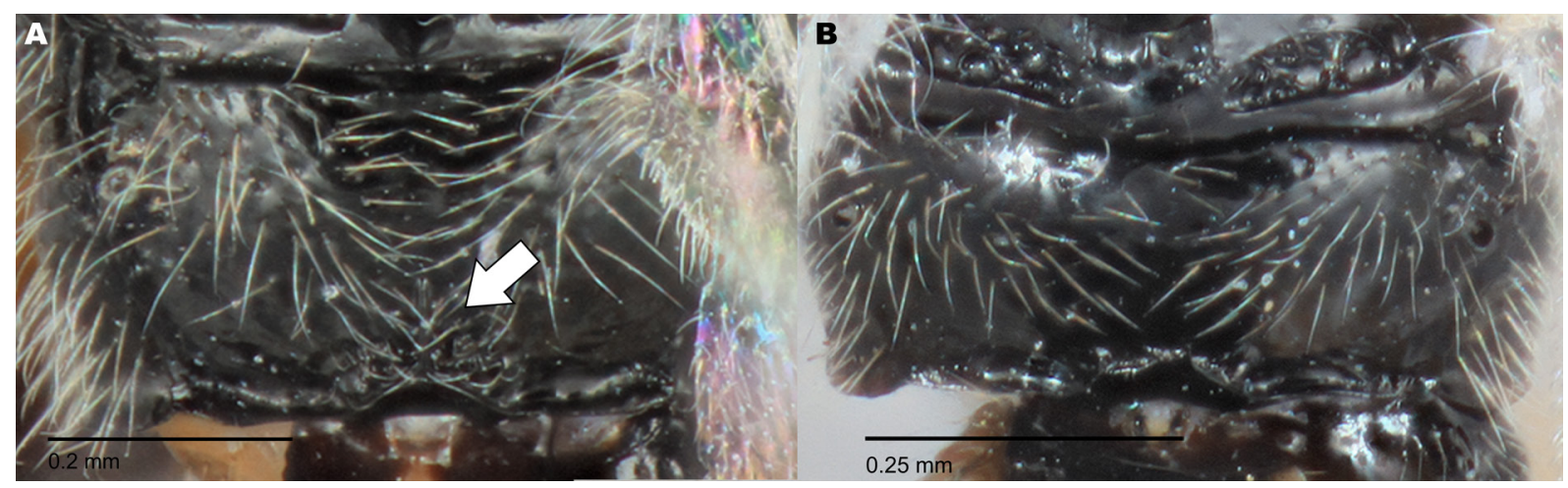

Fig. 7. A. Glyptapanteles harveyi Fagan-Jeffries, Bird \& Austin sp. nov., paratype, $q$ (WAM E109889), arrow indicating faint median carina at the posterior end of the propodeum. B. G. kittelae Fagan-Jeffries, Bird \& Austin sp. nov., holotype, $q$ (SAMA 32-46156), propodeum with median carina completely absent.

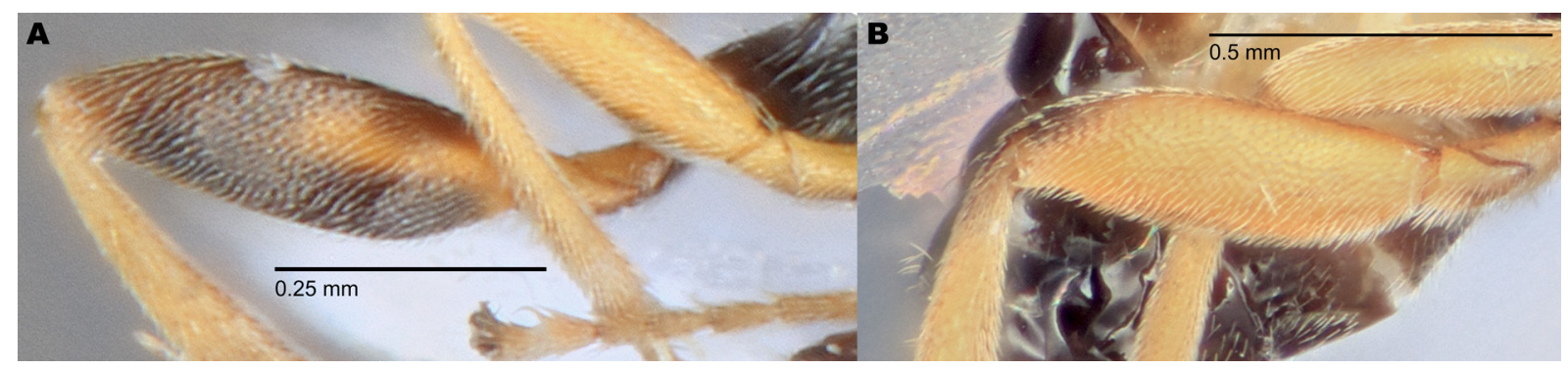

Fig. 8. A. Glyptapanteles andamookaensis Fagan-Jeffries, Bird \& Austin sp. nov., holotype, $q$ (SAMA 32-035451), hind femur mostly dark. B. G. kittelae Fagan-Jeffries, Bird \& Austin sp. nov., holotype, o (SAMA 32-46156), hind femur mostly light brown. 


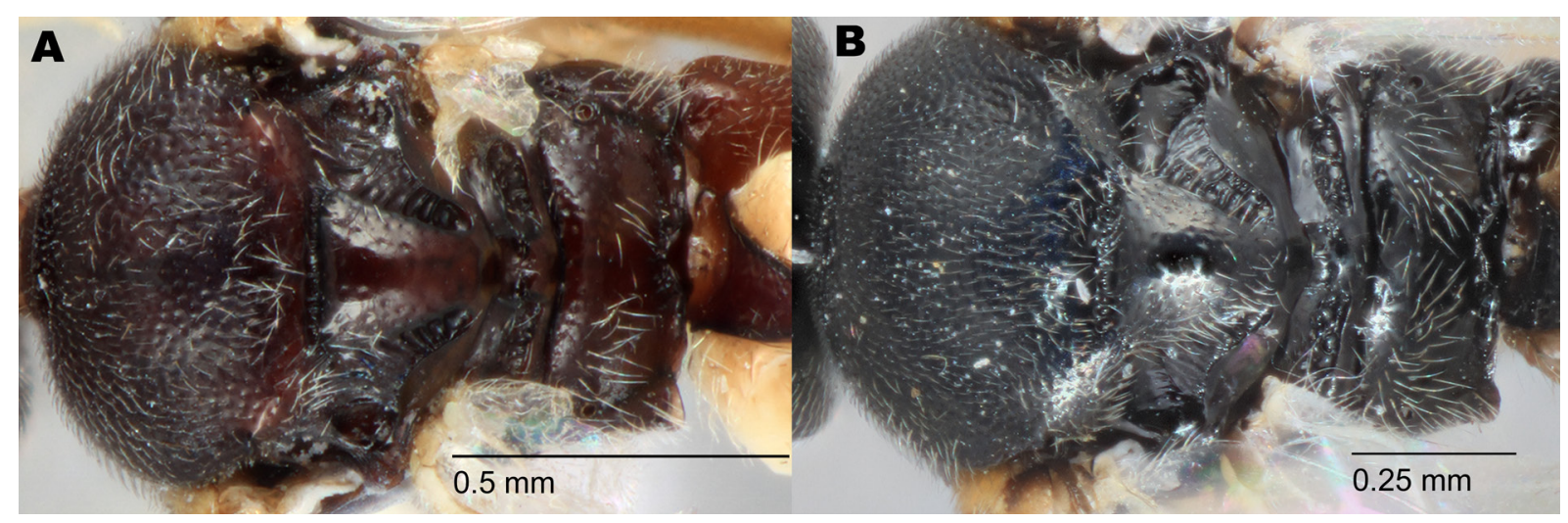

Fig. 9. A. Glyptapanteles ferrugineus Fagan-Jeffries, Bird \& Austin sp. nov., holotype, + (ANIC 32 130189), mesosoma with strong red tinge. B. G. kittelae Fagan-Jeffries, Bird \& Austin sp. nov., holotype, + (SAMA 32-46156), mesosoma dark, no strong red tinge.

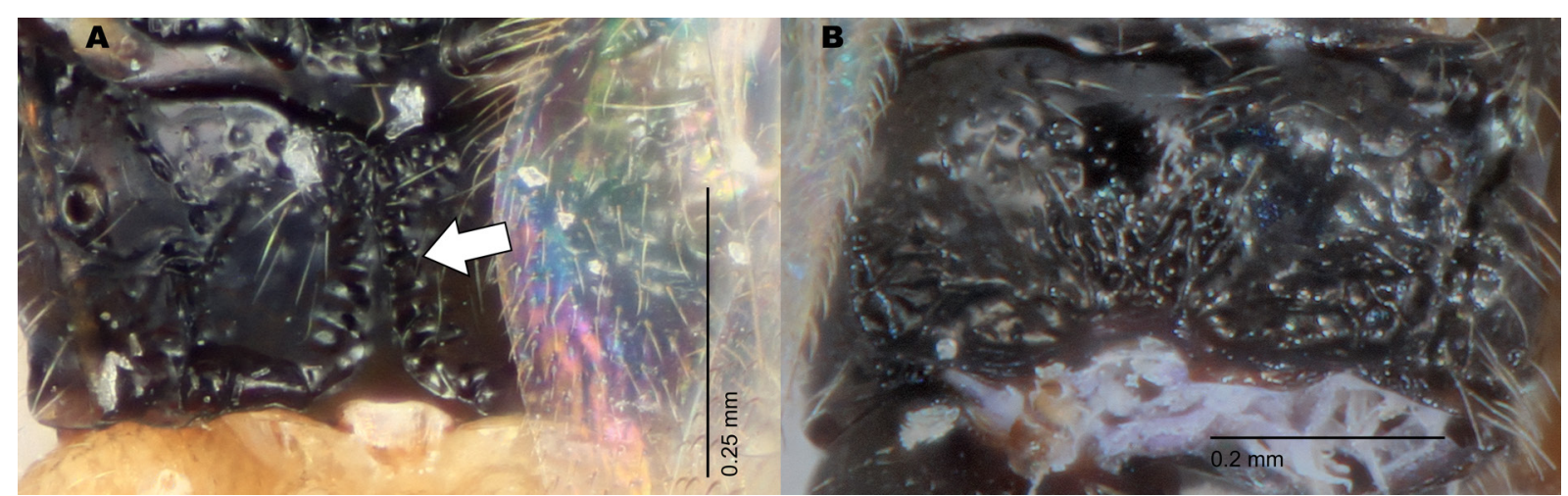

Fig. 10. A. Glyptapanteles eburneus Fagan-Jeffries, Bird \& Austin sp. nov., holotype, $q$ (AM K.517935), propodeum with median carina clear and complete (indicated by arrow). B. G. rixi Fagan-Jeffries, Bird \& Austin sp. nov., paratype, o (QM T250982), propodeum with median carina absent.

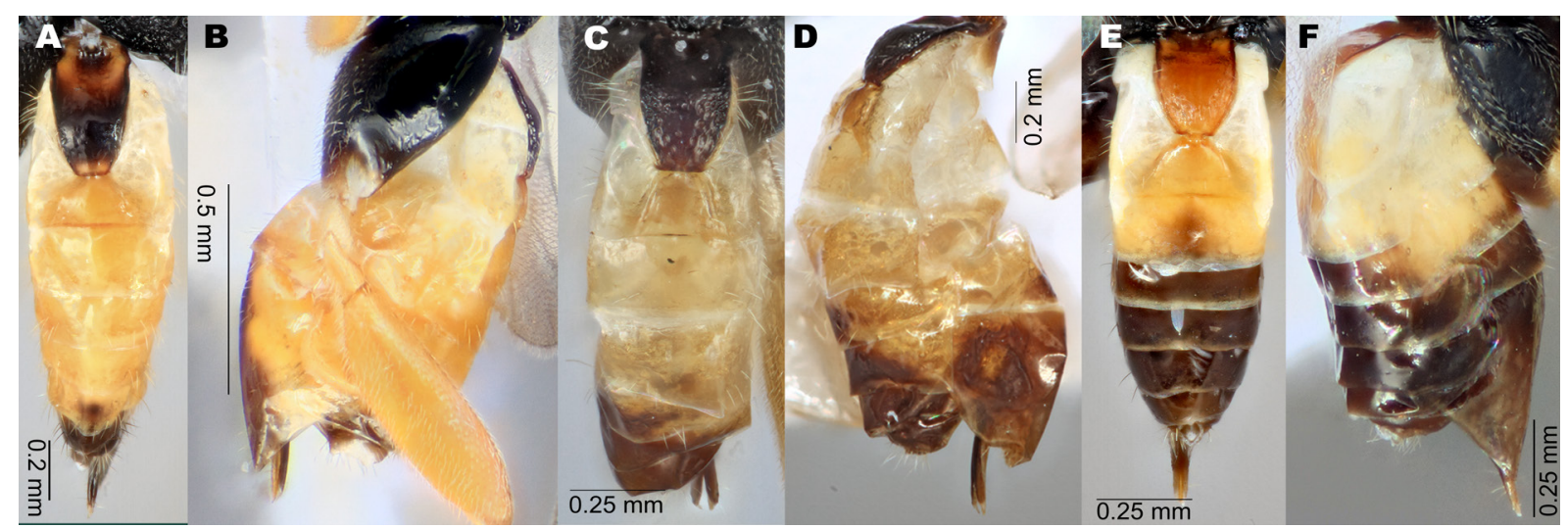

Fig. 11.A-B. Glyptapanteles mouldsi Fagan-Jeffries, Bird \& Austin sp. nov., paratype, $q$ (QM T250978). A. Dorsal metasoma. B. Lateral metasoma. C-D. G. dowtoni Fagan-Jeffries, Bird \& Austin sp. nov., paratype, + (QM T250953). C. Dorsal metasoma. D. Lateral metasoma. E-F. G. rixi Fagan-Jeffries, Bird \& Austin sp. nov., holotype, $q$ (QM T250981). E. Dorsal metasoma. F. Lateral metasoma. 


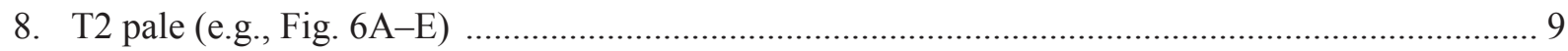

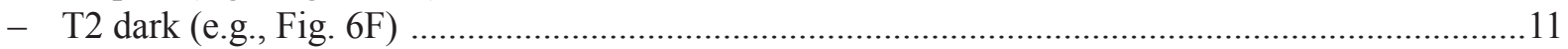

9. T1 same colour as T2, extremely pale (Fig. 6B); propodeum with median carina clear and complete; hind coxa pale (G. eburneus species group, part)

Glyptapanteles eburneus Fagan-Jeffries, Bird \& Austin sp. nov.

- T1 darker than T2, either dark or orange-brown (e.g., Fig. 6C-D); propodeum with median carina absent; hind coxa dark (G. mouldsi species group)

10. T1 dark, S3-5 mostly pale or yellow other than hypopygium (which is dark); T3-5 mostly pale or yellow (Fig. 11A-D) Glyptapanteles mouldsi Fagan-Jeffries, Bird \& Austin sp. nov. and

Glyptapanteles dowtoni Fagan-Jeffries, Bird \& Austin sp. nov.

These species cannot be reliably identified using morphology alone; see under the relevant species for molecular information which can be used to differentiate them

- T1 orange to light brown, S5-7 dark (including hypopygium); T4-6 completely dark (Fig. 11E-F)

Glyptapanteles rixi Fagan-Jeffries, Bird \& Austin sp. nov.

11. T1 pale; hind coxa pale (G. eburneus species group, part)

Glyptapanteles foraminous Fagan-Jeffries, Bird \& Austin sp. nov.

- T1 dark; hind coxa dark 12

12. Anteromesoscutum with very sparse deep punctures, always with some smooth areas greater than diameter of punctures; T1 never wedge-shaped, propodeum coarsely rugose; sometimes with slight orange markings on postero-lateral anteromesoscutum (G. niveus species group) (e.g., Fig. 12AB)

- Anteromesoscutum normally with punctures denser than in Fig. 12A-B, smooth areas generally smaller than diameter of punctures; T1 variable, including sometimes wedge-shaped, propodeum variable, coarsely rugose to smooth; sometimes with strong orange markings on postero-lateral anteromesoscutum (e.g., Fig. 12C-D)

13. Hind coxa with strong punctures covering most of area; antennal flagellomeres all dark

Glyptapanteles bradfordae Fagan-Jeffries, Bird \& Austin sp. nov.

- Hind coxa with only sparse punctures, particularly smooth anteriorly; antennal flagellomeres sometimes with white distal segments

Glyptapanteles cooperi Fagan-Jeffries, Bird \& Austin sp. nov. and

Glyptapanteles niveus Fagan-Jeffries, Bird \& Austin sp. nov.

These species cannot be reliably identified using morphology alone; see under the relevant species for molecular information which can be used to differentiate them

14. Postero-lateral anteromesoscutum with strong orange markings (Fig. 12C) [whole body strongly punctured, propodeum strongly rugose]

Glyptapanteles baylessi Fagan-Jeffries, Bird \& Austin sp. nov.

- Postero-lateral anteromesoscutum without strong orange markings (e.g., Fig. 12D) [sculpturing of propodeum variable, sometimes strongly rugose]

15. Ventral side of antennal scape (at least in distal half) darker than or the same colour as flagellomeres (e.g., Fig. 13A)

- Ventral side of antennal scape (at least in distal half) paler than flagellomeres (G. arcanus species group) (e.g., Fig. 13B) 
16. Fore wing veins $r$ and $2 R S$ narrow and long, each significantly longer than vein $2 \mathrm{~m}$ and smoothly curved (not meeting at sharp angle) (Fig. 14A)

Glyptapanteles kurandaensis Fagan-Jeffries, Bird \& Austin sp. nov.

- Fore wing veins $r$ and $2 \mathrm{RS}$ each only slightly longer than vein $2 \mathrm{~m}$ (Fig. 14B), sometimes meeting at sharp angle

17. Tegula dark

G. deliasa Austin \& Dangerfield, 1992

- Tegula pale

18. Maximum height of mesoscutellum lunules less than $0.64 \times$ maximum height of lateral face of mesoscutellum (Fig. 15A) [hind femur darkening posteriorly, propodeum smooth]

Glyptapanteles drioplanetus Fagan-Jeffries \& Austin, 2021

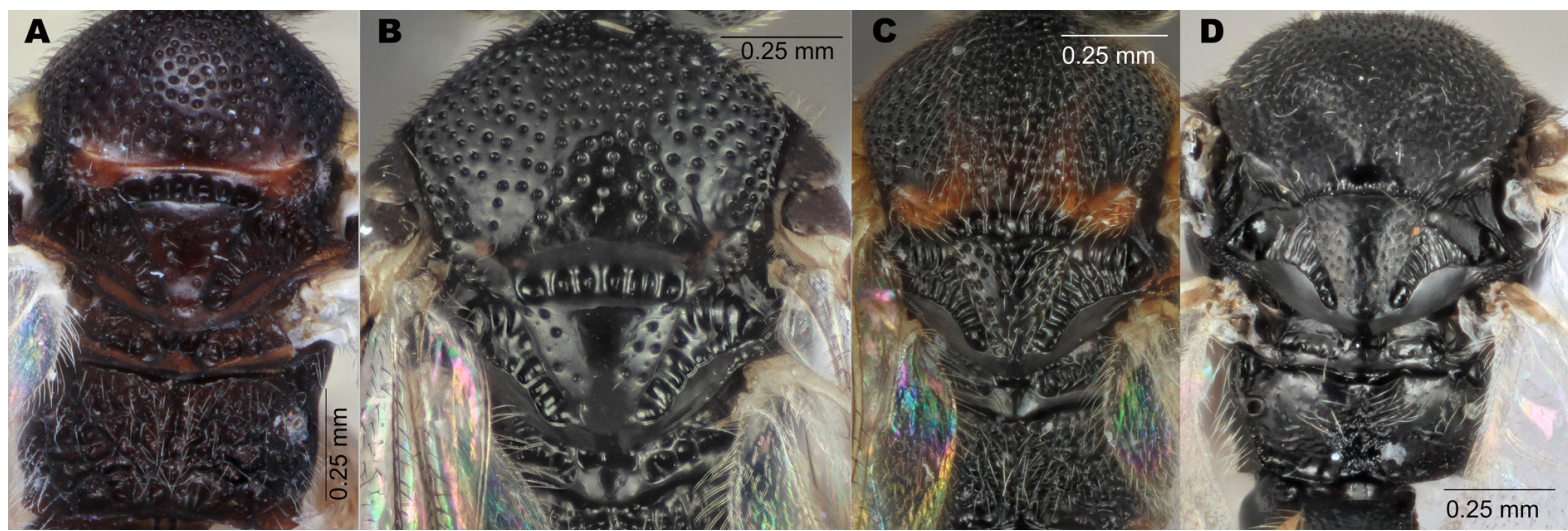

Fig. 12. A. Glyptapanteles bradfordae Fagan-Jeffries, Bird \& Austin sp. nov., paratype, $q$ (QM T250944), anteromesoscutum with very sparse deep punctures and slight orange markings. B. G. cooperi FaganJeffries, Bird \& Austin sp. nov., holotype, o (QM T250947), anteromesoscutum with very sparse deep punctures and no orange markings. C. G. baylessi Fagan-Jeffries, Bird \& Austin sp. nov., paratype, $q$ (AM K.517936), anteromesoscutum with dense punctures, strong orange markings on the postero-lateral corners. D. G. kurandaensis Fagan-Jeffries, Bird \& Austin sp. nov., paratype, + (QM T250971), with dense shallow punctures, but no strong orange markings on the anteromesoscutum.
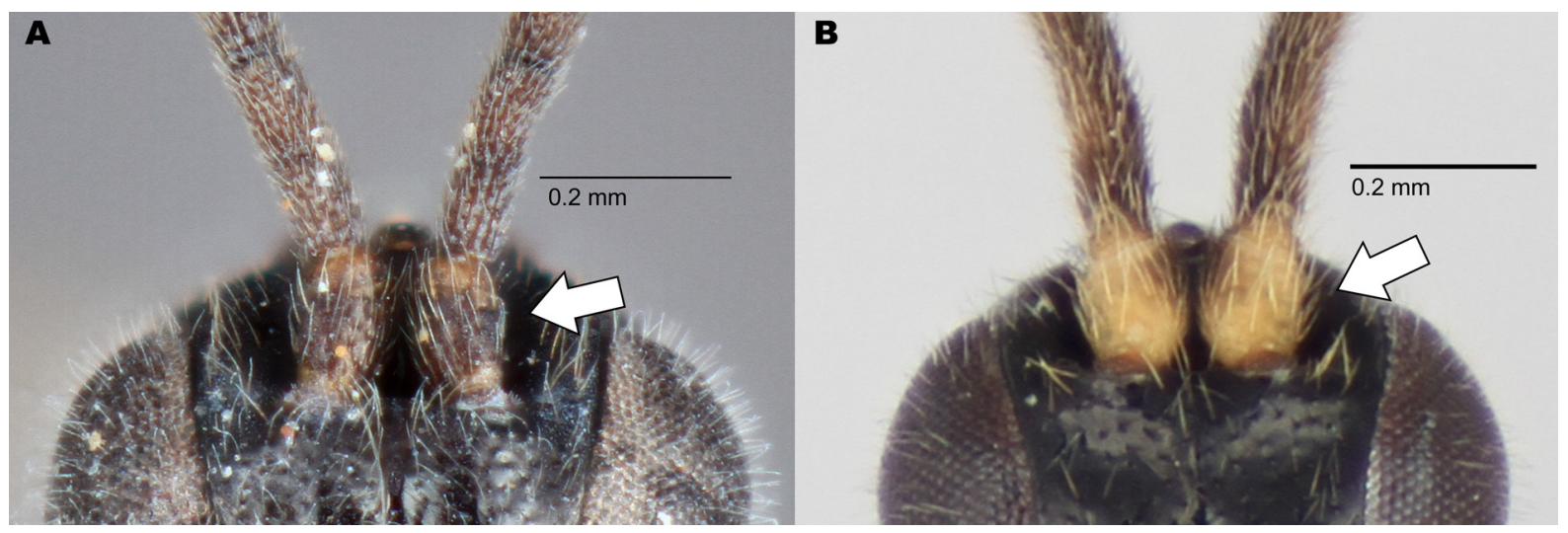

Fig. 13. A. Glyptapanteles deliasa Austin \& Dangerfield, 1992, paratype, $q$ (WINC), ventral half of antennal scape darker than flagellomeres. B. G. ruhri Fagan-Jeffries, Bird \& Austin sp. nov., holotype, $q$ (QM T250986), ventral half of antennal scape paler than flagellomeres. 
- Maximum height of mesoscutellum lunules approximately $0.75 \times$ maximum height of lateral face of mesoscutellum (e.g., Fig. 15B) [hind femur completely dark or mostly pale yellow with dark patch posteriorly, propodeum smooth or with some shallow indistinct sculpturing in centre] (G. austini species group)

19. Hind femur mostly pale with dark area posteriorly [propodeum smooth and shiny]

Glyptapanteles guzikae Fagan-Jeffries, Bird \& Austin sp. nov.

- Hind femur completely dark [propodeum smooth or with some sculpturing in centre]

Glyptapanteles kingae Fagan-Jeffries, Bird \& Austin sp. nov. and

Glyptapanteles austini Fagan-Jeffries \& Bird sp. nov.

These species cannot be reliably identified using morphology alone; see under the relevant species for molecular information which can be used to differentiate them

20. Propodeum with coarse rugose sculpturing over most of area

- Propodeum smooth, or with rugose sculpturing only in posterior half of centre, anterior half punctured or smooth

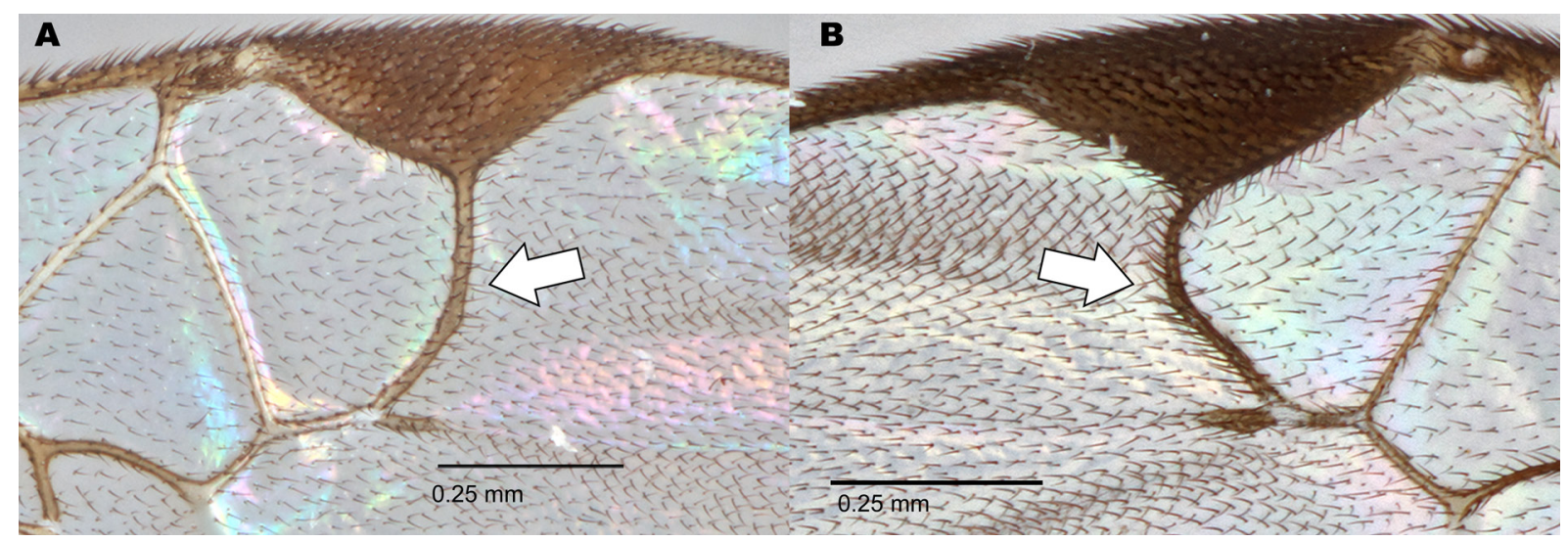

Fig. 14. A. Glyptapanteles kurandaensis Fagan-Jeffries, Bird \& Austin sp. nov., paratype, + (QM T250969), fore wing, arrow indicating vein r. B. G. kingae Fagan-Jeffries, Bird \& Austin sp. nov., holotype (SAMA 32-46154), arrow indicating vein $r$.
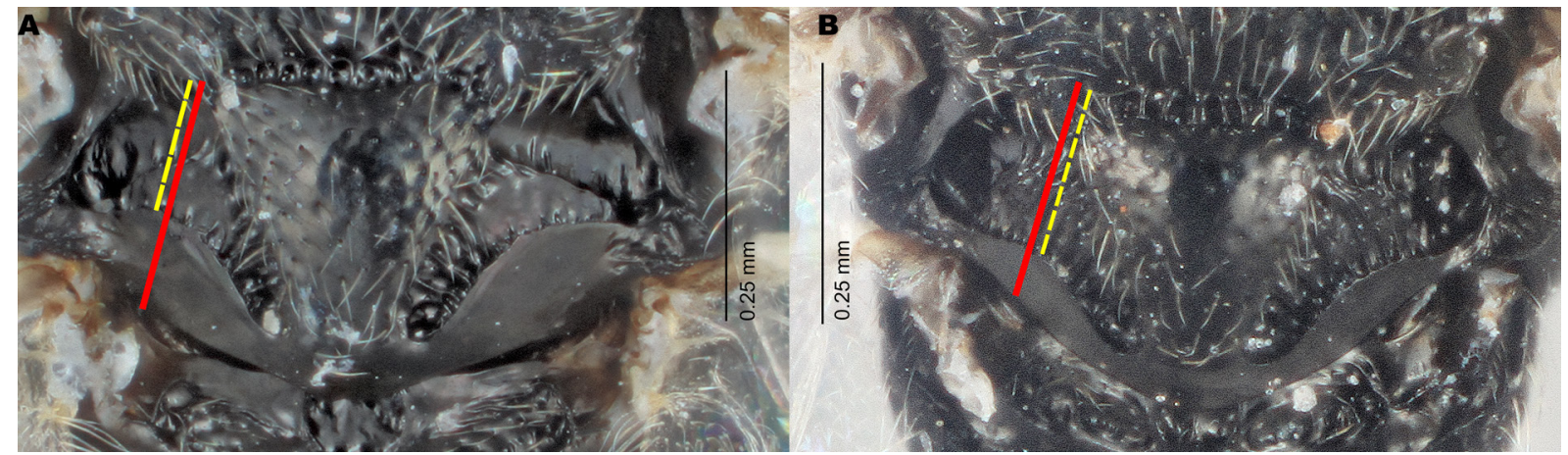

Fig. 15. A. Glyptapanteles drioplanetus Fagan-Jeffries \& Austin, 2021 (SAMA 32-45154). B. G. austini Fagan-Jeffries \& Bird sp. nov., paratype, o (SAMA 32-46151). Solid red line indicating lateral face of the mesoscutellum, yellow dashed line indicating mesoscutellum lunules. 
21. T1 smooth Glyptapanteles goodwinnoakes Fagan-Jeffries, Bird \& Austin sp. nov.

- T1 with strong or shallow rugose sculpturing

Glyptapanteles lambkinae Fagan-Jeffries, Bird \& Austin sp. nov., Glyptapanteles arcanus Fagan-Jeffries, Bird \& Austin sp. nov., Glyptapanteles vergrandiacus Fagan-Jeffries, Bird \& Austin sp. nov. and Glyptapanteles erucadesolator Fagan-Jeffries, Bird \& Austin sp. nov. These species cannot be reliably identified using morphology alone; see under the relevant species for molecular information which can be used to differentiate them

22. T1 with strong punctures in posterior half, punctures covering at least one third of posterior half of tergite

Glyptapanteles rodriguezae Fagan-Jeffries, Bird \& Austin sp. nov. and Glyptapanteles ruhri Fagan-Jeffries, Bird \& Austin sp. nov.

These species cannot be reliably identified using morphology alone; see under the relevant species for molecular information which can be used to differentiate them

- T1 smooth and shiny, if punctures present then only very few and very sparse or confined to lateral edges, covering much less than one third of area of posterior half of tergite

Glyptapanteles wrightae Fagan-Jeffries, Bird \& Austin sp. nov., Glyptapanteles doreyi Fagan-Jeffries, Bird \& Austin sp. nov. and

Glyptapanteles lessardi Fagan-Jeffries, Bird \& Austin sp. nov.

These species cannot be reliably identified using morphology alone; see under the relevant species for molecular information which can be used to differentiate them

\section{Species groups assignments and diagnoses}

Six species groups are designated based on a combination of molecular and morphological data. Diagnoses for these groups are given below and all of the treated species are then presented in alphabetical order.

\section{Glyptapanteles albigena species group}

The G. albigena species group is easily separated from the other species of Glyptapanteles in Australasia by the gena having a pale spot. This pale spot varies from barely visible (e.g., G. harveyi sp. nov.), to a large area covering at least a third of the gena height (e.g., G. albigena sp. nov.). This species group contains nine species: G. albigena Fagan-Jeffries, Bird \& Austin sp. nov., G. andamookaensis Fagan-Jeffries, Bird \& Austin sp. nov., G. aspersus Fagan-Jeffries, Bird \& Austin sp. nov., G. austrinus Fagan-Jeffries, Bird \& Austin sp. nov. sp. nov., G. ferrugineus Fagan-Jeffries, Bird \& Austin sp. nov., G. harveyi Fagan-Jeffries, Bird \& Austin sp. nov., G. kittelae Fagan-Jeffries, Bird \& Austin sp. nov., G. mnesampela Austin, 2000 and G. sanniopolus Fagan-Jeffries, Bird \& Austin sp. nov. The species group, as currently known, has a broad distribution across the southern half of Australia (Fig. 4A).

\section{Glyptapanteles arcanus species group}

The large G. arcanus species group forms two separate clades in the current phylogeny (Fig. 2) and is therefore clearly not a monophyletic group. There is one small clade of two species (G. ruhri FaganJeffries, Bird \& Austin sp. nov. and G. rodriguezae Fagan-Jeffries, Bird \& Austin sp. nov. plus an undescribed lineage, with a sequence in BOLD) which is sister to the G. niveus species group clade, whilst the remainder of species are paraphyletic with respect to the G. mouldsi species group and include G. arcanus Fagan-Jeffries, Bird \& Austin sp. nov., G. doreyi Fagan-Jeffries, Bird \& Austin sp. nov., G. erucadesolator Fagan-Jeffries, Bird \& Austin sp. nov., G. goodwinnoakes Fagan-Jeffries, Bird \& Austin sp. nov., G. lambkinae Fagan-Jeffries, Bird \& Austin sp. nov., G. lessardi Fagan-Jeffries, Bird \& Austin sp. nov., G. vergrandiacus Fagan-Jeffries, Bird \& Austin sp. nov. and G. wrightae Fagan-Jeffries, Bird \& Austin sp. nov. The species group, as currently recognised, is distributed throughout the eastern 
coast of Australia, from the northern regions of QLD through to central NSW and the ACT. One species, G. erucadesolator sp. nov., is also known from northern NT (Fig. 4B).

Species in the G. arcanus species group can be separated from the other described species of Glyptapanteles in Australia by the combination of the following characters: gena without a pale spot, $\mathrm{T} 1$ and T2 dark, anteromesoscutum punctures not extremely sparse and deep (i.e., not as in the niveus species group), no orange markings on anteromesoscutum postero-laterally, propodeum either strongly sculptured (rugose) or with punctures in the anterior half and shallow rugose sculpturing in the posterior half, ventral side of antennal scape (at least in distal half) paler than flagellomeres, tegula pale.

\section{Glyptapanteles austini species group}

The G. austini species group contains three species: G. austini Fagan-Jeffries \& Bird sp. nov., G. guzikae Fagan-Jeffries, Bird \& Austin sp. nov. and G. kingae sp. nov., which are a monophyletic, molecularly distinct lineage (Fig. 2). Two species in this group (G. guzikae sp. nov. and G. austini sp. nov.) are known from single localities in southern SA, whilst $G$. kingae sp. nov. has a broad distribution and is found in the northern half of WA, central SA and the ACT (Fig. 4C). The group can be separated from the other species of Glyptapanteles described from Australia by the following combination of characters: gena without a pale spot, T1-T2 dark, anteromesoscutum all dark and with punctures without smooth areas greater than the diameter of punctures (i.e., anteromesoscutum not as in the G. niveus species group (i.e., not as in Fig. 12B)), propodeum not coarsely rugose, T1 only parallel for at most $2 / 3$ of length before narrowing posteriorly, mesoscutellar disk without dense, strong punctures, scape darker or the same colour as flagellomeres, tegula pale, labrum dark.

\section{Glyptapanteles eburneus species group}

The G. eburneus species group currently contains only two closely related species, G. eburneus FaganJeffries, Bird \& Austin sp. nov. and G. foraminous Fagan-Jeffries, Bird \& Austin sp. nov. which are differentiated from all other species of Glyptapanteles in Australia by having the gena without a pale spot, $\mathrm{T} 1$ completely pale, hind coxa pale, deep punctures on the anteromesoscutum and a clear median carina on the propodeum. This species group is known from two single localities on the east coast of Australia, in QLD and NSW (Fig. 4D).

\section{Glyptapanteles mouldsi species group}

The G. mouldsi species group can be distinguished from all of the other Glyptapanteles described from Australia by having the gena without a pale spot, T2 pale in colouration, with T1 darker than T2 and no median carina on the propodeum. It contains three newly described species: G. dowtoni Fagan-Jeffries, Bird \& Austin sp. nov., G. mouldsi Fagan-Jeffries, Bird \& Austin sp. nov. and G. rixi Fagan-Jeffries, Bird \& Austin sp. nov. The group is paraphyletic in the current phylogeny (Fig. 2). The G. mouldsi species group is currently only known from the east coast of Australia, from northern QLD to central NSW (Fig. 4E).

\section{Glyptapanteles niveus species group}

The G. niveus species group can be separated from the other described Glyptapanteles from Australia by having the gena without a pale spot, $\mathrm{T} 1$ and $\mathrm{T} 2$ dark, propodeum coarsely rugose and the anteromesoscutum with very sparse deep punctures, always with some smooth areas larger than the diameter of punctures, face strongly punctured and T1 not wedge-shaped. All the described species also have a vividly white fore coxa, mid coxa, hind trochanter and anterior metasomal sternites. The species group currently contains three described species: G. bradfordae Fagan-Jeffries, Bird \& Austin sp. nov., G. cooperi Fagan-Jeffries, Bird \& Austin sp. nov. and G. niveus Fagan-Jeffries, Bird \& Austin sp. nov. 
A

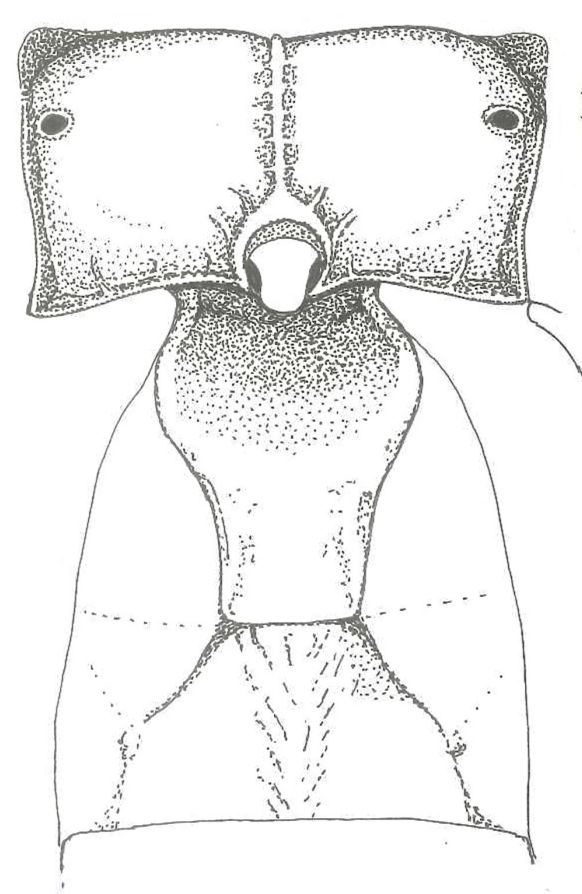

C

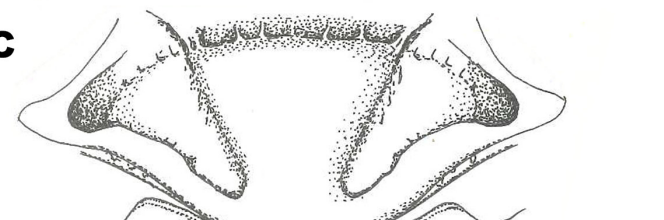

B

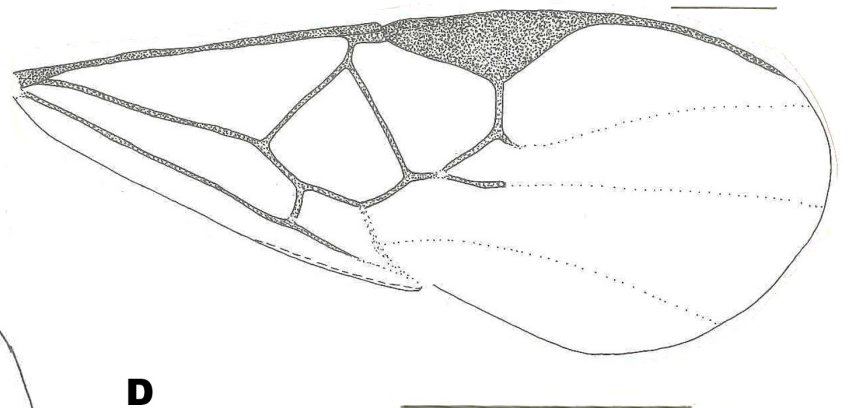

D
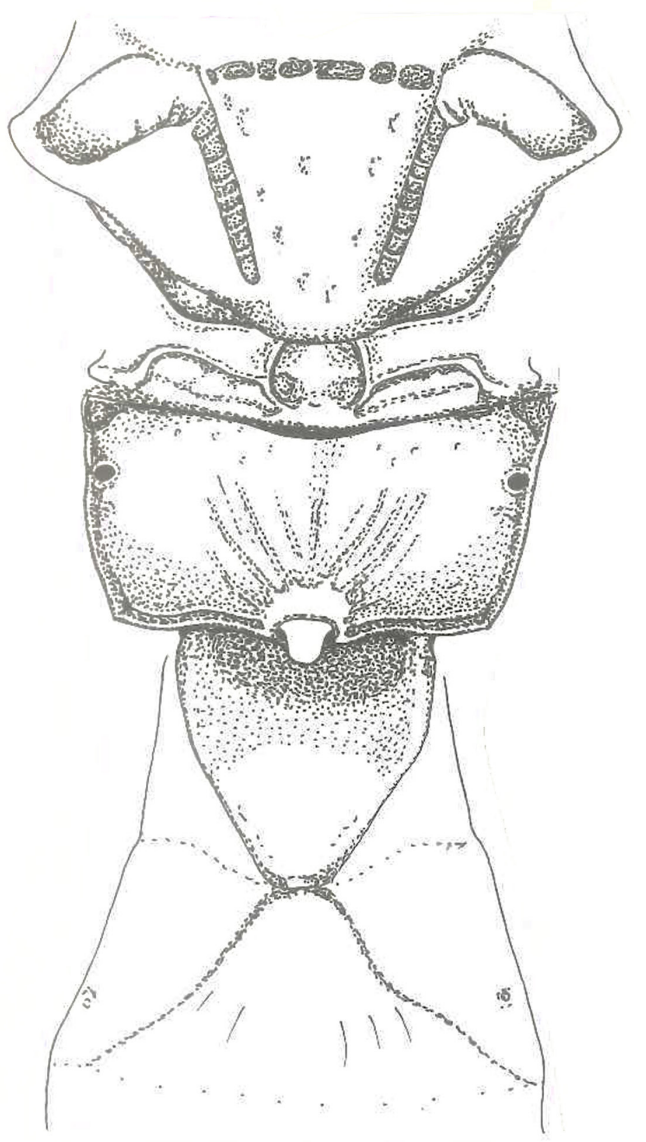

Fig. 16. A-B. Glyptapanteles afiamaluanus (Fullaway, 1941), holotype, $q$ (BPBM) A. Propodeum and T1-2. B. Fore wing. C. G. fullawayi Austin \& Dangerfield, 1992, holotype, ô (BPBM), dorsal scutellum, propodeum and T1-2. D. G. operculinae (Fullaway, 1941), holotype, + (BPBM), dorsal scutellum, propodeum and T1-2. Reproduced from Austin \& Dangerfield (1992). 
All three species also have larger eyes (and consequently, smaller malar spaces) than most other species of Glyptapanteles from Australia. This species group is currently only known from eastern QLD, from Kuranda (northern QLD) and from near Brisbane (southern QLD) (Fig. 4F).

\section{Unplaced species of Glyptapanteles from Australasia}

There are several species of Glyptapanteles from Australasia which we do not place within a species group, either because they do not form a monophyletic group with other species with which they share a clear morphological character, or because they are previously described species without molecular data and/or without clear morphological affinities to any of the assigned species groups. This includes G. afiamaluanus (Fullaway, 1941), G. artonae (Rohwer, 1926), G. aucklandensis (Cameron, 1909), G. baylessi Fagan-Jeffries, Bird \& Austin sp. nov., G. deliasa Austin \& Dangerfield, 1992, G. drioplanetus Fagan-Jeffries \& Austin, 2021, G. fullawayi Austin \& Dangerfield, 1992, G. kurandaensis FaganJeffries, Bird \& Austin sp. nov., G. operculinae (Fullaway, 1941), G. phytometrae (Wilkinson, 1928) and G. taylori (Wilkinson, 1928). Of these unplaced species, only G. baylessi sp. nov., G. deliasa, G. drioplanetus and G. kurandaensis sp. nov. are found in Australia.

\section{Species treatments}

Glyptapanteles afiamaluanus (Fullaway, 1941)

Fig. 16A-B

\section{Diagnosis}

Glyptapanteles afiamaluanus is easily separated from any of the described species of Glyptapanteles in the Australasian region by the distinctive shape of $\mathrm{T} 1$, which broadens significantly in the anterior half and then narrows posteriorly and the strong median carina on the propodeum.

\section{Material examined}

No specimens examined, illustrations in Austin \& Dangerfield (1992: figs 49-50) used as reference (Fig. 16A-B).

\section{Remarks}

Host unknown. This species is very distinctive amongst the Australasian fauna and the genus placement is very tentative.

\section{Distribution}

Samoa (Upolu Is).

Glyptapanteles albigena Fagan-Jeffries, Bird \& Austin sp. nov. urn:1sid:zoobank.org:act:B83B4BAE-3154-4717-A00E-EB6FC9EFF9CD

Figs $5 \mathrm{~A}, 17$

\section{Diagnosis}

Glyptapanteles albigena sp. nov. is in the G. albigena species group and can be separated from other members of the group by the gena having a very large pale spot, covering at least a third of the gena height.

\section{Etymology}

The species epithet 'albigena' is from the Latin adjective 'album' (meaning 'white') and refers to the pale spot on the gena that is characteristic of this species and the broader species group. 


\section{Material examined}

\section{Holotype}

AUSTRALIA • 9 ; New South Wales, Werrikimbe National Park, Plateau Beach Cpg; 31.1807, 152.3266; 1055 m a.s.1.; 17-20 Jan. 2018; K.M. Bayless leg.; sweep ferns along trail; Extraction1110, BOLD: AUGLY048-21; ANIC 32130334.

\section{Paratypes}

AUSTRALIA - Australian Capital Territory - 1 đ̇; Brindabella, Blundells Creek; -35.416667, 148.833333; 8 Jan. 2018; K.M. Bayless leg.; sweep; Extraction1185, BOLD: AUGLY070-21; AM K.379877. - New South Wales • 1 \% ; same collection data as for holotype; Extraction1177, BOLD: AUGLY068-21; ANIC 32 130335 - 1 ; ; same collection data as for holotype; Extraction1178, BOLD: AUGLY069-21; ANIC 32 130336 • 1 \& (ethanol); Monga National Park, near Penance Grove Walk; -35.597372, 149.912126; 9 Nov. 2019-16 Jan. 2020; K.M. Bayless and J.G. Lumbers leg.; Malaise trap over stream, trap destroyed by bushfire; 30 Dec. 2019; partially dried out; Extraction1664, BOLD: AUGLY126-21; ANIC 32 130337 • 1 o (ethanol); Budawang National Park, Mt Budawang Road; -35.4563, 149.9955; 750 m a.s.1.; 16 Jan.-10 Feb. 2019; K.M. Bayless and X. Li leg.; Malaise over Feagans Creek; Extraction1634, BOLD: AUGLY120-21; ANIC 32 130338 • 1 ㅇ; Barren Grounds NR, $21 \mathrm{~m}$ NE of Barren Ground Rd; -34.66971, 150.71167; 1-6 Feb. 2020; K.M. Bayless and J.G. Lumbers leg.; Malaise trap, wet sclerophyll forest; Extraction872, BOLD: AUGLY013-21; ANIC 32 130339 • 1 \%; same collection data as for preceding; Extraction1136, BOLD: AUGLY061-21; ANIC 32130340 • 1 ô; same collection data as for preceding; Extraction871, BOLD: AUGLY012-21; ANIC 32 130341 • 1 Jै; same collection data as for preceding; Extraction1133, BOLD: AUGLY060-21; ANIC 32130342 - 1 (ethanol); same collection data as for preceding; 23-29 Jan. 2020; Extraction1607, BOLD: AUGLY114-21; ANIC 32 130343 - 1 万’; same collection data as preceding; Extraction1609, BOLD: AUGLY115-21; ANIC 32 130344 1 § (ethanol); same collection data as for preceding; Extraction1617, BOLD: AUGLY117-21; ANIC 32 130345. - Queensland - 1 q; Lamington National Park; -28.259, 153.162; 1142 m a.s.1; 11-21 Mar. 2007; C Lambkin and N. Starick leg.; IBISCA Plot \# IQ-1100-B rainforest Malaise trap; Extraction609, BOLD: AUMIC394-18; QM T208405.

\section{Description}

\section{Female}

Colouration. Gena with a pale spot; labrum pale; scape colour in ventral half paler than flagellomeres at proximal end; flagellomeres all black/dark brown; tegula pale; wing veins uniformly black or brown, or with small lighter area proximally; anteromesoscutum all dark, dark with significant orange patches on posterolateral corners or dark with very slight orange patches on posterolateral corners; scutellar disk and metanotum dark; propodeum dark; fore coxa pale yellow; mid coxa pale yellow; hind coxa dark; fore femur pale yellow; mid femur pale yellow; hind femur pale yellow; fore tibia pale yellow; mid tibia pale yellow; hind tibia darkening posteriorly; hind basitarsus light brown; T1 dark or dark reddishbrown; T2 sclerotised area dark or dark reddish-brown; T2 lateral area dark, extends past indentation, but then pale; T3 dark, mostly pale with darker patch in centre or mostly dark with paler lateral areas; T4+ dark or reddish-brown.

Holotype Body MEASUREMENTs. Body length $2.3 \mathrm{~mm}$; fore wing length $2.4 \mathrm{~mm}$; antennal length similar to body length.

HeAD. Antennal flagellomere 14 length/width 1.71-2.16; antennal flagellomere 2 length/width 3.00 3.71; OOD/POD 1.67-1.71; IOD/POD 1.33-1.57.

Mesosoma. Anteromesoscutum sculpturing with shallow to deep punctures, space between punctures a mixture of smaller than diameter of punctures and of similar size. Often smoother in posterior centre 


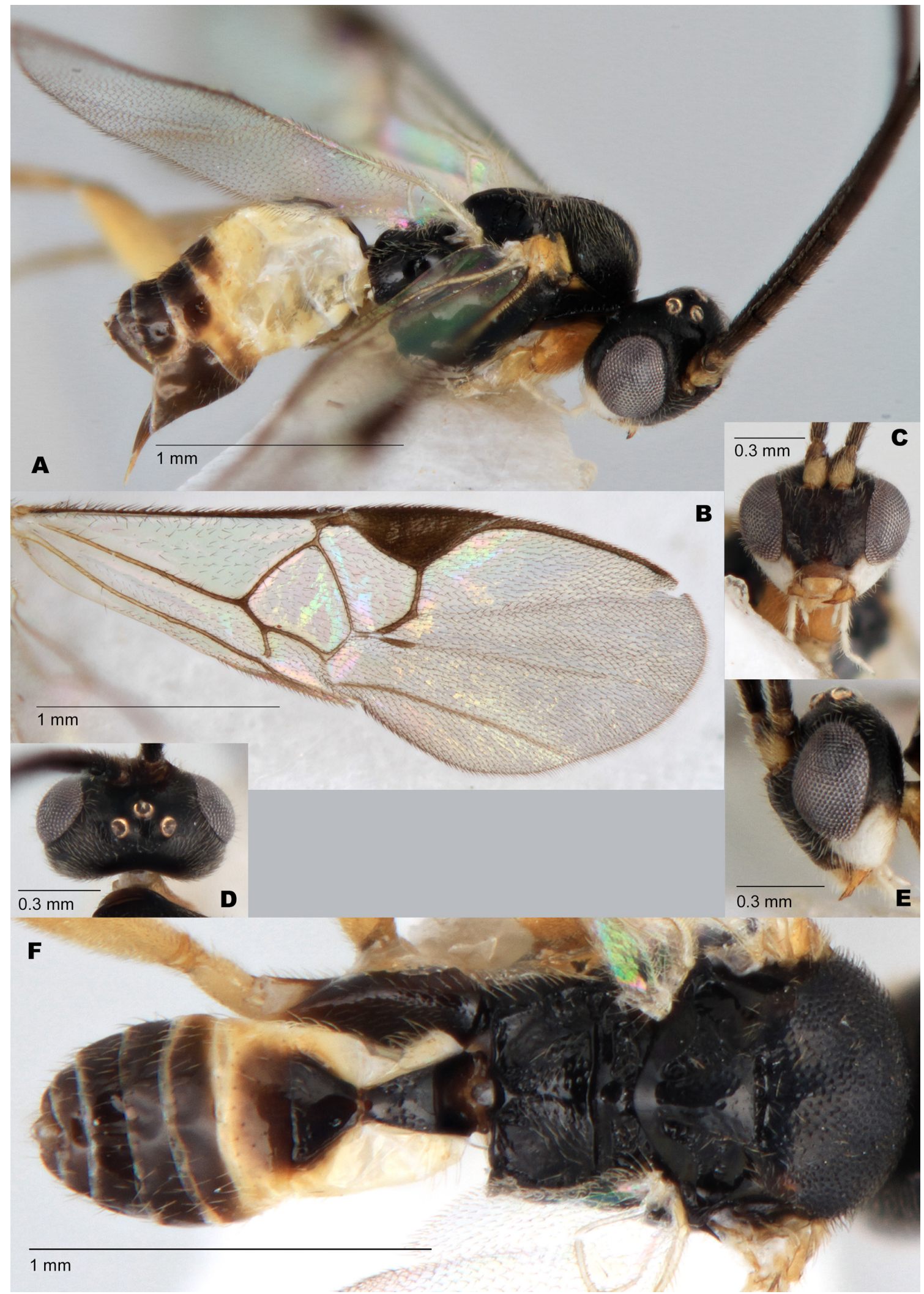

Fig. 17. Glyptapanteles albigena Fagan-Jeffries, Bird \& Austin sp. nov., holotype, q (ANIC 32 130334). A. Lateral habitus. B. Fore wing. C. Anterior head. D. Dorsal head. E. Lateral head. F. Dorsal habitus. 
or shallow punctures, space between punctures generally smaller than diameter of punctures; scutellar disk sculpturing with shallow or very shallow punctures scattered over most of area; $6-8$ pits in the scutellar sulcus; propodeum with median carina faintly indicated for all of length or sometimes only in posterior half; rest of propodeum either smooth with some punctures in anterior half, or smooth with some shallow rugosity, or smooth with both punctures and some shallow rugosity.

Wings. Pterostigma length $0.57 \mathrm{~mm}$; pterostigma width $0.23 \mathrm{~mm} ; \mathrm{r} 0.16 \mathrm{~mm} ; 2 \mathrm{RS} 0.15 \mathrm{~mm} ; 2 \mathrm{~m} 0.14 \mathrm{~mm}$; (RS+M)b $0.09 \mathrm{~mm}$.

Metasoma. T1 wedge-shaped, narrowing posteriorly for entirety of length, lateral edges straight (but not parallel); T1 smooth and shiny, some shallow scattered punctures on lateral edges or smooth and shiny, some shallow scattered punctures on lateral edges and posterior half; T1 length $0.36 \mathrm{~mm}$; T1 width at posterior edge $0.07 \mathrm{~mm}$; 2 an isosceles trapezoid, lateral edges straight; T2 smooth and shiny; T2 length $0.15 \mathrm{~mm}$; T2 width at posterior edge $0.26 \mathrm{~mm}$; ovipositor slightly protruding from end of metasoma.

\section{Male}

As female, but antenna slightly longer than body.

\section{Remarks}

Glyptapanteles albigena sp. nov. constitutes BIN: BOLD:ADL3908 and is 5.48\% (p-dist.) divergent from the closet BIN in the database (BOLD:ABA6208; Glyptapanteles sanniopolus sp. nov.).

Using the BOLD Batch ID engine, the COI barcode of the holotype is $6.3 \%$ different from the most similar COI sequence of an Australian specimen (HYAT425-11 and HYAT424-11; Glyptapanteles sanniopolus sp. nov.). All specimens able to be sequenced for the wingless gene share a unique barcode that differs by a minimum of $1 \mathrm{bp}$ from all other species with available sequence data.

\section{Distribution}

This species is known from the ACT and NSW and from southern QLD.

Glyptapanteles andamookaensis Fagan-Jeffries, Bird \& Austin sp. nov. urn:lsid:zoobank.org:act:B0900129-30DE-49B5-9853-D09AD31602AC

Figs $8 \mathrm{~A}, 18$

\section{Diagnosis}

Glyptapanteles andamookaensis sp. nov. is in the G. albigena species group and can be separated from the other members of the species group by the pale spot on the gena being small but clearly visible, T2 dark, propodeum with the median carina completely absent and hind femur mostly dark (sometimes with a lighter area anteriorly).

\section{Etymology}

Glyptapanteles andamookaensis sp. nov. is named after the collection locality.

\section{Material examined}

Holotype

AUSTRALIA - O; South Australia, Andamooka Station; -30.8198802, 137.1783585 to -30.6998403, 137.1574435; 31 Aug. 2016; R. Leijs leg.; Vehicle net, Bush Blitz Lake Torrens; Extraction260, BOLD: AUMIC130-18; SAMA 32-035451.

\section{Paratype}

AUSTRALIA - 1 q; same collection data as for holotype; Extraction565, BOLD: AUMIC357-18; SAMA 32-035452. 

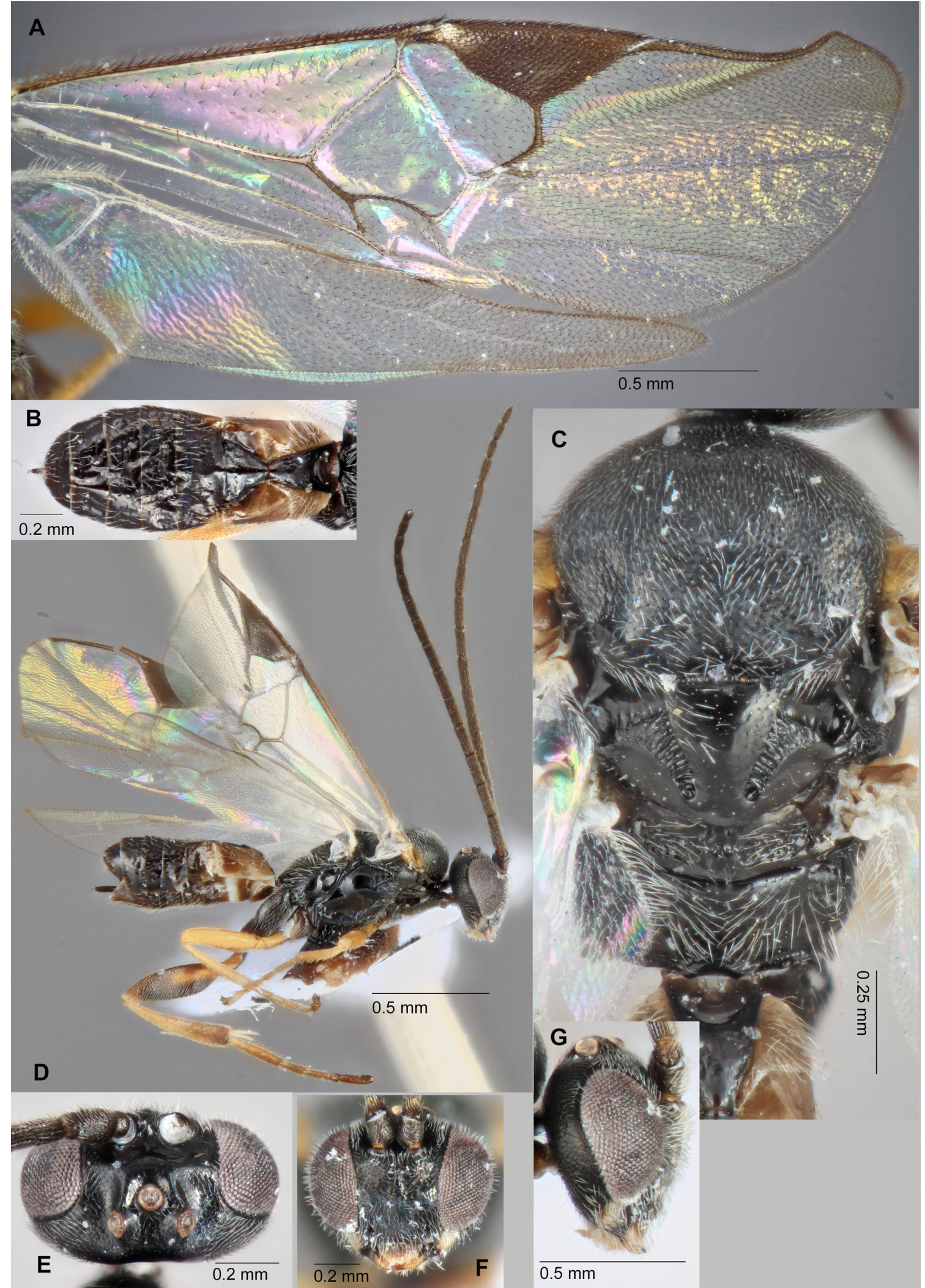

Fig. 18. Glyptapanteles andamookaensis Fagan-Jeffries, Bird \& Austin sp. nov. A-C, F-G. Holotype, $q$ (SAMA 32-035451). D-E. Paratype, q (SAMA 32-035452). A. Fore wing. B. Dorsal metasoma. C. Dorsal mesosoma. D. Lateral habitus. E. Dorsal head. F. Anterior head. G. Lateral head. 


\section{Description}

\section{Female}

Colouration. Gena with a pale spot; labrum reddish-brown; scape colour in ventral half the same colour or darker than flagellomeres; flagellomeres all black/dark brown; tegula pale; wing veins uniformly black or brown, or with small lighter area proximally; anteromesoscutum all dark; scutellar disk and metanotum dark; propodeum dark; fore coxa dark; mid coxa dark; hind coxa dark; fore femur pale yellow; mid femur pale yellow; hind femur dark reddish-brown; fore tibia orange to light brown; mid tibia orange to light brown; hind tibia darkening posteriorly; hind basitarsus light brown; T1 dark; T2 sclerotised area dark, dark area extending past indentation to non-sclerotised area, but T2 lateral area then pale; T3 dark; T4+ dark.

HoLOTYPE BODY MEASUREMENTS. Body length $2.7 \mathrm{~mm}$; fore wing length $2.7 \mathrm{~mm}$; antennal length slightly longer than body length.

HEAD. Antennal flagellomere 14 length/width 2.00; antennal flagellomere 2 length/width 3.57; OOD/ POD 1.25-1.38; IOD/POD 1.88.

Mesosoma. Anteromesoscutum sculpturing with only very shallow punctures, reasonably smooth; scutellar disk sculpturing with only very shallow punctures, smooth and shiny; 10-11 pits in scutellar sulcus; propodeum with median carina absent, only very shallow punctures associated with setae.

WINGs. Pterostigma length $0.71 \mathrm{~mm}$; pterostigma width $0.29 \mathrm{~mm}$; $\mathrm{r} 0.18 \mathrm{~mm} ; 2 \mathrm{RS} 0.15 \mathrm{~mm} ; 2 \mathrm{~m} 0.11 \mathrm{~mm}$; $(\mathrm{RS}+\mathrm{M}) \mathrm{b} 0.13 \mathrm{~mm}$.

Metasoma. T1 wedge-shaped, narrowing posteriorly for entirety of length, lateral edges straight (but not parallel); T1 smooth and shiny; T1 length $0.41 \mathrm{~mm}$; T1 width at posterior edge $0.08 \mathrm{~mm}$; T2 an isosceles trapezoid, lateral edges straight; T2 smooth and shiny; T2 length $0.17 \mathrm{~mm}$; T2 width at posterior edge $0.28 \mathrm{~mm}$; ovipositor slightly protruding from end of metasoma.

\section{Male}

Unknown.

\section{Remarks}

Glyptapanteles andamookaensis sp. nov. constitutes BIN: BOLD:ADL4705 and is $6.41 \%$ (p-dist.) divergent from the closet BIN in the database (BOLD:ADL3094; Glyptapanteles aspersus sp. nov.).

Using the BOLD Batch ID engine, the COI barcode of the holotype is $6.5 \%$ different from the most similar COI sequence from an Australian specimen (AUMIC374-18; Glyptapanteles aspersus sp. nov.). The two available specimens, both sequenced for the wingless gene, share a unique barcode, that differs by a minimum of $1 \mathrm{bp}$ from all other species with available sequence data.

\section{Distribution}

This species is known only from Andamooka Station in the central arid region of SA.

Glyptapanteles arcanus Fagan-Jeffries, Bird \& Austin sp. nov. urn:1sid:zoobank.org:act:FC2D5B3D-1AD2-478C-8920-8706F1BD594F

Fig. 19

\section{Diagnosis}

Glyptapanteles arcanus sp. nov. is in the G. arcanus species group and can be separated from the other members of the species group (other than from G. vergrandiacus sp. nov.) by the propodeum with having 
coarser sculpturing than all but G. lambkinae sp. nov. and G. erucadesolator sp. nov. and with the T1 less strongly sculptured than in G. lambkinae sp. nov. and G. erucadesolator sp. nov. It is noted, however, that all these species (along with G. goodwinnoakes sp. nov., which has T1 smooth) are morphologically very similar and identifications should be made by comparison of DNA barcodes. There was not a morphological character found to easily differentiate $G$. arcanus sp. nov. from $G$. vergrandiacus sp. nov., which are sister lineages in the current phylogeny (Fig. 2), but these two species differ in the wingless barcode by a single base pair and can also be separated by the COI barcodes, which have a $>3 \%$ divergence.

\section{Etymology}

The species epithet 'arcanus' is a Latin adjective meaning 'mysterious' and refers to the cryptic nature of this species' morphology, which has few distinctive features.

\section{Material examined}

\section{Holotype}

AUSTRALIA - + ; Queensland, Lamington National Park; -28.207, 153.137; 560 m a.s.1.; 9-19 Mar. 2007; C. Lambkin and N. Starick leg.; IBISCA Plot \# IQ-500-A rainforest Malaise trap, bulk vial 22028; Extraction1462, BOLD: AUGLY082-21; QM T250938.

\section{Paratype}

AUSTRALIA - 1 क; Queensland, Lamington National Park; -28.155, 153.139; 282 m a.s.1.; 13-23 Jan. 2007; C. Lambkin and N. Starick leg.; IBISCA Plot \# IQ-300-B rainforest Malaise trap; Extraction641, BOLD: AUMIC423-18; QM T208391.

\section{Description}

\section{Female}

Colouration. Gena without a pale spot; labrum reddish-brown; scape colour in ventral half uniformly paler than flagellomeres; flagellomeres all black/dark brown; tegula light brown; wing veins uniformly reddish-brown; anteromesoscutum dark; scutellar disk and metanotum dark with red tinge; propodeum dark; fore coxa dark; mid coxa dark; hind coxa dark; fore femur pale yellow; mid femur light brown; hind femur dark reddish-brown or orange to light brown; fore tibia pale yellow; mid tibia pale yellow; hind tibia darkening posteriorly; hind basitarsus light brown; T1 dark; T2 sclerotised area dark reddishbrown, dark area extending past indentation to non-sclerotised area, but T2 lateral area then pale; T3 mostly dark with paler lateral areas; T4+ reddish-brown.

Holotype Body MEASUREMENTs. Body length $2.3 \mathrm{~mm}$; fore wing length $2.3 \mathrm{~mm}$; antennal length similar to body length.

HEAD. Antennal flagellomere 14 length/width 1.75-2.16; antennal flagellomere 2 length/width 3.573.71; OOD/POD 1.86-2.17; IOD/POD 1.14-1.50.

Mesosoma. Anteromesoscutum sculpturing with shallow to deep punctures, space between punctures a mixture of smaller than diameter of punctures and of similar size, often smoother in posterior centre; scutellar disk sculpturing with shallow punctures scattered over most of area; 8-9 pits in scutellar sulcus. Propodeum with median carina absent, propodeum coarsely rugose.

Wings. Pterostigma length $0.59 \mathrm{~mm}$; pterostigma width $0.12 \mathrm{~mm} ; \mathrm{r} 0.17 \mathrm{~mm} ; 2 \mathrm{RS} 0.12 \mathrm{~mm} ; 2 \mathrm{~m} 0.1 \mathrm{~mm}$; (RS+M)b $0.08 \mathrm{~mm}$. 


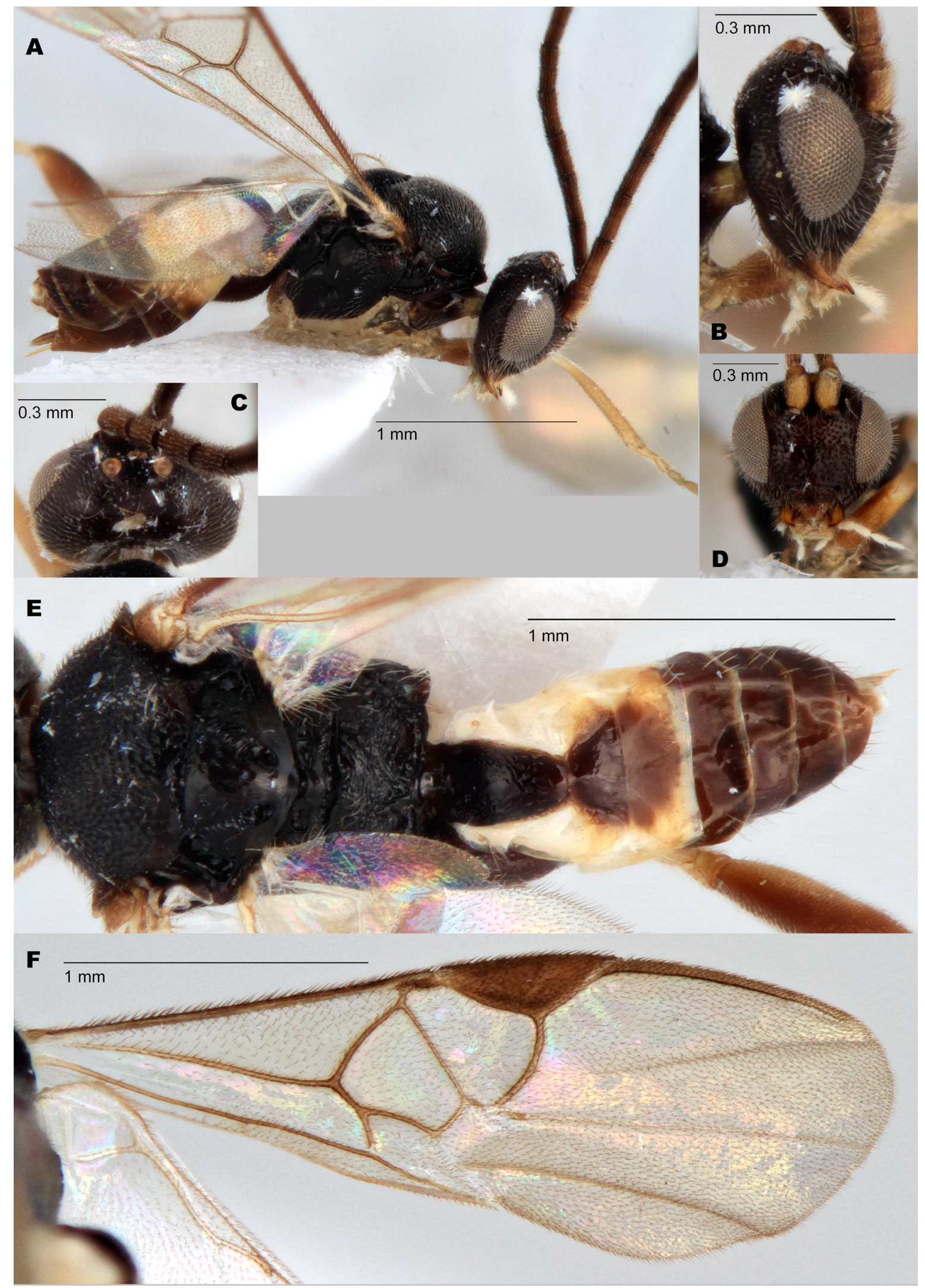

Fig. 19. Glyptapanteles arcanus Fagan-Jeffries, Bird \& Austin sp. nov., paratype, $q$ (QM T208391). A. Lateral habitus. B. Lateral head. C. Dorsal head. D. Anterior head. E. Dorsal habitus. F. Fore wing. 
Metasoma. T1 lateral edges parallel for anterior $1 / 2$ to $2 / 3$ of length, then narrowing posteriorly or broadest at centre of length, narrowing both posteriorly and anteriorly from mid-point; T1 smooth and shiny, some shallow scattered punctures on lateral edges and posterior half or indistinct sculpturing for most of length; T1 length $0.44 \mathrm{~mm}$; T1 width at posterior edge $0.1 \mathrm{~mm}$; T2 an isosceles trapezoid, lateral edges straight; T2 smooth and shiny; T2 length $0.12 \mathrm{~mm}$; T2 width at posterior edge $0.28 \mathrm{~mm}$; ovipositor slightly protruding from end of metasoma.

\section{Male \\ Unknown.}

\section{Remarks}

The wingless barcode for this species is shared with G. doreyi sp. nov. (one haplotype, as there are two wingless haplotypes present within G. doreyi sp. nov.) and G. goodwinnoakes sp. nov. and differs by 1 bp from the barcodes of G. vergrandiacus sp. nov., G. doreyi sp. nov. (second haplotype), and G. lessardi sp. nov. This group of species are very closely related and would benefit from future, more detailed taxonomic work to ensure that they are not simply divergent populations of the same species. For this species hypothesis, we have made the decision to split it into different species based on the COI barcode sequences being $>3 \%$ divergent; however, we note that this is a hypothesis and is open to change with future work.

Glyptapanteles arcanus sp. nov. constitutes BIN BOLD:ADL5262 and is 3.67\% (p-dist.) divergent from the closet BIN in the database (BOLD:ADL3012; Glyptapanteles vergrandiacus sp. nov.).

Using the BOLD Batch ID engine, the COI sequence of the holotype is $3.5 \%$ different from the most similar COI sequence from an Australian specimen (AUGLY102-21; Glyptapanteles vergrandiacus sp. nov.).

\section{Distribution}

This species is known only from Lamington National Park in southern QLD.

\section{Glyptapanteles artonae (Rohwer, 1926)}

\section{Diagnosis}

As Austin \& Dangerfield (1992) state that this species is unlikely to actually occur in the Australasian region and we were unable to find morphological characters that definitively separate this species from those in Australia, we do not diagnose it. However, the extremely dark femur of this species would distinguish it from most new species described in our study. There is a very low risk of this species being conspecific with any of the newly described species from Australia, due to it being unlikely to actually occur in the Australasian region.

\section{Material examined}

\section{Holotype}

MALAYSIA • o; "Kuala Lumpur F.M.S.", "From B.A.R. Gater", "Pars. On Artona catoxantha"; USNM 40097. Only images examined (available at the NMNH type database: http://n2t.net/ark:/65665/30bd958a4-dc6a-446d-8279-4168f0a8e0cc).

\section{Remarks}

See Shenefelt (1972) for a compilation of extra-limital host records (some potentially incorrect). 
Distribution (in the Australasian region)

Fiji (also peninsular Malaysia and Java). Fullaway (1957) is the only record of this species occurring in Fiji. However, Austin \& Dangerfield (1992) were unable to find any such material in world collections and stated that this locality record may be based on a misidentification and the species may not occur in the Australasian region.

Glyptapanteles aspersus Fagan-Jeffries, Bird \& Austin sp. nov. urn:lsid:zoobank.org:act:956B5E0B-3F2A-408A-AAAE-263C197BC3DE

Fig. 20

\section{Diagnosis}

Glyptapanteles aspersus sp. nov. is in the G. albigena species group and can be separated from most members of the species group by the pale spot on the gena being small but clearly visible, T2 dark, the media carina completely absent and the hind femur light brown to pale.

With the limited specimens available, there was not a morphological character found that easily differentiates G. aspersus sp. nov. from G. kittelae sp. nov. or G. austrinus sp. nov. The COI divergence between G. aspersus sp. nov. and G. austrinus sp. nov. is $>7 \%$, whilst the divergence between G. aspersus sp. nov. and G. kittelae sp. nov. is also $>7 \%$, which are considered large divergences at the species level for this group of wasps. The wingless sequences of G. aspersus sp. nov. and G. kittelae sp. nov. are very distinct, differing by 7 bp; however, there are no wingless sequences available for G. austrinus sp. nov.

\section{Etymology}

The species epithet 'aspersus' is an adjective, from the Latin for 'scattered' or 'sprinkled' and refers to the widely dispersed distribution of this species.

\section{Material examined}

\section{Holotype}

AUSTRALIA - ; Western Australia, Watheroo National Park, Jingemia Caves; -30.2542, 115.999; 273 m a.s.l.; 17 Sep.-7 Nov. 2003; C. Lambkin, N. Starick and J. Recsei leg.; Malaise closed heath; Extraction684, BOLD: AUMIC450-18; WAM E109885 (previously ANIC 32 130212).

\section{Paratypes}

AUSTRALIA - South Australia • 1 क; Belair National Park Gate 9; -35.009, 138.654; 25. Nov-1 Dec. 2007; J.T. Jennings leg.; Malaise trap; Extraction59, BOLD: AUMIC374-18; WAM E109886 • 1 क; Bibaringa, Wistow; -35.112, 138.887; Jan.-Feb. 2008; A. Austin leg.; Malaise trap; Extraction73, BOLD: AUMIC482-18; SAMA 32-45045. - Victoria - 1 ô; Mt Macedon; -37.40348, 144.57237; 529 $\mathrm{m}$ a.s.1.; 14 Dec. 2019; J.B. Dorey leg.; general sweep of grass and flowering weeds in open area in schlerophyll, many gums flowering, not overly visited, sunny and overcast $\sim 18^{\circ} \mathrm{C}$; Extraction 1153 , BOLD: AUGLY064-21; SAMA 32-45046.

\section{Description}

\section{Female}

Colouration. Gena with a pale spot; labrum pale or reddish-brown; scape colour in ventral half the same colour or darker than flagellomeres; flagellomeres all black/dark brown; tegula pale; wing veins uniformly black or brown, or with small lighter area proximally or uniformly reddish-brown; anteromesoscutum all dark; scutellar disk and metanotum dark; propodeum dark; fore coxa pale yellow; mid coxa pale yellow; hind coxa dark; fore femur pale yellow or orange to light brown; mid femur pale yellow or orange to light brown; hind femur orange to light brown or dark; fore tibia pale yellow or orange to light 


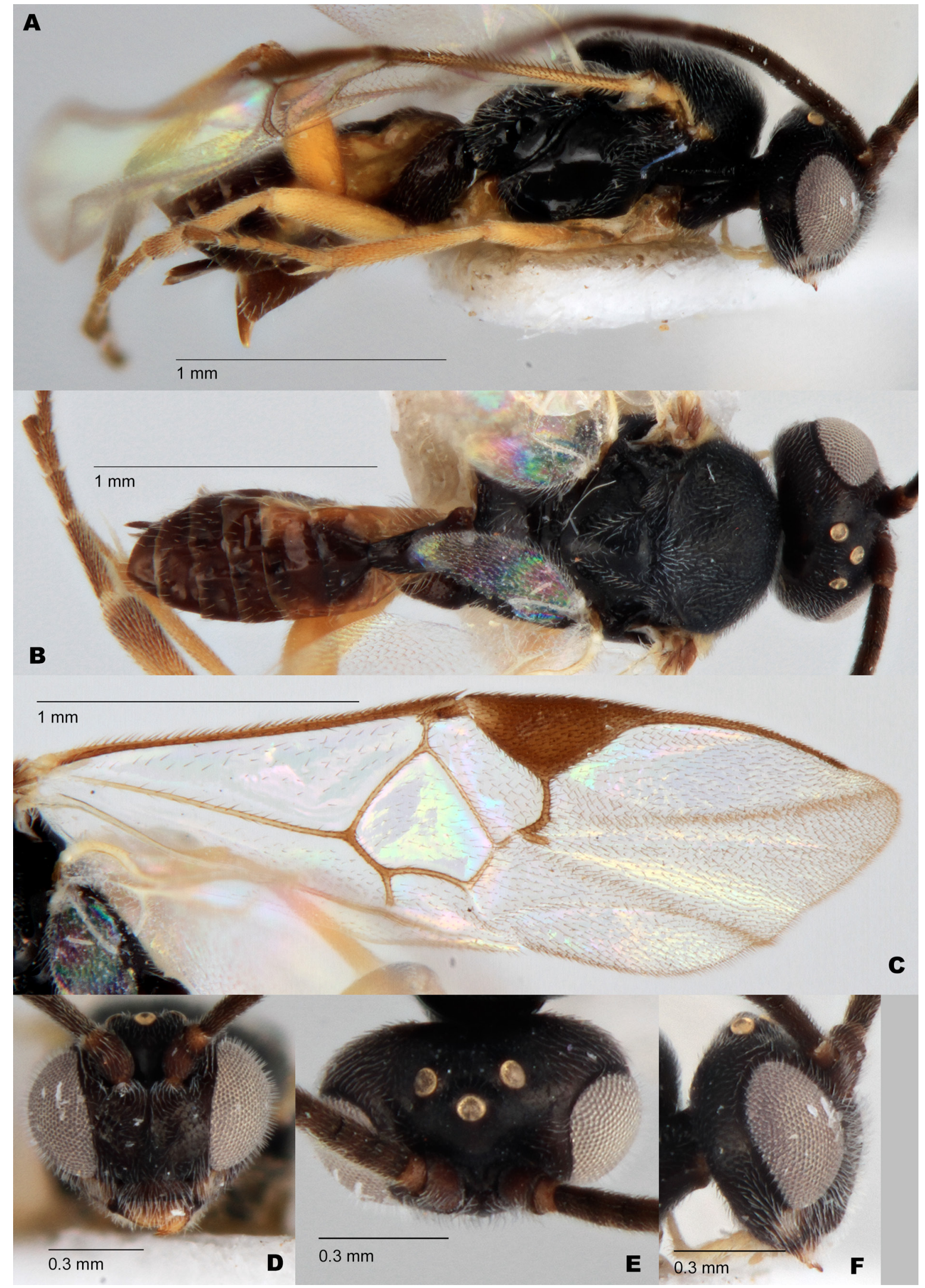

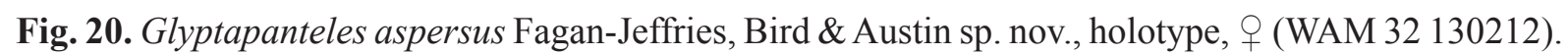
A. Lateral habitus. B. Dorsal habitus. C. Fore wing. D. Anterior head. E. Dorsal head. F. Lateral head. 
brown; mid tibia pale yellow, light brown or orange to light brown; hind tibia darkening posteriorly; hind basitarsus light brown or dark; T1 dark; T2 sclerotised area dark or dark reddish-brown, dark area extending past indentation to non-sclerotised area, but T2 lateral area then pale; T3 dark or mostly dark with paler lateral areas; T4+ dark or reddish-brown.

Holotype Body MEASUREMENTs. Body length $2.2 \mathrm{~mm}$; fore wing length $2.2 \mathrm{~mm}$; antennal length slightly shorter than body length.

HEAD. Antennal flagellomere 14 length/width 2.00-2.20; antennal flagellomere 2 length/width 3.00 4.66; OOD/POD 1.71-2.00; IOD/POD 2.00.

Mesosoma. Anteromesoscutum sculpturing with shallow punctures, space between punctures generally smaller than diameter of punctures; scutellar disk sculpturing with very shallow punctures scattered over most of area; 7-9 pits in scutellar sulcus; propodeum with median carina absent, very smooth and shiny, with either only very shallow punctures associated with setae or punctures over most of area and some shallow rugosity in posterior corners.

WINGS. Pterostigma length $0.54 \mathrm{~mm}$; pterostigma width $0.22 \mathrm{~mm} ; \mathrm{r} 0.14 \mathrm{~mm} ; 2 \mathrm{RS} 0.07 \mathrm{~mm} ; 2 \mathrm{~m} 0.08 \mathrm{~mm}$; $(\mathrm{RS}+\mathrm{M}) \mathrm{b} 0.1 \mathrm{~mm}$.

Metasoma. T1 wedge-shaped, narrowing posteriorly for entirety of length, lateral edges straight (but not parallel); T1 smooth and shiny, some shallow scattered punctures on lateral edges; T1 length $0.38 \mathrm{~mm}$; T1 width at posterior edge $0.08 \mathrm{~mm}$; T2 an isosceles trapezoid, lateral edges straight; T2 smooth and shiny; T2 length $0.13 \mathrm{~mm}$; T2 width at posterior edge $0.27 \mathrm{~mm}$; ovipositor slightly protruding from end of metasoma.

\section{Male}

As female, but antenna slightly longer, six pits in scutellar sulcus.

\section{Remarks}

Glyptapanteles aspersus sp. nov. constitutes BIN: BOLD:ADL3094 and is 5.71\% (p-dist.) divergent from the closet BIN in the database (BOLD:ACL9711; Glyptapanteles sp. from New Zealand).

Using the BOLD Batch ID engine, the COI barcode of the holotype is $6.6 \%$ different from the most similar COI sequence from an Australian specimen (AUMIC021-18; Glyptapanteles harveyi sp. nov.). The holotype was able to be sequenced for the wingless gene, which differs by a minimum of $7 \mathrm{bp}$ from all other species with available sequence data.

\section{Distribution}

This species has a wide distribution, from VIC through to SA across to western WA.

Glyptapanteles aucklandensis (Cameron, 1909)

Fig. 21

\section{Diagnosis}

Glyptapanteles aucklandensis was described from a single male specimen that is not in excellent condition; however, it can be separated from many of the Australian species as follows: from the G. albigena species group by the absence of a pale gena spot, from the mouldsi and eburneus species groups by having T1-2 dark and from the niveus species group by having a relatively smooth anteromesoscutum. With only the one male type available for examination, we cannot conclusively differentiate $G$. aucklandensis from other Australian species, although we note that it has a large number of pits in the scutellar sulcus 

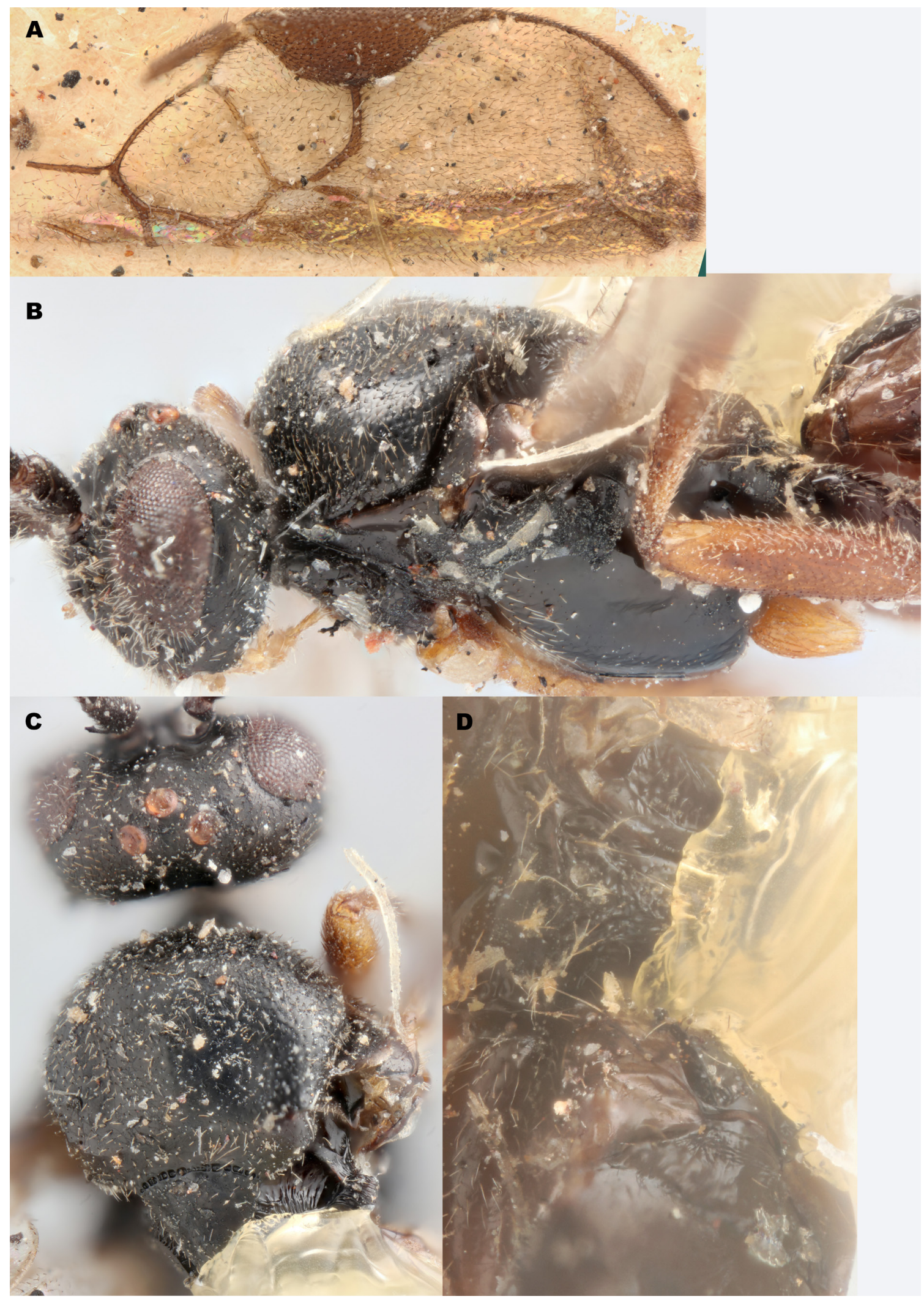

Fig. 21. Glyptapanteles aucklandensis holotype, ô (NHMUK 3.c998). A. Fore wing. B. Lateral head and mesosoma. C. Dorsal head and mesosoma. D. Propodeum and T1-2. Images $\mathbb{C}$ The Trustees of the Natural History Museum, London (CC-BY 4.0). 
$(>16)$ which, if consistent among the rest of the individuals in the species, would separate it easily. There is very little overlap between the Australian and New Zealand microgastrine fauna, with the Glyptapanteles from New Zealand on BOLD forming a distinct clade (Fig. 1), so we feel there is little risk that this species is conspecific with any of the newly described ones from Australia.

\section{Material examined}

Holotype

NEW ZEALAND• đ’; Auckland; “P. Cameron Coll 191?-110”; NHMUK 3.c998 (only images examined).

\section{Remarks}

Host unknown.

\section{Distribution}

New Zealand (Auckland Is).

Glyptapanteles austini Fagan-Jeffries \& Bird sp. nov. urn:1sid:zoobank.org:act:7DAEC136-31EE-4EC2-B197-66F9B686ACEE

Figs 15B, 22

\section{Diagnosis}

Despite the large molecular divergence in both the COI and wingless genes (see Remarks section), the only morphological character found to differentiate G. austini sp. nov. from G. guzikae sp. nov. is the femur being completely dark ( $G$. guzikae sp. nov. with the femur pale with a dark area posteriorly). Glyptapanteles austini sp. nov. can be separated from G. kingae sp. nov. as follows: G. kingae sp. nov. has the propodeum with stronger sculpturing in the centre, whilst G. austini sp. nov. has the propodeum smoother in the centre. However, we note that there are limited specimens available and the variability of propodeal sculpturing could broaden as more populations of these species are identified and we recommend confirming identifications with DNA barcodes.

\section{Etymology}

Named for Professor Andrew Austin, who has championed the documentation of Microgastrinae in Australia and has made a significant impact on the field of systematics of parasitic Hymenoptera, along with providing over a decade of mentorship and training to EPF-J.

\section{Material examined}

\section{Holotype}

AUSTRALIA - + ; South Australia, Mt Barker Summit; -35.0661, 138.923; 20 Mar.-3 Apr. 2016; A. Austin and E. Fagan-Jeffries leg.; Malaise trap; Extraction343, BOLD: AUMIC198-18; SAMA 3245047.

\section{Paratypes}

AUSTRALIA - South Australia - 1 q; same collection data as for holotype; 3-22 Apr. 2016; Extraction340, BOLD: AUMIC196-18; SAMA 32-45048 • 1 क (ethanol); same collection data as for preceding; Extraction210, BOLD: AUMIC095-18; SAMA 32-45049 • 1 \& (ethanol); Belair National Park Gate 11; -35.009, 138.654; 11-24 Nov. 2007; J.T. Jennings leg.; Malaise trap; Extraction355, BOLD: AUMIC208-18; SAMA 32-45050 • 1 क; same collection data as for preceding; 25 Nov.-1 Dec. 2007; Extraction367, BOLD: AUMIC219-18; SAMA 32-46151 • 1 o; Millbrook Reservoir Gate 28, 25 km NE Adelaide; -34.8078, 138.827; 12 Sep. 2007; L. Farrington leg.; Malaise trap; Extraction469, BOLD: AUMIC291-18; SAMA 32-46152. 


\section{Description}

\section{Female}

Colouration. Gena without a pale spot; labrum pale or reddish-brown; scape colour in ventral half the same colour or darker than flagellomeres; flagellomeres all black/dark brown; tegula pale; wing veins uniformly black or brown, or with small lighter area proximally; anteromesoscutum all dark; scutellar disk and metanotum dark; propodeum dark; fore coxa dark; mid coxa dark; hind coxa dark; fore femur orange to light brown; mid femur dark proximally and lightening distally; hind femur dark; fore tibia orange to light brown; mid tibia orange to light brown; hind tibia darkening posteriorly; hind basitarsus dark reddish-brown; T1 dark; T2 sclerotised area dark or dark reddish-brown; T2 lateral area much paler; T3 mostly pale with darker patch in centre or mostly dark with paler lateral areas; T4+ dark.

Body measurements. Body length $2.1 \mathrm{~mm}$; fore wing length $2.0 \mathrm{~mm}$; antennal length slightly shorter than body length.

HEAD. Face with fine punctures associated with setae; antennal flagellomere 14 length/width 1.83-2.50; antennal flagellomere 2 length/width 2.57-3.85; OOD/POD 1.83-2.00; IOD/POD 1.43-2.17.

Mesosoma. Anteromesoscutum sculpturing with shallow punctures, space between punctures generally smaller than diameter of punctures, slightly denser and deeper punctures anteriorly, smoother in posterior centre; scutellar disk sculpturing with only very shallow punctures; 7-9 pits in scutellar sulcus; propodeum with median carina absent, scattered punctures associated with setae in posterior half, rest of propodeum smooth and shiny.

WINGS. Pterostigma length $0.52 \mathrm{~mm}$; pterostigma width $0.18 \mathrm{~mm} ; \mathrm{r} 0.12 \mathrm{~mm} ; 2 \mathrm{RS} 0.11 \mathrm{~mm} ; 2 \mathrm{~m} 0.06 \mathrm{~mm}$; (RS+M)b $0.09 \mathrm{~mm}$.

Metasoma. T1 wedge-shaped, narrowing posteriorly for entirety of length, or lateral edges parallel for anterior $1 / 4$ of length, then gently narrowing posteriorly, lateral edges either straight or with slight curve; T1 mostly smooth, some punctures in posterior half; T1 length $0.3 \mathrm{~mm}$; T1 width at posterior edge $0.09 \mathrm{~mm}$; T2 an isosceles trapezoid, lateral edges straight; T2 smooth and shiny; T2 length $0.11 \mathrm{~mm} ; \mathrm{T} 2$ width at posterior edge $0.19 \mathrm{~mm}$; ovipositor slightly protruding from end of metasoma.

\section{Male \\ Unknown.}

\section{Remarks}

Glyptapanteles austini sp. nov. constitutes BIN BOLD:ADL2587 and is 7.69\% (p-dist.) divergent from the closet BIN in the database (BOLD:ADL2952; Glyptapanteles guzikae sp. nov.).

Using the BOLD Batch ID engine, the COI barcode of the holotype is $7.9 \%$ different from the most similar COI sequence from an Australian specimen (AUMIC524-18; Glyptapanteles guzikae sp. nov.). All five paratype specimens were sequenced for the wingless gene, which is identical amongst all specimens (although there is a single ambiguity in one sequence). The wingless sequences differ by a minimum of $16 \mathrm{bp}$ from all other species with available sequence data.

\section{Distribution}

This species is currently known from SA, from Adelaide and close surrounds. 


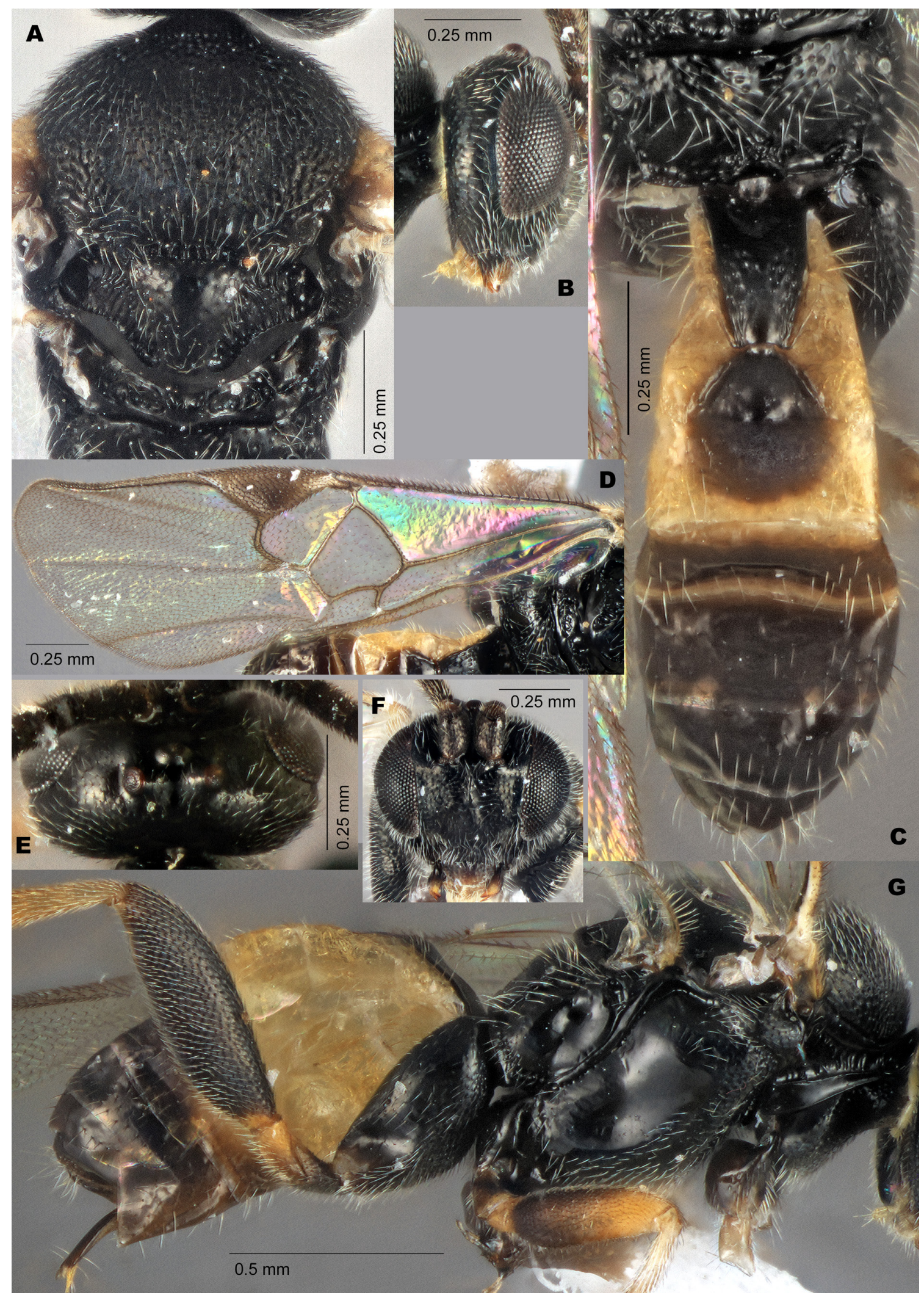

Fig. 22. Glyptapanteles austini Fagan-Jeffries \& Bird sp. nov. A, D-F. Paratype, + (SAMA 32-46151). B-C, G. Holotype, ㅇ (SAMA 32-45047). A. Dorsal mesosoma. B. Lateral head. C. Dorsal metasoma. D. Fore wing. E. Dorsal head. F. Anterior head. G. Lateral habitus. 
Glyptapanteles austrinus Fagan-Jeffries, Bird \& Austin sp. nov. urn:1sid:zoobank.org:act:28E8E832-7CC3-40A0-A29D-D7D799318C95

Fig. 23

\section{Diagnosis}

Glyptapanteles austrinus sp. nov. is in the G. albigena species group and can be separated from most of the other members of the species group by the pale spot on the gena being small but clearly visible, T2 dark, the media carina completely absent, and the hind femur light brown to pale.

With the limited specimens available, there was not a morphological character found that easily differentiates $G$. austrinus sp. nov. from G. kittelae sp. nov. or G. aspersus sp. nov. The COI divergence between G. austrinus sp. nov. and G. kittelae sp. nov. is $>6 \%$, whilst the divergence between G. austrinus sp. nov. and G. aspersus sp. nov. is $>8 \%$, both of which are considerably large divergences at the species level for this subfamily of wasps. There are no available wingless sequences for G. austrinus sp. nov.

\section{Etymology}

The Latin adjective 'austrinus' means 'southern' and refers to the collection of this species near the southern tip of Australia, in Hobart, TAS.

\section{Material examined}

Holotype

AUSTRALIA • O; Tasmania, Hobart, Kingston Beach; -42.986, 147.317; 25-30 Apr. 2010; B. Ward leg.; BIOUG00995-H02, BOLD: HYAT371-11; TMAG F121367.

\section{Paratype}

AUSTRALIA • 1 क ; same collection data as for holotype; BIOUG00996-B04, BOLD: HYAT396-11; TMAG F121368.

\section{Description}

\section{Female}

Colouration. Gena with a pale spot; labrum pale; scape colour in ventral half the same colour or darker than flagellomeres; flagellomeres all black/dark brown; tegula pale; wing veins uniformly black or brown, or with small lighter area proximally; anteromesoscutum all dark; scutellar disk and metanotum dark; propodeum dark; fore coxa pale yellow or dark; mid coxa pale yellow or dark; hind coxa dark; fore femur pale yellow; mid femur pale yellow; hind femur pale yellow; fore tibia pale yellow; mid tibia pale yellow; hind tibia darkening posteriorly; hind basitarsus light brown; T1 dark reddish-brown; T2 sclerotised area dark reddish-brown; T2 lateral area same colour as sclerotised area, or only slightly paler or dark portion extends past indentation, but then pale; $\mathrm{T} 3$ uniformly brown; T4+ reddish-brown.

Holotype Body MEASUREMENTS. Body length $2.3 \mathrm{~mm}$; fore wing length $2.4 \mathrm{~mm}$; antennal length slightly shorter than body length.

HEAD. Antennal flagellomere 14 length/width 1.22-1.50; antennal flagellomere 2 length/width 3.00 4.00; OOD/POD 2.00-2.17; IOD/POD 1.83.

Mesosoma. Anteromesoscutum sculpturing with only very shallow punctures, reasonably smooth; scutellar disk sculpturing with only very shallow punctures, smooth and shiny; 8-11 pits in scutellar sulcus; propodeum with median carina absent, only very shallow punctures associated with setae. 


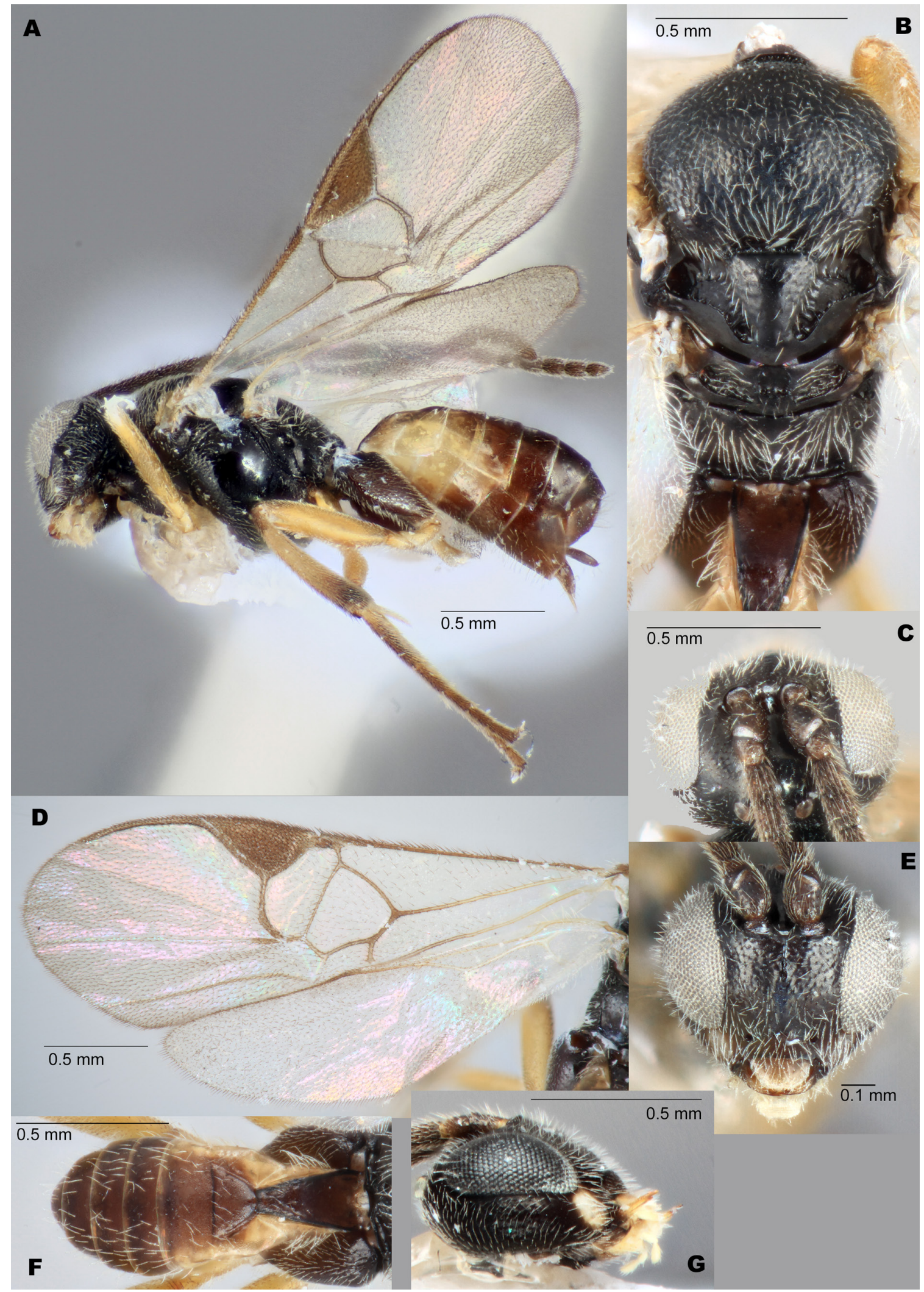

Fig. 23. Glyptapanteles austrinus Fagan-Jeffries, Bird \& Austin sp. nov. A, C-E. Holotype, $q$ (TMAG F121367). B, F-G. Paratype, $q$ (TMAG F121368). A. Lateral habitus. B. Dorsal mesosoma. C. Dorsal head. D. Fore wing. E. Anterior head. F. Dorsal metasoma. G. Lateral head. 
Wings. Pterostigma length $0.57 \mathrm{~mm}$; pterostigma width $0.27 \mathrm{~mm} ; \mathrm{r} 0.17 \mathrm{~mm} ; 2 \mathrm{RS} 0.11 \mathrm{~mm} ; 2 \mathrm{~m} 0.12 \mathrm{~mm}$; $(\mathrm{RS}+\mathrm{M}) \mathrm{b} 0.07 \mathrm{~mm}$.

Metasoma. T1 wedge-shaped, narrowing posteriorly for entirety of length, lateral edges straight (but not parallel); T1 mostly smooth, some punctures in posterior half or smooth and shiny; T1 length $0.34 \mathrm{~mm}$; T1 width at posterior edge $0.07 \mathrm{~mm}$; T2 an isosceles trapezoid, lateral edges straight or with curved lateral and anterior edges, becoming arch- or semicircle-shaped; T2 smooth and shiny; T2 length $0.14 \mathrm{~mm}$; T2 width at posterior edge $0.26 \mathrm{~mm}$; ovipositor slightly protruding from end of metasoma.

\section{Male \\ Unknown.}

\section{Remarks}

Glyptapanteles austrinus sp. nov. constitutes BIN BOLD:ABA6213 and is 4.17\% (p-dist.) divergent from the closet BIN in the database (BOLD:ADL4089; Glyptapanteles ferrugineus sp. nov.).

Using the BOLD Batch ID engine, the COI barcode of the holotype is $4.1 \%$ different from the most similar COI sequence from an Australian specimen (AUMIC042-18; Glyptapanteles ferrugineus sp. nov.). The holotype was able to be sequenced for the wingless gene, which differs by a minimum of $5 \mathrm{bp}$ from all other species with available sequence data.

\section{Distribution}

This species is known only from Hobart in southern TAS.

Glyptapanteles baylessi Fagan-Jeffries, Bird \& Austin sp. nov. urn:lsid:zoobank.org:act:D0EE87F6-0435-407D-AE00-11BBCAF4A184

Figs 5E, 12C, 24

\section{Diagnosis}

Glyptapanteles baylessi sp. nov. is closely related to the G. niveus species group, but can be differentiated by the deep punctures on the anteromesoscutum being denser (much less smooth space between punctures). Glyptapanteles baylessi sp. nov. has strong orange markings on the posterior anteromesoscutum that are significantly larger than in any of the other described species of Glyptapanteles in Australia.

\section{Etymology}

This species is named for Dr Keith Bayless, who collected the type series and also contributed a significant amount of critical additional material used in this study. We thank him for his continual willingness to share Malaise trap samples and his collegiate and open approach to taxonomic science.

\section{Material examined}

\section{Holotype}

AUSTRALIA • $\%$; New South Wales, Royal National Park, near Waterfall Couranga Track near Hacking River; -34.1486, 151.0221; 40 m a.s.1.; 20 Jan.-6 Feb. 2020; K.M. Bayless and J.G. Lumbers leg.; 6 m Malaise trap over Waterfall Creek; Extraction852, BOLD: AUGLY004-21; ANIC 32130346.

\section{Paratypes}

AUSTRALIA - New South Wales 1 ○’; same collection data as for holotype; Extraction859, BOLD: AUGLY007-21; ANIC 32 130347 • 1 万े; same collection data as for preceding; Extraction937, BOLD: AUGLY022-21; ANIC $32130348 \cdot 1$ § ; same collection data as for preceding; Extraction941, BOLD: AUGLY023-21; ANIC 32 130349 • 1 ; ; same collection data as for preceding; Extraction1117, BOLD: 
AUGLY052-21; ANIC 32 130350 1 ते; same collection data as for preceding; Extraction1114, BOLD: AUGLY049-21; ANIC 32 130351 1 ô (ethanol); same collection data as for preceding; Extraction1118, BOLD: AUGLY053-21; ANIC 32 130352 1 क; same collection data as for preceding; Extraction861, BOLD: AUGLY009-21; ANIC 32 130353 • 1 क; same collection data as for preceding; Extraction932, BOLD: AUGLY020-21; ANIC 32 130354 - 1 क; same collection data as for preceding; Extraction1024, BOLD: AUGLY037-21; ANIC 32 130355 1 क; same collection data as for preceding; Extraction1029, BOLD: AUGLY038-21; ANIC 32 130356 • 1 ㅇ (ethanol); same collection data as for preceding; Extraction1116, BOLD: AUGLY051-21; ANIC 32 130357 • 1 ㅇ (ethanol); same collection data as for preceding; Extraction1123, BOLD: AUGLY056-21; ANIC 32 130358 • 1 q (ethanol); same collection data as for preceding; Extraction1127, BOLD: AUGLY058-21; ANIC 32 130359 - 1 ㅇ (ethanol); same collection data as for preceding; Extraction1130, BOLD: AUGLY059-21; ANIC 32130360 • 1 क; Mt Keira via Wollongong; -34.4035, 150.857; 1 Mar. 2004; A.D. Austin and M. Dowton leg.; Extraction43, BOLD: AUMIC264-18; AM K.517936.

\section{Description}

Female

Colouration. Gena without a pale spot; labrum pale; scape colour in ventral half uniformly paler than flagellomeres; flagellomeres all black/dark brown; tegula pale; wing veins uniformly black or brown, or with small lighter area proximally; anteromesoscutum dark with significant orange patches on posterolateral corners; scutellar disk and metanotum dark; propodeum dark; fore coxa pale yellow or white; mid coxa pale yellow or white; hind coxa dark or dark lightening distally; fore femur pale yellow; mid femur pale yellow; hind femur pale yellow or orange to light brown; fore tibia pale yellow; mid tibia pale yellow; hind tibia darkening posteriorly; hind basitarsus light brown, dark reddish-brown or dark; T1 dark; T2 sclerotised area dark; T2 lateral area same colour as sclerotised area, or only slightly paler or dark extends past indentation, but then pale; T3 uniformly brown; T4+ dark or reddish-brown.

Holotype BODY MEASUREMENTs. Body length $2.4 \mathrm{~mm}$; fore wing length $2.4 \mathrm{~mm}$; antennal length slightly longer than body length.

HeaD. Face densely sculptured, punctate reticulate; antennal flagellomere 14 length/width 1.63-2.25; antennal flagellomere 2 length/width 3.5-3.62; OOD/POD 1.13-1.71; IOD/POD 1.29-1.45.

Mesosoma. Anteromesoscutum sculpturing with deep punctures, space between punctures a mixture of smaller than diameter of punctures and of similar size; scutellar disk sculpturing with deep, irregularly spaced punctures; 6-8 pits in scutellar sulcus; propodeum with median carina difficult to discern amongst sculpturing, propodeum strongly rugose, median carina absent and propodeum strongly rugose, or median carina very faintly indicated at posterior and anterior edges, propodeum strongly rugose.

WINGS. Pterostigma length $0.64 \mathrm{~mm}$; pterostigma width $0.18 \mathrm{~mm} ; \mathrm{r} 0.13 \mathrm{~mm} ; 2 \mathrm{RS} 0.11 \mathrm{~mm} ; 2 \mathrm{~m} 0.11 \mathrm{~mm}$; $(\mathrm{RS}+\mathrm{M}) \mathrm{b} 0.08 \mathrm{~mm}$.

Metasoma. T1 lateral edges parallel for anterior $1 / 4$ of length, then gently narrowing posteriorly, lateral edges often with slight curve; T1 smooth in anterior half, punctate reticulate in posterior half or smooth in anterior half, punctate reticulate to rugose in posterior half; T1 length $0.4 \mathrm{~mm}$; T1 width at posterior edge $0.19 \mathrm{~mm}$; T2 width greater than length, rectangular with rounded anterior corners; T2 with some shallow indistinct sculpturing or rugose; T2 length $0.13 \mathrm{~mm}$; T2 width at posterior edge $0.36 \mathrm{~mm}$; ovipositor slightly protruding from end of metasoma.

\section{Male}

As female, 6-9 pits in scutellar sulcus. 


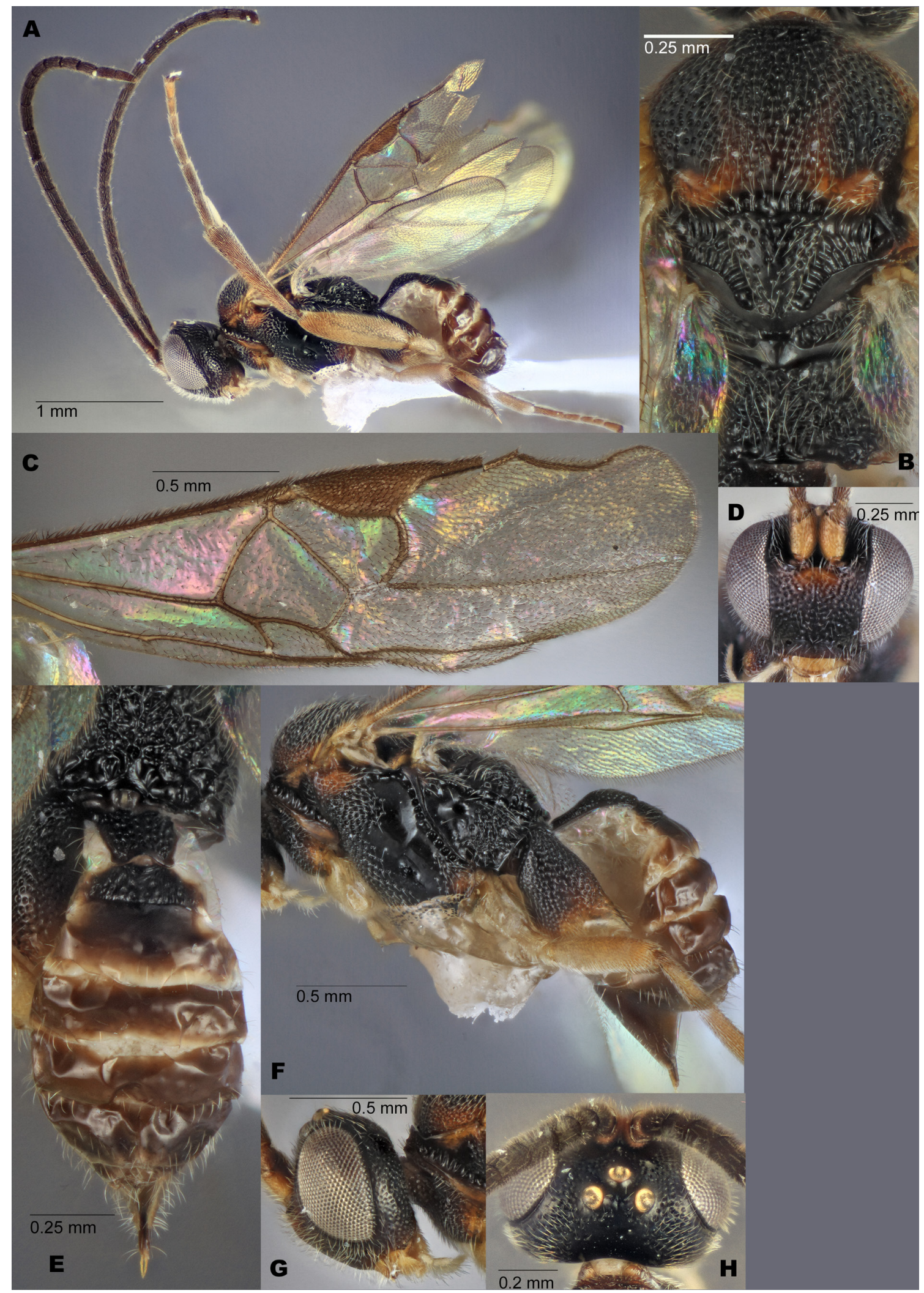

Fig. 24. Glyptapanteles baylessi Fagan-Jeffries, Bird \& Austin sp. nov., paratype, $q$ (AM K.517936). A. Lateral habitus. B. Dorsal mesosoma. C. Fore wing. D. Anterior head. E. Dorsal metasoma. F. Lateral body. G. Lateral head. H. Dorsal head. 


\section{Remarks}

Glyptapanteles baylessi sp. nov. constitutes BIN BOLD:ADL4626 and is 7.98\% (p-dist.) divergent from the closet BIN in the database (BOLD:ADD5918, a BIN consisting of one specimen from Papua New Guinea).

Using the BOLD Batch ID engine, the COI barcode of the holotype is $8.3 \%$ different from the most similar COI sequence from an Australian specimen (AUGLY096-21; Glyptapanteles niveus sp. nov.). Sixteen specimens of the type series were sequenced for the wingless gene, which is identical across all specimens and differs by a minimum of 6 bp from all other species with available sequence data.

\section{Distribution}

This species is currently known from national parks south of Sydney, NSW.

Glyptapanteles bradfordae Fagan-Jeffries, Bird \& Austin sp. nov. urn:lsid:zoobank.org:act:6C1A43F3-D6F0-4FD3-A100-F83B4EB894E8

Figs 12A, 25

\section{Diagnosis}

Glyptapanteles bradfordae sp. nov. is in the G. niveus species group and can be differentiated from G. niveus sp. nov. by the absence of white distal flagellomeres, a pale labrum and denser punctures on the hind coxa. Glyptapanteles bradfordae sp. nov. can be separated from G. cooperi sp. nov. by having denser punctures on the hind coxa, particularly in the dorsal area.

\section{Etymology}

This species is named for Dr Tessa Bradford, Facility Manager of the South Australian Regional Facility for Molecular Ecology and Evolution, without whom the DNA sequencing of many of these specimens would not have occurred. The authors thank Tessa for her continual advice, support and training in the molecular labs!

\section{Material examined}

\section{Holotype}

AUSTRALIA • क; Queensland, Kuranda; -16.8135, 145.6430586; 317 m a.s.1.; 14 Dec. 2019-6 Jan. 2020; M.S. Moulds leg.; Malaise Trap EFJ2020MT11; Extraction1096, BOLD: AUGLY046-21; QM T250939.

\section{Paratypes}

AUSTRALIA 11 ( BOLD: AUGLY078-21; QM T250940 - 1 \% ; same collection data as for holotype; Extraction1297, BOLD: AUGLY079-21; QM T250941 - 1 o (ethanol); same collection data as for holotype; 12 Feb.-6 Apr. 2020; EFJ2020MT36; Extraction1524, BOLD: AUGLY094-21; QM T250942 • 1 入 (ethanol); same collection data as for holotype; 1-21 Jan. 2017; Extraction292, BOLD: AUMIC157-18; QM T250943 - 1 O ; same collection data as for holotype; Extraction290, BOLD: AUMIC155-18; QM T250944 • 1 q; same collection data as for holotype; 12 Feb.-6 Apr. 2020; Extraction1544, BOLD: AUGLY100-21; QM T250945 • 1 ô; Lamington National Park; -28.207, 153.137; 560 m a.s.1.; 9-19 Mar. 2007; C.7 Lambkin and N. Starick leg.; IBISCA Plot \# IQ-500-A rainforest Malaise trap, bulk vial 22028; Extraction1461, BOLD: AUGLY081-21; QM T250946 • 1 \%; same collection data as for preceding; Extraction633, BOLD: AUMIC417-18; QM T208409. 


\section{Description}

\section{Female}

Colouration. Gena without a pale spot; labrum pale; scape colour in ventral half uniformly paler than flagellomeres or the same colour or darker than flagellomeres; flagellomeres all black/dark brown or uniformly reddish-brown; tegula pale; wing veins uniformly black or brown, or with small lighter area proximally; anteromesoscutum dark with significant orange patches on posterolateral corners; scutellar disk and metanotum dark or dark with red tinge; propodeum dark; fore coxa white; mid coxa white; hind coxa dark; fore femur pale yellow; mid femur pale yellow; hind femur pale yellow; fore tibia pale yellow; mid tibia pale yellow; hind tibia darkening posteriorly; hind basitarsus light brown; T1 dark or dark reddish-brown; T2 sclerotised area dark or dark reddish-brown; T2 lateral area much paler; T3 dark or mostly dark with paler lateral areas; T4+ dark or reddish-brown.

Holotype Body meAsurements. Body length $1.8 \mathrm{~mm}$; fore wing length $2.3 \mathrm{~mm}$; antennal length slightly longer than body length.

HEAD. Face densely sculptured, punctate reticulate; antennal flagellomere 14 length/width 1.85-2.00; antennal flagellomere 2 length/width 3.63-4.50; OOD/POD 1.33-1.71; IOD/POD 1.33-1.71. Eyes particularly large, with a smaller malar space than most species of Glyptapanteles.

Mesosoma. Anteromesoscutum sculpturing with shallow punctures, space between punctures generally smaller than diameter of punctures, slightly denser and deeper punctures anteriorly, smoother in posterior centre or very sparse, deep punctures; scutellar disk sculpturing smooth in centre, with some deep punctures on lateral edges; six pits in scutellar sulcus; propodeum with median carina very faintly indicated at either anterior or both posterior and anterior edges, propodeum strongly rugose.

WINGs. Pterostigma length $0.57 \mathrm{~mm}$; pterostigma width $0.18 \mathrm{~mm} ; \mathrm{r} 0.2 \mathrm{~mm} ; 2 \mathrm{RS} 0.1 \mathrm{~mm} ; 2 \mathrm{~m} 0.12 \mathrm{~mm}$; $(\mathrm{RS}+\mathrm{M}) \mathrm{b} 0.09 \mathrm{~mm}$.

Metasoma. T1 lateral edges parallel for anterior $3 / 4$ of length, then narrowing posteriorly or broadest at centre of length, narrowing both posteriorly and anteriorly from mid-point; T1 smooth in anterior half, punctate reticulate to rugose in posterior half; T1 length $0.38 \mathrm{~mm}$; T1 width at posterior edge $0.13 \mathrm{~mm}$; $\mathrm{T} 2$ an isosceles trapezoid, lateral edges straight or almost square, lateral edges almost parallel, only broadening posteriorly very slightly; T2 with some shallow indistinct sculpturing; T2 length $0.12 \mathrm{~mm}$; T2 width at posterior edge $0.22 \mathrm{~mm}$; ovipositor slightly protruding from end of metasoma.

\section{Male}

As female, 6-7 pits in scutellar sulcus.

\section{Remarks}

Glyptapanteles bradfordae sp. nov. constitutes BIN BOLD:AEI7940 and is 2.01\% (p-dist.) divergent from the closet BIN in the database (BOLD:ADD8337, with three specimens from Papua New Guinea, see below).

Using the BOLD Batch ID engine, the COI barcode of the holotype is $7.6 \%$ different from the most similar COI sequence from an Australian specimen (GMAQJ131-17; an undescribed lineage, with two specimens). Eight specimens were able to be sequenced for the wingless gene, which all share a unique barcode that differs by a minimum of $3 \mathrm{bp}$ from all other species with available sequence data.

There are three additional specimens sequenced for $C O I$ on BOLD which fall within $2 \%$ pairwise divergence and are in the closely related BIN BOLD:ADD8337. These specimens (GMNGH687-16, 


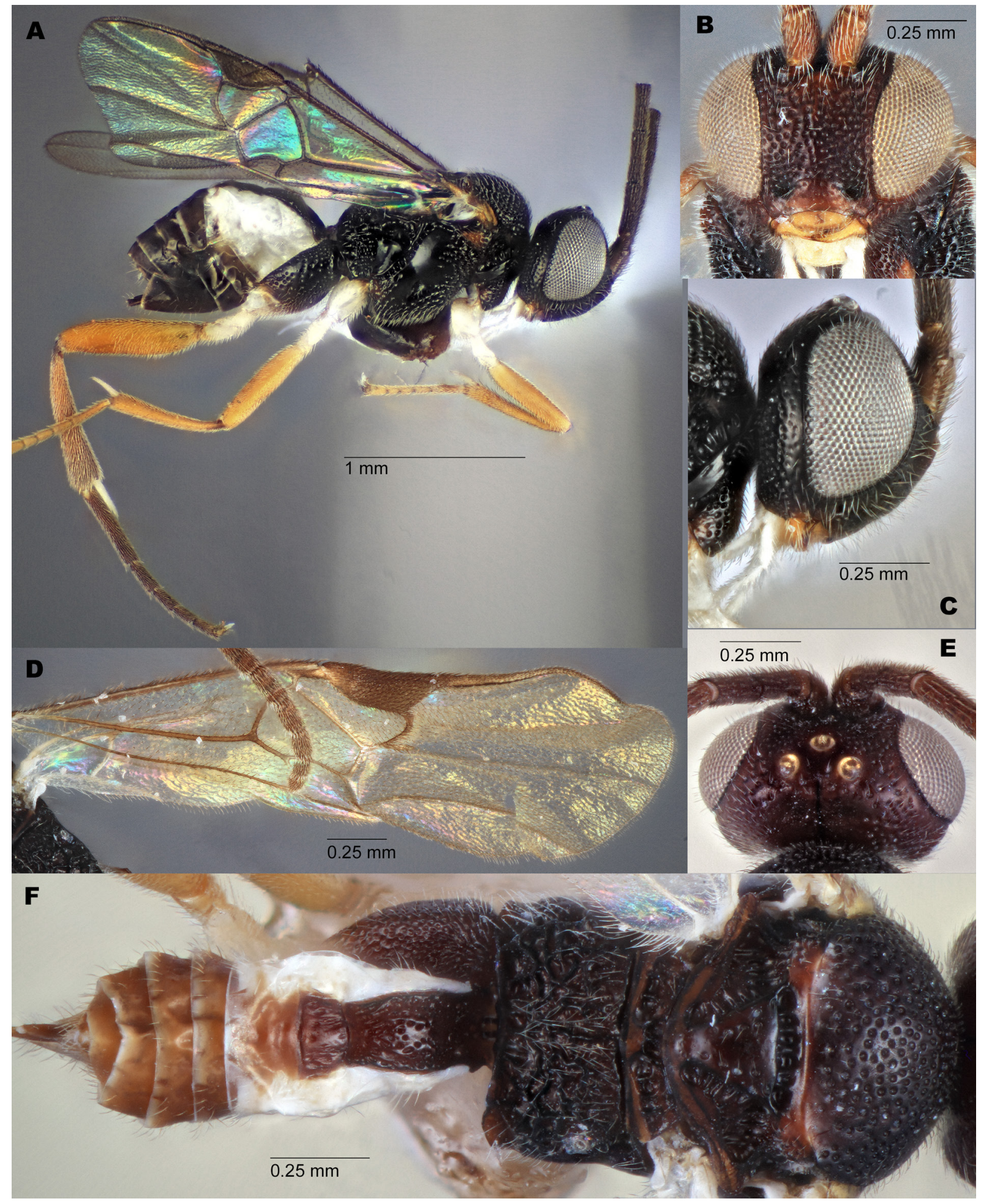

Fig. 25. Glyptapanteles bradfordae Fagan-Jeffries, Bird \& Austin sp. nov., paratypes, †. A, C-D, F. QM T250944. B, E. QM T208409. A. Lateral habitus. B. Anterior head. C. Lateral head. D. Fore wing. E. Dorsal head. F. Dorsal habitus. 
GMNGJ510-16 and GMNGS061-16) were all collected in Papua New Guinea, Madang, by Pagi Toko and are most likely the same species. Specimens were not examined, but a dorsal habitus image on BOLD (http://www.boldsystems.org/pics/GMNGS/BIOUG30657-C01\%2B1487790120.jpg) supports this suggestion.

\section{Distribution}

This species is currently known from the east coast of QLD, from both Lamington National Park in the south and Kuranda in the north. It is also likely found in Papua New Guinea (based on the presence of closely related COI barcodes on BOLD).

Glyptapanteles cooperi Fagan-Jeffries, Bird \& Austin sp. nov. urn:lsid:zoobank.org:act:8E2A7ED6-8383-43C1-B1DA-2712F7B6529C

Figs 12B, 26

\section{Diagnosis}

Glyptapanteles cooperi sp. nov. is in the G. niveus species group and can be separated from G. bradfordae sp. nov. by the hind coxa of $G$. cooperi sp. nov. only being sparsely punctured, particularly on the dorsal area and from G. niveus sp. nov. by the labrum being pale in colour (labrum dark in G. niveus sp. nov.).

\section{Etymology}

Named for Professor Steven Cooper, who has provided extensive mentoring to EPF-J during her PhD.

\section{Material examined}

\section{Holotype}

AUSTRALIA • + ; Queensland, Kuranda; -16.8154, 145.643; 317 m a.s.1.; 16 Mar.-12 Apr. 2017; M.S. Moulds leg.; Malaise Trap; Extraction548, BOLD: AUMIC340-18; QM T250947.

\section{Description}

\section{Female}

COLOURATION. Gena without a pale spot; labrum pale; scape colour in ventral half paler than flagellomeres at proximal end; flagellomeres all black/dark brown; tegula dark; wing veins uniformly black or brown, or with small lighter area proximally; anteromesoscutum dark with very slight orange patches on posterolateral corners; scutellar disk and metanotum dark; propodeum dark; fore coxa white; mid coxa white; hind coxa dark; hind femur orange to light brown; hind tibia light brown; hind basitarsus light brown; T1 dark; T2 sclerotised area dark; T2 lateral area same colour as sclerotised area, or only slightly paler; T3 mostly dark with paler lateral areas; T4+ dark.

Holotype BOdy MEASUREMENTS. Body length $2.3 \mathrm{~mm}$; fore wing length $2.2 \mathrm{~mm}$.

HEAD. Face densely sculptured, punctate reticulate; antennal flagellomere 2 length/width 3.63; OOD/ POD 1.50; IOD/POD 1.50.

Mesosoma. Anteromesoscutum with very sparse, deep punctures; scutellar disk smooth in centre, some deep punctures on lateral edges; six pits in scutellar sulcus; propodeum with median carina present and complete, rest of propodeum strongly rugose.

WINGS. Pterostigma length $0.58 \mathrm{~mm}$; pterostigma width $0.17 \mathrm{~mm} ; \mathrm{r} 0.15 \mathrm{~mm} ; 2 \mathrm{RS} 0.1 \mathrm{~mm} ; 2 \mathrm{~m} 0.11 \mathrm{~mm}$; $(\mathrm{RS}+\mathrm{M}) \mathrm{b} 0.08 \mathrm{~mm}$. 


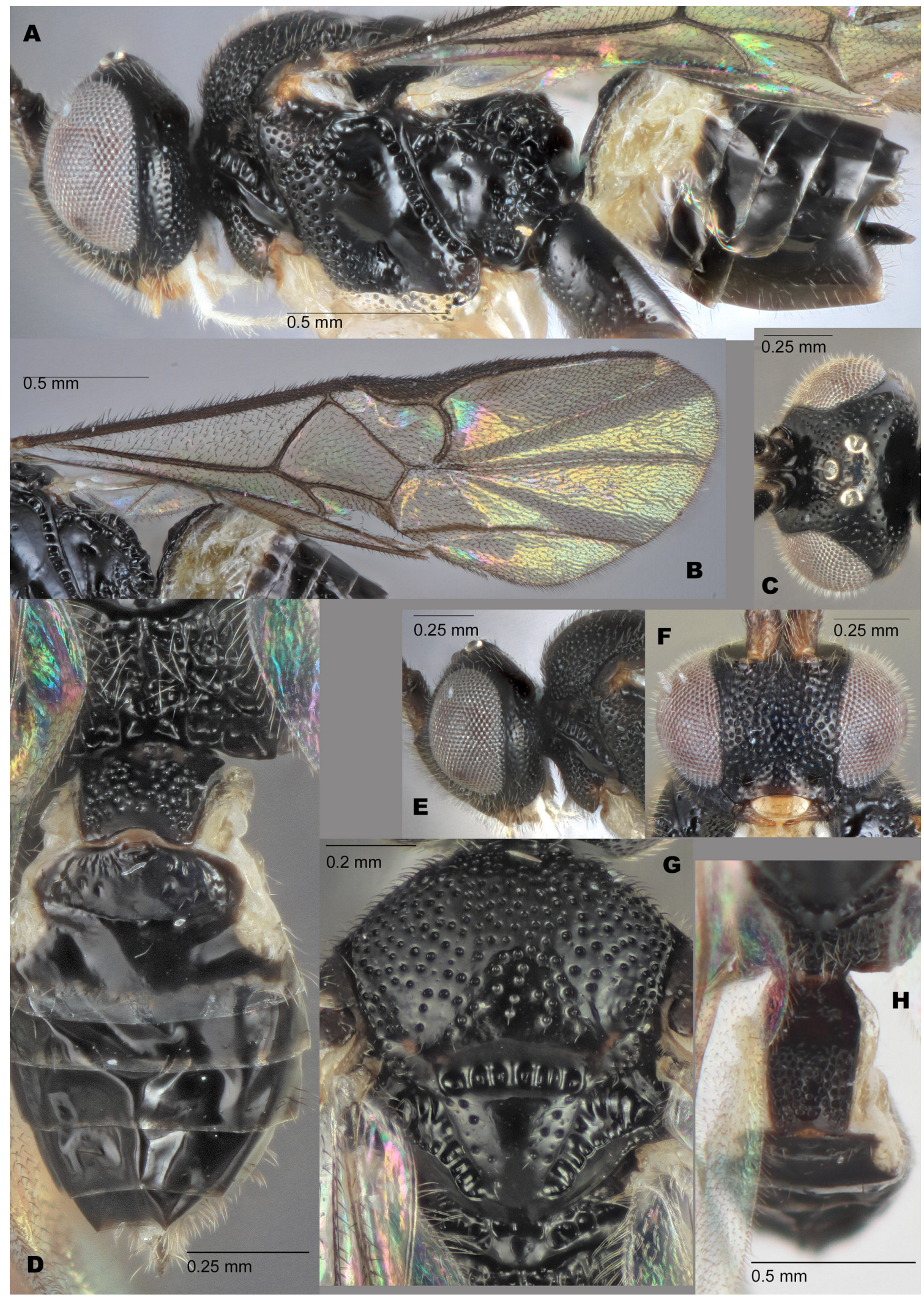

Fig. 26. Glyptapanteles cooperi Fagan-Jeffries, Bird \& Austin sp. nov., holotype, $q$ (QM T250947). A. Lateral body. B. Fore wing. C. Dorsal head. D. Dorsal propodeum and metasoma. E. Lateral head. F. Anterior head. G. Dorsal mesosoma. H. Dorsal metasoma. 
Metasoma. T1 lateral edges parallel for anterior $1 / 4$ of length, then gently narrowing posteriorly, lateral edges often with slight curve; T1 smooth in anterior half, punctate reticulate in posterior half; T1 length $0.5 \mathrm{~mm}$; T1 width at posterior edge $0.21 \mathrm{~mm}$; T2 width greater than length, rectangular with rounded anterior corners; T2 smooth and shiny; T2 length $0.15 \mathrm{~mm}$; T2 width at posterior edge $0.36 \mathrm{~mm}$; ovipositor slightly protruding from end of metasoma.

\section{Male \\ Unknown.}

\section{Remarks}

Glyptapanteles cooperi sp. nov. constitutes BIN: BOLD:ADL4822 and is $6.98 \%$ (p-dist.) divergent from the closet BIN in the database (BOLD:ADG5990, an undescribed lineage from Australia, with two specimens).

Using the BOLD Batch ID engine, the COI barcode of the holotype is $6.8 \%$ different from the most similar COI sequence from an Australian specimen (GMQQW352-18; an undescribed lineage, with two specimens). The type specimen was able to be sequenced for the wingless gene, which differs by a minimum of $4 \mathrm{bp}$ from all other species with available sequence data.

\section{Distribution}

This species is currently only represented by a single specimen from Kuranda, northern QLD.

Glyptapanteles deliasa Austin \& Dangerfield, 1992

Figs 13A, 27

\section{Diagnosis}

Glyptapanteles deliasa can be separated from the other described species of Glyptapanteles in Australia by the combination of the following characters: gena without a pale spot, T1 and T2 dark, anteromesoscutum very smooth, with only shallow and regular punctures, no orange markings on the postero-lateral anteromesoscutum, propodeum very smooth with only shallow punctures and the median carina faintly indicated, T1 parallel-sided for most of length, only narrowing/curving inwards right at boundary with T3, ventral side of antennal scape (at least in distal half) darker than flagellomeres, tegula dark.

\section{Material examined}

\section{Paratypes}

AUSTRALIA • 2 우; South Australia, Waikerie; 22 Jun. 1988; M.D. Moore leg.; ex Delias aganippe (Donovan) (Pieridae); WINC.

\section{Remarks}

There is a possibility that this species is conspecific with Cotesia deliadis (Bingham, 1906), as it is morphologically very similar and parasitises the same host. However, several attempts to extract DNA from paratype specimens to either confirm or refute this hypothesis have been unsuccessful. As such, we leave it within the genus Glyptapanteles until next-generation sequencing techniques can be used on the type series.

\section{Distribution}

Known from Australia, currently only from SA. 


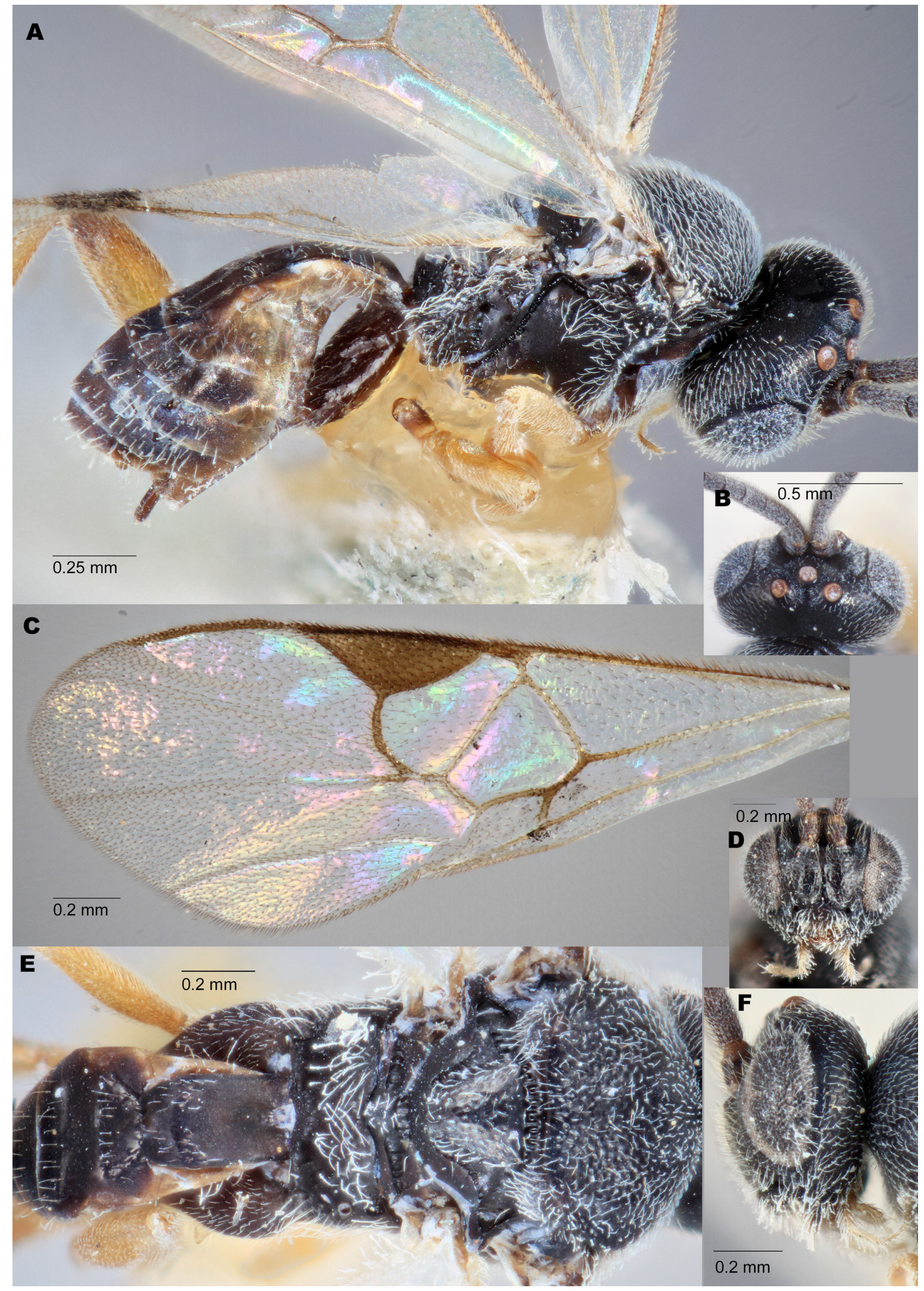

Fig. 27. Glyptapanteles deliasa Austin \& Dangerfield, 1992, paratype, $q$ (WINC). A. Lateral habitus. B. Dorsal head. C. Fore wing. D. Anterior head. E. Dorsal habitus. F. Lateral head. 
Glyptapanteles doreyi Fagan-Jeffries, Bird \& Austin sp. nov. urn:1sid:zoobank.org:act:34246DE7-8966-4FBC-8D6A-ECC5DADF79BC

Figs $28-29$

\section{Diagnosis}

Glyptapanteles doreyi sp. nov. is in the G. arcanus species group and can be separated from the other members of the species group as follows:

Glyptapanteles doreyi sp. nov. can be separated from G. rodriguezae sp. nov. and G. ruhri sp. nov. by T1 being smooth and shiny, not having punctures that cover at least a third of the area of the posterior half of the tergite.

Glyptapanteles doreyi sp. nov. can be separated from G. goodwinnoakes sp. nov., G. erucadesolator sp. nov., G. lambkinae sp. nov., G. arcanus sp. nov. and G. vergrandiacus sp. nov. by the propodeum being less coarsely and less consistently, rugose sculptured across the anterior half of the propodeum. Glyptapanteles doreyi sp. nov. has the propodeum with shallow or strong punctures in the anterior half, the posterior half smooth or with shallow or strong rugose sculpturing and sometimes with a smooth area in the centre.

Glyptapanteles doreyi sp. nov. can be separated from G. wrightae sp. nov. and G. lessardi sp. nov. by the tegula being dark in colouration, the same colour or only slightly lighter than the mesosoma (the tegula in G. wrightae sp. nov. and G. lessardi sp. nov. is pale) the hind femur mostly dark (pale or light brown in G. wrightae sp. nov. and G. lessardi sp. nov.) and the indentation in the centre of the mesopleuron being strongly canaliculate. The indentation on the mesopleuron of G. wrightae sp. nov. and G. lessardi sp. nov. is smoother, not strongly canaliculate.

\section{Etymology}

Named for James Dorey, who collected the holotype specimen. EPF-J would like to acknowledge James' collegiate sharing of specimens and his contribution to a successful field trip in northern QLD in 2019 that led to the collection of many important microgastrine specimens.

\section{Material examined}

\section{Holotype}

AUSTRALIA - O; New South Wales, Cockburn River Camp; -31.05222, 151.14411; 461 m a.s.1.; 10 Dec. 2019; J.B. Dorey leg.; 19JDEC100, general sweep over Brachychiton flowering species in schlerophyll forest along dry creek bed, sunny and warm $\sim 33^{\circ} \mathrm{C}$; Extraction1067, BOLD: AUGLY041-21; AM K.517929.

\section{Paratypes}

AUSTRALIA - Australian Capital Territory - 1 \%; CSIRO Black Mountain off Frith Rd; -35.268, 149.1107; 610 m a.s.1; 9-26 Oct. 2019; K.M. Bayless leg.; dry sclerophyll forest Malaise trap; Extraction1660, BOLD: AUGLY125-21; ANIC 32 130318・1 ; ; same collection data as for preceding; Extraction1645, BOLD: AUGLY122-21; ANIC 32 130319・1 ; ; same collection data as for preceding; Extraction1646, BOLD: AUGLY123-21; ANIC 32 130320 • 1 क (ethanol); CSIRO Black Mountain, close to Botanic Garden fence; -35.273611, 149.110556; 538 m a.s.1.; 30 Oct.-6 Nov. 2017; T. Pleines and J. Rodriguez leg.; Malaise; Extraction959, BOLD: AUGLY027-21; ANIC 32130321 - 1 o ; same collection data as for preceding; 23-30 Oct. 2017; Extraction974, BOLD: AUGLY028-21; ANIC $32130322 \cdot 1$ + (ethanol); CSIRO Black Mountain; -35.2744, 149.1115; 6 Dec. 2017-5 Jan. 2018; J. Rodriguez, C. Waichert, K.M. Bayless and T. Pleines leg.; Malaise 2, green dry wash; Extraction976, BOLD: AUGLY029-21; ANIC 32 130323 - 1 ㅇ (ethanol); same collection data as for preceding; Extraction978, BOLD: AUGLY030-21; ANIC 32 130324• 1 \%; same collection data as for preceding; 


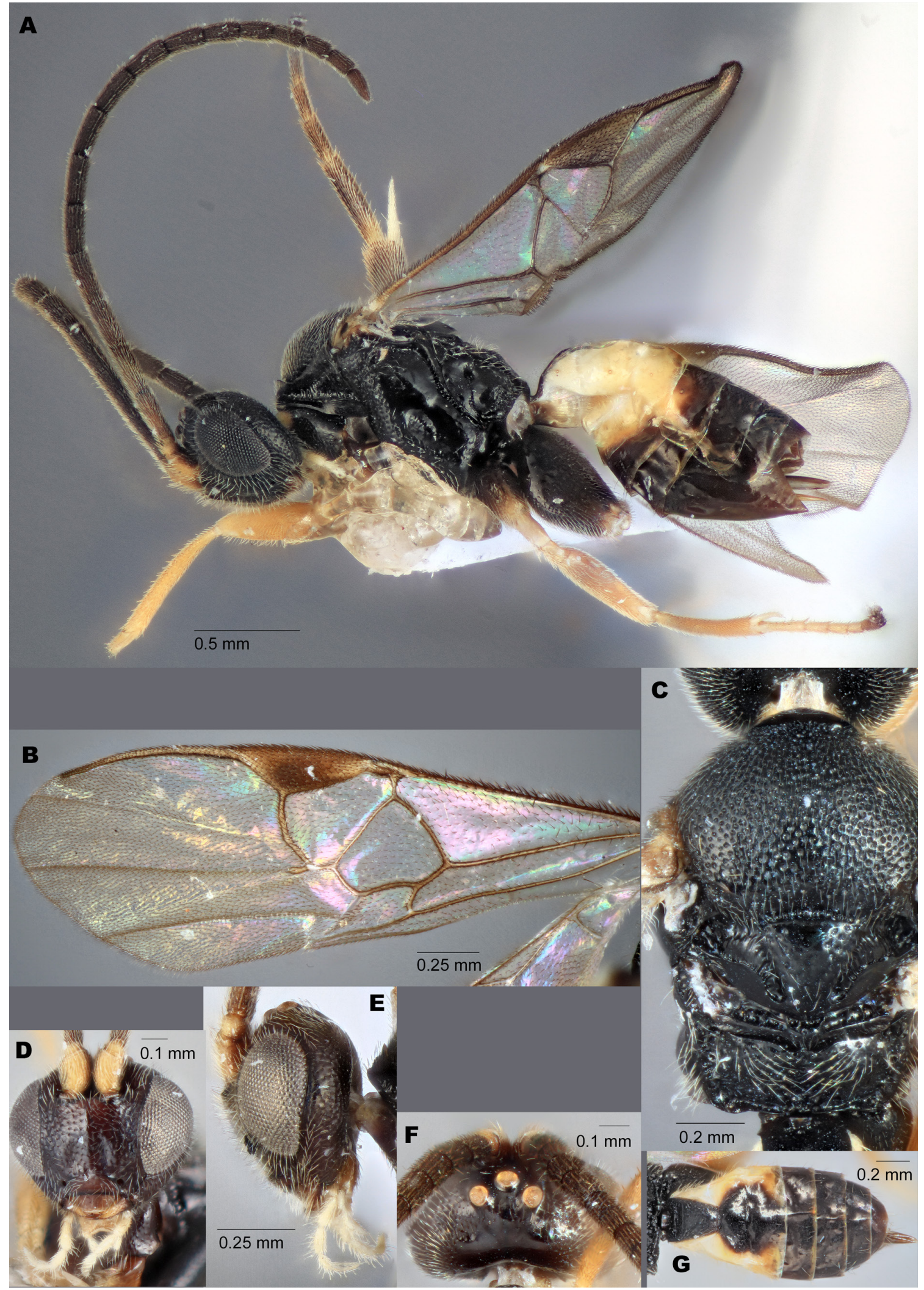

Fig. 28. Glyptapanteles doreyi Fagan-Jeffries, Bird \& Austin sp. nov., paratypes, + , 'clade A'. A, F-G. AUMIC412-18. B-E. AUMIC389-18. A. Lateral habitus. B. Fore wing. C. Dorsal mesosoma. D. Anterior head. E. Lateral head. F. Dorsal head. G. Dorsal metasoma. 
Extraction980, BOLD: AUGLY032-21; ANIC 32 130325 • 1 q (ethanol); same collection data as for preceding; Extraction981, BOLD: AUGLY033-21; ANIC 32 130326 • 1 ㅇ (ethanol); same collection data as for preceding; Extraction982, BOLD: AUGLY034-21; ANIC 32130327 - 1 ㅇ (ethanol); same collection data as for preceding; Extraction983, BOLD: AUGLY035-21; ANIC 32 130328 1 q (ethanol); same collection data as for preceding; 6-20 Jan. 2020; Extraction1658, BOLD: AUGLY124-21; ANIC 32 130329 • 1 क; CSIRO property; -35.275, 149.111; 588 m a.s.1.; 7 Sep. 2011; P. Hebert leg.; Malaise; BIOUG02156-H03, BOLD: MCCAA225-12; ANIC 32 130330. - New South Wales • 1 + (ethanol); same collection data as for holotype; Extraction1086, BOLD: AUGLY043-21; AM K.383784 • 1 o (ethanol); same collection data as for holotype; Extraction1156, BOLD: AUGLY066-21; AM K.383785 - 1 ${ }^{7}$; same collection data as for holotype; Extraction1068, BOLD: AUGLY042-21; AM K.517930 - 1 ô; same collection data as for holotype; Extraction1087, BOLD: AUGLY044-21; AM K.517931

- 1 ô; same collection data as for holotype; Extraction1094, BOLD: AUGLY045-21; AM K.517932

- 1 ${ }^{2}$; same collection data as for holotype; Extraction1155, BOLD: AUGLY065-21; AM K.517933

- 1 (ethanol); same collection data as for holotype; Extraction1157, BOLD: AUGLY067-21; AM K.383786 • 1 O; Oxley Wild Rivers National Park, East Kunderang Track; -30.818056, 152.135; 7 Nov. 2015; D.M. Bray leg.; blue pan trap; Extraction1248, BOLD: AUGLY073-21; AM K.517937. - Queensland - 1 o ; Lamington National Park; -28.142, 153.133; 248 m a.s.1.; 8-18 Apr. 2007; C. Lambkin and N. Starick leg.; IBISCA Plot \# IQ-300-D rainforest Malaise trap; Extraction605, BOLD: AUMIC390-18; QM T208398 • 1 क; same collection data as for preceding; Extraction604, BOLD: AUMIC389-18; QM T208397 • 1 o ; same collection data as for preceding; Extraction193, BOLD: AUMIC080-18; QM T208393 - 1 q (ethanol); same collection data as for preceding; Extraction632, BOLD: AUMIC416-18; QM T208395 - 1 क (ethanol); same collection data as for preceding; 23 Sep.-5 Oct. 2014; Extraction623, BOLD: AUMIC409-18; QM T208394 • 1 o; same collection data as for preceding; 5-22 Oct. 2014; Extraction627, BOLD: AUMIC412-18; QM T208396.

\section{Description}

\section{Female}

Colouration. Gena without a pale spot; labrum mostly dark or reddish-brown; scape colour in ventral half uniformly paler than flagellomeres or paler than flagellomeres at proximal end; flagellomeres all black/dark brown; tegula normally dark (dark in holotype, sometimes paler in paratypes); wing veins uniformly black or brown, or with small lighter area proximally; anteromesoscutum all dark or dark with very slight orange patches on posterolateral corners; scutellar disk and metanotum dark; propodeum dark; fore coxa dark; mid coxa dark; hind coxa dark; fore femur pale yellow; mid femur pale yellow or light brown; hind femur dark reddish-brown, orange to light brown or dark; fore tibia pale yellow; mid tibia pale yellow or light brown; hind tibia darkening posteriorly; hind basitarsus light brown or dark reddish-brown; T1 dark; T2 sclerotised area dark; T2 lateral area same colour as sclerotised area, or only slightly paler or dark extends past indentation, but then pale; T3 dark, mostly dark with paler lateral areas or uniformly brown; T4+ dark or reddish-brown.

HolotyPe BODY MEASUREMENTS. Body length $2.4 \mathrm{~mm}$; fore wing length $2.3 \mathrm{~mm}$; antennal length slightly shorter than body length.

HEaD. Antennal flagellomere 14 length/width 1.25-2.00; antennal flagellomere 2 length/width 2.00 4.20; OOD/POD 1.57-2.50; IOD/POD 1.29-1.83.

Mesosoma. Anteromesoscutum sculpturing with shallow to deep punctures, space between punctures generally smaller than diameter of punctures; scutellar disk sculpturing with only very shallow punctures; 8-12 pits in scutellar sulcus; propodeum with median carina absent, shallow or strong punctures in anterior half, occasionally with small areas of shallow rugosity, posterior half of propodeum smooth or with shallow or strong rugose sculpturing, propodeum sometimes with smooth area in centre. 


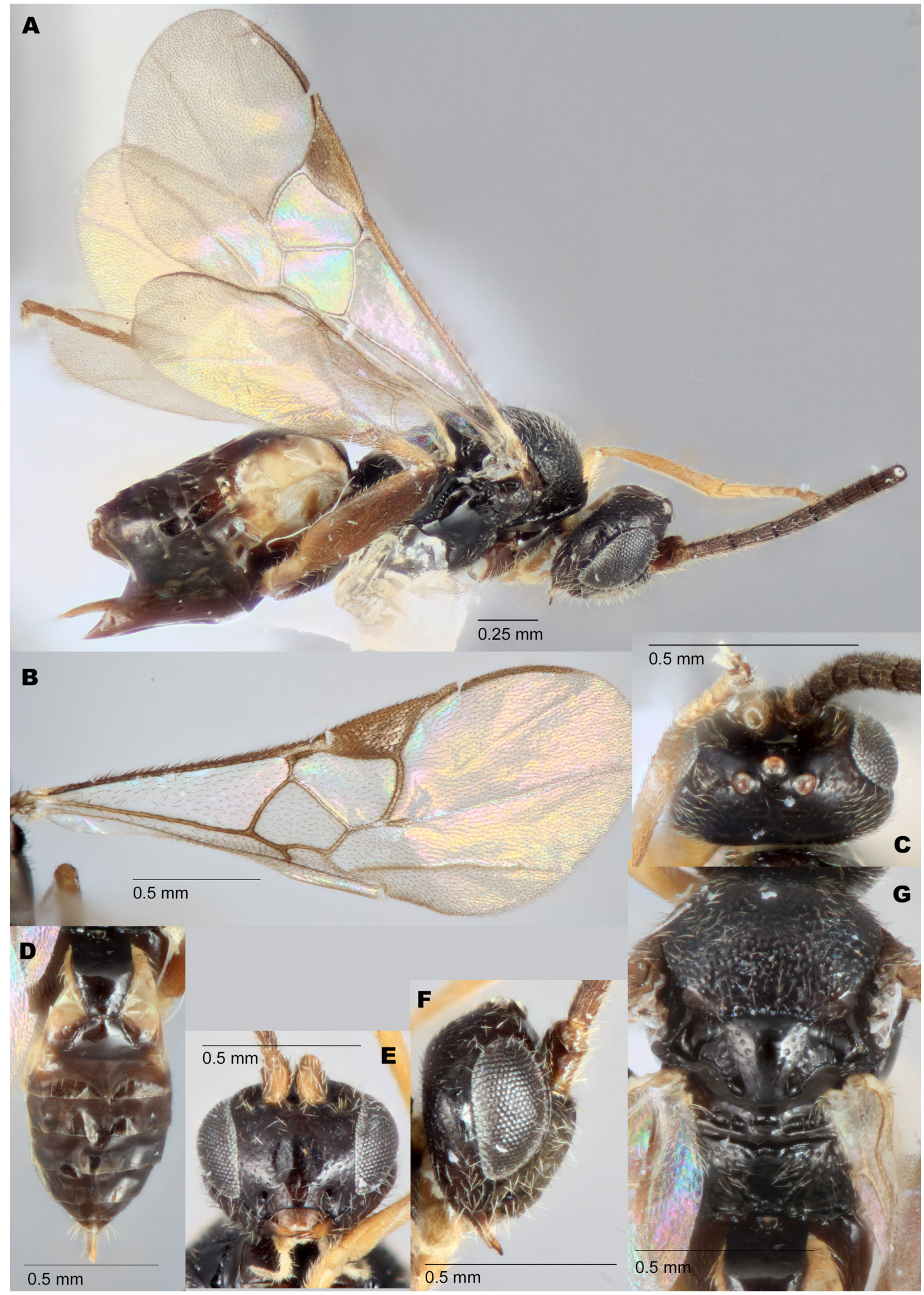

Fig. 29. Glyptapanteles doreyi Fagan-Jeffries, Bird \& Austin sp. nov., paratype, $q$ (ANIC 32 130330), 'clade B'. A. Lateral habitus. B. Fore wing. C. Dorsal head. D. Dorsal metasoma. E. Anterior head. F. Lateral head. G. Dorsal mesosoma. 
WINGS. Pterostigma length $0.53 \mathrm{~mm}$; pterostigma width $0.17 \mathrm{~mm} ; \mathrm{r} 0.15 \mathrm{~mm} ; 2 \mathrm{RS} 0.14 \mathrm{~mm} ; 2 \mathrm{~m} 0.09 \mathrm{~mm}$; $(\mathrm{RS}+\mathrm{M}) \mathrm{b} 0.08 \mathrm{~mm}$.

Metasoma. T1 lateral edges parallel for anterior $1 / 2$ to $2 / 3$ of length, then narrowing posteriorly; T1 sometimes shiny, smooth, or mostly smooth with some punctures in posterior half, or smooth in anterior half with indistinct sculpturing in posterior half; T1 length $0.34 \mathrm{~mm}$; T1 width at posterior edge $0.13 \mathrm{~mm}$; T2 an isosceles trapezoid, lateral edges straight; T2 smooth and shiny; T2 length $0.14 \mathrm{~mm}$; $\mathrm{T} 2$ width at posterior edge $0.31 \mathrm{~mm}$; ovipositor slightly protruding from end of metasoma.

\section{Male}

As female but with slightly longer antennae.

\section{Remarks}

Glyptapanteles doreyi sp. nov. has two wingless haplotypes (differing by a single base pair); however, as the COI divergence between specimens with the two different haplotypes is not consistent (i.e., one specimen has a $C O I$ sequence that is more closely related to specimens with the alternative wingless haplotype than to others) and the COI divergence among all specimens is not above $2 \%$, we consider all specimens to be one species. One of the wingless haplotypes is shared with the species G. arcanus sp. nov. and G. goodwinnoakes sp. nov. and both barcodes only differ by 1 bp from the species G. vergrandiacus sp. nov. and G. lessardi sp. nov. This group of species is very closely related and would benefit from future, more detailed taxonomic work to ensure that they are not simply divergent populations of the same species. For this species hypothesis, we have made the decision to split this large clade into different species based on the COI barcode sequences being $>3 \%$ divergent; however, we note that this is a hypothesis and is open to change with future work.

In the IQ-TREE analysis of the concatenated COI and wingless alignment, there are two clades within $G$. doreyi sp. nov. that differ, in their COI sequences, between $2.0 \%$ and $2.5 \%$. The first clade ('clade A') contains seven sequences (AUMIC416-18, AUMIC409-18, AUMIC080-18, AUGLY044-21, AUMIC412-18, AUMIC390-18, AUMIC389-18). Specimen AUGLY044-21 has the same wingless haplotype as the specimens in the second clade within G. doreyi sp. nov. ('clade B') whilst the other six specimens share a wingless haplotype that differs by one base pair from that of AUGLY044-21. We have made the conservative decision to combine these two clades into a single species due to the COI divergence being borderline of what is often used in Microgastrinae, lack of clear morphological differences and the wingless haplotype of AUGLY044-21 being identical to that of the alternate clade; however, we provide images of a representative of both clades and note that future evidence (particularly host data) may end up splitting this species further.

Glyptapanteles doreyi sp. nov. constitutes BIN BOLD:ABY0421 (clade B) and BOLD:AEC8663 (clade A). Using the BOLD Batch ID engine, the COI sequence of the holotype is $2.6 \%$ different from the most similar $C O I$ sequence from an Australian specimen (MCCAA7675-20; an undescribed lineage).

\section{Distribution}

This species is currently known from eastern NSW and from the ACT.

Glyptapanteles dowtoni Fagan-Jeffries, Bird \& Austin sp. nov. urn:1sid:zoobank.org:act:35AA0388-8874-46DB-8614-DF3CA15601B6

Figs $6 \mathrm{E}, 11 \mathrm{C}-\mathrm{D}, 30$

\section{Diagnosis}

Glyptapanteles dowtoni sp. nov. is in the G. mouldsi species group and can be separated from G. mouldsi sp. nov. by having S5+ dark and T4 6 with at least some dark areas, or completely dark. 
Glyptapanteles mouldsi sp. nov. has the metasomal sternites all pale yellow other than the hypopygium, which is dark and T4-5 all pale/yellow. With the limited specimens available, there was not a morphological character found that easily differentiates $G$. dowtoni sp. nov. from G. rixi sp. nov. The wingless sequences of the three species differ by at least $6 \mathrm{bp}$ and the COI differs by at least $4.3 \%$.

\section{Etymology}

This species is named for Professor Mark Dowton, who collected one of the paratypes, along with many other specimens, whilst kindly running a Malaise trap for this and other microgastrine projects at Mt Keira, NSW.

\section{Material examined}

\section{Holotype}

AUSTRALIA • $\bigcirc$; New South Wales, Royal National Park, near Waterfall Couranga Track near Hacking River; -34.1486, 151.0221; 40 m a.s.1.; 20 Jan.-6 Feb. 2020; K.M. Bayless and J.G. Lumbers leg.; 6 m Malaise trap over Waterfall Creek; Extraction1031, BOLD: AUGLY039-21; ANIC 32130361.

\section{Paratypes}

AUSTRALIA - New South Wales 1 क (ethanol); same collection data as for holotype; Extraction944, BOLD: AUGLY024-21; ANIC 32 130362 - 1 o (ethanol); same collection data as for holotype; Extraction1019, BOLD: AUGLY036-21; ANIC 32 130363 • 1 ( (ethanol); same collection data as for holotype; Extraction1121, BOLD: AUGLY054-21; ANIC 32 130364 • 1 o ; same collection data as for holotype; Extraction1126, BOLD: AUGLY057-21; ANIC 32 130365 • 1 ㅇ (ethanol); same collection data as for holotype; Extraction1270, BOLD: AUGLY074-21; ANIC 32 130366 • 1 ㅇ (ethanol); same collection data as for holotype; Extraction860, BOLD: AUGLY008-21; ANIC 32 130367 • 1 ठ (ethanol); same collection data as for holotype; Extraction935, BOLD: AUGLY021-21; ANIC 32130368 • 1 ¿ ; same collection data as for holotype; Extraction946, BOLD: AUGLY025-21; ANIC 32130369 - 1 क; Mt Keira; -34.416, 150.882; 5-20 Mar. 2020; M. Dowton leg.; Malaise trap, EFJ2020MT29; Extraction878, BOLD: AUGLY014-21; AM K.517934. - Queensland • 1 क; Lamington National Park; -28.155, 153.139; 282 m a.s.1.; 13-23 Jan. 2007; C. Lambkin and N. Starick leg.; IBISCA Plot \# IQ-300-B rainforest Malaise trap, bulk vial 22139; Extraction1465, BOLD: AUGLY083-21; QM T250948 • 1 o (ethanol); same collection data as for preceding; Extraction1466, BOLD:AUGLY084-21; QM T250949• 1 q; same collection data as for preceding; Extraction1468, BOLD: AUGLY086-21; QM QM T250950 • 1 ; ; same collection data as for preceding; Extraction1469, BOLD: AUGLY087-21; QM T250951 • 1 (ethanol); same collection data as for preceding; Extraction1470, BOLD: AUGLY088-21; QM T250952 - 1 9; same collection data as for preceding; Extraction600, BOLD: AUMIC386-18; QM T208403 • 1 q; same collection data as for preceding; Extraction602, BOLD: AUMIC388-18; QM T208404 • 1 9 ; Cape Tribulation, Cow Bay, Daintree Discovery Centre; -16.2382, 145.427; 86 m a.s.1.; 22 Aug. 2015; C.J. Bennett leg.; BIOUG44012-D01, BOLD: GMQQY084-18; QM T250953.

\section{Description}

\section{Female}

Colouration. Gena without a pale spot; labrum reddish-brown; scape colour in ventral half uniformly paler than flagellomeres; flagellomeres darkening distally; tegula dark or pale; wing veins uniformly black or brown, or with small lighter area proximally; anteromesoscutum all dark or dark with very slight orange patches on posterolateral corners; scutellar disk and metanotum dark; propodeum dark; fore coxa dark; mid coxa dark; hind coxa dark; fore femur pale yellow; mid femur pale yellow; hind femur pale yellow; fore tibia pale yellow; mid tibia pale yellow; hind tibia darkening posteriorly; hind basitarsus light brown; T1 dark; T2 sclerotised area dark reddish-brown, pale or orange to light brown; T2 lateral area same colour as sclerotised area, or only slightly paler or dark extends past indentation, but then pale; T3 mostly pale with darker patch in centre, uniformly brown or pale; T4+ reddish-brown, or T4 pale or pale with dark patch in centre; T5-7 darkening posteriorly. 


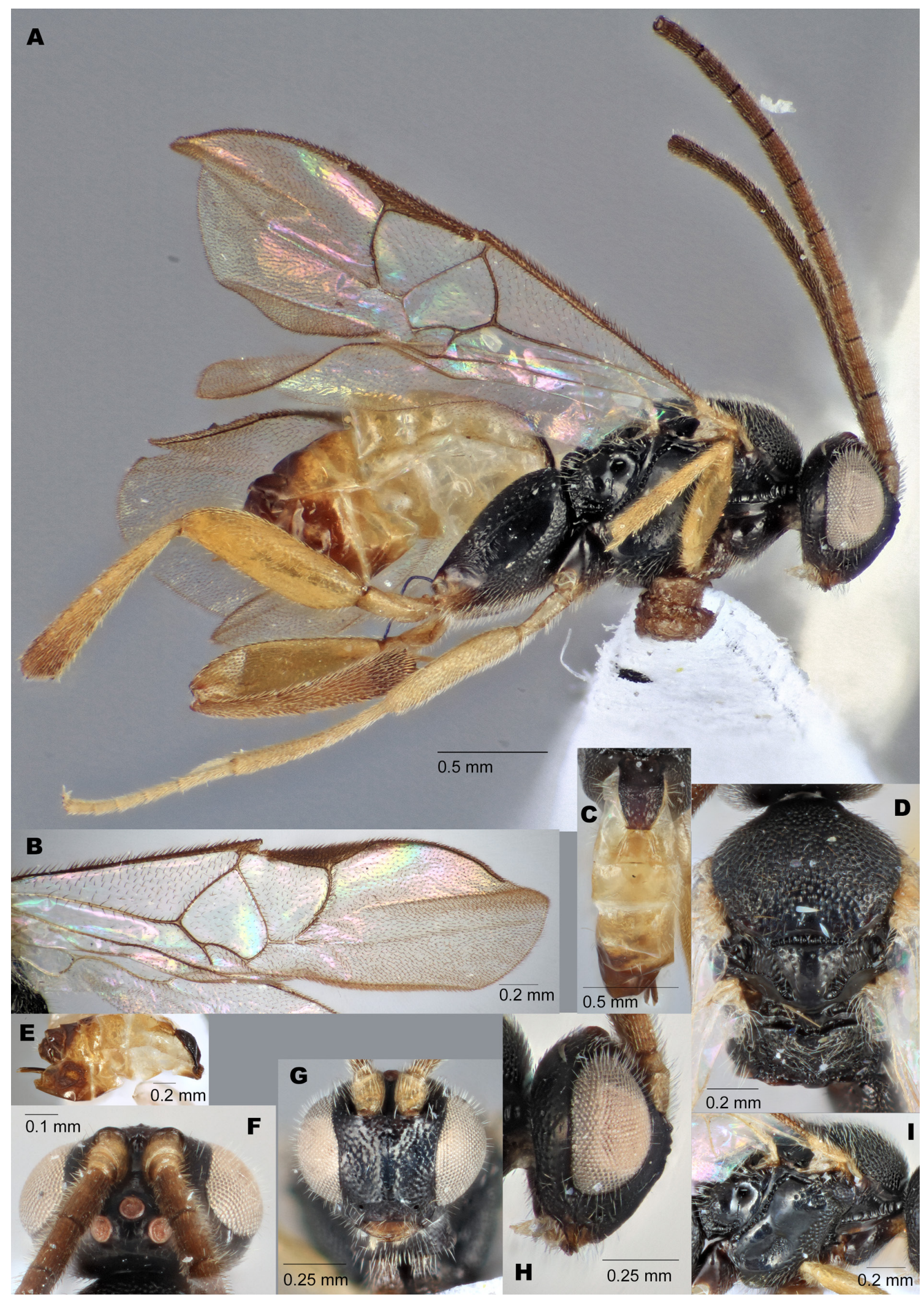

Fig. 30. Glyptapanteles dowtoni Fagan-Jeffries, Bird \& Austin sp. nov., paratype, $q$ (QM T250953). A. Lateral habitus. B. Fore wing. C. Dorsal metasoma. D. Dorsal mesosoma. E. Lateral metasoma. F. Dorsal head. G. Anterior head. H. Lateral head. I. Lateral mesosoma. 
Holotype Body MEASUREMENTs. Body length $2.3 \mathrm{~mm}$; fore wing length $2.3 \mathrm{~mm}$; antennal length slightly longer than body length.

HeAD. Antennal flagellomere 14 length/width 2.00-2.16; antennal flagellomere 2 length/width $2.55-$ 3.83; OOD/POD 1.33-2.17; IOD/POD 1.11-1.67.

Mesosoma. Anteromesoscutum sculpturing with shallow to deep punctures, space between punctures a mixture of smaller than diameter of punctures and of similar size, often smoother in posterior centre; scutellar disk sculpturing with deep, sparse, irregularly spaced punctures, more common on anterior lateral edges, or with sparse punctures scattered over most of area; 9-12 pits in scutellar sulcus; propodeum with median carina absent, propodeum strongly sculptured, punctate reticulate to rugose, sometimes with smooth shining areas in posterior third.

WINGS. Pterostigma length $0.55 \mathrm{~mm}$; pterostigma width $0.17 \mathrm{~mm} ; \mathrm{r} 0.18 \mathrm{~mm} ; 2 \mathrm{RS} 0.15 \mathrm{~mm} ; 2 \mathrm{~m} 0.07 \mathrm{~mm}$; $(\mathrm{RS}+\mathrm{M}) \mathrm{b} 0.11 \mathrm{~mm}$.

Metasoma. T1 lateral edges parallel for anterior $1 / 2$ to $2 / 3$ of length, then narrowing posteriorly or with lateral edges parallel for anterior $3 / 4$ of length, then narrowing posteriorly; T1 smooth with shallow sculpturing along lateral edges or smooth and shiny, some shallow scattered punctures on lateral edges and posterior half; T1 length $0.38 \mathrm{~mm}$; T1 width at posterior edge $0.11 \mathrm{~mm}$; T2 an isosceles trapezoid, lateral edges straight or with curved lateral and anterior edges, becoming arch- or semicircle-shaped; T2 smooth, sometimes shiny, some indistinct sculpturing on postero-lateral corners; T2 length $0.14 \mathrm{~mm} ; \mathrm{T} 2$ width at posterior edge $0.19 \mathrm{~mm}$; ovipositor slightly protruding from end of metasoma.

\section{Male}

As female.

\section{Remarks}

Glyptapanteles dowtoni sp. nov. constitutes BIN: BOLD:ADL2613 and is 1.61\% (p-dist.) divergent from the closest BIN in the database (BOLD:AAH1085). BOLD:AAH1085 has two sequences within it (one of which is public: ASPNI556-09 from Papua New Guinea). This sequence was not included in the original analysis as it is not identified to genus level in BOLD. With the low p-dist. between these two BINs, we consider it possible that they may be the same species, which would mean that G. dowtoni sp. nov. is also present in Papua New Guinea. Morphological examination should be completed and a second gene should be sequenced to confirm.

Using the BOLD Batch ID engine, the COI barcode of the holotype is $2.8 \%$ different from the most similar COI sequence from an Australian specimen (AUGLY141-21; an undescribed lineage, with a single specimen). Fourteen specimens were able to be sequenced for the wingless gene and share a unique barcode that differs by a minimum of $3 \mathrm{bp}$ from all other species with available sequence data.

\section{Distribution}

This species is currently known from southern QLD and eastern NSW.

Glyptapanteles drioplanetus Fagan-Jeffries \& Austin, 2021 Figs 15A, 31

\section{Diagnosis}

Glyptapanteles drioplanetus can be separated from the other described species of Glyptapanteles in Australia by the combination of the following characters: gena without a pale spot, T1 and T2 dark, 


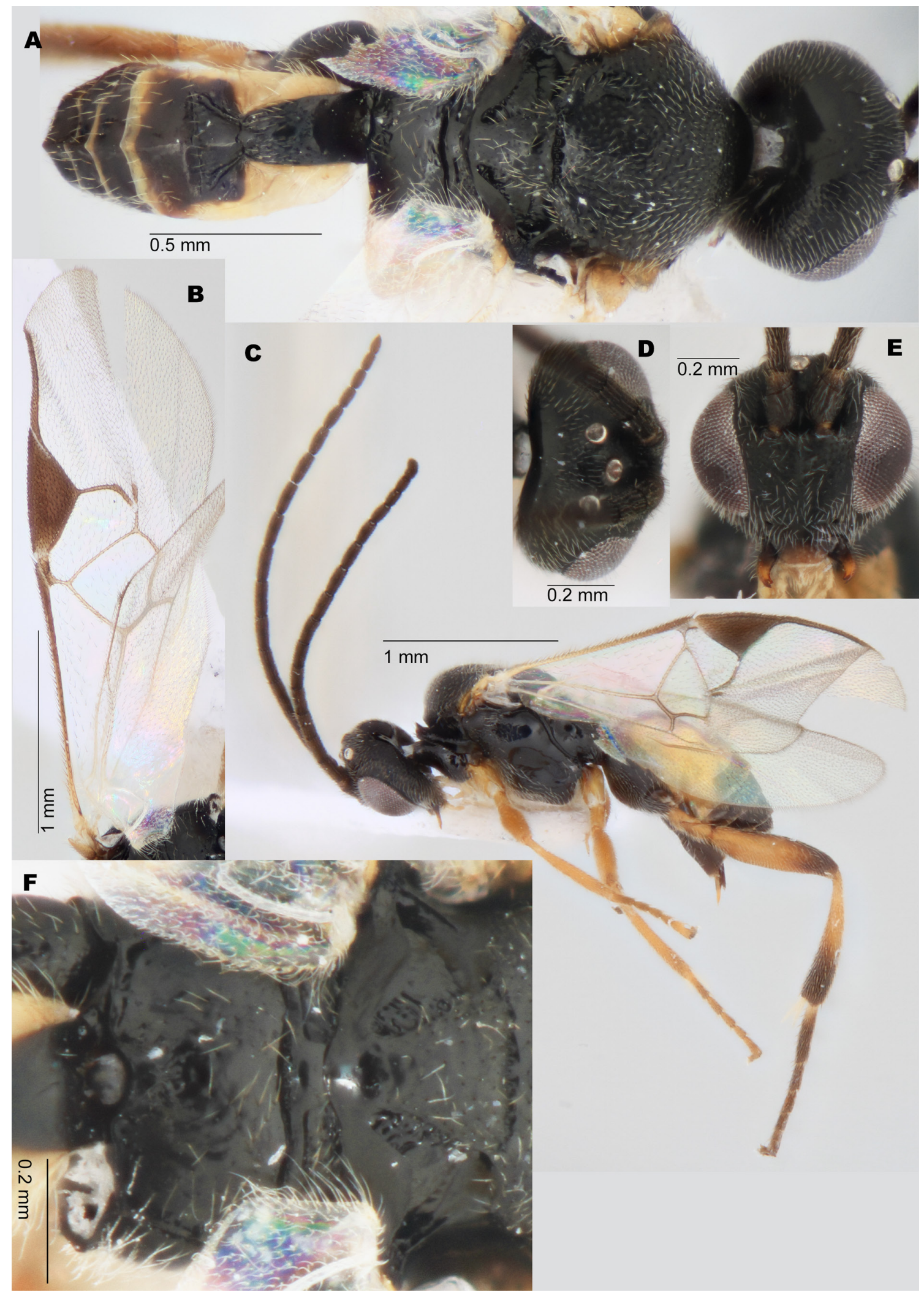

Fig. 31. Glyptapanteles drioplanetus Fagan-Jeffries \& Austin, 2021, holotype, $q$ (WAM WAME10965). A. Dorsal habitus. B. Fore wing. C. Lateral habitus. D. Dorsal head. E. Anterior head. F. Scutellar disk and propodeum. Images from Fagan-Jeffries \& Austin (2021: fig. 5). 
anteromesoscutum very smooth, with only shallow and regular punctures, no orange markings on the postero-lateral anteromesoscutum, propodeum very smooth with only shallow punctures and the median carina entirely absent, T1 narrowing slightly for most of length, distal ventral half of antennal scape darker than flagellomeres, hind femur pale anteriorly, darkening posteriorly, tegula pale, labrum pale.

\section{Material examined}

\section{Holotype}

AUSTRALIA - ; South Australia, Macclesfield Primary School; -35.17084, 138.84025; 14 Oct.-6 Nov. 2019; E. Fagan-Jeffries and Macclesfield PS yr. 5-7 class leg.; Malaise Trap EFJ2020MT9; Extraction1033, BOLD: AUMIC549-20; SAMA 32-45153.

\section{Paratypes}

AUSTRALIA - South Australia • 1 q; Douglas Scrub; -35.1844, 138.6; 27-28 Sep. 2010; G.S. Taylor and S. Mantel leg.; Malaise Trap 2010 001; Extraction79, BOLD: AUMIC507-18; SAMA 32-45154 - 1 o; Gleneagle State Forest; -32.2711, 116.163; 29 Nov. 2005; M.S. Harvey leg.; Malaise trap; Extraction117, BOLD: AUMIC019-18; WAM WAME10965.

\section{Remarks}

This species was recently described and we therefore only provide a diagnosis here. Glyptapanteles drioplanetus constitutes BIN: BOLD:ADL3660 and is 2.56\% (p-dist.) from the nearest neighbour (BOLD:AEI2300; an undescribed lineage from Australia, with two specimens).

Glyptapanteles eburneus Fagan-Jeffries, Bird \& Austin sp. nov. urn:lsid:zoobank.org:act:814EBC38-DD57-4503-87B3-01C47A2A517D

Figs $6 \mathrm{~B}, 10 \mathrm{~A}, 32$

\section{Diagnosis}

Glyptapanteles eburneus sp. nov. is in the G. eburneus species group and can be separated from G. foraminous sp. nov. by having T2 pale, the same colour as T1 (G. foraminous sp. nov. has T2 dark). Additionally, the wingless sequences of the two holotype specimens are distinct and differ by $5 \mathrm{bp}$.

\section{Etymology}

The species epithet 'eburneus' is a Latin adjective for 'ivory' and refers to the pale colouration of T1-2.

\section{Material examined}

\section{Holotype}

AUSTRALIA - ; New South Wales, Royal National Park. south end, Lady Carrington Drive Rainforest; -34.1482, 151.031; 24 Nov. 1996; A.D. Austin leg.; Extraction439, BOLD: AUMIC274-18; AM K.517935.

\section{Description}

\section{Female}

Colouration. Gena without a pale spot; labrum pale; scape colour in ventral half uniformly paler than flagellomeres; flagellomeres darkening distally; tegula pale; wing veins uniformly black or brown, or with small lighter area proximally; anteromesoscutum dark with significant orange patches on posterolateral corners; scutellar disk and metanotum dark; propodeum dark; fore coxa pale yellow; mid coxa pale yellow; hind coxa pale yellow; fore femur pale yellow; mid femur pale yellow; hind femur pale yellow; fore tibia pale yellow; mid tibia pale yellow; hind tibia darkening posteriorly; hind basitarsus light 


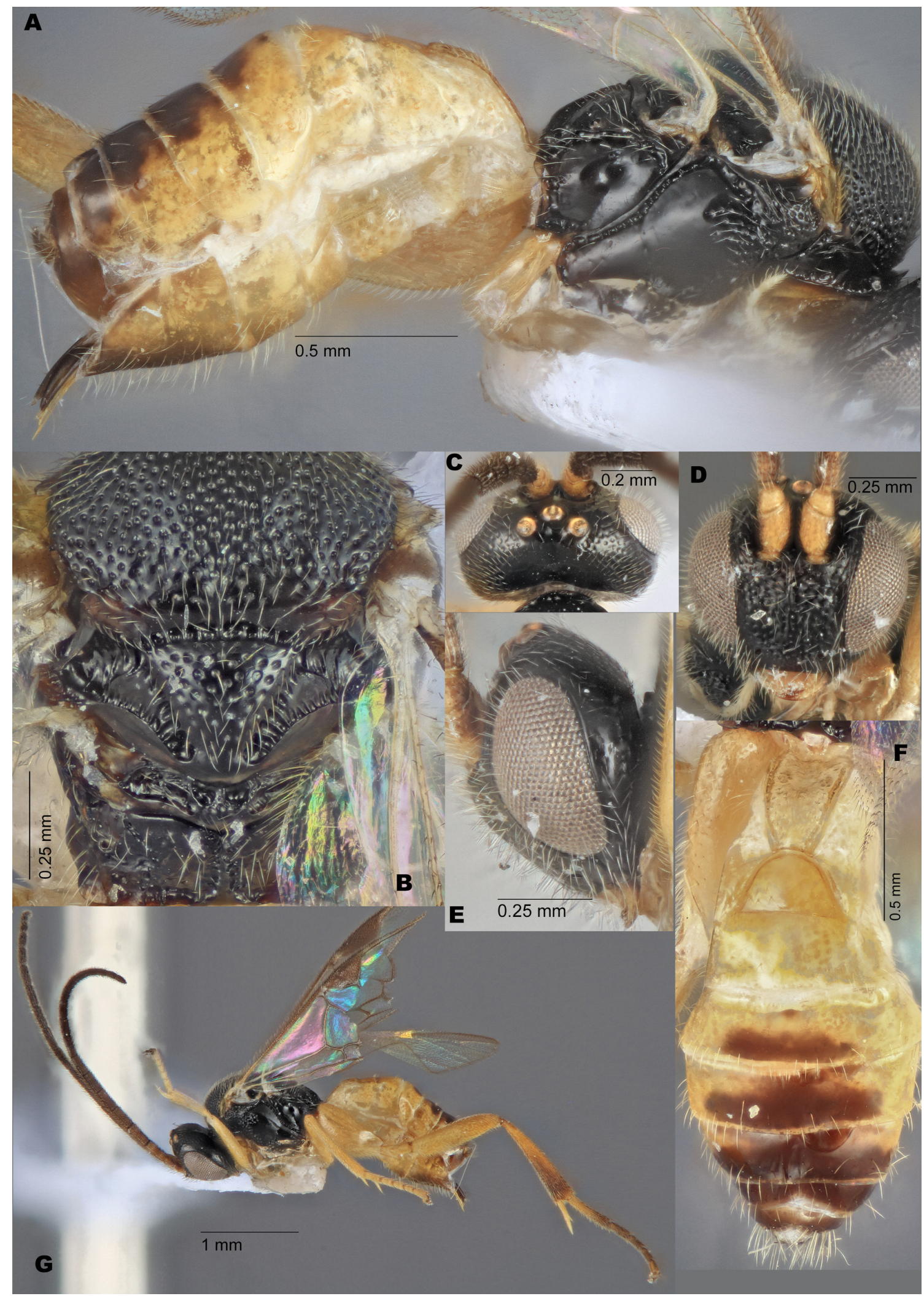

Fig. 32. Glyptapanteles eburneus Fagan-Jeffries, Bird \& Austin sp. nov., holotype, o (AM K.517935). A. Lateral body. B. Dorsal mesosoma. C. Dorsal head. D. Anterior head. E. Lateral head. F. Dorsal metasoma. G. Lateral habitus. 
brown; T1 pale; T2 sclerotised area pale; T2 lateral area same colour as sclerotised area, or only slightly paler; T3 pale; T4 pale with dark patch in centre, then darkening posteriorly on T5-7.

Holotype Body MEASUREMENTS. Body length $2.7 \mathrm{~mm}$; fore wing length $2.7 \mathrm{~mm}$; antennal length slightly longer than body length.

HEAD. Face densely sculptured, punctate reticulate; antennal flagellomere 14 length/width 2.16; antennal flagellomere 2 length/width 3.62; OOD/POD 1.75; IOD/POD 1.75.

Mesosoma. Anteromesoscutum sculpturing with deep punctures, space between punctures a mixture of smaller than diameter of punctures and of similar size; scutellar disk sculpturing with deep irregularly spaced punctures; nine pits in scutellar sulcus; propodeum with median carina present and complete, rest of propodeum mostly smooth.

WINGS. Pterostigma length $0.62 \mathrm{~mm}$; pterostigma width $0.23 \mathrm{~mm} ; \mathrm{r} 0.18 \mathrm{~mm} ; 2 \mathrm{RS} 0.16 \mathrm{~mm} ; 2 \mathrm{~m} 0.10 \mathrm{~mm}$; $(\mathrm{RS}+\mathrm{M}) \mathrm{b} 0.10 \mathrm{~mm}$.

METASOMA. T1 wedge-shaped, narrowing posteriorly for entirety of length, lateral edges straight (but not parallel); T1 mostly smooth, some punctures in posterior half; T1 length $0.4 \mathrm{~mm}$; T1 width at posterior edge $0.12 \mathrm{~mm}$; T2 with curved lateral and anterior edges, becoming arch- or semicircle-shaped; T2 smooth and shiny; T2 length $0.17 \mathrm{~mm}$; T2 width at posterior edge $0.3 \mathrm{~mm}$; ovipositor slightly protruding from end of metasoma.

\section{Male \\ Unknown.}

\section{Remarks}

Glyptapanteles eburneus sp. nov. constitutes BIN: BOLD:ADL5650 and is 2.4\% (p-dist.) divergent from the closet BIN in the database (BOLD:ADL3303; Glyptapanteles foraminous sp. nov.).

Using the BOLD Batch ID engine, the COI barcode of the holotype is $2.3 \%$ different from the most similar COI sequence from an Australian specimen (AUMIC069-18; Glyptapanteles foraminous sp. nov.). The type specimen was able to be sequenced for the wingless gene, which differs by a minimum of $5 \mathrm{bp}$ from all other species with available sequence data.

\section{Distribution}

This species is currently represented by a single specimen from Royal National Park, just south of Sydney.

Glyptapanteles erucadesolator Fagan-Jeffries, Bird \& Austin sp. nov. urn:1sid:zoobank.org:act:22704EB8-67CA-41FF-BACE-0B9CD656788C

Fig. 33

\section{Diagnosis}

Glyptapanteles erucadesolator sp. nov. is in the G. arcanus species group and can be separated from other members of the species group, other than from G. lambkinae sp. nov., by having the propodeum with very coarse and strong rugose sculpting and $\mathrm{T} 1$ with strong sculpturing. Glyptapanteles arcanus sp. nov., G. vergrandiacus sp. nov. and G. goodwinnoakes sp. nov. also have coarse sculpturing on the propodeum (although to a lesser degree), but T1 is either smooth or with clear punctures (but not rugose sculpturing). It is noted, however, that all these species are morphologically very similar and 
identifications should be made with DNA barcodes. There was not a morphological character found to easily differentiate Glyptapanteles erucadesolator sp. nov. from G. lambkinae sp. nov., which are sister lineages in the current phylogeny (Fig. 2); however, as the species differ by $2 \mathrm{bp}$ in the wingless sequence and there is $>6 \%$ divergence in the COI barcode, we feel confident that these are different species.

\section{Etymology}

The species epithet 'erucadesolator' is derived from the Latin words 'eruca' ('caterpillar') and 'desolator' ('destroyer') and refers to the lepidopteran parasitoid habit of this subfamily. It is a noun in apposition.

\section{Material examined}

\section{Holotype}

AUSTRALIA • O; Queensland, Hermit Park; -19.2828, 146.801; 10 m a.s.1.; 7 Jul. 2008; G. Cocks leg.; Malaise trap; gvc9348-1L, BOLD: HYQT050-08; QM T250954.

\section{Paratypes}

AUSTRALIA - Northern Territory • 1 q; Gregory National Park, Station Creek, $0.2 \mathrm{~km}$ NNW of Bullita Camp Ground; -16.1117, 130.423; 12 Jan. 2001; M.E. Irwin, F.D. Parker and C. Lambkin leg.; Extraction160, BOLD: AUMIC054-18; ANIC 32 130199. - Queensland • 1 đ ; Hervey Range; -19.3812, 146.449; 380 m a.s.1.; 22 Aug. 2017; G. Cocks leg.; Malaise trap; gvcT10741, BOLD: GCQT2624-18; QM T250955.

\section{Description}

\section{Female}

Colouration. Gena without a pale spot; labrum mostly dark; scape colour in ventral half uniformly paler than flagellomeres or the same colour or darker than flagellomeres; flagellomeres darkening distally; tegula pale; wing veins uniformly black or brown, or with small lighter area proximally; anteromesoscutum all dark or dark with very slight orange patches on posterolateral corners; scutellar disk and metanotum dark; propodeum dark; fore coxa dark; mid coxa dark; hind coxa dark; fore femur orange to light brown; mid femur orange to light brown; hind femur orange to light brown; fore tibia orange to light brown; mid tibia orange to light brown; hind tibia darkening posteriorly; hind basitarsus light brown; T1 dark; T2 sclerotised area dark or dark reddish-brown; T2 lateral area dark extends past indentation, but then pale; T3 mostly dark with paler lateral areas or uniformly brown; T4+ dark or reddish-brown.

Holotype Body MEASUREMENTs. Body length $2.4 \mathrm{~mm}$; fore wing length $2.5 \mathrm{~mm}$.

HEAD. Antennal flagellomere 14 length/width 2.66; antennal flagellomere 2 length/width 2.85; OOD/ POD 2.50-2.57; IOD/POD 1.57-2.17.

Mesosoma. Anteromesoscutum sculpturing with densely scattered deep and shallow punctures of irregular size; scutellar disk sculpturing with deep irregularly spaced punctures; 8-12 pits in scutellar sulcus; propodeum with median carina absent, propodeum strongly sculptured, punctate reticulate to rugose, with smooth shining areas in posterior third.

Wings. Pterostigma length $0.54 \mathrm{~mm}$; pterostigma width $0.2 \mathrm{~mm} ; \mathrm{r} 0.17 \mathrm{~mm} ; 2 \mathrm{RS} 0.16 \mathrm{~mm} ; 2 \mathrm{~m} 0.09 \mathrm{~mm}$; $(\mathrm{RS}+\mathrm{M}) \mathrm{b} 0.12 \mathrm{~mm}$.

Metasoma. T1 lateral edges parallel for anterior $1 / 2$ to $2 / 3$ of length, then narrowing posteriorly, or lateral edges parallel for anterior $3 / 4$ of length, then narrowing posteriorly; $\mathrm{T} 1$ mostly smooth, some shallow rugose 




Fig. 33. Glyptapanteles erucadesolator Fagan-Jeffries, Bird \& Austin sp. nov. A, C, F. Paratype, $q$ (ANIC 32 130199). B, D-E, G. Holotype, $q$ (QM T250954). A. Lateral habitus. B. Dorsal mesosoma. C. Dorsal head. D. Fore wing. E. Dorsal propodeum and metasoma. F. Anterior head. G. Ovipositor sheaths. 
sculpturing in posterior half; T1 length $0.37 \mathrm{~mm}$; T1 width at posterior edge $0.08 \mathrm{~mm}$; T2 an isosceles trapezoid, lateral edges straight; T2 smooth and shiny, some shallow punctures along posterior edge; T2 length $0.16 \mathrm{~mm}$; T2 width at posterior edge $0.27 \mathrm{~mm}$; ovipositor slightly protruding from end of metasoma.

\section{Male}

As female, antennal flagellomeres slightly longer.

\section{Remarks}

Glyptapanteles erucadesolator sp. nov. constitutes BIN BOLD:AAG2095 and is 5.13\% (p-dist.) divergent from the closet BIN in the database (BOLD:AEI7392; an undescribed lineage, from Australia).

Using the BOLD Batch ID engine, the COI barcode of the holotype is $5.9 \%$ different from the most similar COI sequence from an Australian specimen (AUGLY160-21; an undescribed lineage, with one specimen). One of the paratype specimens (AUMIC054-18) was sequenced for the wingless gene, which differs by a minimum of $2 \mathrm{bp}$ from all other species with available sequence data.

\section{Distribution}

This species is currently known from northern Australia: from Gregory National Park in the NT and northern QLD.

Glyptapanteles ferrugineus Fagan-Jeffries, Bird \& Austin sp. nov. urn:Isid:zoobank.org:act:FF8A3C0E-096B-40AE-ADA3-33E4069EF15E

Figs 9A, 34

\section{Diagnosis}

Glyptapanteles ferrugineus sp. nov. is in the G. albigena species group and can be separated from the other members of the species group by the pale spot on the gena being small but clearly visible, $\mathrm{T} 2$ dark, propodeal median carina completely absent, hind femur light brown to pale and mesosoma with a strong dark red tinge.

\section{Etymology}

The species epithet, 'ferrugineus', is a Latin adjective for 'rust-coloured' and refers to the red tinge for the mesosoma.

\section{Material examined}

\section{Holotype}

AUSTRALIA • + ; Western Australia, 23 km NNW ofAlbany; -34.8532, 117.809; 16 Nov. 2000; T. Simmul and S. Cunningham leg.; Malaise in remnant vegetation; Extraction144, BOLD: AUMIC042-18; ANIC 32130189.

\section{Description}

\section{Female}

Colouration. Gena with a pale spot; labrum reddish-brown; scape colour in ventral half uniformly paler than flagellomeres; flagellomeres uniformly reddish-brown; tegula pale; wing veins uniformly pale; anteromesoscutum dark with red tinge; scutellar disk and metanotum dark with red tinge; propodeum dark with red tinge; fore coxa pale yellow; mid coxa pale yellow; fore femur pale yellow; mid femur pale yellow; hind femur pale yellow; fore tibia pale yellow; mid tibia pale yellow; hind basitarsus light brown; T1 dark reddish-brown; T2 sclerotised area dark reddish-brown; T2 lateral area dark, extends past indentation, but then pale; T3 mostly dark with paler lateral areas; T4+ reddish-brown. 


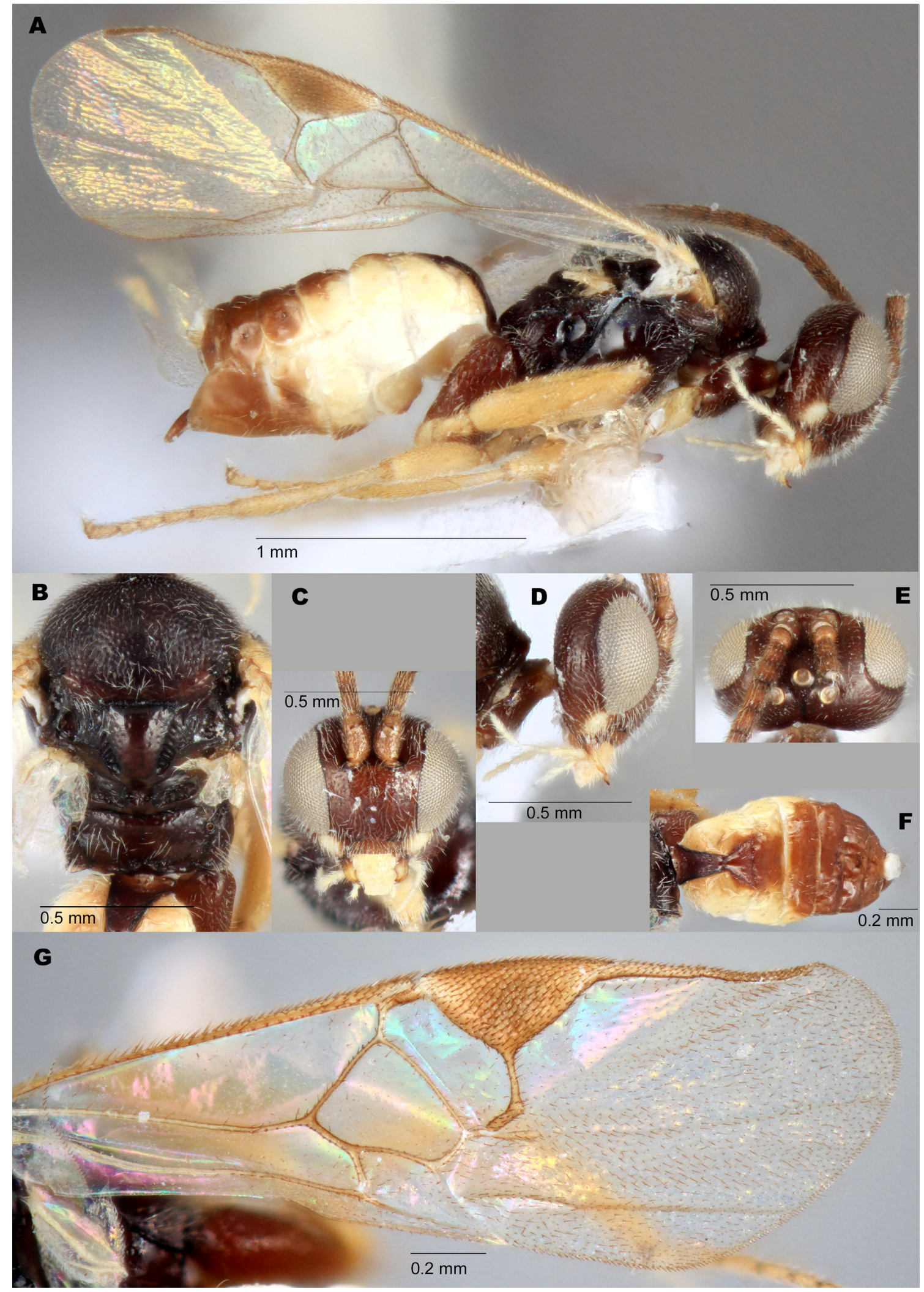

Fig. 34. Glyptapanteles ferrugineus Fagan-Jeffries, Bird \& Austin sp. nov., holotype, $ᄋ$ (ANIC: 32 130189). A. Lateral habitus. B. Dorsal mesosoma. C. Anterior head. D. Lateral head. E. Dorsal head. F. Dorsal metasoma. G. Fore wing. 
Holotype Body MEASUREMENTs. Body length $2.2 \mathrm{~mm}$; fore wing length $2.1 \mathrm{~mm}$; antennal length slightly shorter than body length.

HEAD. Antennal flagellomere 14 length/width 1.66; antennal flagellomere 2 length/width 3.33; OOD/ POD 1.71; IOD/POD 1.57.

Mesosoma. Anteromesoscutum sculpturing with shallow punctures, space between punctures generally smaller than diameter of punctures; scutellar disk sculpturing with only very shallow punctures; eight pits in scutellar sulcus; propodeum with median carina absent, very smooth and shiny, only very shallow punctures associated with setae.

Wings. Pterostigma length $0.48 \mathrm{~mm}$; pterostigma width $0.22 \mathrm{~mm} ; \mathrm{r} 0.12 \mathrm{~mm} ; 2 \mathrm{RS} 0.1 \mathrm{~mm} ; 2 \mathrm{~m} 0.13 \mathrm{~mm}$; $(\mathrm{RS}+\mathrm{M}) \mathrm{b} 0.07 \mathrm{~mm}$.

Metasoma. T1 wedge-shaped, narrowing posteriorly for entirety of length, lateral edges straight (but not parallel); T1 smooth and shiny; T1 length $0.31 \mathrm{~mm}$; T1 width at posterior edge $0.07 \mathrm{~mm}$; T2 an isosceles trapezoid, lateral edges straight; T2 smooth and shiny; T2 length $0.13 \mathrm{~mm}$; T2 width at posterior edge $0.21 \mathrm{~mm}$; ovipositor slightly protruding from end of metasoma.

\section{Male}

Unknown.

\section{Remarks}

Glyptapanteles ferrugineus sp. nov. constitutes BIN: BOLD:ADL4089 and is 4.17\% (p-dist.) divergent from the closet BIN in the database (BOLD:ABA6213; Glyptapanteles austrinus sp. nov.).

Using the BOLD Batch ID engine, the COI barcode of the holotype is $4.0 \%$ different from the most similar COI sequence from an Australian specimen (HYAT371-11; Glyptapanteles austrinus sp. nov.). The holotype was able to be sequenced for the wingless gene, which differs by a minimum of 5 bp from all other species with available sequence data.

\section{Distribution}

This species is known only from Albany in southern WA.

Glyptapanteles foraminous Fagan-Jeffries, Bird \& Austin sp. nov. urn:lsid:zoobank.org:act:F8AD515F-46A6-4AA9-996B-D05FB4650188

Fig. 35

\section{Diagnosis}

Glyptapanteles foraminous sp. nov. is in the G. eburneus species group and can be separated from G. eburneus sp. nov. by having T2 dark; G. eburneus sp. nov. has T2 pale. Additionally, the wingless sequences of the two holotype specimens are distinct and differ by $5 \mathrm{bp}$.

\section{Etymology}

The species epithet 'foraminous' is a Latin adjective meaning 'full of holes' and refers to the highly sculptured mesosoma with dense indentations.

\section{Material examined}

\section{Holotype}

AUSTRALIA • +; Queensland, Cainbable Quarry, "OF”; -28.145, 153.113; 3-19 Feb. 2009; F. Turco leg.; Extraction182, BOLD: AUMIC069-18; QM T208408. 


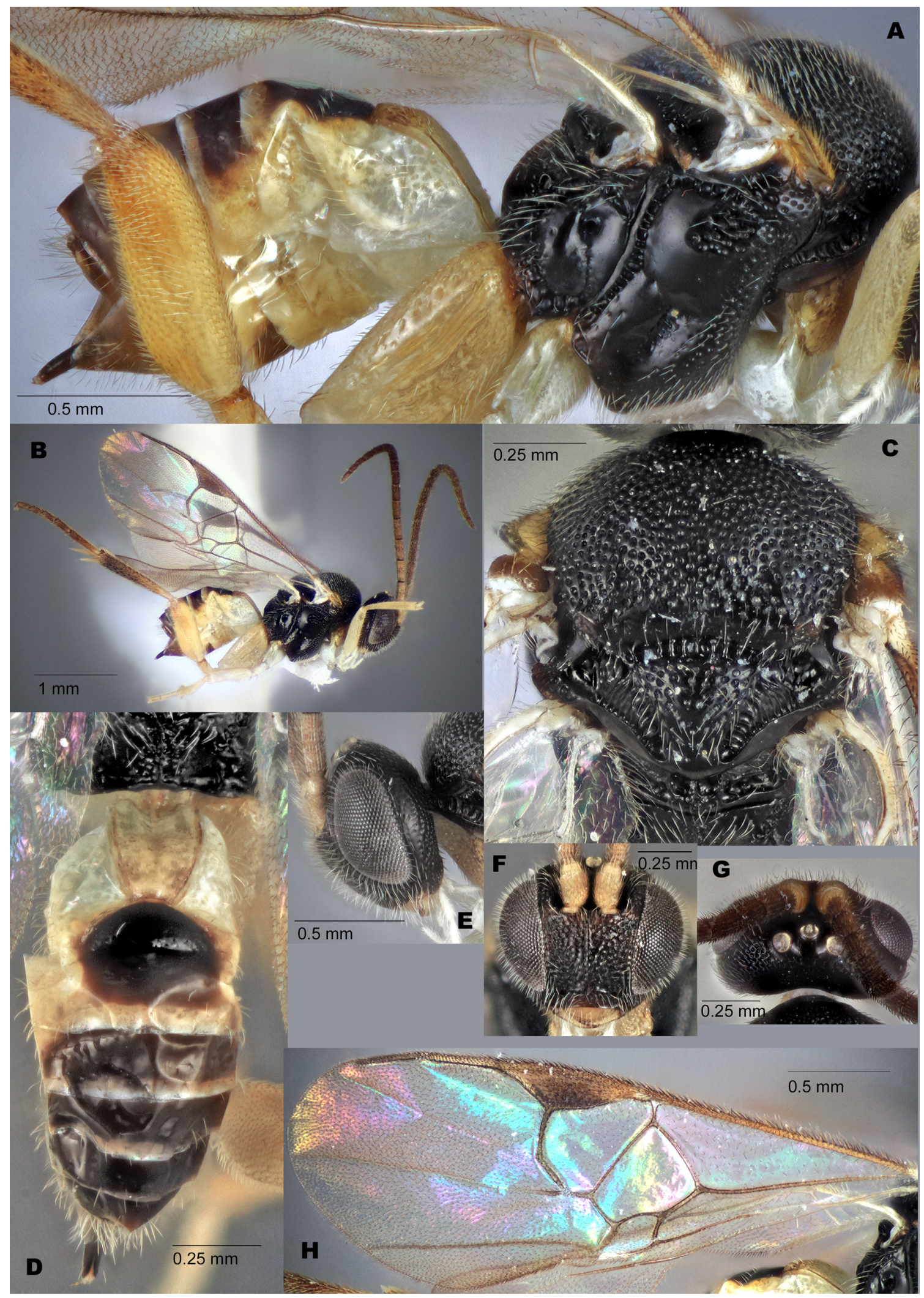

Fig. 35. Glyptapanteles foraminous Fagan-Jeffries, Bird \& Austin sp. nov., holotype, + (QM T208408). A. Lateral mesosoma. B. Lateral habitus. C. Dorsal mesosoma. D. Dorsal metasoma. E. Lateral head. F. Anterior head. G. Dorsal head. H. Fore wing. 


\section{Description}

\section{Female}

Colouration. Gena without a pale spot; labrum pale; scape colour in ventral half uniformly paler than flagellomeres; flagellomeres uniformly reddish-brown; tegula pale; wing veins uniformly black or brown, or with small lighter area proximally; anteromesoscutum dark with very slight orange patches on posterolateral corners; scutellar disk and metanotum dark; propodeum dark; fore coxa white; mid coxa white; hind coxa pale yellow; mid femur pale yellow; hind femur pale yellow; mid tibia pale yellow; hind tibia darkening posteriorly; hind basitarsus light brown; T1 pale; T2 sclerotised area dark; T2 lateral area much paler; T3 mostly dark with paler lateral areas; T4+ reddish-brown.

Holotype Body MEASUREMENTs. Body length $2.0 \mathrm{~mm}$; fore wing length $2.0 \mathrm{~mm}$; antennal length slightly longer than body length.

HEAD. Face densely sculptured, punctate reticulate; antennal flagellomere 14 length/width 2.71; antennal flagellomere 2 length/width 3.57; OOD/POD 1.75; IOD/POD 1.88.

Mesosoma. Anteromesoscutum sculpturing with deep punctures, space between punctures a mixture of smaller than diameter of punctures and of similar size; scutellar disk sculpturing with deep irregularly spaced punctures; 10 pits in scutellar sulcus; propodeum with median carina present and complete, rest of propodeum mostly smooth.

WINGs. Pterostigma length $0.62 \mathrm{~mm}$; pterostigma width $0.21 \mathrm{~mm}$; $\mathrm{r} 0.22 \mathrm{~mm}$; $2 \mathrm{RS} 0.19 \mathrm{~mm} ; 2 \mathrm{~m} 0.09 \mathrm{~mm}$; $(\mathrm{RS}+\mathrm{M}) \mathrm{b} 0.11 \mathrm{~mm}$.

Metasoma. T1 lateral edges parallel for anterior $1 / 2$ to $2 / 3$ of length, then narrowing posteriorly; T1 mostly smooth, some punctures in posterior half; T1 length $0.5 \mathrm{~mm}$; T1 width at posterior edge $0.1 \mathrm{~mm}$; T2 with curved lateral and anterior edges, becoming arch- or semicircle-shaped; T2 smooth and shiny; T2 length $0.14 \mathrm{~mm}$; T2 width at posterior edge $0.33 \mathrm{~mm}$; ovipositor slightly protruding from end of metasoma.

\section{Male \\ Unknown.}

\section{Remarks}

Glyptapanteles foraminous sp. nov. constitutes BIN: BOLD:ADL3303 and is 2.32\% (p-dist.) divergent from the closet BIN in the database (BOLD:ADZ3782, a BIN that has not been released publicly).

Using the BOLD Batch ID engine, the COI barcode of the holotype is $2.3 \%$ different from the most similar COI sequence from an Australian specimen (AUMIC274-18; Glyptapanteles eburneus sp. nov.). The type specimen was able to be sequenced for the wingless gene, which differs by a minimum of $5 \mathrm{bp}$ from all other species with available sequence data.

\section{Distribution}

This species is currently only represented by a single specimen from Cainbable Quarry in southern QLD.

Glyptapanteles fullawayi Austin \& Dangerfield, 1992

Fig. 16C

\section{Diagnosis}

The combination of a smooth propodeum, T1-2 dark, T1 only narrowing slightly posteriorly and gena without a pale spot, allow G. fullawayi to be separated from the G. albigena, G. mouldsi, G. eburneus, G. niveus and G. arcanus species groups. 
Glyptapanteles fullawayi is morphologically similar (at least using the available illustrations) to the G. austini species group and to Glyptapanteles drioplanetus. Glyptapanteles fullawayi appears to have only 6-7 large pits in the scutellar sulcus (differing from the G. austini species group and from G. drioplanetus, which all have at least 8); however, this character is prone to variability. Despite this, we feel that it is not very likely that this species is conspecific with any of the newly described species due to the distribution being restricted to Western Samoa.

\section{Material examined}

No specimens examined, illustrations and diagnosis in Austin \& Dangerfield (1992) used as reference (Fig. 16).

\section{Distribution}

Western Samoa.

Glyptapanteles goodwinnoakes Fagan-Jeffries, Bird \& Austin sp. nov. urn:lsid:zoobank.org:act:C39D8F6A-C62D-4D6C-AC74-BBEBAED50ED5

Fig. 36

\section{Diagnosis}

Glyptapanteles goodwinnoakes sp. nov. can be separated from the other members of the G. arcanus species group by having the propodeum with generally coarser sculpturing than most species other than G. erucadesolator sp. nov., G. lambkinae sp. nov., G. arcanus sp. nov. and G. vergrandiacus sp. nov. and with T1 smooth (in all four of the previously mentioned species, T1 is either rugose or at least clearly punctured in the posterior half). It is noted, however, that all these species are morphologically very similar; limited specimens were available for examination and identifications should be made or corroborated with DNA barcodes.

\section{Etymology}

This species is named for Alison Goodwin and Roger Noakes, who ran a Malaise trap on their property near Bendemeer, NSW, from which a paratype of the species originates. It is a noun in apposition.

\section{Material examined}

Holotype

AUSTRALIA - ; Queensland, Lonesome National Park; -25.495, 148.812; 585 m a.s.1.; 3-26 Nov. 2010; C. Lambkin et al. leg.; near lookout (LNP4m), closed Eucalyptus woodland on rocky ridge, Malaise trap; Extraction647, BOLD: AUMIC425-18; QM T208399.

\section{Paratypes}

AUSTRALIA - New South Wales - 1 o; Bendemeer; -30.819, 151.142; 840 m a.s.1.; 9-23 Feb. 2020; A. Goodwin and R. Noakes leg.; Malaise trap, remnant bushland on cattle-grazed property; Extraction923, BOLD: AUGLY018-21; QM T250956. - Queensland • 1 9; West Quinkan, Bush Blitz Site F3; -15.812550, 144.41974; 495 m a.s.1.; 6-15 Mar. 2017; B. Baehr leg.; open woodland; Extraction895, BOLD: AUGLY017-21; QM T250957.

\section{Description}

\section{Female}

Colouration. Gena without a pale spot; labrum mostly dark; scape colour in ventral half uniformly paler than flagellomeres; flagellomeres all black/dark brown; tegula light brown; wing veins uniformly black or brown, or with small lighter area proximally; anteromesoscutum all dark, or dark with very slight 
orange patches on posterolateral corners; scutellar disk and metanotum dark; propodeum dark; fore coxa dark; mid coxa dark; hind coxa dark; fore femur pale yellow; mid femur orange to light brown; hind femur dark reddish-brown or orange to light brown; fore tibia pale yellow; mid tibia orange to light brown; hind tibia darkening posteriorly; hind basitarsus dark reddish-brown; T1 dark; T2 sclerotised area dark; T2 lateral area same colour as sclerotised area, or only slightly paler or dark extends past indentation, but then pale; T3 dark; T4+ dark.

Holotype Body MeASUREMENTs. Body length $2.2 \mathrm{~mm}$; fore wing length $2.1 \mathrm{~mm}$; antennal length similar to body length.

HEAD. Antennal flagellomere 14 length/width 1.50-1.66; antennal flagellomere 2 length/width $2.62-$ 3.50; OOD/POD 1.71-2.00; IOD/POD 1.43-1.67.

Mesosoma. Anteromesoscutum sculpturing with shallow to deep punctures, space between punctures a mixture of smaller than diameter of punctures and of similar size. Often smoother in posterior centre; scutellar disk sculpturing with shallow punctures scattered over most of area; 8-10 pits in scutellar sulcus; propodeum with median carina absent and propodeum strongly rugose, coarse sculpturing over most of propodeum, sometimes sculpturing clearly deeper in a ' $v$ ' shape with smooth anterior centre area.

Wings. Pterostigma length $0.46 \mathrm{~mm}$; pterostigma width $0.15 \mathrm{~mm}$; r $0.17 \mathrm{~mm} ; 2 \mathrm{RS} 0.12 \mathrm{~mm} ; 2 \mathrm{~m}$ $0.07 \mathrm{~mm}$; (RS+M)b $0.08 \mathrm{~mm}$.

Metasoma. T1 lateral edges parallel for anterior $1 \frac{1}{2}$ to $2 / 3$ of length, then narrowing posteriorly; T1 smooth and shiny, a few scattered punctures; T1 length $0.34 \mathrm{~mm}$; T1 width at posterior edge $0.11 \mathrm{~mm}$; T2 an isosceles trapezoid, lateral edges straight; T2 smooth and shiny; T2 length $0.17 \mathrm{~mm}$; T2 width at posterior edge $0.12 \mathrm{~mm}$; ovipositor slightly protruding from end of metasoma.

\section{Male \\ Unknown.}

\section{Remarks}

The wingless barcode for this species is shared with G. doreyi sp. nov. and G. arcanus sp. nov. and differs by 1 bp from the barcodes of $G$. vergrandiacus sp. nov., G. doreyi sp. nov. (second haplotype) and G. lessardi sp. nov. This group of species is very closely related and would benefit from future, more detailed taxonomic work to ensure that they are not simply divergent populations of the same species. For this species hypothesis, we have made the decision to split them into different species based on the COI barcode sequences being $>3 \%$ divergent; however, we note that this is a hypothesis and is open to change with future work.

Glyptapanteles goodwinnoakes sp. nov. constitutes BIN BOLD:ADL3801 and is 3.69\% (p-dist.) divergent from the closet BIN in the database (BOLD:AEI4383; an undescribed lineage, from Australia).

Using the BOLD Batch ID engine, the COI sequence of the holotype is $6.1 \%$ different from the most similar COI sequence from an Australian specimen (AUGLY141-21; an undescribed lineage, with a single specimen).

\section{Distribution}

This species has a very disjunct distribution, found in northern QLD and in central NSW. This likely reflects poor sampling rather than the true species distribution. 


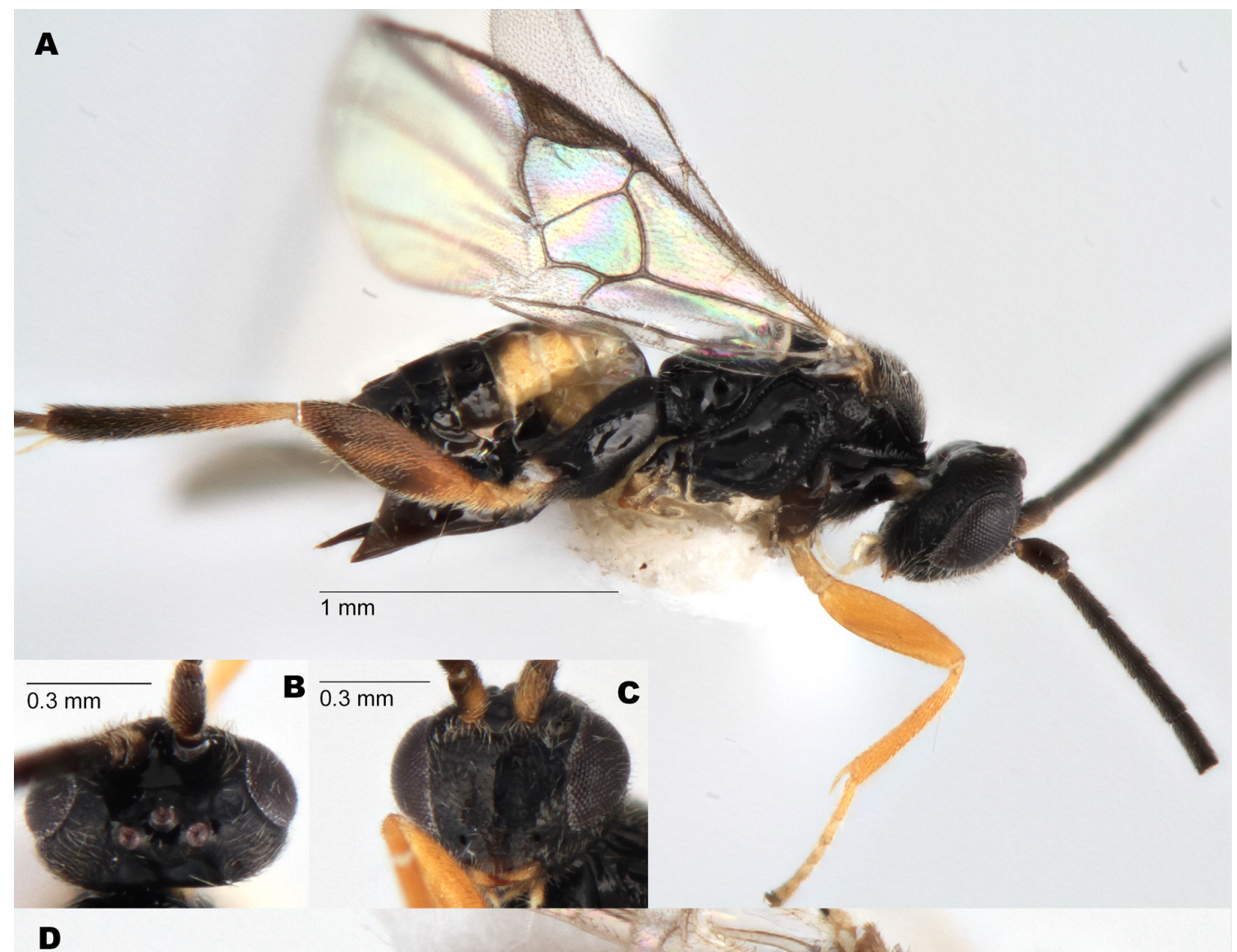

D

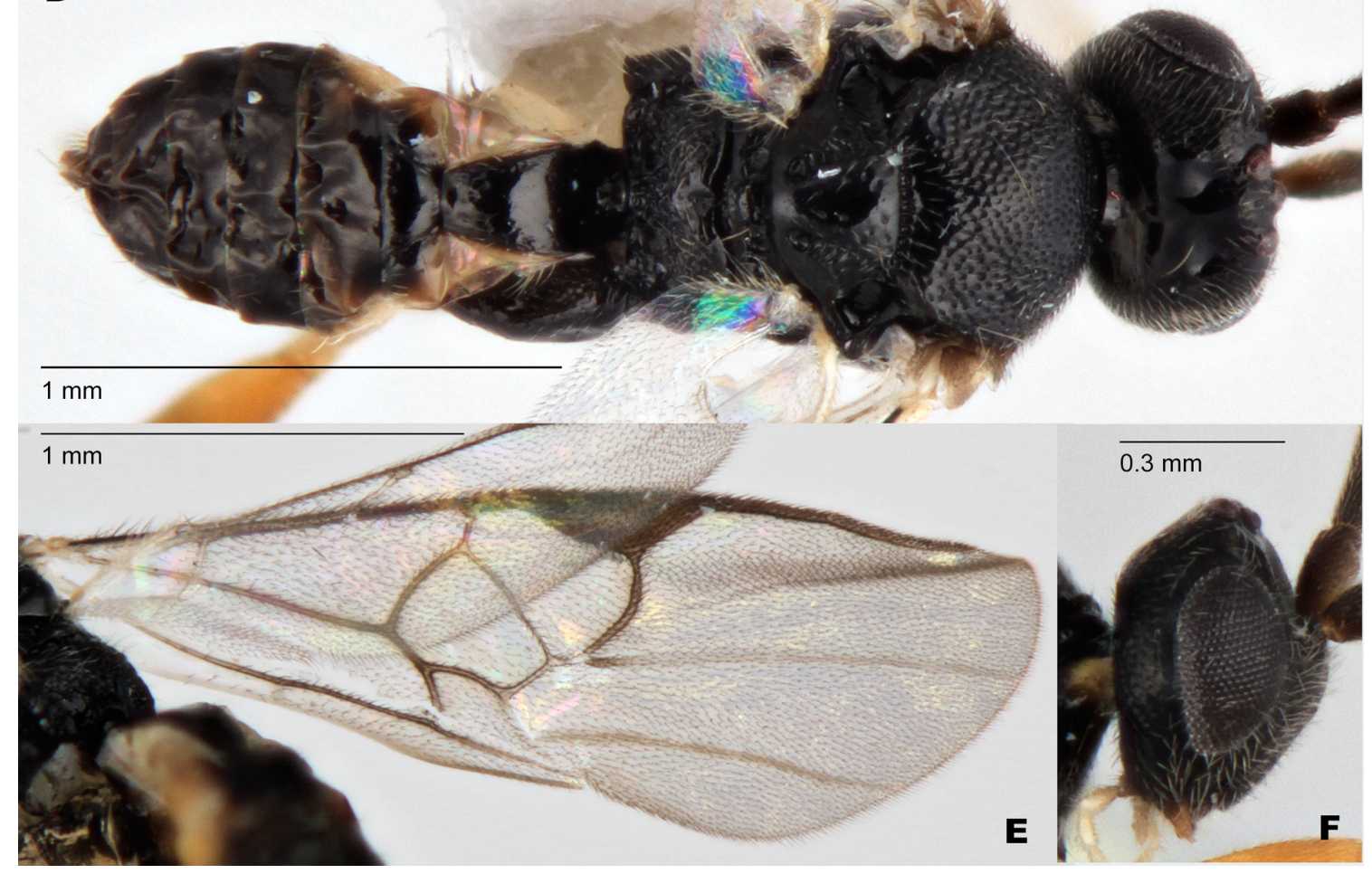

Fig. 36. Glyptapanteles goodwinnoakes Fagan-Jeffries, Bird \& Austin sp. nov., paratype, $q$ (QM T250956). A. Lateral habitus. B. Dorsal head. C. Anterior head. D. Dorsal habitus. E. Fore wing. F. Lateral head. 
Glyptapanteles guzikae Fagan-Jeffries, Bird \& Austin sp. nov. urn:lsid:zoobank.org:act:A1FFF18B-61D9-4839-B54B-E936F756125D

Fig. 37

\section{Diagnosis}

Despite the large molecular divergence in both the COI and wingless genes (see remarks section), the only morphological character found to differentiate G. guzikae sp. nov. from G. kingae sp. nov. and G. austini sp. nov. is the femur being pale with a dark area posteriorly, rather than the femur being all dark as in G. kingae sp. nov. and G. austini sp. nov. However, we note that this subtle colour character may not be robust when further specimens are added to the dataset.

\section{Etymology}

Named for Dr Michelle Guzik, who has provided many years of mentorship and advice to EPF-J.

\section{Material examined}

\section{Holotype}

AUSTRALIA • ; ; South Australia, Kangaroo Island; -35.7533, 137.321; 17-24 Mar. 2011; G. Taylor, E. Kinnaird and R. Kittel leg.; Malaise trap MT3; Extraction97, BOLD: AUMIC524-18; SAMA 3246153.

\section{Description}

\section{Female}

Colouration. Gena without a pale spot; labrum mostly dark; scape colour in ventral half the same colour or darker than flagellomeres; flagellomeres all black/dark brown; tegula pale; wing veins uniformly black or brown, or with small lighter area proximally; anteromesoscutum all dark; scutellar disk and metanotum dark; propodeum dark; fore coxa pale yellow; mid coxa pale yellow; hind coxa dark; mid femur orange to light brown; hind femur orange to light brown; mid tibia orange to light brown; hind tibia darkening posteriorly; hind basitarsus light brown; T1 dark; T2 sclerotised area dark; T2 lateral area same colour as sclerotised area, or only slightly paler; T3 mostly pale with darker patch in centre; T4+ reddish-brown.

Holotype Body MEASUREMENTs. Body length $2.1 \mathrm{~mm}$; fore wing length $2.3 \mathrm{~mm}$; antennal length slightly longer than body length.

HEAD. Antennal flagellomere 14 length/width 2.00; antennal flagellomere 2 length/width 2.57; OOD/ POD 2.17; IOD/POD 1.83.

Mesosoma. Anteromesoscutum sculpturing with shallow to deep punctures, space between punctures a mixture of smaller than diameter of punctures and of similar size, smoother in posterior centre; scutellar disk sculpturing with only very shallow punctures, smooth and shiny; nine pits in scutellar sulcus; propodeum with median carina absent, very smooth and shiny, only very shallow punctures associated with setae.

WINGS. Pterostigma length $0.51 \mathrm{~mm}$; pterostigma width $0.19 \mathrm{~mm} ; \mathrm{r} 0.13 \mathrm{~mm} ; 2 \mathrm{RS} 0.14 \mathrm{~mm} ; 2 \mathrm{~m} 0.07 \mathrm{~mm}$; (RS+M)b $0.08 \mathrm{~mm}$.

Metasoma. T1 lateral edges parallel for entirety of length, posterior corners sometimes rounded at boundary with T2; T1 mostly smooth, some punctures in posterior half or smooth in anterior half, indistinct sculpturing in posterior half; T1 length $0.34 \mathrm{~mm}$; T1 width at posterior edge $0.08 \mathrm{~mm}$; T2 


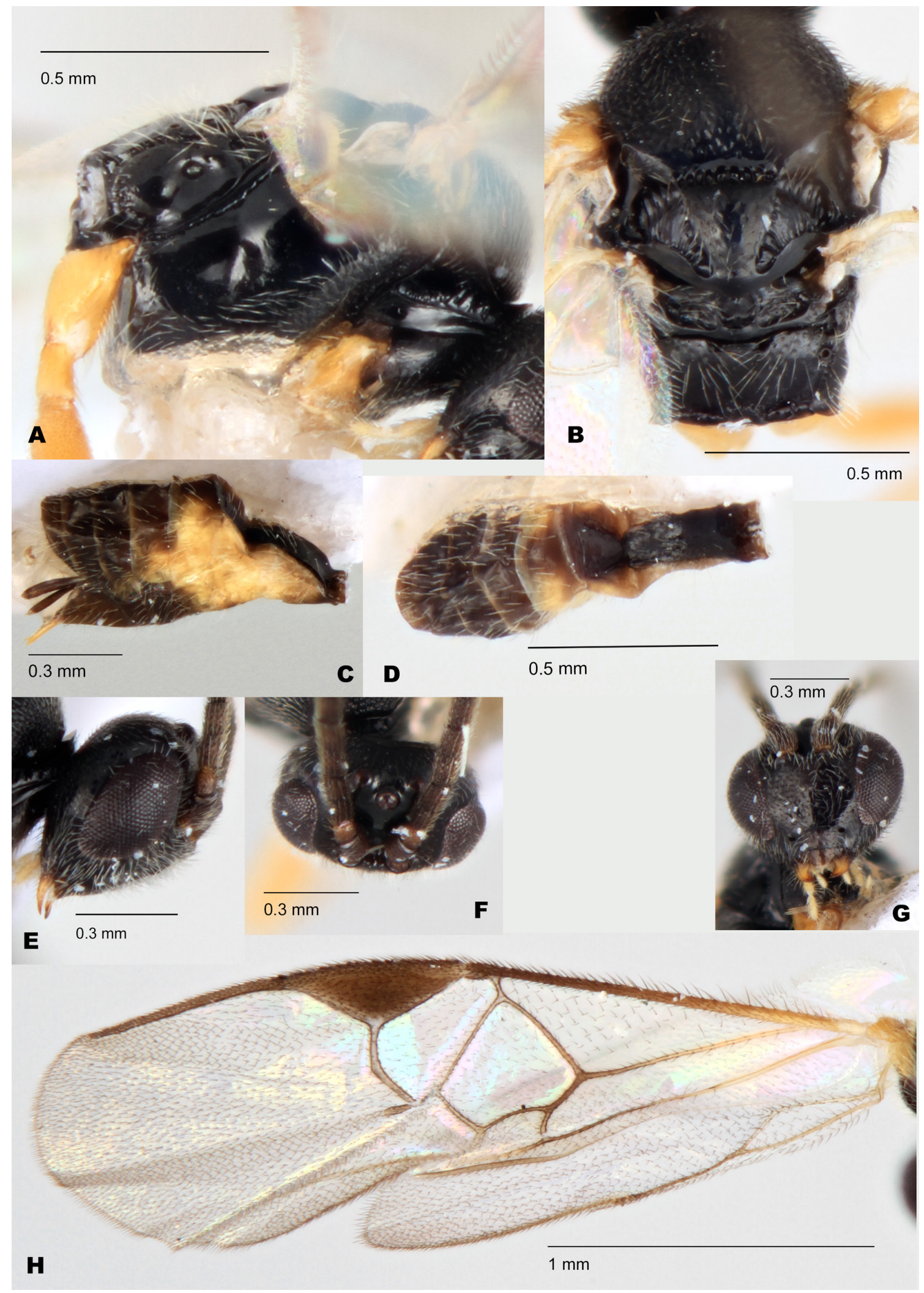

Fig. 37. Glyptapanteles guzikae Fagan-Jeffries, Bird \& Austin sp. nov., holotype, + (SAMA 32-46153). A. Lateral mesosoma. B. Dorsal mesosoma. C. Lateral metasoma. D. Dorsal metasoma. E. Lateral head. F. Dorsal head. G. Anterior head. H. Fore wing. 
an isosceles trapezoid, lateral edges straight; T2 smooth and shiny; T2 length $0.12 \mathrm{~mm}$; T2 width at posterior edge $0.14 \mathrm{~mm}$; ovipositor slightly protruding from end of metasoma.

\section{Male \\ Unknown.}

\section{Remarks}

Glyptapanteles guzikae sp. nov. constitutes BIN BOLD:ADL2952 and is 6.86\% (p-dist.) divergent from the closet BIN in the database (BOLD:ACL9923; an undescribed lineage, from New Zealand.

Using the BOLD Batch ID engine, the COI barcode of the holotype is $7.7 \%$ different from the most similar COI sequence from an Australian specimen (AUMIC291-18; Glyptapanteles austini sp. nov.). The type specimen was sequenced for the wingless gene and has a unique wingless barcode which differs by a minimum of $15 \mathrm{bp}$ from all other species with available sequence data.

\section{Distribution}

This species is known from Kangaroo Island, off the coast of SA.

Glyptapanteles harveyi Fagan-Jeffries, Bird \& Austin sp. nov. urn:1sid:zoobank.org:act:2217AE78-E98A-4283-97B4-033E2175309C

Figs 5D, 6F, 7A, 38

\section{Diagnosis}

Glyptapanteles harveyi sp. nov. is in the G. albigena species group and can be separated all other members of the species group by having the pale spot on the gena barely visible, T2 dark and the propodeum with the median carina faintly indicated at anterior and posterior ends.

\section{Etymology}

Named for Dr Mark Harvey, who collected the type material and has generously run Malaise traps to collect microgastrine material, along with providing advice for many years on the International Code of Zoological Nomenclature to EPF-J. We thank Mark for his dedication to robust taxonomic science and his support of early career researchers.

\section{Material examined}

\section{Holotype}

AUSTRALIA - O; Western Australia, Gleneagle State Forest; -32.2711, 116.163; 8-9 Oct. 2005; M.S. Harvey leg.; Malaise trap; Extraction119, BOLD: AUMIC021-18; WAM E109888.

\section{Paratype}

AUSTRALIA - 1 क; same collection data as for holotype; Extraction413, BOLD: AUMIC246-18; WAM E109889.

\section{Description}

\section{Female}

Colouration. Gena with a pale spot, but only weakly visible; labrum reddish-brown; scape colour in ventral half the same colour or darker than flagellomeres; flagellomeres all black/dark brown; tegula pale; wing veins uniformly black or brown, or with small lighter area proximally; anteromesoscutum all dark; scutellar disk and metanotum dark; propodeum dark; fore coxa dark; mid coxa dark; hind coxa dark; fore femur orange to light brown; mid femur orange to light brown; hind femur orange to light 


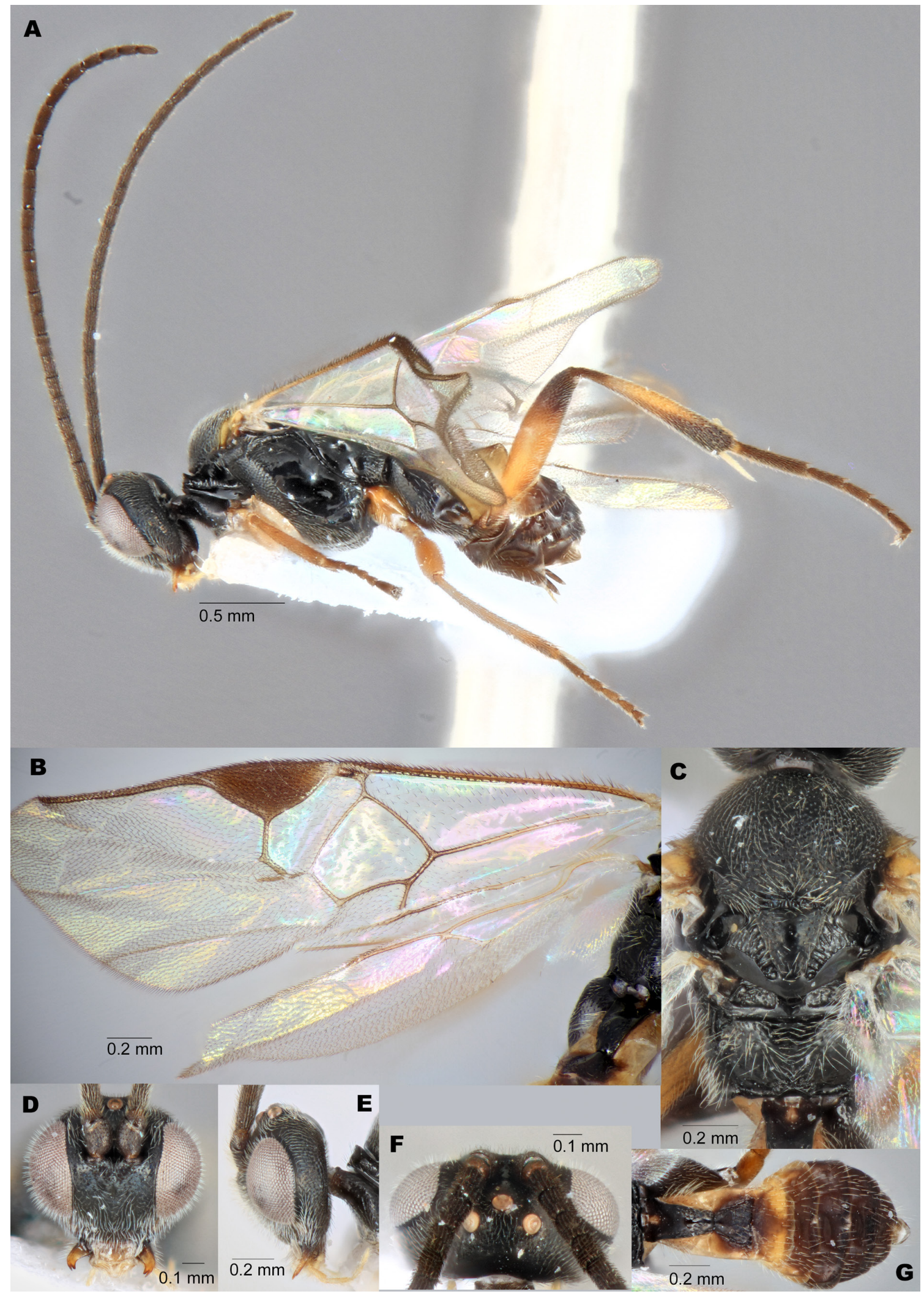

Fig. 38. Glyptapanteles harveyi Fagan-Jeffries, Bird \& Austin sp. nov. A-B, D. Holotype, $q$ (WAM E109888). C, E-G. Paratype, + (WAM E109889). A. Lateral habitus. B. Fore wing. C. Dorsal mesosoma. D. Anterior head. E. Lateral head. F. Dorsal head. G. Dorsal metasoma. 
brown; fore tibia orange to light brown; mid tibia orange to light brown; hind tibia darkening posteriorly; hind basitarsus dark reddish-brown; T1 dark; T2 sclerotised area dark; T2 lateral area dark extending past indentation, but then pale; T3 mostly pale with darker patch in centre or uniformly brown; T4+ reddish-brown.

Holotype BODY MEASUREMENTS. Body length $2.5 \mathrm{~mm}$; fore wing length $2.6 \mathrm{~mm}$; antennal length slightly longer than body length.

HEAD. Antennal flagellomere 14 length/width 2.16-2.4; antennal flagellomere 2 length/width 3.28-3.57; OOD/POD 1.86; IOD/POD 1.71-1.86.

Mesosoma. Anteromesoscutum sculpturing with shallow punctures, space between punctures generally smaller than diameter of punctures; scutellar disk sculpturing with only very shallow punctures; seven pits in scutellar sulcus; propodeum with median carina very faintly indicated in posterior half, anterior half with some punctures, postero-lateral corners smooth and shiny.

WINGS. Pterostigma length $0.63 \mathrm{~mm}$; pterostigma width $0.26 \mathrm{~mm} ; \mathrm{r} 0.14 \mathrm{~mm} ; 2 \mathrm{RS} 0.1 \mathrm{~mm} ; 2 \mathrm{~m} 0.11 \mathrm{~mm}$; $(\mathrm{RS}+\mathrm{M}) \mathrm{b} 0.1 \mathrm{~mm}$.

Metasoma. T1 wedge-shaped, narrowing posteriorly for entirety of length, lateral edges straight (but not parallel); T1 mostly smooth, some punctures in posterior half; T1 length $0.37 \mathrm{~mm}$; T1 width at posterior edge $0.07 \mathrm{~mm}$; T2 an isosceles trapezoid, lateral edges straight; 2 smooth, some very shallow punctures in posterior half; T2 length $0.16 \mathrm{~mm}$; T2 width at posterior edge $0.25 \mathrm{~mm}$; ovipositor slightly protruding from end of metasoma.

\section{Male \\ Unknown.}

\section{Remarks}

Glyptapanteles harveyi sp. nov. constitutes BIN: BOLD:ADL3315 and is 5.33\% (p-dist.) divergent from the closet BIN in the database (BOLD:ACL9711; Glyptapanteles sp. from New Zealand).

Using the BOLD Batch ID engine, the COI barcode of the holotype is $6.2 \%$ different from the most similar COI sequence from an Australian specimen (AUMIC482-18; Glyptapanteles aspersus sp. nov.). The two specimens were sequenced for the wingless gene and share a unique barcode, which differs by a minimum of $3 \mathrm{bp}$ from all other species with available sequence data.

\section{Distribution}

This species is currently known from Gleneagle State Forest near Perth, WA.

Glyptapanteles kingae Fagan-Jeffries, Bird \& Austin sp. nov. urn:1sid:zoobank.org:act:9FC2A388-3A78-47E5-99BE-789CD56291CE

Figs 14B, 39

\section{Diagnosis}

Glyptapanteles kingae sp. nov. is in the G. austini species group. Despite the large molecular divergence in both the COI and wingless genes (see Remarks section), the only morphological character found to differentiate G. kingae sp. nov. from G. guzikae sp. nov. is the femur being completely dark (G. guzikae sp. nov. has the femur pale with a dark area posteriorly). Glyptapanteles kingae sp. nov. can be separated from $G$. austini sp. nov. as follows: G. kingae sp. nov. has the propodeum with stronger sculpturing in 
the centre, whilst G. austini sp. nov. has the propodeum smoother in the centre. However, we note that there are limited specimens available and the variability of propodeal sculpturing could broaden as more populations of these species are identified and we recommend confirming identifications with DNA barcodes.

\section{Etymology}

This species is named for Dr Rachael King, who first introduced the concepts of taxonomy and systematics to EPF-J during an honours project on amphipods in the Pilbara. Fittingly, this species is the only member of Glyptapanteles currently described from the Pilbara region.

\section{Material examined}

\section{Holotype}

AUSTRALIA • +; South Australia, Witchelina Stn; -30.1211, 137.923; 19 Oct. 2010; F. Colombo leg.; Bush Blitz Svy FC055 sweeping; Extraction508, BOLD: AUMIC309-18; SAMA 32-46154.

\section{Paratypes}

AUSTRALIA - Australian Capital Territory • 1 q; Black Mountain Res, W side; -35.2764, 149.0897; 610 m a.s.1.; 31 Mar. 2020; K.M. Bayless leg.; sweep over grass, rocks and Callistemon; Extraction1591, BOLD: AUGLY113-21; ANIC 32 130331. - South Australia • 1 क; Witchelina Stn; -30.1853, 137.977; 18-22 Oct. 2010; S. Mantel, F. Colombo and R. Kittel leg.; Bush Blitz Svy, Malaise 2 in dry creek bed [with] Eremophila freelingi and Acacia tetragonophila; Extraction22, BOLD: AUMIC101-18; SAMA 32-46155. - Western Australia • 1 § ; Western Pilbara, Hamersley Stn, Nanutarra-Wittenoom Rd., approx. 13 km NE of Railway Rd. crossing, near fence line; -22.435556, 117.832222; 22-27 Sep. 2005; Conservation Volunteers Australia leg.; Extraction1354, BOLD: AUGLY080-21; AM K.247607.

\section{Description}

\section{Female}

Colouration. Gena without a pale spot; labrum mostly dark; scape colour in ventral half same colour as flagellomeres; flagellomeres all dark; tegula pale; wing veins uniformly dark, or with small lighter area proximally; anteromesoscutum all dark; scutellar disk and metanotum dark; propodeum dark; fore coxa dark; mid coxa dark; hind coxa dark; fore femur orange to light brown; mid femur orange to light brown; hind femur dark; fore tibia orange to light brown; mid tibia orange to light brown; hind tibia darkening posteriorly; hind basitarsus dark; T1 dark; T2 sclerotised area black; T2 lateral area same colour as sclerotised area, or only slightly paler, or dark extends past indentation, but then pale; T3 dark; T4+ dark.

Body MeASUREMENTs. Body length $2.0 \mathrm{~mm}$; fore wing length $2.2 \mathrm{~mm}$; antennal length slightly longer than body length.

HEAD. Face with fine punctures associated with setae; flagellomere 14 length/width 1.43-1.83; flagellomere 2 length/width 2.86-3.80; OOD/POD 1.86-2.00; IOD/POD 1.43.

Mesosoma. Anteromesoscutum sculpturing with shallow punctures, space between punctures generally smaller than diameter of punctures, slightly denser and deeper punctures anteriorly, smoother in posterior centre; scutellar disk sculpturing with only very shallow punctures; 8-11 pits in scutellar sulcus; propodeum with median carina absent and indistinct sculpturing present in centre, with rest of propodeum smooth, or propodeum with median carina present in posterior third, with indistinct sculpturing present in centre, with rest of propodeum smooth.

Wings. Pterostigma length $0.5 \mathrm{~mm}$; pterostigma width $0.17 \mathrm{~mm}$; $0.13 \mathrm{~mm} ; 2 \mathrm{RS} 0.14 \mathrm{~mm} ; 2 \mathrm{~m} 0.07 \mathrm{~mm}$; $(\mathrm{RS}+\mathrm{M}) \mathrm{b} 0.08 \mathrm{~mm}$. 


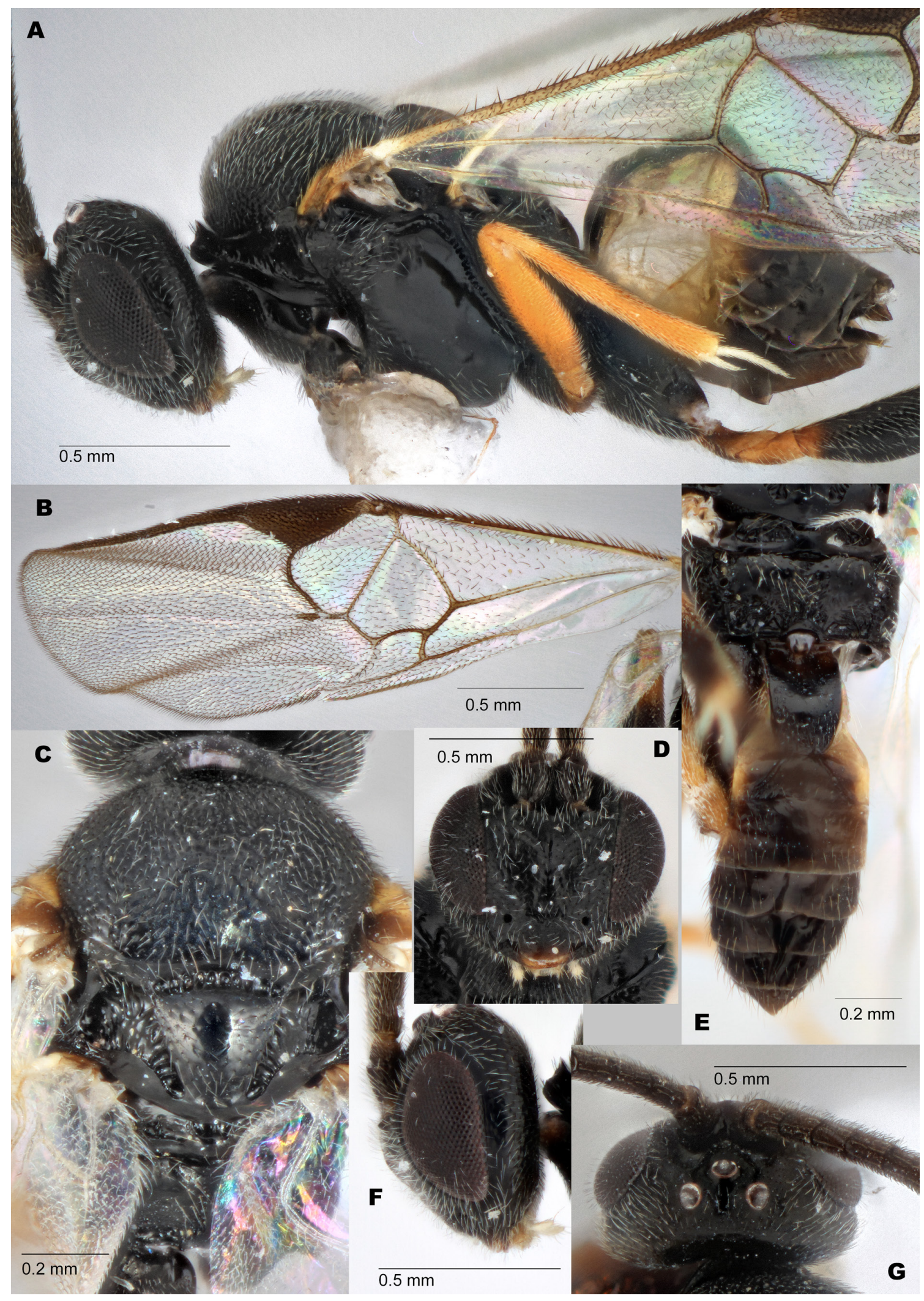

Fig. 39. Glyptapanteles kingae Fagan-Jeffries, Bird \& Austin sp. nov. A, C-D, F. Paratype, $q$ (SAMA 32-46155). B, E, G. Holotype, q (SAMA 32-46154) A. Lateral mesosoma. B. Fore wing. C. Dorsal mesosoma. D. Anterior head. E. Dorsal metasoma. F. Lateral head. G. Dorsal head. 
Metasoma. T1 broadest at centre of length, narrowing both posteriorly and anteriorly from mid-point; T1 smooth; T1 length $0.31 \mathrm{~mm}$; T1 width at posterior edge $0.11 \mathrm{~mm}$; T2 an isosceles trapezoid, lateral edges straight; $\mathrm{T} 2$ smooth and shiny; T2 length $0.14 \mathrm{~mm}$; T2 width at posterior edge $0.23 \mathrm{~mm}$; ovipositor slightly protruding from end of metasoma.

Male

As female, eight pits in scutellar sulcus.

\section{Remarks}

Glyptapanteles kingae sp. nov. constitutes BIN BOLD:ADL5629 and is 9.94\% (p-dist.) divergent from the closet BIN in the database (BOLD:ADL2952; Glyptapanteles guzikae sp. nov.).

Using the BOLD Batch ID engine, the COI barcode of the holotype is $10.1 \%$ different from the most similar COI sequence from an Australian specimen (AUMIC524-18; Glyptapanteles guzikae sp. nov.). All four specimens of the type series were sequenced for the wingless gene, which is identical amongst all specimens (although there is a single ambiguity in the WA specimen sequence). The wingless sequences differ by a minimum of $19 \mathrm{bp}$ from all other species with available sequence data.

\section{Distribution}

This species has a wide distribution, from the Pilbara in WA, through to the arid region of SA and in the $\mathrm{ACT}$ at Black Mountain Reserve.

Glyptapanteles kittelae Fagan-Jeffries, Bird \& Austin sp. nov. urn:lsid:zoobank.org:act:97C25961-9321-4C68-83FD-3563AADE8B60

Figs 5C, 7B, 8B, 9B, 40

\section{Diagnosis}

Glyptapanteles kittelae sp. nov. is in the G. albigena species group and can be separated from most of the other members of the species group by the pale spot on the gena being small but clearly visible, T2 dark, the media carina completely absent, and the hind femur light brown to pale.

With the limited specimens available, there was not a morphological character found that easily differentiates G. kittelae sp. nov. from G. austrinus sp. nov. or G. aspersus sp. nov. The COI divergence between G. kittelae sp. nov. and G. austrinus sp. nov. is $>6 \%$, whilst the divergence between G. kittelae sp. nov. and G. aspersus sp. nov. is $>7 \%$. The wingless sequences of G. kittelae sp. nov. and G. aspersus sp. nov. are very distinct, differing by 7 bp; however, there are no wingless sequences available for $G$. austrinus sp. nov.

\section{Etymology}

This species is named for Dr Rebecca Kittel, who collected the holotype and many other important and useful Hymenoptera, including many specimens of microgastrines, during her time studying Australian Cheloninae.

\section{Material examined}

\section{Holotype}

AUSTRALIA • क; South Australia, Witchelina Stn; -30.0186, 137.901; 23 Oct. 2010; R. Kittel leg.; Bush Blitz Svy RK091 sweeping Acacia victoriae; Extraction557, BOLD: AUMIC348-18; SAMA 32-46156.

\section{Paratype}

AUSTRALIA • 1 \%; New South Wales, track off Oxley Highway, $15 \mathrm{~km}$ from Gingers Creek, roadside; $-31.3655,152.045278 ; 1116$ m a.s.1.; 15 Dec. 2008; K. Harvey and K. Umbers; reared from flowers of Senecio minimus 1 Apr. 2009; Extraction1313, BOLD: AUGLY152-21; AM K379892. 


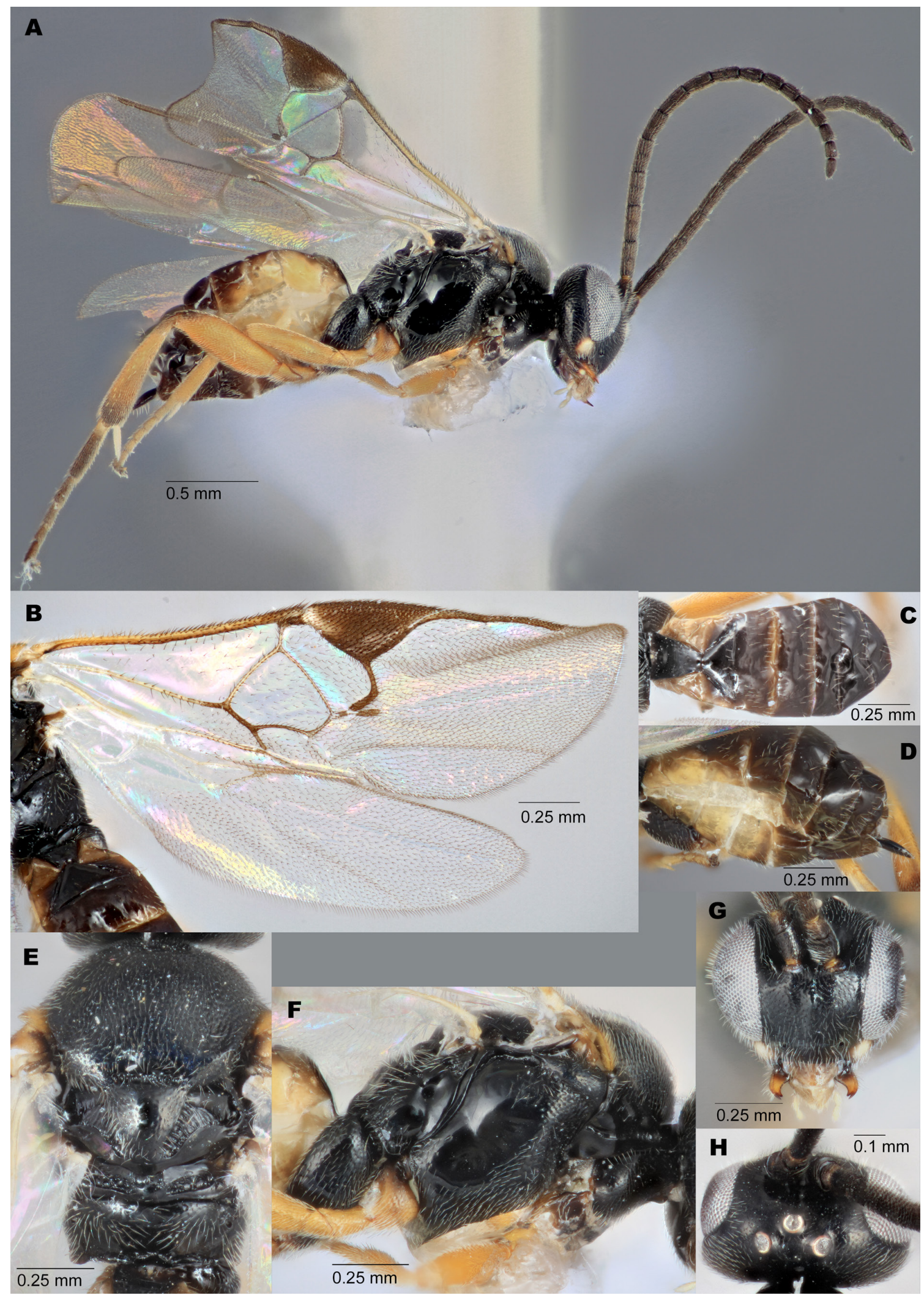

Fig. 40. Glyptapanteles kittelae Fagan-Jeffries, Bird \& Austin sp. nov., holotype, $q$ (SAMA 3246156). A. Lateral habitus. B. Fore wing. C. Dorsal metasoma. D. Lateral metasoma. E. Dorsal mesosoma. F. Lateral mesosoma. G. Anterior head. H. Dorsal head. 


\section{Description}

\section{Female}

Colouration. Gena with a pale spot; labrum reddish-brown; scape colour in ventral half the same colour or darker than flagellomeres; flagellomeres all black/dark brown; tegula pale; wing veins uniformly black or brown, or with small lighter area proximally or uniformly pale; anteromesoscutum all dark; scutellar disk and metanotum dark; propodeum dark; fore coxa dark; mid coxa light brown; hind coxa dark; fore femur orange to light brown; mid femur orange to light brown; hind femur orange to light brown; fore tibia orange to light brown; mid tibia orange to light brown; hind tibia darkening posteriorly; hind basitarsus dark reddish-brown; T1 dark; T2 sclerotised area dark; T2 lateral area same colour as sclerotised area, or only slightly paler; T3 uniformly brown; T4+ reddish-brown.

Holotype Body MEASUREMENTS. Body length $2.2 \mathrm{~mm}$; fore wing length $2.1 \mathrm{~mm}$; antennal length slightly shorter than body length.

HeAD. Antennal flagellomere 14 length/width 1.12-1.40; antennal flagellomere 2 length/width $2.42-$ 3.00; OOD/POD 1.71; IOD/POD 1.71.

Mesosoma. Anteromesoscutum sculpturing with shallow punctures, space between punctures generally smaller than diameter of punctures; scutellar disk sculpturing with only very shallow punctures; eight pits in scutellar sulcus; propodeum with median carina absent, very smooth and shiny, only very shallow punctures associated with setae.

WINGS. Pterostigma length $0.51 \mathrm{~mm}$; pterostigma width $0.22 \mathrm{~mm} ; \mathrm{r} 0.13 \mathrm{~mm} ; 2 \mathrm{RS} 0.14 \mathrm{~mm} ; 2 \mathrm{~m} 0.1 \mathrm{~mm}$; $(\mathrm{RS}+\mathrm{M}) \mathrm{b} 0.07 \mathrm{~mm}$.

Metasoma. T1 wedge-shaped, narrowing posteriorly for entirety of length, lateral edges straight (but not parallel); T1 smooth and shiny; T1 length $0.31 \mathrm{~mm}$; T1 width at posterior edge $0.06 \mathrm{~mm}$; T2 an isosceles trapezoid, lateral edges straight; T2 smooth and shiny; T2 length $0.14 \mathrm{~mm}$; T2 width at posterior edge $0.31 \mathrm{~mm}$; ovipositor slightly protruding from end of metasoma.

\section{Male \\ Unknown.}

\section{Remarks}

Glyptapanteles kittelae sp. nov. constitutes BIN: BOLD:ADL3407 and is 5.13\% (p-dist.) divergent from the closet BIN in the database (BOLD:ADL4089; Glyptapanteles ferrugineus sp. nov.).

Using the BOLD Batch ID engine, the COI barcode of the holotype is $5.3 \%$ different from the most similar COI sequence from an Australian specimen (AUMIC042-18; Glyptapanteles ferrugineus sp. nov.). The holotype was able to be sequenced for the wingless gene and has a unique barcode, which differs by a minimum of $5 \mathrm{bp}$ from all other species with available sequence data.

\section{Distribution}

Glyptapanteles kittelae sp. nov. is known from central SA and eastern NSW.

Glyptapanteles kurandaensis Fagan-Jeffries, Bird \& Austin sp. nov. urn:1sid:zoobank.org:act:14DC77FB-9FEB-4DE4-8D02-4678EBB481E5

Figs 12D, 14A, 41

\section{Diagnosis}

Glyptapanteles kurandaensis sp. nov. can be separated from the other species of Glyptapanteles described from Australia by having the gena without a pale spot, T1 and T2 dark, anteromesoscutum 
punctures not extremely sparse and deep (i.e., not as in the G. niveus species group), no orange markings on the postero-lateral anteromesoscutum, propodeum without strong rugose sculpturing (sometimes with shallow sculpturing in centre, but never over most of propodeum), hind femur solidly dark in colouration, ventral side of antennal scape (at least in distal half) the same colour or darker than the flagellomeres, and fore wing veins $r$ and 2SR narrow and smoothly joined, both significantly longer than vein $2 \mathrm{~m}$ (compared to the sharply angled, thicker and shorter $r$ and 2SR veins more typical of this genus in Australia).

\section{Etymology}

Named for the collection locality, Kuranda, a small town near Cairns in northern QLD.

\section{Material examined}

Holotype

AUSTRALIA • + ; Queensland, Kuranda; -16.8135; 145.6430586; 317 m a.s.1.; 12 Feb.-6 Apr. 2020; M.S. Moulds leg.; Malaise Trap EFJ2020MT36; Extraction1568, BOLD: AUGLY110-21; QM T250958.

\section{Paratypes}

AUSTRALIA - Queensland - 1 ; ; same collection data as for holotype: Extraction1520, BOLD: AUGLY092-21; QM T250959 - 1 क; same collection data as for holotype; Extraction1542, BOLD: AUGLY098-21; QM T250960 - 1 O ; same collection data as for holotype; Extraction1543, BOLD: AUGLY099-21; QM T250961 • 1 क (ethanol); same collection data as for holotype; Extraction1546, BOLD: AUGLY101-21; QM T250962 - 1 क (ethanol); same collection data as for holotype; Extraction1551, BOLD: AUGLY103-21; QM T250963 • 1 क ; same collection data as for holotype; Extraction1554, BOLD: AUGLY104-21; QM T250964 • 1 क (ethanol); same collection data as for holotype; Extraction1565, BOLD: AUGLY109-21; QM T250965 • 1 क; same collection data as for holotype; Extraction1569, BOLD: AUGLY111-21; QM T250966 • 1 क ; same collection data as for holotype; Extraction1570, BOLD: AUGLY112-21; QM T250967 • 1 क; same collection data as for holotype; Extraction1508, BOLD: AUGLY129-21; QM T250968 • 1 क ; same collection data as for holotype; Extraction296, BOLD: AUMIC161-18; QM T250969 • 1 \& (ethanol); same collection data as for holotype; 10 Feb.-15 Mar. 2017; Extraction500, BOLD: AUMIC306-18; QM T250970 • 1 क ; same collection data as for holotype; 16 Mar.-12 Apr. 2017; Extraction551, BOLD: AUMIC342-18; QM T250971.

\section{Description}

\section{Female}

Colouration. Gena without a pale spot; labrum mostly dark or reddish-brown; scape colour in ventral half the same colour or darker than flagellomeres; flagellomeres darkening distally; tegula dark; wing veins uniformly black or brown, or with small lighter area proximally; anteromesoscutum all dark; scutellar disk and metanotum dark; propodeum dark; fore coxa dark; mid coxa dark; hind coxa dark; fore femur pale yellow; mid femur pale yellow; hind femur dark reddish-brown or dark; fore tibia pale yellow; mid tibia pale yellow; hind tibia darkening posteriorly; hind basitarsus light brown; T1 dark; T2 sclerotised area dark or dark reddish-brown; T2 lateral area same colour as sclerotised area, or only slightly paler, or dark extends past indentation, but then pale; T3 dark, mostly dark with paler lateral areas or uniformly brown; T4+ dark or reddish-brown.

Holotype Body meAsuRements. Body length $1.8 \mathrm{~mm}$; fore wing length $1.9 \mathrm{~mm}$; antennal length slightly shorter than body length.

HEAD. Antennal flagellomere 14 length/width 1.80-2.25; antennal flagellomere 2 length/width $2.42-$ 3.80; OOD/POD 1.86-2.33; IOD/POD 1.29-2.00. 


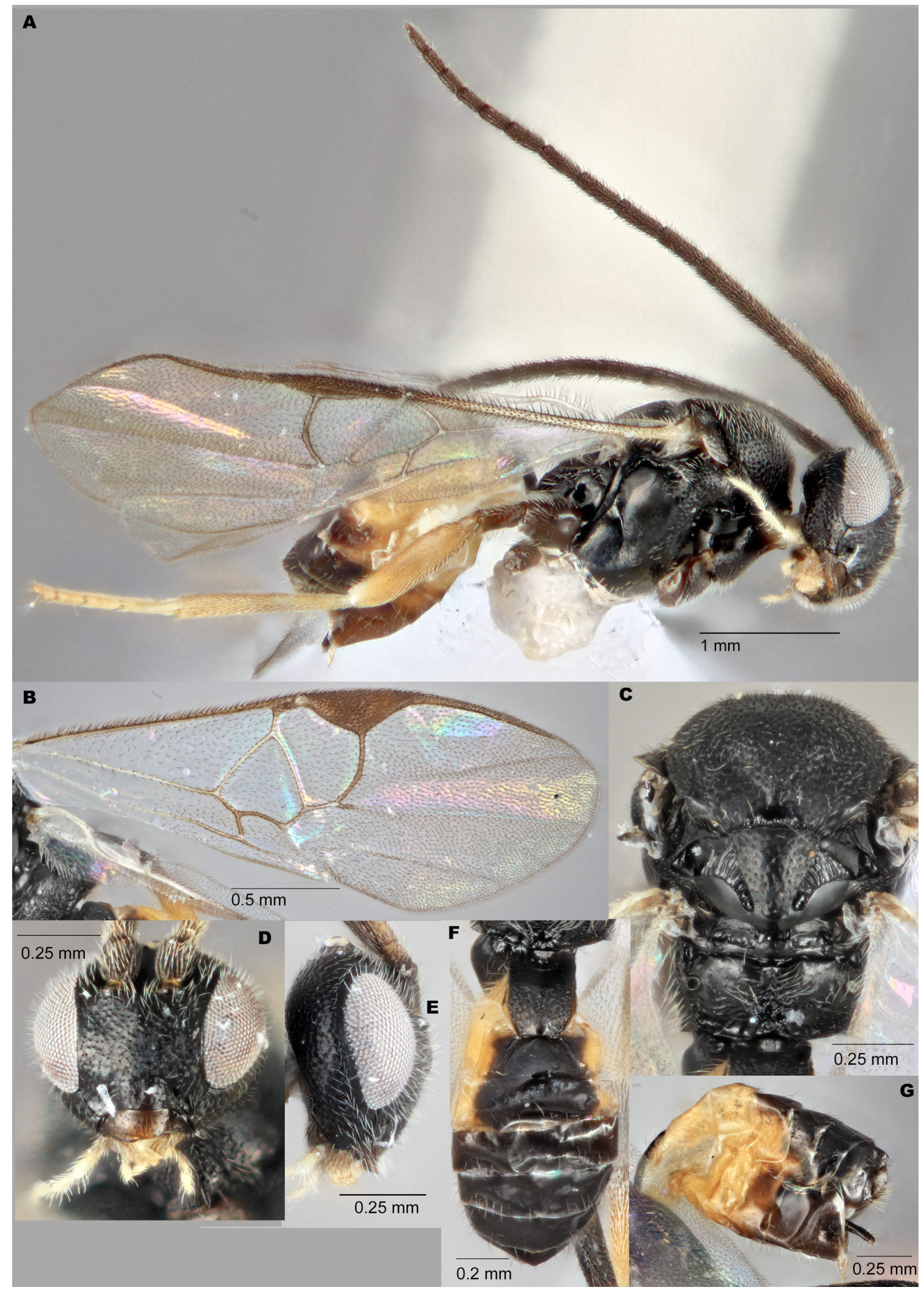

Fig. 41. Glyptapanteles kurandaensis Fagan-Jeffries, Bird \& Austin sp. nov., paratypes, ${ }$. A-B, D-E. (QM T250969). C, F-G. QM T250971. A. Lateral habitus. B. Fore wing. C. Dorsal mesosoma. D. Anterior head. E. Lateral head. F. Dorsal metasoma. G. Lateral metasoma. 
Mesosoma. Anteromesoscutum sculpturing with shallow punctures, space between punctures generally smaller than diameter of punctures, slightly denser and deeper punctures anteriorly, smoother in posterior centre; scutellar disk sculpturing with only very shallow punctures; 7-9 pits in scutellar sulcus; propodeum with median carina absent, some indistinct sculpturing in posterior centre, or in centre along length of propodeum, or median carina faintly indicated for all of length by indistinct sculpturing.

WINGs. Pterostigma length $0.48 \mathrm{~mm}$; pterostigma width $0.15 \mathrm{~mm} ; \mathrm{r} 0.17 \mathrm{~mm} ; 2 \mathrm{RS} 0.12 \mathrm{~mm} ; 2 \mathrm{~m} 0.09 \mathrm{~mm}$; (RS+M)b $0.1 \mathrm{~mm}$.

METASOMA. T1 lateral edges parallel for anterior $3 / 4$ of length, then narrowing posteriorly, or lateral edges parallel for entirety of length, posterior corners sometimes rounded at boundary with T2; T1 mostly smooth, some punctures in posterior half or mostly smooth, some shallow rugose sculpturing in posterior half; T1 length $0.29 \mathrm{~mm}$; T1 width at posterior edge $0.14 \mathrm{~mm}$; T2 an isosceles trapezoid, lateral edges straight, with curved lateral and anterior edges, becoming arch- or semicircle-shaped or almost square, lateral edges almost parallel, only broadening posteriorly very slightly; T2 smooth and shiny; T2 length $0.13 \mathrm{~mm}$; T2 width at posterior edge $0.24 \mathrm{~mm}$; ovipositor slightly protruding from end of metasoma.

\section{Male}

Unknown.

\section{Remarks}

Glyptapanteles kurandaensis sp. nov. constitutes BIN BOLD:ADL2798 and is 2.4\% (p-dist.) divergent from the closet BIN in the database (BOLD:AAH1268, Glyptapanteles creatonoti (Viereck, 1912)). We have examined the images of $G$. creatonoti in Gupta et al. (2016) and are confident that G. kurandaensis sp. nov. is a different species, most easily separated by the following morphological characteristics: G. kurandaensis sp. nov. has the hind coxa dark (G. creatonoti hind coxa pale; "stamineous" in original description); G. kurandaensis sp. nov. has the propodeum much smoother than that of G. creatonoti and G. kurandaensis sp. nov. has T1 completely black, whilst G. creatonoti has the anterior area of T1 pale ("stamineous" in original description, appearing pale orange in images of Gupta et al. (2016)).

Using the BOLD Batch ID engine, the COI barcode of the holotype is $7.7 \%$ different from the most similar COI sequence from an Australian specimen (AUMIC291-18; Glyptapanteles austini sp. nov.). The type specimen was sequenced for the wingless gene, which differs by a minimum of $14 \mathrm{bp}$ from all other species with available sequence data.

\section{Distribution}

This species is currently known from one collection site; Kuranda in north-eastern QLD.

Glyptapanteles lambkinae Fagan-Jeffries, Bird \& Austin sp. nov. urn:1sid:zoobank.org:act:AB18FD64-2820-46A6-A7CD-F3869B4CA991

Fig. 42

\section{Diagnosis}

Glyptapanteles lambkinae sp. nov. is in the G. arcanus species group and can be separated from the other members of the species group, other than from $G$ erucadesolator sp. nov., by having the propodeum with very coarse and strong rugose sculpting and T1 with strong sculpturing. Glyptapanteles arcanus sp. nov., G. vergrandiacus sp. nov. and G. goodwinnoakes sp. nov. also have coarse sculpturing on the propodeum (although to a lesser degree), but T1 is either smooth or with clear punctures (but not rugose sculpturing). It is noted, however, that all these species are morphologically very similar and identifications should be made with DNA barcodes. There was not a morphological character found to 
easily differentiate G. lambkinae sp. nov. from G. erucadesolator sp. nov., which are sister lineages in the current phylogeny (Fig. 2); however, as the species differ by $2 \mathrm{bp}$ in the wingless sequence and the COI barcodes are $>6 \%$ divergent, we feel confident that these are different species.

\section{Etymology}

Named for Dr Christine Lambkin, who collected the type material and EPF-J and ARD thank her for all her support and help over the years.

\section{Material examined}

\section{Holotype}

AUSTRALIA • O; Queensland, Lamington National Park; -28.155, 153.139; 282 m a.s.1.; 13-23 Jan. 2007; C. Lambkin and N. Starick leg.; IBISCA Plot \# IQ-300-B, rainforest, Malaise trap; bulk vial 22139; Extraction601, BOLD: AUMIC387-18; QM T208402.

\section{Paratypes}

AUSTRALIA - 1 क ; same collection data as for holotype; Extraction1467, BOLD: AUGLY085-21; QM T250972 • 1 क ; same collection data as for holotype; Extraction1471, BOLD: AUGLY089-21; QM T250973 • 1 \& (ethanol); same collection data as for holotype; Extraction599, BOLD: AUMIC383-18; QM T208401.

\section{Description}

\section{Female}

Colouration. Gena without a pale spot; labrum reddish-brown; scape colour in ventral half uniformly paler than flagellomeres; flagellomeres all black/dark brown; tegula pale; wing veins uniformly reddish-brown; anteromesoscutum all dark or dark with very slight orange patches on posterolateral corners; scutellar disk and metanotum dark; propodeum dark; fore coxa pale yellow; mid coxa pale yellow; hind coxa dark; fore femur pale yellow; mid femur pale yellow; hind femur orange to light brown; fore tibia pale yellow; mid tibia pale yellow; hind tibia darkening posteriorly; hind basitarsus dark reddish-brown; T1 dark; T2 sclerotised area dark reddish-brown; T2 lateral area dark extends past indentation, but then pale; T3 mostly pale with darker patch in centre or mostly dark with paler lateral areas; T4+ reddish-brown.

Holotype Body MEASUREMENTs. Body length $2.7 \mathrm{~mm}$; fore wing length $2.5 \mathrm{~mm}$; antennal length similar to body length.

HEAD. Antennal flagellomere 14 length/width 1.12-1.62; antennal flagellomere 2 length/width 3.00 3.85; OOD/POD 1.43-1.71; IOD/POD 1.33-1.71.

Mesosoma. Anteromesoscutum sculpturing with deep punctures, space between punctures a mixture of smaller than diameter of punctures, and of similar size; scutellar disk sculpturing with deep, sparse, irregularly spaced punctures, more common on anterior lateral edges; 10-11 pits in scutellar sulcus; propodeum with median carina absent, strongly rugose, sometimes with punctures in centre.

Wings. Pterostigma length $0.62 \mathrm{~mm}$; pterostigma width $0.21 \mathrm{~mm}$; $\mathrm{r} 0.19 \mathrm{~mm} ; 2 \mathrm{RS} 0.17 \mathrm{~mm} ; 2 \mathrm{~m} 0.11 \mathrm{~mm}$; $(\mathrm{RS}+\mathrm{M}) \mathrm{b} 0.12 \mathrm{~mm}$.

Metasoma. T1 lateral edges parallel for anterior $3 / 4$ of length, then narrowing posteriorly; T1 coarsely sculptured; T1 length $0.46 \mathrm{~mm}$; T1 width at posterior edge $0.09 \mathrm{~mm}$; T2 an isosceles trapezoid, lateral edges straight; T2 smooth and shiny; T2 length $0.16 \mathrm{~mm}$; T2 width at posterior edge $0.27 \mathrm{~mm}$; ovipositor slightly protruding from end of metasoma.

\section{Male}

Unknown. 


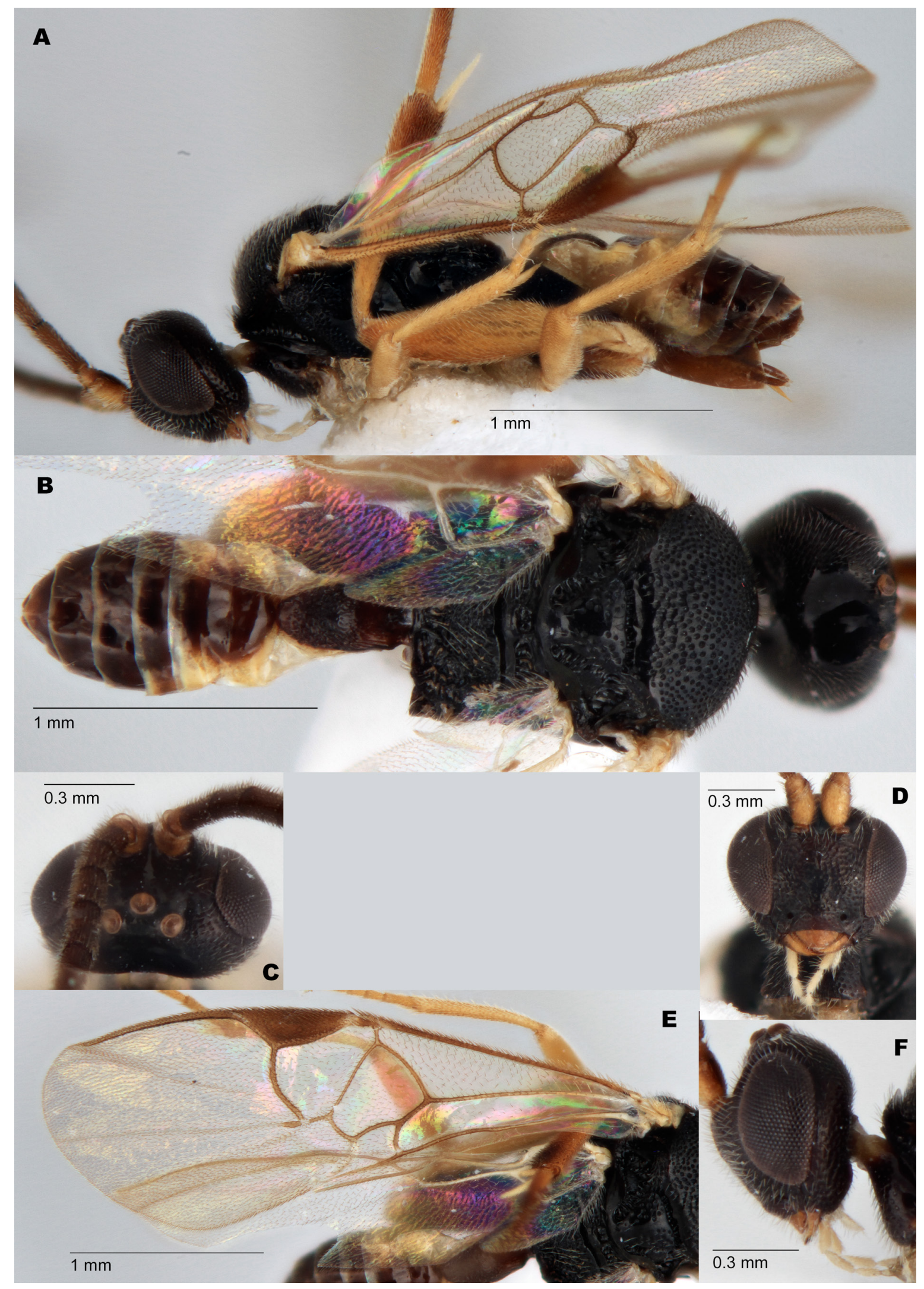

Fig. 42. Glyptapanteles lambkinae Fagan-Jeffries, Bird \& Austin sp. nov., paratype, $q$ (QM T250973). A. Lateral habitus. B. Dorsal habitus. C. Dorsal head. D. Anterior head. E. Fore wing. F. Lateral head. 


\section{Remarks}

Glyptapanteles lambkinae sp. nov. constitutes BIN BOLD:ADL3041 and is 6.09\% (p-dist.) divergent from the closet BIN in the database (BOLD:ADL3293; Glyptapanteles wrightae sp. nov.).

Using the BOLD Batch ID engine, the COI sequence of the holotype is $6.1 \%$ different from the most similar COI sequence from an Australian specimen (AUGLY141-21; an undescribed lineage, with a single specimen). All four five specimens were sequenced for the wingless gene, which is identical within the species and differs by a minimum of $2 \mathrm{bp}$ from all other species with available sequence data.

\section{Distribution}

This species is known only from Lamington National Park in southern QLD.

Glyptapanteles lessardi Fagan-Jeffries, Bird \& Austin sp. nov. urn:lsid:zoobank.org:act:3DDE9CAF-3AD2-49C2-BBD6-875FF512E6C9

Fig. 43

\section{Diagnosis}

Glyptapanteles lessardi sp. nov. is in the G. arcanus species group and can be separated from the other members of the group as follows:

Glyptapanteles lessardi sp. nov. can be separated from G. rodriguezae sp. nov. and G. ruhri sp. nov. by T1 being smooth and shiny, not having punctures that cover at least a third of the area of the posterior half of the tergite.

Glyptapanteles lessardi sp. nov. can be separated from G. goodwinnoakes sp. nov., G. erucadesolator sp. nov., G. lambkinae sp. nov., G. arcanus sp. nov. and G. vergrandiacus sp. nov. by the propodeum being less coarsely (and less consistently), strongly rugose sculptured across the anterior half of the propodeum. Glyptapanteles wrightae sp. nov. has the propodeum with strong punctures in the anterior half, the posterior half with shallow or strong rugose sculpturing.

Glyptapanteles lessardi sp. nov. can be separated from G. doreyi sp. nov. by having the tegula pale in colouration (the tegula in $G$. doreyi sp. nov. is generally dark in colouration), the hind femur mostly pale or light brown (mostly dark in G. doreyi sp. nov.) and the indentation in the centre of the mesopleuron being smooth, not strongly canaliculated as it is in G. doreyi sp. nov.

There was not a morphological character found that easily separates $G$. lessardi sp. nov. from G. wrightae sp. nov.; we do not diagnose these species morphologically. The wingless barcodes of the two species differ by 3 bp and the $C O I$ is greater than $4 \%$ divergent.

\section{Etymology}

Named for Dr Bryan Lessard, part of the team who collected the holotype, for his constant endeavour to raise awareness about the importance of taxonomy in the community and his support of other early career researchers.

\section{Material examined}

\section{Holotype}

AUSTRALIA - + ; Queensland, Blue Tongue Scrub, Steve Irwin Reserve, Cape York; -12.286584, 142.343274; 42.9 m a.s.1.; 23-26 Jul. 2018; C. Lambkin and B. Lessard leg.; vine thicket rainforest; Extraction888, BOLD: AUGLY015-21; QM T250974. 
Paratype

AUSTRALIA - 1 क; same collection data as for preceding; Extraction890, BOLD: AUGLY016-21; QM T250975.

\section{Description}

\section{Female}

COLOURATION. Gena without a pale spot; labrum mostly dark; scape colour in ventral half uniformly paler than flagellomeres; flagellomeres uniformly reddish-brown; tegula pale; wing veins uniformly black or brown, or with small lighter area proximally; anteromesoscutum dark with significant orange patches on posterolateral corners or dark with very slight orange patches on posterolateral corners; scutellar disk and metanotum dark; propodeum dark; hind coxa dark; fore femur pale yellow; mid femur pale yellow; hind femur pale yellow; fore tibia pale yellow; mid tibia pale yellow; hind tibia darkening posteriorly; hind basitarsus light brown; T1 dark reddish-brown; T2 sclerotised area dark reddish-brown; T2 lateral area dark extends past indentation, but then pale; T3 uniformly brown; T4+ reddish-brown.

Holotype BODY MEASUREMENTs. Body length $1.9 \mathrm{~mm}$; fore wing length $1.8 \mathrm{~mm}$; antennal length slightly longer than body length.

HEAD. Antennal flagellomere 14 length/width 1.14-1.28; antennal flagellomere 2 length/width 3.003.16; OOD/POD 2.17-2.20; IOD/POD 1.80-1.83.

Mesosoma. Anteromesoscutum sculpturing with shallow to deep punctures, space between punctures a mixture of smaller than diameter of punctures and of similar size, often smoother in posterior centre; scutellar disk sculpturing with shallow punctures scattered over most of area; seven pits in scutellar sulcus; propodeum with median carina absent and with punctured sculpturing in ' $\mathrm{v}$ ' shape in centre of propodeum, or with shallow sculpturing over most of propodeum other than smoother area in anterior centre.

WINGS. Pterostigma length $0.46 \mathrm{~mm}$; pterostigma width $0.15 \mathrm{~mm} ; \mathrm{r} 0.15 \mathrm{~mm} ; 2 \mathrm{RS} 0.12 \mathrm{~mm} ; 2 \mathrm{~m} 0.09 \mathrm{~mm}$; (RS+M)b $0.05 \mathrm{~mm}$.

Metasoma. T1 lateral edges parallel for anterior $1 / 2$ to $2 / 3$ of length, then narrowing posteriorly; T1 smooth and shiny; T1 length $0.32 \mathrm{~mm}$; T1 width at posterior edge $0.09 \mathrm{~mm}$; T2 an isosceles trapezoid, lateral edges straight; T2 smooth and shiny; T2 length $0.12 \mathrm{~mm}$; T2 width at posterior edge $0.3 \mathrm{~mm}$; ovipositor slightly protruding from end of metasoma.

\section{Male \\ Unknown.}

\section{Remarks}

Glyptapanteles lessardi sp. nov. constitutes BIN BOLD:AEI3823 and is 4.49\% (p-dist.) divergent from the closet BIN in the database (BOLD:AEI5416, an undescribed lineage from Australia, possibly part of G. wrightae sp. nov. (see details under G. wrightae sp. nov. Remarks section).

Using the BOLD Batch ID engine, the COI sequence of the holotype is $4.8 \%$ different from the most similar COI sequence from an Australian specimen (AUMIC084-18; Glyptapanteles wrightae sp. nov.).

\section{Distribution}

Currently known only from a single reserve in Cape York, the most northern region of QLD. 


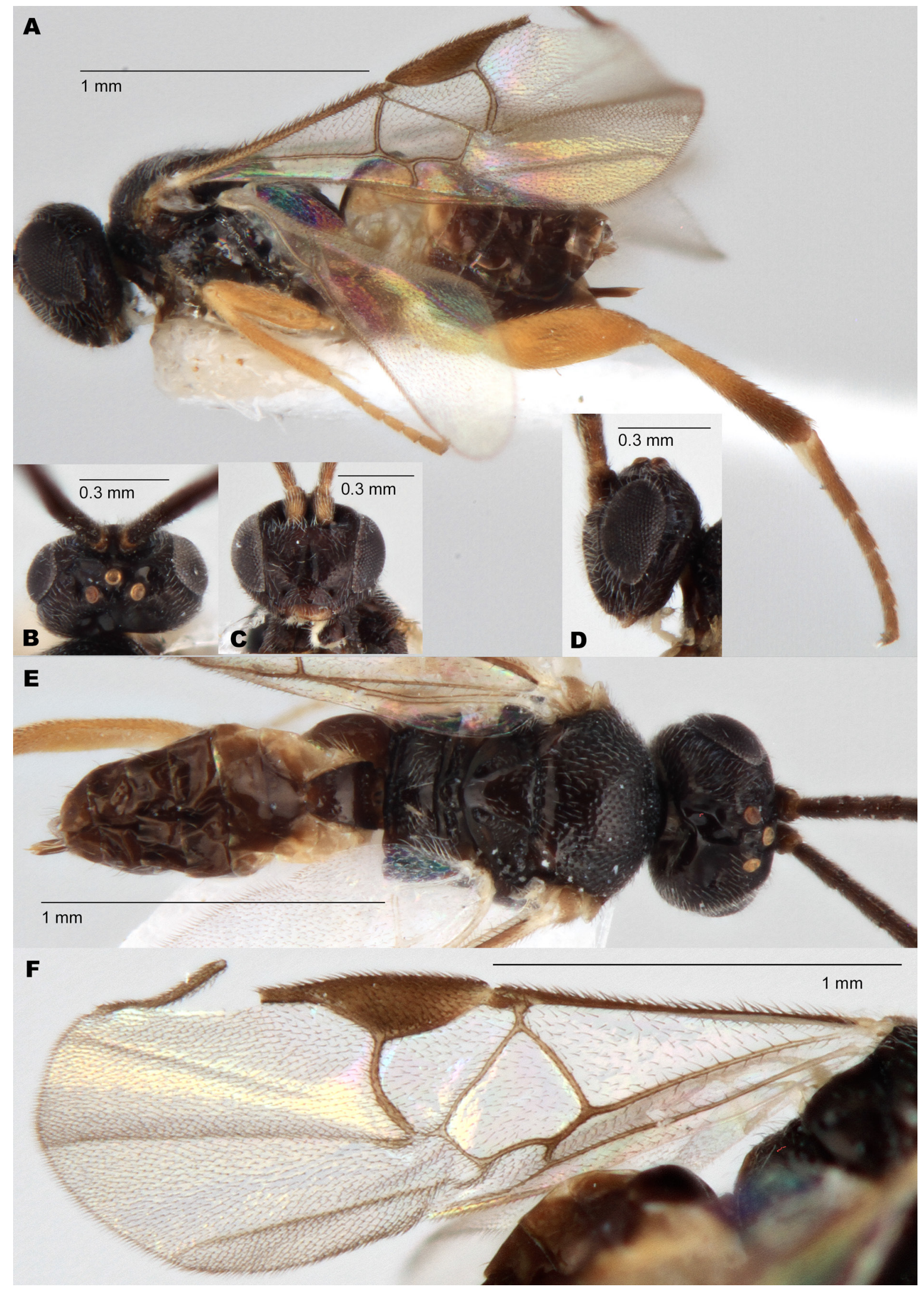

Fig. 43. Glyptapanteles lessardi Fagan-Jeffries, Bird \& Austin sp. nov., paratype, $ๆ$ (QM T250975). A. Lateral habitus. B. Dorsal head. C. Anterior head. D. Lateral head. E. Dorsal habitus. F. Fore wing. 


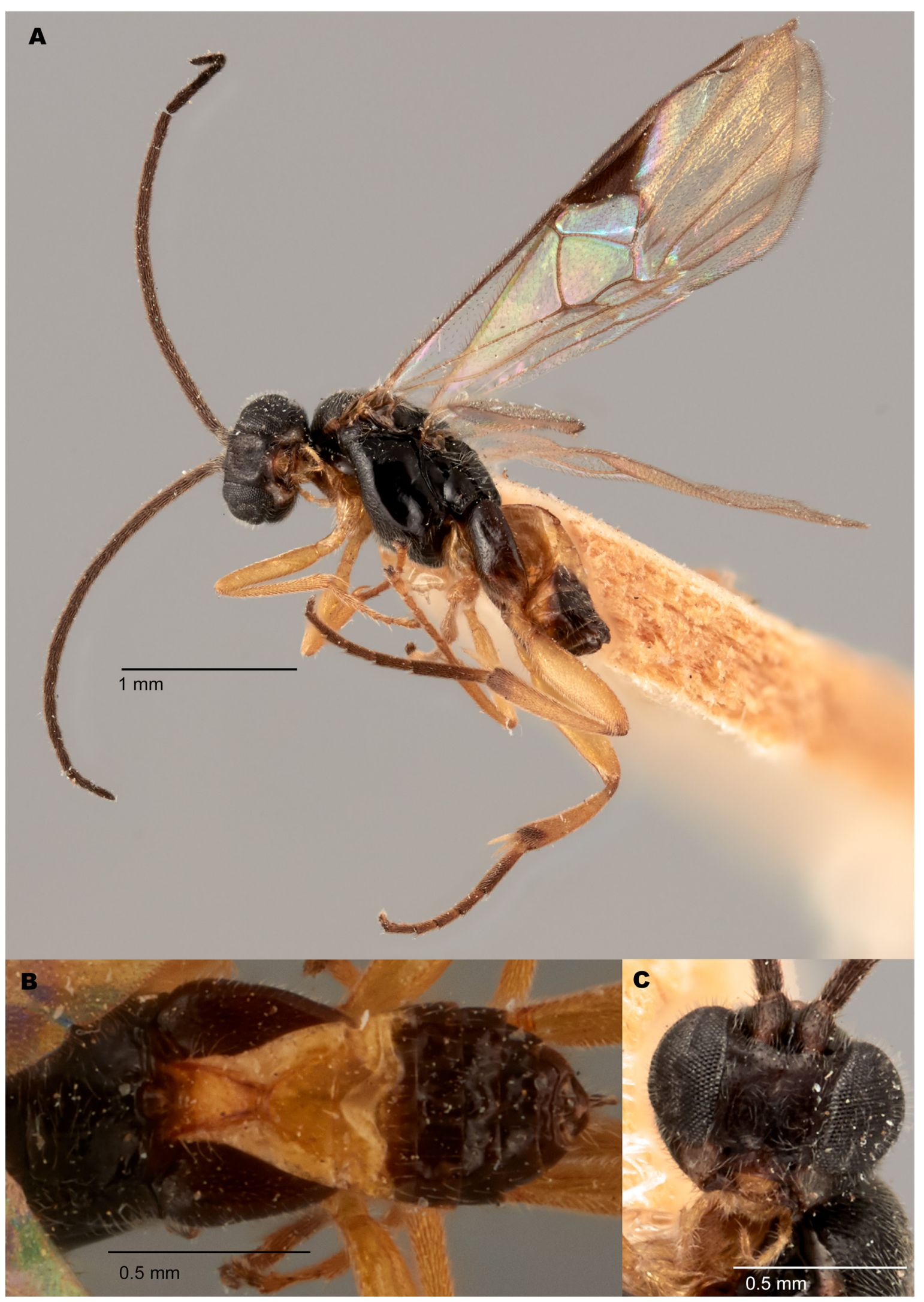

Fig. 44. Glyptapanteles mnesampela Austin, 2000 holotype, $q$ (ANIC 32-141445). A. Lateral habitus. B. Dorsal propodeum and metasoma. C. Anterior head. Images courtesy of O. Evangelista (ANIC). 
Glyptapanteles mnesampela Austin, 2000

Figs 6A, 44

\section{Diagnosis}

Glyptapanteles mnesampela is in the G. albigena species group and can be separated from the other members of the species group by having a pale T2, whilst all other described species with a pale spot on the gena have T2 dark in colouration.

\section{Material examined}

\section{Holotype}

AUSTRALIA • O; Australian Capital Territory, Lyneham Ridge; 30 Feb. 1993; ex Mnesampela privata; 22 Jun. 1993; ANIC 32-141445. (Only images examined).

\section{Remarks}

Reared from Mnesampela privata Guenée, 1858 (Geometridae).

\section{Distribution}

Known from Australia, currently only from the ACT.

Glyptapanteles mouldsi Fagan-Jeffries, Bird \& Austin sp. nov. urn:lsid:zoobank.org:act:03F851F6-74B6-4195-8254-B4BA372F1361

Figs $6 \mathrm{D}, 11 \mathrm{~A}-\mathrm{B}, 45$

\section{Diagnosis}

Glyptapanteles mouldsi sp. nov. is in the G. mouldsi species group and can be separated from the other two species in the group (G. rixi sp. nov. and G. dowtoni sp. nov.) by the metasomal sternites being all pale yellow other than the hypopygium, which is dark and T4-5 all pale/yellow. Glyptapanteles rixi sp. nov. and G. dowtoni sp. nov. have S5+ dark and T4-6 with at least some dark areas, or completely dark. The wingless sequences of the three species differ by at least 6 bp and the COI differs by at least $4.3 \%$.

\section{Etymology}

This species is named for Dr Max Moulds, who collected the type series, along with many other specimens, whilst kindly running a Malaise trap for this and other microgastrine projects at Kuranda, north QLD.

\section{Material examined}

\section{Holotype}

AUSTRALIA • क; Queensland, Kuranda; -16.8135, 145.6430586; 317 m a.s.1.; 12 Feb.-6 Apr. 2020; M.S. Moulds leg.; Malaise Trap EFJ2020MT36; Extraction1562, BOLD: AUGLY108-21; QM T250976.

\section{Paratypes}

AUSTRALIA - 1 क; same collection data as for holotype; Extraction1559, BOLD: AUGLY106-21; QM T250977 - 1 क; same collection data as for holotype; 16 Mar.-12 Apr. 2017; Extraction552, BOLD: AUMIC343-18; QM T250978 • 1 क; same collection data as for holotype; 18 Apr.-8 Jul. 2017; Extraction735, BOLD: AUMIC488-18; QM T250979.

\section{Description}

\section{Female}

Colouration. Gena without a pale spot; labrum mostly dark or reddish-brown; scape colour in ventral half either uniformly paler than flagellomeres, or the same colour or darker than flagellomeres; 


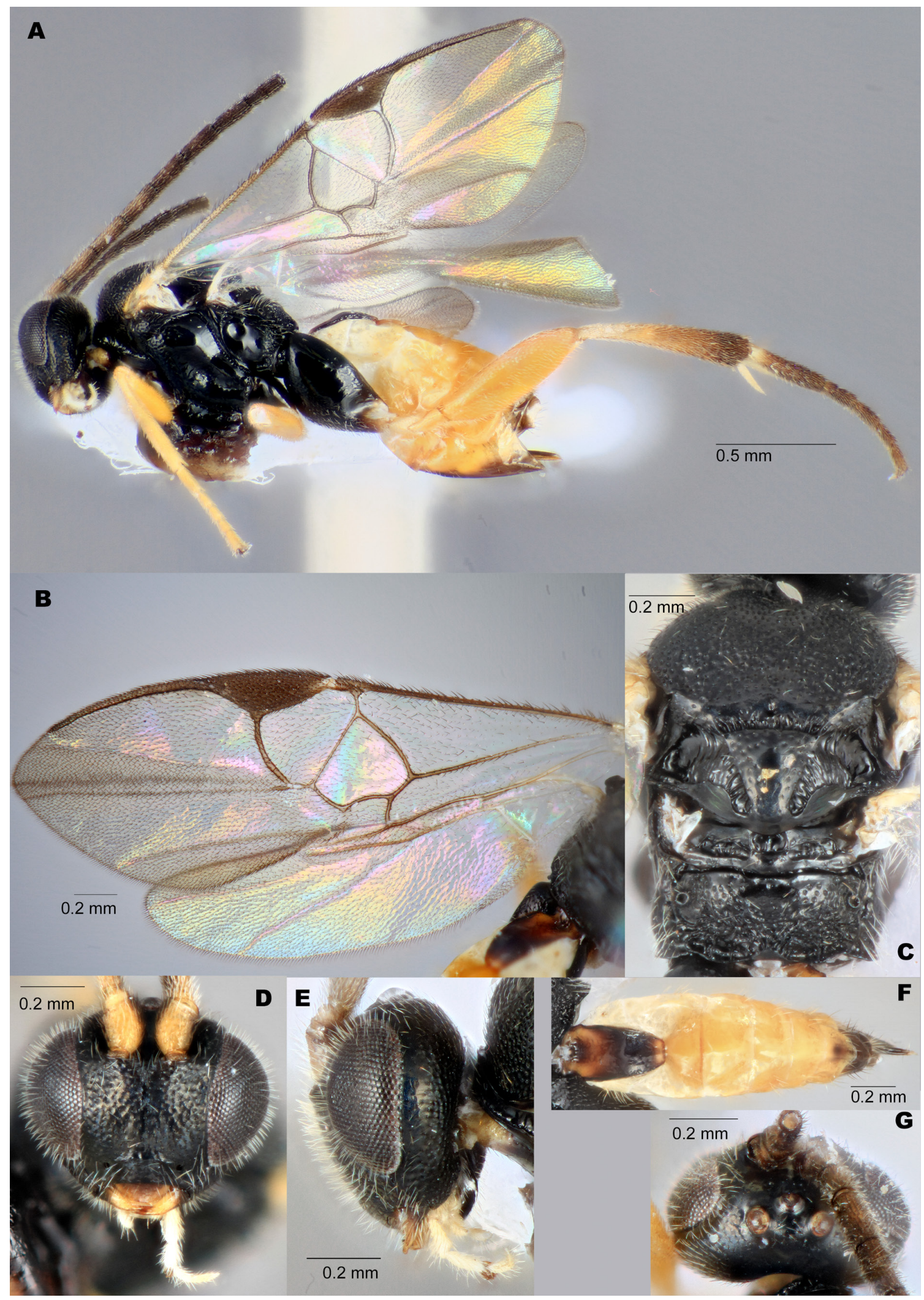

Fig. 45. Glyptapanteles mouldsi Fagan-Jeffries, Bird \& Austin sp. nov., paratypes, ૧. A-F. QM T250978. G. QM T250979. A. Lateral habitus. B. Fore wing. C. Dorsal mesosoma. D. Anterior head. E. Lateral head. F. Dorsal metasoma. G. Dorsal head. 
flagellomeres darkening distally; tegula pale; wing veins uniformly black or brown, or with small lighter area proximally; anteromesoscutum all dark; scutellar disk and metanotum dark; propodeum dark; fore coxa dark; mid coxa dark; hind coxa dark; fore femur pale yellow; mid femur pale yellow; hind femur orange to light brown; fore tibia pale yellow; mid tibia pale yellow; hind tibia darkening posteriorly; hind basitarsus dark reddish-brown; T1 dark or dark reddish-brown; T2 sclerotised area pale; T2 lateral area same colour as sclerotised area, or only slightly paler; T3-T5 pale, T6-7 darkening posteriorly.

Holotype Body MEASUREMENTs. Body length $2.3 \mathrm{~mm}$; fore wing length $2.2 \mathrm{~mm}$; antennal length slightly longer than body length.

HEAD. Antennal flagellomere 14 length/width 1.83-2.16; antennal flagellomere 2 length/width 1.773.00; OOD/POD 1.37-1.71; IOD/POD 1.57-1.75.

Mesosoma. Anteromesoscutum sculpturing with shallow to deep punctures, space between punctures a mixture of smaller than diameter of punctures and of similar size. Often smoother in posterior centre; scutellar disk sculpturing with deep or shallow, sparse, irregularly spaced punctures, more common on anterior lateral edges; 9-12 pits in scutellar sulcus; propodeum with median carina absent, strong punctures in anterior half, rugose sculpturing in posterior half.

WINGS. Pterostigma length $0.54 \mathrm{~mm}$; pterostigma width $0.18 \mathrm{~mm} ; \mathrm{r} 0.28 \mathrm{~mm} ; 2 \mathrm{RS} 0.12 \mathrm{~mm} ; 2 \mathrm{~m} 0.05 \mathrm{~mm}$; (RS+M)b $0.1 \mathrm{~mm}$.

Metasoma. T1 lateral edges parallel for anterior $1 / 4$ of length, then gently narrowing posteriorly, lateral edges often with slight curve or lateral edges parallel for anterior $3 / 4$ of length, then narrowing posteriorly; T1 smooth and shiny, sometimes with shallow sculpturing along lateral edges or some shallow scattered punctures on lateral edges; T1 length $0.3 \mathrm{~mm}$; T1 width at posterior edge $0.09 \mathrm{~mm}$; T2 an isosceles trapezoid, lateral edges straight; T2 smooth and shiny; T2 length $0.14 \mathrm{~mm}$; T2 width at posterior edge $0.21 \mathrm{~mm}$; ovipositor slightly protruding from end of metasoma.

\section{Male}

Unknown.

\section{Remarks}

Glyptapanteles mouldsi sp. nov. constitutes BIN: BOLD:ADL3640 and is 4.87\% (p-dist). divergent from the closet BIN in the database (BOLD:AEI8040, an undescribed lineage from Australia, with one specimen).

Using the BOLD Batch ID engine, the COI barcode of the holotype is $5.2 \%$ different from the most similar COI sequence from an Australian specimen (AUGLY141-21; an undescribed lineage, with a single specimen). All four of the type specimens were able to be sequenced for the wingless gene and share a unique barcode, which differs by a minimum of $5 \mathrm{bp}$ from all other species with available sequence data.

\section{Distribution}

This species is currently known from Kuranda in QLD.

Glyptapanteles niveus Fagan-Jeffries, Bird \& Austin sp. nov. urn:lsid:zoobank.org:act:DB934891-C1E7-4C64-97A8-0F1E5D31D6B1

Fig. 46

\section{Diagnosis}

Glyptapanteles niveus sp. nov. is in the G. niveus species group and can be differentiated from G. bradfordae sp. nov. by the presence of white distal flagellomeres (at least flagellomeres 10-11), a dark labrum and 
sparse punctures on the hind coxa ( $G$. bradfordae sp. nov. has the flagellomeres 10-11 dark, the labrum pale in colour and denser punctures on the hind coxa). Glyptapanteles niveus sp. nov. can be separated from G. cooperi sp. nov. by having a dark labrum (G. cooperi sp. nov. has the labrum pale in colour).

\section{Etymology}

The species epithet 'niveus' is a Latin adjective meaning 'snowy' and refers to the white flagellomeres and white fore coxa, mid coxa, hind- trochanter and anterior metasomal sternites.

\section{Material examined}

Holotype

AUSTRALIA • + ; Queensland, Kuranda; -16.8135, 145.6430586; 317 m a.s.1.; 12 Feb.-6 Apr. 2020; M.S. Moulds leg.; Malaise Trap EFJ2020MT36; Extraction1527, BOLD: AUGLY096-21; QM T250980.

\section{Description}

\section{Female}

COLOURATION. Gena without a pale spot; labrum mostly dark; scape colour in ventral half the same colour or darker than flagellomeres; proximal nine flagellomere segments dark, at least some distal segments (flagellomeres 10-11) white (final five segments missing from type so colour unknown); tegula dark; wing veins uniformly black or brown, or with small lighter area proximally; anteromesoscutum dark with significant orange patches on posterolateral corners; scutellar disk and metanotum dark; propodeum dark; fore coxa white; mid coxa white; hind coxa dark; mid femur pale yellow; hind femur pale yellow; fore tibia pale yellow; mid tibia pale yellow; hind tibia light brown; hind basitarsus light brown; T1 dark; T2 sclerotised area dark reddish-brown; T2 lateral area dark extends past indentation, but then pale; T3 uniformly brown; T4+ reddish-brown.

Holotype BODY MEASUREMENTs. Body length $2.1 \mathrm{~mm}$; fore wing length $1.8 \mathrm{~mm}$; antennal length slightly shorter than body length.

HEAD. Antennal flagellomere 2 length/width 4.33; antennal flagellomere 14 missing; OOD/POD $1.57-$ 1.83; IOD/POD 1.17.

Mesosoma. Anteromesoscutum sculpturing with very sparse, deep punctures; scutellar disk sculpturing with only very shallow punctures, smooth and shiny; seven pits in scutellar sulcus; propodeum with median carina present, in some parts difficult to differentiate from surrounding course rugosity.

WINGS. Pterostigma length $0.52 \mathrm{~mm}$; pterostigma width $0.14 \mathrm{~mm} ; \mathrm{r} 0.1 \mathrm{~mm} ; 2 \mathrm{RS} 0.11 \mathrm{~mm} ; 2 \mathrm{~m} 0.09 \mathrm{~mm}$; (RS+M)b $0.07 \mathrm{~mm}$.

Metasoma. T1 lateral edges parallel for entirety of length, posterior corners rounded at boundary with T2; T1 mostly smooth, some punctures in posterior half; T1 length $0.32 \mathrm{~mm}$; T1 width at posterior edge $0.15 \mathrm{~mm}$; T2 an isosceles trapezoid, lateral edges straight; T2 smooth, with some very shallow punctures in posterior half; T2 length $0.11 \mathrm{~mm}$; T2 width at posterior edge $0.28 \mathrm{~mm}$; ovipositor slightly protruding from end of metasoma.

Male

Unknown.

\section{Remarks}

Glyptapanteles niveus sp. nov. constitutes BIN BOLD:AEI1197 and is 3.55\% (p-dist.) divergent from the closet BIN in the database (BOLD:ADD5918, a BIN containing one specimen (GMNGU216-16) from Papua New Guinea). 


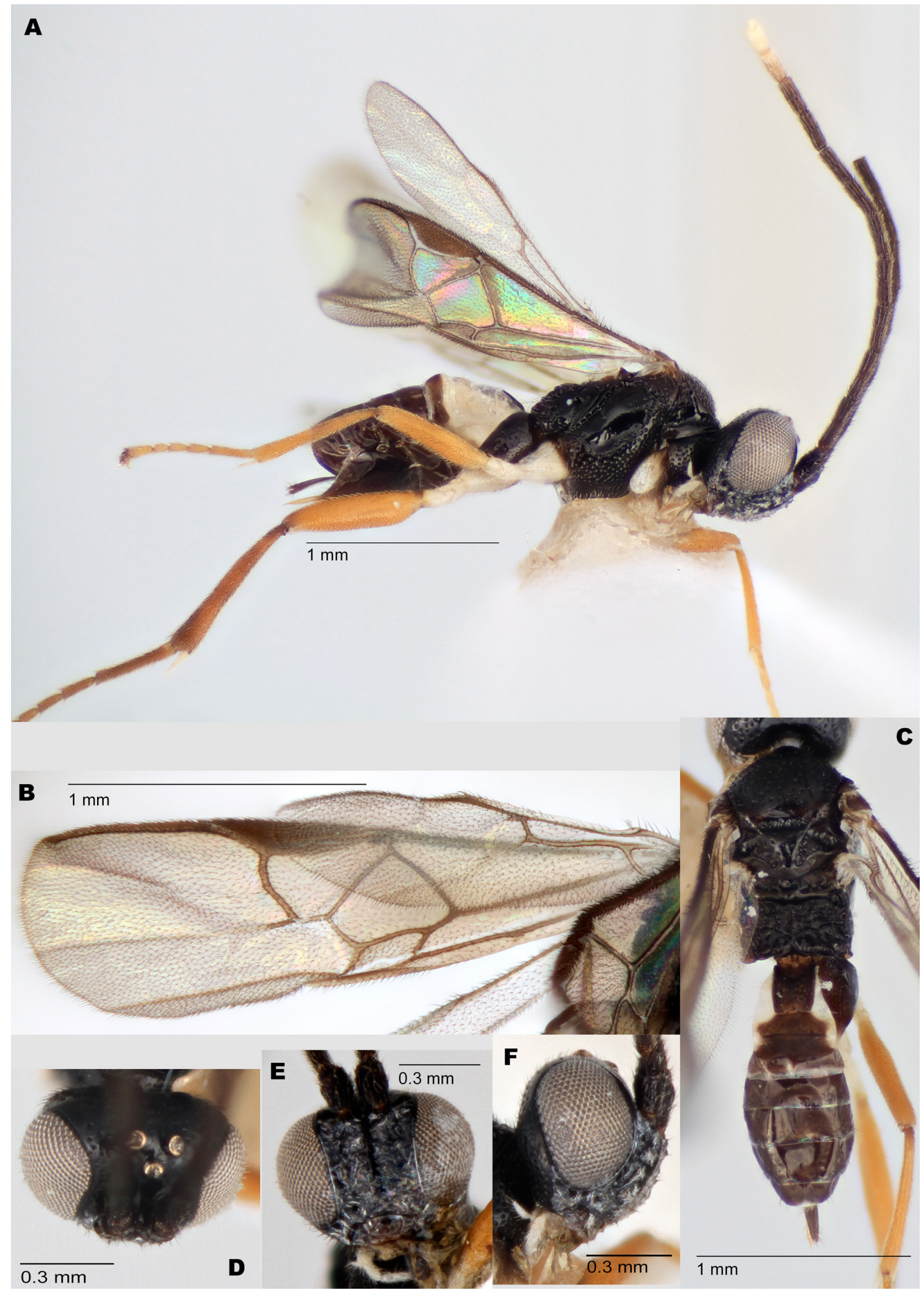

Fig. 46. Glyptapanteles niveus Fagan-Jeffries, Bird \& Austin sp. nov., holotype, $q$ (QM T250980). A. Lateral habitus. B. Fore wing. C. Dorsal habitus. D. Dorsal head. E. Anterior head. F. Lateral head. 
Using the BOLD Batch ID engine, the COI barcode of the holotype is $5.8 \%$ different from the most similar COI sequence from an Australian specimen (GMAQJ131-17; an undescribed lineage, with two specimens). The type specimen was sequenced for the wingless gene and the sequence differs by a minimum of $3 \mathrm{bp}$ from all other species with available sequence data.

\section{Distribution}

This species is only known from Kuranda in northern QLD.

Glyptapanteles operculinae (Fullaway, 1941)

Fig. 16D

\section{Diagnosis}

This species has a distinctive scutellum, with the lateral band extremely broad so that the lateral scutellum is compressed into a narrow crenulated sulcus. This, along with the T1 being extremely sharply narrowing posteriorly from a broad anterior width, means this species is very unlikely to be conspecific with any of the newly described Australian species.

\section{Material examined}

No specimens examined; illustrations and redescription in Austin \& Dangerfield (1992) used as reference (Fig. 16D).

\section{Distribution}

American Samoa, Western Samoa.

Glyptapanteles phytometrae (Wilkinson, 1928)

Fig. 47C, E

\section{Diagnosis}

Glyptapanteles phytometrae can be separated from many of the Australian species as follows: from the G. albigena species group by having the gena without a pale spot, from the G. mouldsi and G. eburneus species groups by having T1-2 dark and from the niveus species group by having a relatively smooth anteromesoscutum. The propodeum of $G$. phytometrae is mostly smooth with rugose sculpturing in the centre (Fig. 47C), which is distinct (at least on the available specimens) from any of the newly described species from Australia. Whilst propodeal sculpturing can vary slightly amongst individuals, we do not consider it likely that $G$. phytometrae is conspecific with any of the Australian species.

\section{Material examined}

Holotype

SAMOA • +; "Samoan Is. P.A. Buxton and G.H. Hopkins"; "Pres. by Imp. Bur. Ent. Brit. Mus. 1928318"; NHMUK: 3.c.1016 (only images examined).

\section{Remarks}

Known hosts in Samoa include Chrysodeixis eriosoma (Doubleday, 1843) and an unknown Plusiinae (Noctuidae).

Distribution (in the Australasian region)

Samoa, Fiji, Sumatra (Austin \& Dangerfield 1992). 


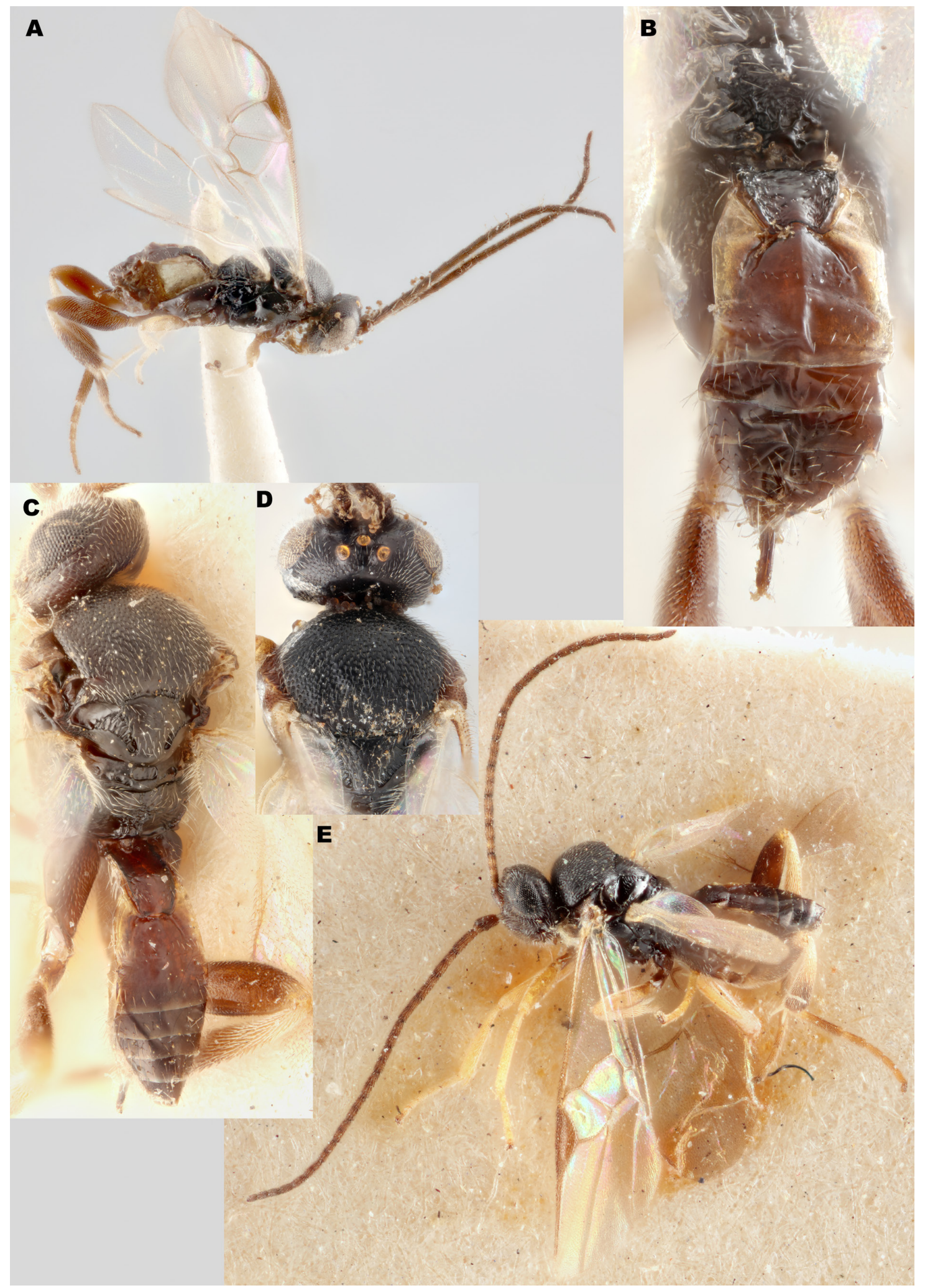

Fig. 47. A-B, D. Glyptapanteles taylori (Wilkinson, 1928), holotype, $q$ (NHMUK 3.c.1018). C, E. Glyptapanteles phytometrae (Wilkinson, 1928), holotype, o (NHMUK 3.c.1016). A. Lateral habitus. B. Dorsal propodeum and metasoma. C. Dorsal habitus. D. Dorsal mesosoma. E. Lateral habitus and fore wing. Images (C) The Trustees of the Natural History Museum, London (CC-BY 4.0). 
Glyptapanteles rixi Fagan-Jeffries, Bird \& Austin sp. nov. urn:lsid:zoobank.org:act:4BD5D589-8647-4922-8CC3-3457114C71AC Figs $6 \mathrm{C}, 10 \mathrm{~B}, 11 \mathrm{E}-\mathrm{F}, 48$

\section{Diagnosis}

Glyptapanteles rixi sp. nov. can be separated from G. mouldsi sp. nov. by having S5+ dark and T4-6 completely dark. Glyptapanteles mouldsi sp. nov. has the metasomal sternites all pale yellow other than the hypopygium, which is dark and T4-5 all pale/yellow. Glyptapanteles rixi sp. nov. can be separated from $G$. dowtoni sp. nov. by T4 being solidly dark (mostly pale in $G$. dowtoni sp. nov.) and $\mathrm{T} 1$ being orange to light brown (dark in G. dowtoni sp. nov.). The wingless sequences of the three species differ by at least $6 \mathrm{bp}$ and the COI differs by at least $4.3 \%$.

\section{Etymology}

This species is named for Dr Mike Rix, who collected the type series, along with many other specimens, whilst kindly running a Malaise trap for this and other microgastrine projects at Toohey State Forest in Brisbane.

\section{Material examined}

Holotype

AUSTRALIA - O; Queensland, Toohey State Forest; -27.5375, 153.057; 11 Dec. 2016-2 Jan. 2017; M. Rix leg.; Malaise trap; Extraction283, BOLD: AUMIC150-18; QM T250981.

\section{Paratypes}

AUSTRALIA • 1 क; same collection data as for holotype; Extraction282, BOLD: AUMIC149-18; QM T250982 - 1 त; same collection data as for holotype; Extraction1284, BOLD: AUGLY075-21; QM T250983 - 1 ; same collection data as for holotype; 29 Nov.-11 Dec. 2016; Extraction1285, BOLD: AUGLY076-21; QM T250984 - 1 क; same collection data as for holotype; Extraction1286, BOLD: AUGLY077-21; QM T250985.

\section{Description}

\section{Female}

Colouration. Gena without a pale spot; labrum mostly dark or reddish-brown; scape colour in ventral half uniformly paler than flagellomeres; flagellomeres darkening distally; tegula pale or light brown; wing veins uniformly black or brown, or with small lighter area proximally; anteromesoscutum all dark; scutellar disk and metanotum dark; propodeum dark; fore coxa dark; mid coxa dark; hind coxa dark; fore femur pale yellow; mid femur pale yellow; hind femur orange to light brown; fore tibia pale yellow; mid tibia pale yellow; hind tibia darkening posteriorly; hind basitarsus light brown; T1 orange to light brown; T2 sclerotised area dark reddish-brown, pale or orange to light brown; T2 lateral area same colour as sclerotised area, or only slightly paler or much paler; T3 mostly pale with darker patch in centre, mostly dark with paler lateral areas or uniformly brown; T4+ reddish-brown.

Holotype Body MEASUREMENTS. Body length $2.0 \mathrm{~mm}$; fore wing length $2.0 \mathrm{~mm}$; antennal length similar to body length.

HEAD. Antennal flagellomere 14 length/width 1.75; antennal flagellomere 2 length/width 2.89-4.00; OOD/POD 1.63-1.86; IOD/POD 1.38-2.00.

Mesosoma. Anteromesoscutum sculpturing with shallow to deep punctures, space between punctures a mixture of smaller than diameter of punctures and of similar size, often smoother in posterior centre; scutellar disk sculpturing with deep, sparse, irregularly spaced punctures, more common on anterior lateral edges or shallow punctures scattered over most of area; 8-9 pits in scutellar sulcus; propodeum with median carina absent, shallow rugose sculpturing with some punctures in anterior corners or median carina absent, smooth in centre and postero-lateral corners, rugose elsewhere. 


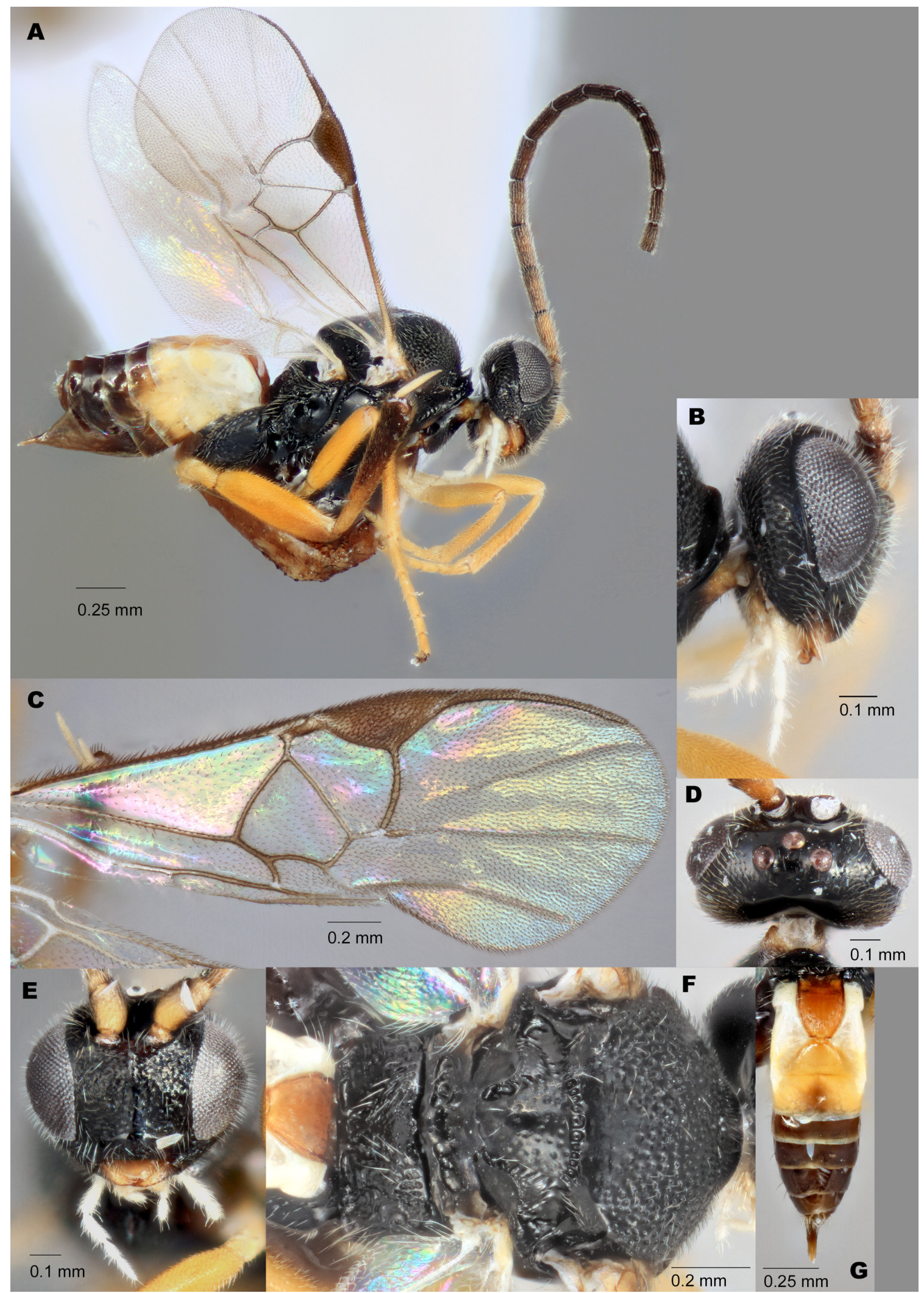

Fig. 48. Glyptapanteles rixi Fagan-Jeffries, Bird \& Austin sp. nov. A-C, E-G. Holotype, $q$ (QM T250981). D. Paratype, + (QM T250982). A. Lateral habitus. B. Lateral head. C. Fore wing. D. Dorsal head. E. Anterior head. F. Dorsal mesosoma. G. Dorsal metasoma. 
WINGS. Pterostigma length $0.52 \mathrm{~mm}$; pterostigma width $0.19 \mathrm{~mm}$; $\mathrm{r} 0.18 \mathrm{~mm} ; 2 \mathrm{RS} 0.13 \mathrm{~mm} ; 2 \mathrm{~m} 0.11 \mathrm{~mm}$; $(\mathrm{RS}+\mathrm{M}) \mathrm{b} 0.12 \mathrm{~mm}$.

Metasoma. T1 lateral edges parallel for anterior $1 / 2$ to $2 / 3$ of length, then narrowing posteriorly; T1 mostly smooth, some punctures in posterior half; T1 length $0.32 \mathrm{~mm}$; T1 width at posterior edge $0.08 \mathrm{~mm}$; T2 an isosceles trapezoid, lateral edges straight or with curved lateral and anterior edges, becoming archor semicircle-shaped; T2 smooth and shiny; T2 length $0.15 \mathrm{~mm}$; T2 width at posterior edge $0.32 \mathrm{~mm}$; ovipositor slightly protruding from end of metasoma.

\section{Male}

As female.

\section{Remarks}

Glyptapanteles rixi sp. nov. constitutes BIN: BOLD:ADL2963 and is 3.69\% (p-dist.) divergent from the closet BIN in the database (BOLD:AEI5416, an undescribed lineage from Australia, with two specimens).

Using the BOLD Batch ID engine, the COI barcode of the holotype is 3.5\% different from the most similar COI sequence from an Australian specimen (AUGLY139-21; an undescribed lineage). All five of the type specimens were able to be sequenced for the wingless gene and share a unique barcode, which differs by a minimum of $5 \mathrm{bp}$ from all other species with available sequence data.

\section{Distribution}

This species is currently known from Toohey State Forest in Brisbane, QLD.

Glyptapanteles rodriguezae Fagan-Jeffries, Bird \& Austin sp. nov. urn:lsid:zoobank.org:act:04BFEC74-5C1A-40F1-B10D-FAD918E18883

Fig. 49

\section{Diagnosis}

Glyptapanteles rodriguezae sp. nov. is in the G. arcanus species group and can be separated from the other members of the species group, other than from Glyptapanteles ruhri sp. nov., by the hind femur being pale in colouration and propodeum punctate with smooth areas between punctures and with littleto-no rugosity and $\mathrm{T} 1$ punctured in the posterior half. There was no external morphological character found which easily separates $G$. rodriguezae sp. nov. from G. ruhri sp. nov. and we do not provide a morphological diagnosis between these two species. The two species are sister lineages in the current phylogeny (Fig. 2), but Glyptapanteles ruhri sp. nov. is $6 \mathrm{bp}$ different in the wingless sequence from G. rodriguezae sp. nov. and $>6 \%$ divergent in the COI barcode and we therefore feel confident that they are different species.

\section{Etymology}

This species is named for Dr Juanita Rodriguez, who was part of the team who collected the type specimen and who has an endlessly enthusiastic and collaborative approach to hymenopteran research.

\section{Material examined}

\section{Holotype}

AUSTRALIA - O; Australian Capital Territory, CSIRO Black Mountain; -35.2744, 149.1115; 6 Dec. 2017-5 Jan. 2018; J. Rodriguez, C. Waichert, K.M. Bayless and T. Pleines leg.; Malaise 2, green dry wash; Extraction979, BOLD: AUGLY031-21; ANIC 32130332. 


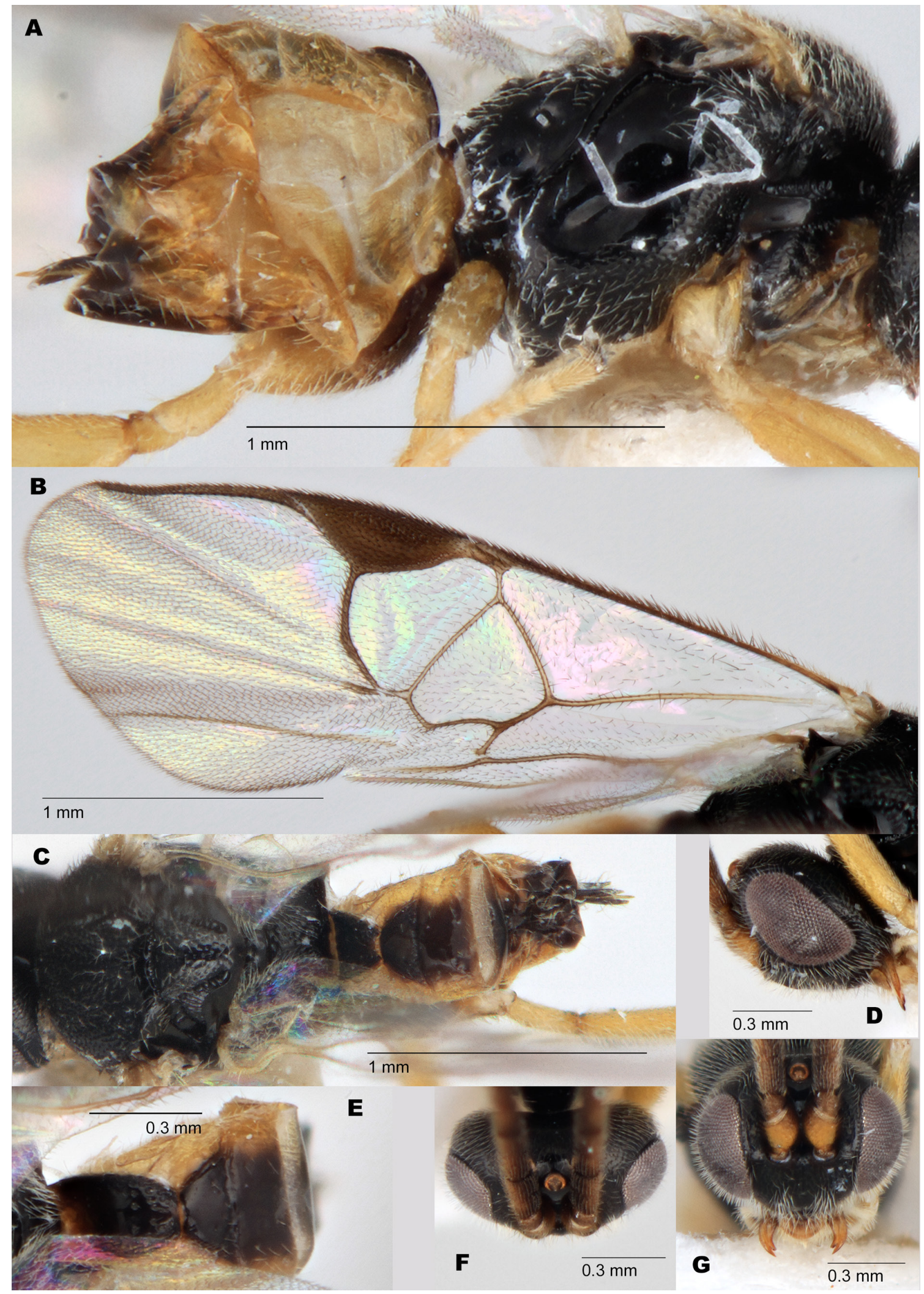

Fig. 49. Glyptapanteles rodriguezae Fagan-Jeffries, Bird \& Austin sp. nov., A, C, E. Paratype, $q$ (ANIC 32 130333). B, D, F-G. Holotype, $q$ (ANIC 32 130332). A. Lateral habitus. B. Fore wing. C. Dorsal habitus. D. Lateral head. E. Dorsal metasoma. F. Dorsal head. G. Anterior head. 
Paratype

AUSTRALIA - 1 \%; Australian Capital Territory, CSIRO Black Mountain; -35.268, 149.1066; 650 m a.s.l.; 11-25 Feb. 2020; K.M. Bayless leg.; Y-shaped wash, Malaise trap collapsed by flood; Extraction1138, BOLD: AUGLY062-21; ANIC 32130333.

\section{Description}

\section{Female}

Colouration. Gena without a pale spot; labrum reddish-brown; scape colour in ventral half uniformly paler than flagellomeres; flagellomeres all black/dark brown or darkening distally; tegula pale; wing veins uniformly black or brown, or with small lighter area proximally; anteromesoscutum all dark; scutellar disk and metanotum dark; propodeum dark; fore coxa pale yellow; mid coxa pale yellow; hind coxa dark; fore femur pale yellow; mid femur pale yellow; hind femur pale yellow; fore tibia pale yellow; mid tibia pale yellow; hind tibia darkening posteriorly; hind basitarsus light brown; T1 dark; T2 sclerotised area dark; T2 lateral area much paler; T3 mostly pale with darker patch in centre or mostly dark with paler lateral areas; T4-5 pale with dark patch in centre, then darkening posteriorly on T6-7.

Holotype BOdy MEASUREMENTs. Body length $2.4 \mathrm{~mm}$; fore wing length $2.4 \mathrm{~mm}$; antennal length slightly longer than body length.

HEAD. Antennal flagellomere 14 length/width 1.83-2.16; antennal flagellomere 2 length/width $2.87-$ 3.00; OOD/POD 1.75-2.17; IOD/POD 1.38-2.00.

Mesosoma. Anteromesoscutum sculpturing with shallow to deep punctures, space between punctures a mixture of smaller than diameter of punctures and of similar size, often smoother in posterior centre; scutellar disk sculpturing with shallow punctures scattered over most of area; 10-11 pits in scutellar sulcus; propodeum with median carina absent, propodeum punctate.

WINGS. Pterostigma length $0.63 \mathrm{~mm}$; pterostigma width $0.22 \mathrm{~mm} ; \mathrm{r} 0.23 \mathrm{~mm} ; 2 \mathrm{RS} 0.17 \mathrm{~mm} ; 2 \mathrm{~m} 0.12 \mathrm{~mm}$; $(\mathrm{RS}+\mathrm{M}) \mathrm{b} 0.07 \mathrm{~mm}$.

Metasoma. T1 lateral edges parallel for anterior $1 / 2$ to $2 / 3$ of length, then narrowing posteriorly; T1 mostly smooth, sometimes shiny, sometimes with punctures in posterior half and/or on lateral edges; T1 length $0.42 \mathrm{~mm}$; T1 width at posterior edge $0.12 \mathrm{~mm}$; T2 an isosceles trapezoid, lateral edges straight; T2 smooth and shiny or with some shallow indistinct sculpturing; T2 length $0.15 \mathrm{~mm}$; T2 width at posterior edge $0.26 \mathrm{~mm}$; ovipositor slightly protruding from end of metasoma.

\section{Male \\ Unknown.}

\section{Remarks}

Glyptapanteles rodriguezae sp. nov. constitutes BIN BOLD:AAU5026 and is $6.25 \%$ (p-dist.) divergent from the closet BIN in the database (BOLD:ADL3583; Glyptapanteles ruhri sp. nov.).

Using the BOLD Batch ID engine, the DNA barcode of the holotype is $6.3 \%$ different from the most similar sequence from an Australian specimen (AUMIC418-18; Glyptapanteles ruhri sp. nov.). Both type specimens were sequenced for the wingless gene, which is identical between them and differs by a minimum of $6 \mathrm{bp}$ from all other species with available sequence data. There is another specimen in the BOLD database, ASQAS187-11, which has an identical COI sequence, but the location data is listed only as Australia and there are no images available.

\section{Distribution}

This species is known from the ACT. 
Glyptapanteles ruhri Fagan-Jeffries, Bird \& Austin sp. nov. urn:1sid:zoobank.org:act:983624B0-EA3C-4AA3-B31B-54955164B7CB

Figs 13B, 50

\section{Diagnosis}

Glyptapanteles ruhri sp. nov. is in the G. arcanus species group and can be separated from the other members of the species group, other than from Glyptapanteles rodriguezae sp. nov., by having the hind femur pale in colouration, the propodeum punctate with smooth areas between punctures and with little-tono rugosity and $\mathrm{T} 1$ punctured in the posterior half. There was no external morphological character found which easily separates $G$. ruhri sp. nov. from $G$. rodriguezae sp. nov. and we do not provide a morphological diagnosis between these two species. The two species are sister lineages in the current phylogeny (Fig. 2), but Glyptapanteles ruhri sp. nov. is 6 bp different in the wingless sequence from G. rodriguezae sp. nov. and $>6 \%$ divergent in the COI barcode and we therefore feel confident that they are different species.

\section{Etymology}

Named for researcher Peter Rühr, who was part of the collection of the type specimen and contributed to the success of a field trip in northern QLD in 2019 that led to the collection of many important microgastrine specimens.

\section{Material examined}

\section{Holotype}

AUSTRALIA • 9 ; Queensland, Hallorans Hill Lookout, Atherton; -17.268, 145.4917268; $858 \mathrm{~m}$ a.s.1.; 21 Nov. 2019; E. Fagan-Jeffries, J.B. Dorey and P. Rühr leg.; sweeping vegetation; Extraction1198, BOLD: AUGLY071-21; QM T250986.

\section{Paratypes}

AUSTRALIA - Queensland • 1 کे; Woonoonooroon National Park scenic drive; -17.585, 145.7028957; 674 m a.s.l.; 21 Nov. 2019; E. Fagan-Jeffries, J.B. Dorey and P. Rühr leg.; sweeping vegetation; Extraction832, BOLD: AUGLY001-21; QM T250987 • 1 ô; same collection data as for preceding; Extraction844, BOLD: AUGLY002-21; QM T250988 • 1 q (ethanol); Lamington National Park; -28.21, 153.139; 474 m a.s.1.; 9-19 Apr. 2007; C. Lambkin and N. Starick leg.; IBISCA Plot \# IQ500-C rainforest Malaise trap; Extraction636, BOLD: AUMIC418-18; QM T208406 • 1 q; Lamington National Park; -28.148, 153.137; 267 m a.s.1.; 8-18 Jul. 2007; C. Lambkin and N. Starick leg.; IBISCA Plot \# IQ-300-A rainforest Malaise trap; Extraction639, BOLD: AUMIC420-18; QM T208407.

\section{Description}

\section{Female}

Colouration. Gena without a pale spot; labrum pale or reddish-brown; scape colour in ventral half uniformly paler than flagellomeres; flagellomeres all black/dark brown or uniformly reddish-brown; tegula pale; wing veins uniformly black or brown, or with small lighter area proximally; anteromesoscutum all dark or dark with significant orange patches on posterolateral corners; scutellar disk and metanotum dark; propodeum dark; fore coxa white; mid coxa white; hind coxa dark; fore femur pale yellow; mid femur pale yellow; hind femur pale yellow; fore tibia pale yellow; mid tibia pale yellow; hind tibia darkening posteriorly; hind basitarsus light brown; T1 dark; T2 sclerotised area dark or dark reddish-brown; T2 lateral area same colour as sclerotised area, or only slightly paler or dark extends past indentation, but then pale; T3 dark or uniformly brown; T4+ dark or reddish-brown.

Holotype Body MEASUREMENTs. Body length $1.9 \mathrm{~mm}$; fore wing length $2.1 \mathrm{~mm}$; antennal length slightly longer than body length. 


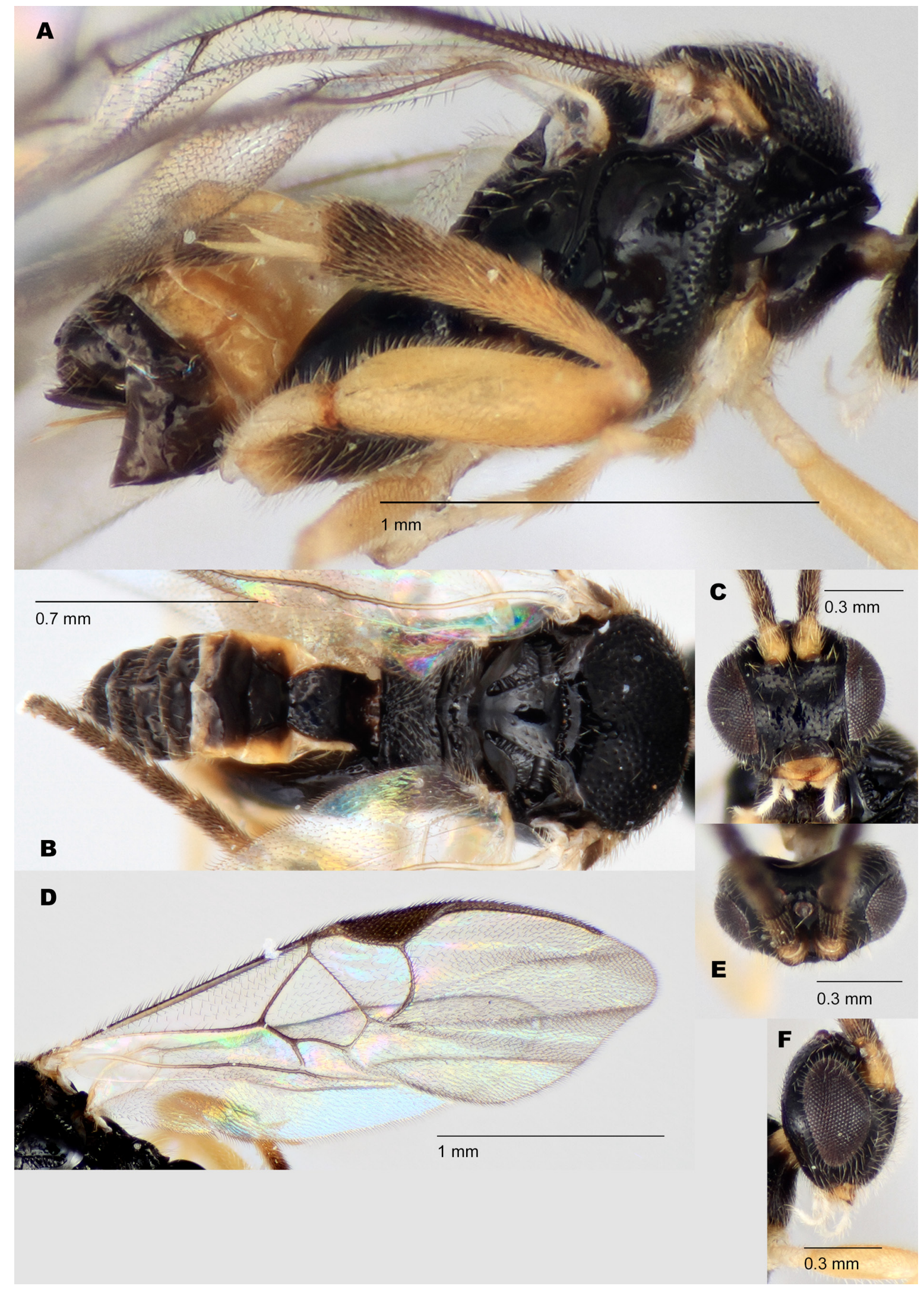

Fig. 50. Glyptapanteles ruhri Fagan-Jeffries, Bird \& Austin sp. nov., holotype, ㅇ (QM T250986). A. Lateral habitus. B. Dorsal habitus. C. Anterior head. D. Fore wing. E. Dorsal head. F. Lateral head. 
HeAD. Antennal flagellomere 14 length/width 1.57-2.00; antennal flagellomere 2 length/width 2.62 3.00; OOD/POD 1.57-1.71; IOD/POD 1.43.

Mesosoma. Anteromesoscutum sculpturing with very sparse, deep punctures; scutellar disk sculpturing with only very shallow punctures, smooth and shiny; 9-10 pits in scutellar sulcus; propodeum with median carina absent, irregular rugose sculpturing along centre line with punctures in anterior half of propodeum, postero-lateral areas smooth or median carina absent, propodeum mostly punctate with some irregular shallow rugosity in centre, postero-lateral corners smooth and shiny.

Wings. Pterostigma length $0.53 \mathrm{~mm}$; pterostigma width $0.16 \mathrm{~mm} ; \mathrm{r} 0.22 \mathrm{~mm} ; 2 \mathrm{RS} 0.14 \mathrm{~mm} ; 2 \mathrm{~m} 0.08 \mathrm{~mm}$; $(\mathrm{RS}+\mathrm{M}) \mathrm{b} 0.06 \mathrm{~mm}$.

Metasoma. T1 lateral edges parallel for anterior 3/4 of length, then narrowing posteriorly; T1 mostly smooth, some punctures in posterior half; T1 length $0.30 \mathrm{~mm}$; T1 width at posterior edge $0.14 \mathrm{~mm}$; T2 an isosceles trapezoid, lateral edges straight; T2 smooth and shiny, or smooth with some very shallow punctures in posterior half; T2 length $0.14 \mathrm{~mm}$; T2 width at posterior edge $0.2 \mathrm{~mm}$; ovipositor slightly protruding from end of metasoma.

\section{Male}

As female, 7-10 pits in scutellar sulcus.

\section{Remarks}

Glyptapanteles ruhri sp. nov. constitutes BIN BOLD:ADL3583 and is 6.25\% (p-dist.) divergent from the closet BIN in the database (BOLD:AAU5026; Glyptapanteles rodriguezae sp. nov.).

Using the BOLD Batch ID engine, the COI barcode of the holotype is $6.4 \%$ different from the most similar COI sequence from an Australian specimen (BOLD: ASQAS187-11, which was not included in the original study and AUGLY062-21; Glyptapanteles rodriguezae sp. nov.). All five type specimens were sequenced for the wingless gene, which is identical among the type specimens and differs by a minimum of $6 \mathrm{bp}$ from all other species with available sequence data.

\section{Distribution}

This species is known from a significant stretch of eastern QLD, from Atherton in northern QLD to Lamington National Park, south of Brisbane.

Glyptapanteles sanniopolus Fagan-Jeffries, Bird \& Austin sp. nov. urn:lsid:zoobank.org:act:B70D5575-E6F6-496F-8B47-453AD631CC2E

Figs $5 \mathrm{~B}, 51$

\section{Diagnosis}

Glyptapanteles sanniopolus sp. nov. is in the G. albigena species group and can be separated from most other members of the species group by having the gena with a large pale spot (Fig. 51G) rather than small (e.g., Fig. 40A) and from G. albigena sp. nov. by having the gena spot covering less than a third of the gena height and more rounded at the dorsal edge.

\section{Etymology}

The species epithet 'sanniopolus' is a combination of the Latin words 'sannio' ('one who makes faces') and 'polus' ('pole of the earth') and refers to the pale spot on the gena characteristic of this species and of the broader G. albigena species group. It is a noun in apposition. 


\section{Material examined}

Holotype

AUSTRALIA - $q$; New South Wales, Monga National Park near Penance Grove Walk; -35.597372, 149.912126; 9 Nov. 2019-16 Jan. 2020; K.M. Bayless and J.G. Lumbers leg.; Malaise trap over stream, trap destroyed by bushfire 30 Dec. 2019, partially dried out; Extraction1666, BOLD: AUGLY127-21; ANIC 32130370.

\section{Paratypes}

AUSTRALIA - New South Wales 1 \% ; same collection data as for preceding; Extraction1638, BOLD: AUGLY121-21; ANIC 32 130371. - Tasmania • 1 ○; Hobart, Kingston Beach; -42.986, 147.317; 14 18 Sep. 2010; B. Ward leg.; BIOUG00996-D09, BOLD: HYAT425-11; ANIC 32130372.

\section{Description}

\section{Female}

Colouration. Gena with a pale spot; labrum pale; scape colour in ventral half the same colour or darker than flagellomeres; flagellomeres all black/dark brown; tegula pale; wing veins uniformly black or brown, or with small lighter area proximally; anteromesoscutum all dark or dark with very slight orange patches on posterolateral corners; scutellar disk and metanotum dark; propodeum dark; fore coxa pale yellow; mid coxa pale yellow; hind coxa dark; fore femur pale yellow; mid femur pale yellow; hind femur pale yellow; fore tibia pale yellow or light brown; mid tibia light brown; hind tibia darkening posteriorly; hind basitarsus light brown; T1 dark; T2 sclerotised area dark or dark reddish-brown; T2 lateral area same colour as sclerotised area, or only slightly paler, or dark extends past indentation, but then pale; T3 dark or uniformly brown; T4+ dark or reddish-brown.

HoLOtyPe Body Measurements. Body length $2.4 \mathrm{~mm}$; fore wing length $2.6 \mathrm{~mm}$; antennal length slightly longer than body length.

HEAD. Antennal flagellomere 14 length/width 2.6; antennal flagellomere 2 length/width 4.33; OOD/ POD 1.86; IOD/POD 1.43.

Mesosoma. Anteromesoscutum sculpturing with shallow to deep punctures, space between punctures a mixture of smaller than diameter of punctures and of similar size. Often smoother in posterior centre; scutellar disk sculpturing with only very shallow punctures, smooth and shiny; nine pits in scutellar sulcus; propodeum with median carina present and complete, rest of propodeum strongly rugose.

WINGS. Pterostigma length $0.6 \mathrm{~mm}$; pterostigma width $0.23 \mathrm{~mm} ; \mathrm{r} 0.16 \mathrm{~mm} ; 2 \mathrm{RS} 0.15 \mathrm{~mm} ; 2 \mathrm{~m} 0.13 \mathrm{~mm}$; $(\mathrm{RS}+\mathrm{M}) \mathrm{b} 0.1 \mathrm{~mm}$.

METASOMA. T1 wedge-shaped, narrowing posteriorly for entirety of length, lateral edges straight (but not parallel); T1 smooth and shiny, some shallow scattered punctures on lateral edges and posterior half or smooth in anterior half, indistinct sculpturing in posterior half; T1 length $0.62 \mathrm{~mm}$; T1 width at posterior edge $0.1 \mathrm{~mm}$; T2 an isosceles trapezoid, lateral edges straight; T2 with some shallow indistinct sculpturing or smooth and shiny, some shallow punctures along posterior edge; T2 length $0.16 \mathrm{~mm}$; T2 width at posterior edge $0.27 \mathrm{~mm}$; ovipositor slightly protruding from end of metasoma.

\section{Male}

As female, 7-9 pits in scutellar sulcus. 


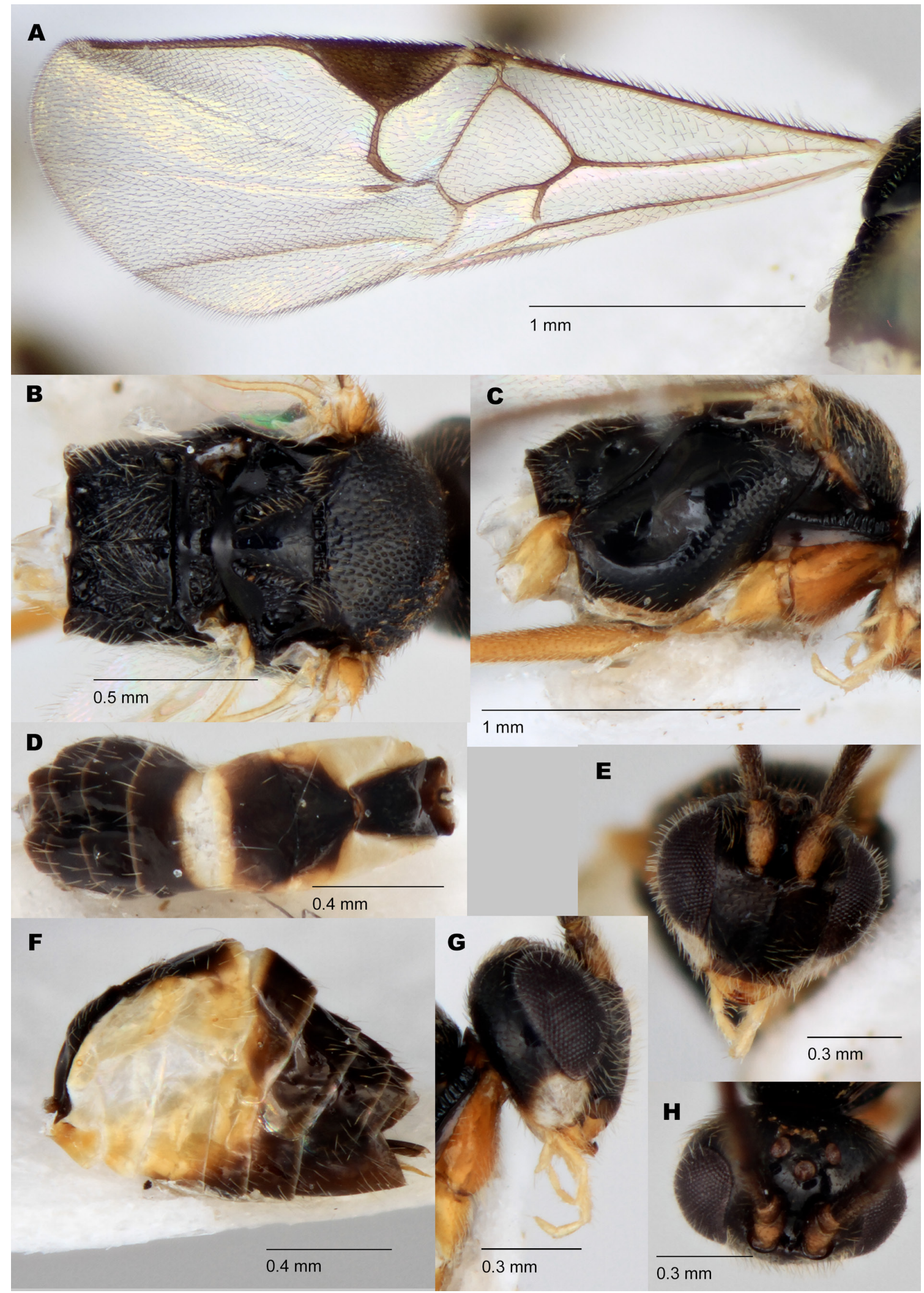

Fig. 51. Glyptapanteles sanniopolus Fagan-Jeffries, Bird \& Austin sp. nov., holotype, $q$ (ANIC 32 130370). A. Fore wing. B. Dorsal mesosoma. C. Lateral mesosoma. D. Dorsal metasoma. E. Anterior head. F. Lateral metasoma. G. Lateral head. H. Dorsal head. 


\section{Remarks}

Glyptapanteles sanniopolus sp. nov. constitutes BIN: BOLD:ABA6208 and is 5.48\% (p-dist.) divergent from the closet BIN in the database (BOLD:ADL3908; Glyptapanteles albigena sp. nov.).

Using the BOLD Batch ID engine, the COI barcode of the holotype is $5.6 \%$ different from the most similar COI sequence from an Australian specimen (AUMIC394-18; Glyptapanteles albigena sp. nov.). The two specimens able to be sequenced for the wingless gene share a unique barcode, which differs by a minimum of $3 \mathrm{bp}$ from all other species with available sequence data.

\section{Distribution}

This species is known from southern NSW and TAS.

Glyptapanteles taylori (Wilkinson, 1928)

Fig. $47 \mathrm{~A}-\mathrm{B}, \mathrm{D}$

\section{Diagnosis}

As Austin \& Dangerfield (1992) state that this species is unlikely to actually occur in the Australasian region and as we were unable to find morphological characters that definitively separate this species from all others in Australia, we do not diagnose it. There is a very low risk of this species being conspecific with any of the newly described species from Australia due to the likelihood that it does not actually occur in the region.

\section{Material examined}

\section{Holotype}

JAVA • +; “Java 1926 T.N.C. Taylor”; "Pres. by Imp. Bur. Ent. Brit. Mus. 1928-318”; NHMUK: 3.c. 1018 (only images examined).

\section{Distribution (in the Australasian region)}

Fiji (likely erroneous record), introduced.

Glyptapanteles vergrandiacus Fagan-Jeffries, Bird \& Austin sp. nov. urn:1sid:zoobank.org:act:C7A23777-0E24-4E31-80E4-2A398EC889F0

Fig. 52

\section{Diagnosis}

Glyptapanteles vergrandiacus sp. nov. is in the G. arcanus species group and can be separated from the other members of the species group (other than from G. arcanus sp. nov.) by having the propodeum with generally coarser sculpturing than most species, other than G. lambkinae sp. nov. and G. erucadesolator sp. nov. and with $\mathrm{T} 1$ less strongly sculptured than in G. lambkinae sp. nov. and G. erucadesolator sp. nov. It is noted, however, that all these species (along with G. goodwinnoakes sp. nov., which has T1 smooth) are morphologically very similar and identifications should be made with DNA barcodes. There was not a morphological character found to easily differentiate G. vergrandiacus sp. nov. from G. arcanus sp. nov., which are sister lineages in the current phylogeny (Fig. 2), but these two species differ in the wingless barcode by a single base pair and can also be separated by the COI barcodes, which have a $>3 \%$ divergence.

\section{Etymology}

The species epithet 'vergrandiacus' is a combination of the Latin words 'vergrandis', meaning 'little' and 'acus', meaning 'needle' and refers to the short ovipositor present on this species. It is a noun in apposition. 


\section{Material examined}

Holotype

AUSTRALIA - +; Queensland, Kuranda; -16.8135, 145.6430586; 317 m a.s.1.; 12 Feb.-6 Apr. 2020; M.S. Moulds leg.; Malaise Trap EFJ2020MT36; Extraction1548, BOLD: AUGLY102-21; QM T250989.

Paratypes

AUSTRALIA - Queensland - 1 ; ; same collection data as for holotype; Extraction1521, BOLD: AUGLY093-21; QM T250990 - 1 ; ; same collection data as for holotype; Extraction1525, BOLD: AUGLY095-21; QM T250991 - 1 q; same collection data as for holotype; Extraction1541, BOLD: AUGLY097-21; QM T250992 - 1 q; same collection data as for holotype; Extraction1555, BOLD: AUGLY105-21; QM T250993 - 1 +; same collection data as for holotype; Extraction1560, BOLD: AUGLY107-21; QM T250994 • 1 क; same collection data as for preceding; 16 Nov.-13 Dec. 2019; Extraction924, BOLD: AUGLY019-21; QM T250995 - 1 o ; same collection data as for holotype; 14 Dec. 2019 -6 Jan. 2020; Extraction1097, BOLD: AUGLY047-21; QM T250996 • 1 q (ethanol); same collection data as for holotype; 8 Jan.-11 Feb. 2020: Extraction1511, BOLD: AUGLY090-21; QM T250997 - 1 क ; same collection data as for holotype; Extraction1513, BOLD: AUGLY091-21; QM T250998 - 1 क (ethanol); same collection data as for holotype; 19 May-8 Jul. 2017; Extraction731, BOLD: AUMIC484-18; QM T250999.

\section{Description}

\section{Female}

Colouration. Gena without a pale spot; labrum reddish-brown; scape colour in ventral half uniformly paler than flagellomeres; flagellomeres uniformly reddish-brown or darkening distally; tegula dark; wing veins uniformly black or brown, or with small lighter area proximally; anteromesoscutum all dark; scutellar disk and metanotum dark; propodeum dark; fore coxa dark; mid coxa dark; hind coxa dark; fore femur pale yellow; mid femur pale yellow or dark proximally, lightening distally; hind femur dark; fore tibia pale yellow; mid tibia pale yellow; hind tibia darkening posteriorly; hind basitarsus light brown; T1 dark; T2 sclerotised area dark; T2 lateral area dark extends past indentation, but then pale; T3 mostly dark with paler lateral areas; T4+ dark.

Holotype Body MEASUREMENTs. Body length $2.2 \mathrm{~mm}$; fore wing length $2.2 \mathrm{~mm}$; antennal length similar to body length.

HEAD. Antennal flagellomere 14 length/width 1.62-2.16; antennal flagellomere 2 length/width 3.00 3.42; OOD/POD 1.71-2.17; IOD/POD 1.29-1.57.

Mesosoma. Anteromesoscutum sculpturing with shallow to deep punctures, space between punctures varying from smaller than diameter of punctures to larger than diameter of punctures, very sparse, deep punctures or very sparse, shallow to deep punctures; scutellar disk sculpturing with only very shallow punctures, smooth and shiny; 7-11 pits in scutellar sulcus; propodeum with median carina absent and propodeum strongly rugose.

WINGS. Pterostigma length $0.52 \mathrm{~mm}$; pterostigma width $0.16 \mathrm{~mm} ; \mathrm{r} 0.16 \mathrm{~mm} ; 2 \mathrm{RS} 0.13 \mathrm{~mm} ; 2 \mathrm{~m} 0.09 \mathrm{~mm}$; (RS+M)b $0.06 \mathrm{~mm}$.

Metasoma. T1 lateral edges parallel for anterior $3 / 4$ of length, then narrowing posteriorly or broadest at centre of length, narrowing both posteriorly and anteriorly from mid-point; T1 smooth in anterior half, indistinct sculpturing in posterior half; $\mathrm{T} 1$ length $0.35 \mathrm{~mm}$; T1 width at posterior edge $0.14 \mathrm{~mm}$; T2 an isosceles trapezoid, lateral edges straight; T2 smooth and shiny; T2 length $0.15 \mathrm{~mm}$; T2 width at posterior edge $0.27 \mathrm{~mm}$; ovipositor slightly protruding from end of metasoma. 


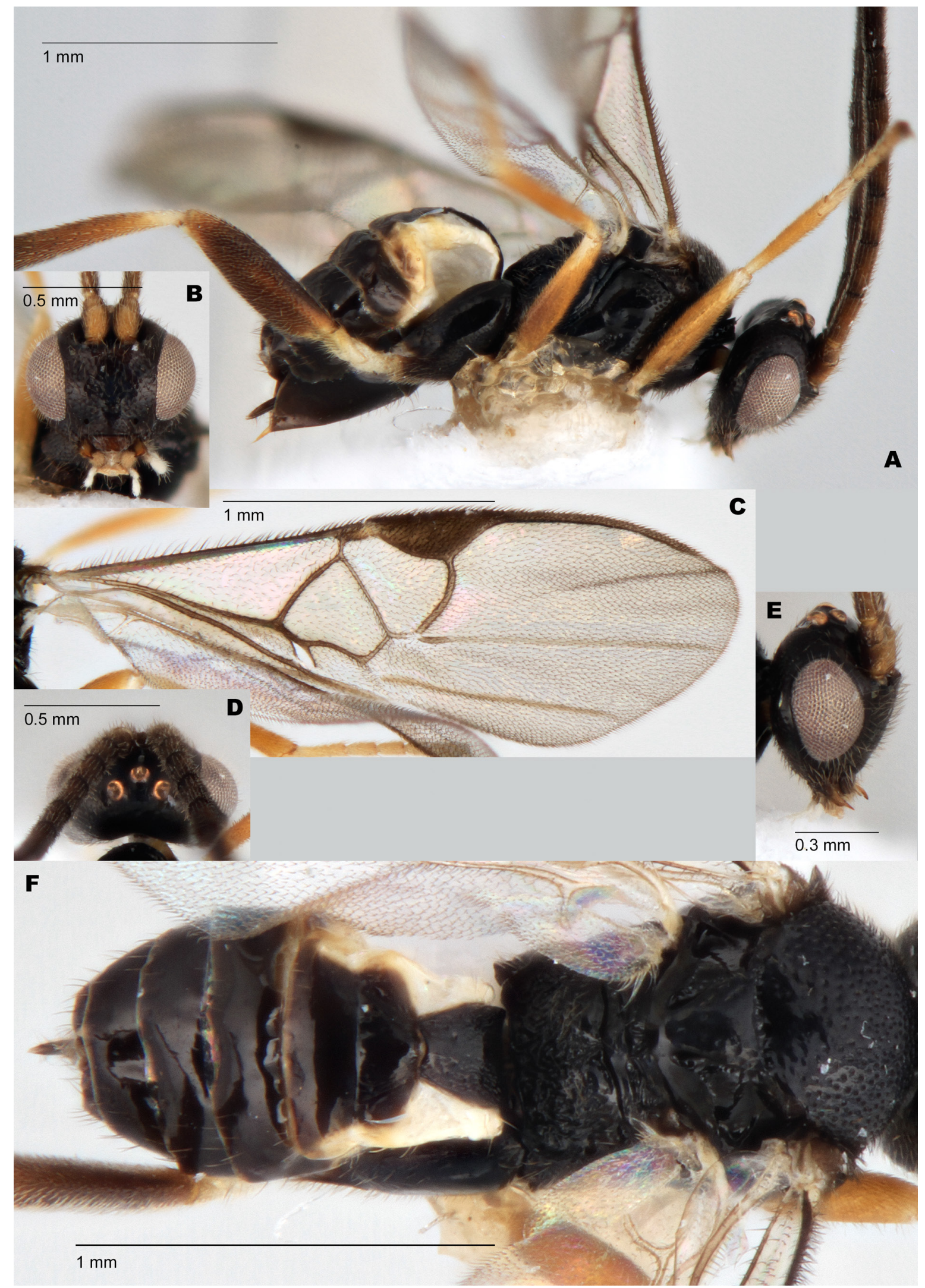

Fig. 52. Glyptapanteles vergrandiacus Fagan-Jeffries, Bird \& Austin sp. nov., paratype, $q$ (QMT250990). A. Lateral habitus. B. Anterior head. C. Fore wing. D. Dorsal head. E. Lateral head. F. Dorsal habitus. 


\section{Male \\ Unknown.}

\section{Remarks}

Glyptapanteles vergrandiacus sp. nov. constitutes BIN BOLD:ADL3012 and is 3.67\% (p-dist.) divergent from the closet BIN in the database (BOLD:ADL5262; Glyptapanteles arcanus sp. nov.).

Using the BOLD Batch ID engine, the COI sequence of the holotype is 3.6\% different from the most similar COI sequence from an Australian specimen (AUMIC423-18; Glyptapanteles arcanus sp. nov.). All of the type specimens were sequenced for the wingless gene, which is identical within the species and differs by a minimum of $1 \mathrm{bp}$ from all other species with available sequence data.

\section{Distribution}

This species is known only from Kuranda in northern QLD.

Glyptapanteles wrightae Fagan-Jeffries, Bird \& Austin sp. nov. urn:1sid:zoobank.org:act:569C2146-14FF-4453-BA4E-A6A8787A044A

Fig. 53

\section{Diagnosis}

Glyptapanteles wrightae sp. nov. can be separated from the other members of the arcanus species group as follows:

Glyptapanteles wrightae sp. nov. can be separated from G. rodriguezae sp. nov. and G. ruhri sp. nov. by $\mathrm{T} 1$ being smooth and shiny, not having punctures that cover at least a third of the area of the posterior half of the tergite.

Glyptapanteles wrightae sp. nov. can be separated from G. goodwinnoakes sp. nov., G. erucadesolator sp. nov., G. lambkinae sp. nov., G. arcanus sp. nov. and G. vergrandiacus sp. nov. by the propodeum being less coarsely and less consistently rugose sculptured across the anterior half of the propodeum. Glyptapanteles wrightae sp. nov. has the propodeum with strong punctures in the anterior half, the posterior half with shallow or strong rugose sculpturing.

Glyptapanteles wrightae sp. nov. can be separated from G. doreyi sp. nov. by the tegula being pale in colouration (the tegula in G. doreyi sp. nov. is generally dark in colouration), the hind femur mostly pale or light brown (mostly dark in $G$. doreyi sp. nov.) and the indentation in the centre of the mesopleuron being smooth, not strongly canaliculated as it is in G. doreyi sp. nov.

There was not a morphological character found that easily separates $G$. wrightae sp. nov. from G. lessardi sp. nov. and we do not diagnose these species morphologically. The wingless barcodes of the two species differ by 3 bp and the $C O I$ is greater than $4 \%$ divergent.

\section{Etymology}

Named for Susan Wright, who collected the holotype specimen. EPF-J would like to acknowledge Susan's support of visiting researchers at the QM and her continual generosity with her time and advice.

\section{Material examined}

\section{Holotype}

AUSTRALIA - ; Queensland, Samsonvale Cemetery, 8.5 km SSE of Dayboro; -27.2703, 152.856; 50 m a.s.1.; 6 Jan.-8 Feb. 2015; S. Wright leg.; Casuarina/open forest Malaise trap; Extraction198, BOLD: AUMIC084-18; QM T208400. 
Paratypes

AUSTRALIA - New South Wales • 1 गे; Royal National Park, near Waterfall Couranga Track near Hacking River; -34.1486, 151.0221; 40 m a.s.1.; 20 Jan.-6 Feb. 2020; K.M. Bayless and J.G. Lumbers leg.; 6 m Malaise trap over Waterfall Creek; Extraction857, BOLD: AUGLY128-21; ANIC 32130373 - 1 ô; same collection data as for preceding; Extraction863, BOLD: AUGLY010-21; ANIC 32130374.

\section{Description}

Female

COLOURATION. Gena without a pale spot; labrum reddish-brown; scape colour in ventral half uniformly paler than flagellomeres; flagellomeres darkening distally; tegula pale; wing veins uniformly black or brown, or with small lighter area proximally; anteromesoscutum all dark; scutellar disk and metanotum dark; propodeum dark; fore coxa white; mid coxa white; hind coxa dark; fore femur pale yellow; mid femur pale yellow; hind femur pale yellow; fore tibia pale yellow; mid tibia pale yellow; hind tibia darkening posteriorly; hind basitarsus light brown; T1 dark; T2 sclerotised area dark reddish-brown; T2 lateral area dark extends past indentation, but then pale; T3 mostly dark with paler lateral areas; T4+ reddish-brown.

Holotype BOdy MEASUREMENTs. Body length $1.8 \mathrm{~mm}$; fore wing length $1.8 \mathrm{~mm}$; antennal length slightly longer than body length.

HEAD. Antennal flagellomere 14 length/width 3.5; antennal flagellomere 2 length/width 2.71; OOD/ POD 1.83; IOD/POD 1.67.

Mesosoma. Anteromesoscutum sculpturing with deep punctures, space between punctures a mixture of smaller than diameter of punctures and of similar size; scutellar disk sculpturing with deep, sparse, irregularly spaced punctures, more common on anterior lateral edges or scutellar disk with shallow punctures scattered over most of area; eight pits in scutellar sulcus; propodeum with median carina absent, strong punctures in anterior half, rugose sculpturing in posterior half.

Wings. Pterostigma length $0.49 \mathrm{~mm}$; pterostigma width $0.16 \mathrm{~mm} ; \mathrm{r} 0.13 \mathrm{~mm}$; 2RS $0.14 \mathrm{~mm}$; $2 \mathrm{~m} 0.06 \mathrm{~mm}$; (RS+M)b $0.07 \mathrm{~mm}$.

Metasoma. T1 lateral edges parallel for anterior $1 / 2$ to $2 / 3$ of length, then narrowing posteriorly; T1 mostly smooth, some punctures in posterior half, smooth and shiny, sometimes with some shallow scattered punctures on lateral edges; T1 length $0.25 \mathrm{~mm}$; T1 width at posterior edge $0.08 \mathrm{~mm}$; T2 an isosceles trapezoid, lateral edges straight; T2 smooth and shiny; T2 length $0.14 \mathrm{~mm}$; T2 width at posterior edge $0.19 \mathrm{~mm}$; ovipositor slightly protruding from end of metasoma.

\section{Male}

As female.

\section{Remarks}

Glyptapanteles wrightae sp. nov. constitutes BIN BOLD:ADL3293 and is 1.76\% (p-dist.) divergent from the closet BIN in the database (BOLD:AEI5416; see information below about the specimens within this BIN).

Using the BOLD Batch ID engine, the COI sequence of the holotype is $1.7 \%$ different from the most similar COI sequence from an Australian specimen (AUGLY139-21, an undescribed lineage, with three sequences). This closely related lineage, (BOLD:AEI5416; also including BOLD AUGLY135-21 and AUGLY130-21) requires further study to determine whether it is within the species limits of G. wrightae sp. nov. or whether it represents a distinct species. 


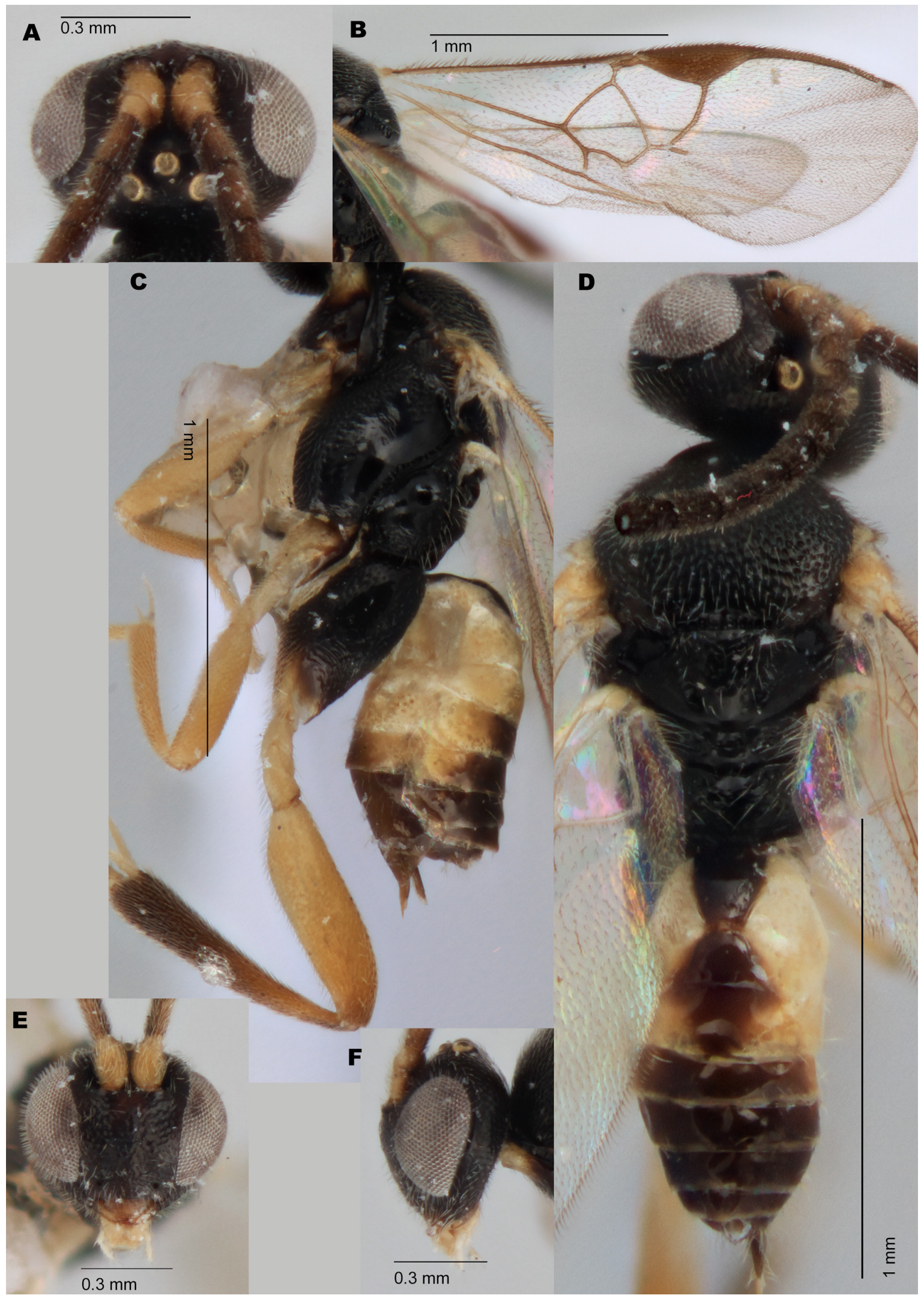

Fig. 53. Glyptapanteles wrightae Fagan-Jeffries, Bird \& Austin sp. nov., holotype, \& (QM T208400). A. Dorsal head. B. Fore wing. C. Lateral habitus. D. Dorsal habitus. E. Anterior head. F. Lateral head. 


\section{Distribution}

This species is currently known from southern QLD and north-eastern NSW.

\section{Discussion}

In this study we attempted to find a balance between producing robust species hypotheses which are accompanied by descriptions that are useful for end-user identifications and adhering to the Taxonomy Australia mission of documenting Australia's biodiversity at an accelerated pace. Whilst we believe the majority of our species descriptions will stand the test of time, this project makes only a tiny contribution to treating the huge number of undescribed species of Australian Microgastrinae. Whilst not worked on full-time, this study took 18 months of a postdoctoral researcher's time, with significant assistance from the co-authors. At this rate, it would take over 65 years to describe the remainder of the estimated 1300 undescribed microgastrine species in Australia. Changes to the traditional way of delimiting and describing species are needed to tackle these hyper-diverse, morphologically conserved taxa. Some authors have attempted to create this change by describing species based almost entirely on COI barcode BIN assignments on BOLD (Sharkey et al. 2021) with morphology barely used at all in descriptions or diagnoses, but this approach has received criticism (Ahrens et al. 2021; Meier et al. 2021). We have included morphology to a reasonably large extent in the diagnoses and key provided here, but have also described several species which are only diagnosable from their closest relative(s) by molecular information.

This approach is simply not tenable going forward, however, as even in this one genus and in the limited geographical scope of this study, there are nearly as many undescribed lineages as those that we were able to describe. Finding morphological characters to delimit a further 30 or so species, that are consistent and useful for non-experts, would not be an efficient or practical way to document the remaining Australian Glyptapanteles fauna that have DNA barcoded specimens available, let alone those that are yet to be collected and incorporated into a revision. A recent revision of Glyptapanteles from Costa Rica and Ecuador (Arias-Penna et al. 2019) described an impressive 136 species in a single study and provided a morphological key to species. Many of the characters used to separate species in Arias-Penna et al. (2019) are variable for the Australian species examined here and therefore not useful as diagnostic characters for all regions. We do not provide the answers to this dilemma here, but simply note that our approach, whilst limiting the application of morphological diagnoses, has not gone far enough to make the needed impact on increasing the rate at which new species of microgastrines are described.

As we relied on specimens that were preserved in ethanol and therefore suitable for DNA extraction, the sampling in this study is limited. Along with the lineages not described here, but recognised by sequenced specimens (Fig. 2), there is likely many more Glyptapanteles species yet to be collected, DNA barcoded and described in Australia. We make an assumption in this study that the species described here are endemic to Australia and Papua New Guinea and we do not morphologically diagnose them against Glyptapanteles species described from outside of the region. Whilst it is theoretically possible that there are either recent introductions or cosmopolitan species present in Australia, morphology in this group is not often reliable enough to make confident conclusions about species identification. We have ensured that none of our newly described species have COI barcodes $<2 \%$ different to anything available on BOLD (other than those mentioned in the remarks sections from Papua New Guinea) and consider that until a global effort to sequence historical type material is conducted, we are unable to categorically ensure that none of the new species have not already been described from another region.

A significant impediment to a modern revision of Australian parasitic wasps is the lack of host data for specimens that are of DNA-quality. Most specimens able to be DNA sequenced and incorporated into the current revision were collected by Malaise traps and sweep-netting and are therefore devoid of the 
critical biological information that can a) be used as a third piece of delimitation evidence alongside morphology and molecular information and b) allow the newly described species to be contextually placed in the environment. As such, we were not able to draw many conclusions about whether, for example, any of these new species are at risk of co-extinction due to parasitising endangered hosts, or whether any of these new species may be useful biological control agents for lepidopteran pests. A concerted effort to rear lepidopterans and their parasitoids is needed in Australia to allow more robust and more useful, taxonomic revisions to occur.

\section{Acknowledgements}

This study was supported by a Bush Blitz Tactical Taxonomy Grant (DNP-BSS-1920-002) and an Australian Biological Resources Study Research Grant (RG19-25). We thank the many collection managers who loaned and accessioned specimens used: Allison Brown and Jayme Sones (BIOUG), Olivia Evangelista de Souza (ANIC), Ben Parslow (SAMA), Derek Smith (AM), Nikolai Tatarnic (WAM) and Susan Wright and Karin Koch (QM). We also thank Olivia Evangelista de Souza for the images of the G. mnesampela holotype and Gavin Broad for the images of types in the NHMUK. We thank Peter Rühr and James Dorey for assistance in the field, along with many volunteers who ran Malaise traps around the country for extended periods during the course of this study: Karen Cullen (WA), Mark Dowton (NSW), Mark Harvey (WA), Mike Rix (QLD) Alison Goodwin and Roger Noakes (NSW), Kerri-Lee Harris and Paul Whitington (NSW), Max Moulds (QLD). We thank the Bush Blitz organisers and scientists for collecting microgastrines on Bush Blitz expeditions around Australia, which were used in this study. Finally we thank José Fernández-Triana and an anonymous reviewer for their invaluable comments which improved this manuscript. We thank the dedicated EJT editorial team for their detailed and conscientious handling of the manuscript. Specimens collected in QLD by or with J.B. Dorey were collected under permit PTU18-001277-1. This project represents a contribution to Taxonomy Australia (2021), a national initiative organised under the auspices of the Australian Academy of Science, which brings together the taxonomic community to develop approaches that will significantly increase the rate at which new species are discovered, resolved and named, with a view to completely documenting the Australian biota within a generation.

\section{References}

Ahrens D., Ahyong S.T., Ballerio A., Barclay M.V.L., Eberle J., Espeland M., Huber B.A., Mengual X., Pacheco T.L., Peters R.S., Rulik B., Vaz-de-Mello F., Wesener T. \& Krell F.-T. 2021. Is it time to describe new species without diagnoses? - A comment on Sharkey et al. (2021). Zootaxa 5027 (2): 151-159. https://doi.org/10.11646/zootaxa.5027.2.1

Arias-Penna D.C., Whitfield J.B., Janzen D.H., Hallwachs W., Dyer L.A., Smith M.A., Hebert P.D.N. \& Fernández-Triana J.L. 2019. A species-level taxonomic review and host associations of Glyptapanteles (Hymenoptera, Braconidae, Microgastrinae) with an emphasis on 136 new reared species from Costa Rica and Ecuador. ZooKeys 890: 1-685. https://doi.org/10.3897/zookeys.890.35786

Austin A.D. \& Dangerfield P.C. 1992. Synopsis of Australasian Microgastrinae (Hymenoptera: Braconidae), with a key to genera and description of new taxa. Invertebrate Systematics 6: 1-76. https://doi.org/10.1071/IT9920001

de Queiroz K. 1998. The general lineage concept of species, species criteria and the process of speciation. In: Endless Forms: Species and Speciation: 57-75. Oxford University Press, Oxford.

Eady R.D. 1968. Some illustrations of microsculpture in the Hymenoptera. Proceedings of the Royal Entomological Society of London. Series A, General Entomology 43: 66-72.

https://doi.org/10.1111/j.1365-3032.1968.tb01029.x 
Fagan-Jeffries E.P. \& Austin A.D. 2020. Synopsis of the parasitoid wasp genus Cotesia Cameron, 1891 (Hymenoptera: Braconidae: Microgastrinae) in Australia, with the description of seven new species. European Journal of Taxonomy 667: 1-70. https://doi.org/https://doi.org/10.5852/ejt.2020.667

Fagan-Jeffries E.P. \& Austin A.D. 2021. Four new species of parasitoid wasp (Hymenoptera: Braconidae) described through a citizen science partnership with schools in regional South Australia. Zootaxa 4949 (1): 79-101. https://doi.org/https://doi.org/10.11646/zootaxa.4949.1.4

Fagan-Jeffries E.P., Cooper S.J.B., Bertozzi T., Bradford T.M. \& Austin A.D. 2018. DNA barcoding of microgastrine parasitoid wasps (Hymenoptera: Braconidae) using high-throughput methods more than doubles the number of species known for Australia. Molecular Ecology Resources 18: 1132-1143. https://doi.org/10.1111/1755-0998.12904

Fagan-Jeffries E.P., Cooper S.J.B. \& Austin A.D. 2019. New species of Australian microgastrine parasitoid wasps (Hymenoptera: Braconidae: Microgastrinae) documented through the 'Bush Blitz' surveys of national reserves. Zootaxa 4560 (3): 401-440. https://doi.org/10.11646/zootaxa.4560.3.1

Fernández-Triana J., Shaw M.R., Boudreault C., Beaudin M. \& Broad G.R. 2020. Annotated and illustrated world checklist of Microgastrinae parasitoid wasps (Hymenoptera, Braconidae). ZooKeys 920: 1-1089. https://doi.org/10.3897/zookeys.920.39128

Fullaway D.T. 1957. Checklist of the Hymenoptera of Fiji. Proceedings of the Hawaiian Entomological Society 16: 269-280.

Gupta A., Venkatesan T. \& More R.P. 2016. Morphological and molecular characterization of reared parasitoid wasps of the genus Glyptapanteles Ashmead 1904 (Insecta: Hymenoptera: Braconidae: Microgastrinae) associated with Lepidoptera in India. PLoS ONE 11: e0150765.

https://doi.org/10.1371/journal.pone.0150765

Hoang D.T., Chernomor O., Von Haeseler A., Minh B.Q. \& Vinh L.S. 2018. UFBoot2: improving the ultrafast bootstrap approximation. Molecular Biology and Evolution 35: 518-522.

https://doi.org/10.1093/molbev/msx281

Kalyaanamoorthy S., Minh B.Q., Wong T.K.F., Von Haeseler A. \& Jermiin L.S. 2017. ModelFinder: fast model selection for accurate phylogenetic estimates. Nature Methods 14: 587-589.

https://doi.org/10.1038/nmeth.4285

Katoh K. \& Standley D.M. 2013. MAFFT multiple sequence alignment software version 7: improvements in performance and usability. Molecular Biology and Evolution 30: 772-780.

https://doi.org/10.1093/molbev/mst010

Katoh K., Misawa K., Kuma K.I. \& Miyata T. 2002. MAFFT: a novel method for rapid multiple sequence alignment based on fast Fourier transform. Nucleic Acids Research 30 (14): 3059-3066.

https://doi.org/10.1093/nar/gkf436

Mason W.R.M. 1981. The polyphyletic nature of Apanteles Foerster (Hymenopera: Braconidae): a phylogeny and reclassification of Microgastrinae. Memoirs of the Entomological Society of Canada 113: 1-147. https://doi.org/10.4039/entm113115fv

Meier R., Blaimer B., Buenaventura E., Hartop E., Rintelen T. von, Srivathsan A. \& Yeo D. 2021. A re-analysis of the data in Sharkey et al.'s (2021) minimalist revision reveals that BINs do not deserve names, but BOLD Systems needs a stronger commitment to open science. Cladistics 2021: 1-12. https://doi.org/10.1111/cla.12489

Nguyen L.T., Schmidt H.A., Von Haeseler A. \& Minh B.Q. 2015. IQ-TREE: a fast and effective stochastic algorithm for estimating maximum-likelihood phylogenies. Molecular Biology and Evolution 32 (1): 268-274. https://doi.org/10.1093/molbev/msu300 
Ratnasingham S. \& Hebert P.D.N. 2013. A DNA-based registry for all animal species: the Barcode Index Number (BIN) system. PLoS ONE 8: e66213. https://doi.org/10.1371/journal.pone.0066213

Rodriguez J.J., Fernández-Triana J.L., Smith M.A., Janzen D.H., Hallwachs W., Erwin T.L. \& Whitfield J.B.. 2013. Extrapolations from field studies and known faunas converge on dramatically increased estimates of global microgastrine parasitoid wasp species richness (Hymenoptera: Braconidae). Insect Conservation and Diversity 6: 530-536. https://doi.org/10.1111/icad.12003

Sharkey M.J., Janzen D.H., Hallwachs W., Chapman, E.G., Smith M.A., Dapkey T., Brown A., Ratnasingham S., Naik S., Manjunath R., Perez K., Milton M., Hebert P., Shaw S.R., Kittel R.N., Solis M.A., Metz M.A., Goldstein P.Z., Brown J.W., Quicke D.L.J., van Achterberg C., Brown B.V. \& Burns J.M. 2021. Minimalist revision and description of 403 new species in 11 subfamilies of Costa Rican braconid parasitoid wasps, including host records for 219 species. ZooKeys 2021: 1-665.

https://doi.org/10.3897/zookeys.1013.55600

Shenefelt R.D. 1972. Braconidae 4, Microgasterinae, Apanteles. In: van der Vecht J. \& Shenefelt R.D. (eds) Hymenopterorum Catalogus Part 7: 429-668. W. Junk. Gravenhage.

Taxonomy Decadal Plan Working Group 2018. Discovering Biodiversity: A Decadal Plan for Taxonomy and Biosystematics in Australia and New Zealand 2018-2028. Canberra and Wellington.

Available from https://www.science.org.au/ [accessed 4 Jan. 2022].

Wilkinson D.S. 1928. A revision of the Indo-Australian species of the genus Apanteles (Hym. Bracon.). Part II. Bulletin of Entomological Research 19: 109-146. https://doi.org/10.1017/S0007485300020393

Yeates D.K., Harvey M.S. \& Austin A.D. 2003. New estimates for terrestrial arthropod species-richness in Australia. Records of the South Australian Museum, Monograph Series 7: 231-241.

Manuscript received: 26 August 2021

Manuscript accepted: 9 November 2021

Published on: 8 February 2022

Topic editor: Nesrine Akkari

Section editor: Gavin Broad

Desk editor: Pepe Fernández

Printed versions of all papers are also deposited in the libraries of the institutes that are members of the EJT consortium: Muséum national d'histoire naturelle, Paris, France; Meise Botanic Garden, Belgium; Royal Museum for Central Africa, Tervuren, Belgium; Royal Belgian Institute of Natural Sciences, Brussels, Belgium; Natural History Museum of Denmark, Copenhagen, Denmark; Naturalis Biodiversity Center, Leiden, the Netherlands; Museo Nacional de Ciencias Naturales-CSIC, Madrid, Spain; Real Jardín Botánico de Madrid CSIC, Spain; Zoological Research Museum Alexander Koenig, Bonn, Germany; National Museum, Prague, Czech Republic.

\section{Supplementary files}

Supp. file 1. Specimen data for the phylogeny presented in Fig. 1, in the standard downloaded BOLD format (.xlsx). https://doi.org/10.5852/ejt.2022.792.1647.5927

Supp. file 2. Alignment file in PHYLIP format for the phylogeny presented in Fig. 1 (.txt). https://doi.org/10.5852/ejt.2022.792.1647.5929 
Supp. file 3. Specimen data for the phylogeny presented in Fig. 2, in the standard downloaded BOLD format (.xlsx). https://doi.org/10.5852/ejt.2022.792.1647.5931

Supp. file 4. Alignment file in PHYLIP format for the phylogeny presented in Fig. 2 (.txt). https://doi.org/10.5852/ejt.2022.792.1647.5933

Supp. file 5. Expanded phylogeny of the circular phylogeny presented in Fig. 1, support values are SH-aLRT support (\%) / ultrafast bootstrap support (\%) (.pdf). Due to the characteristics of this file, it is recommended to open it in a browser (i.e., Chrome, Safari) or with an application such as Preview Mac or similar. https://doi.org/10.5852/ejt.2022.792.1647.5935 